University of Warwick institutional repository: http://go.warwick.ac.uk/wrap

A Thesis Submitted for the Degree of PhD at the University of Warwick

http://go.warwick.ac.uk/wrap/77355

This thesis is made available online and is protected by original copyright.

Please scroll down to view the document itself.

Please refer to the repository record for this item for information to help you to cite it. Our policy information is available from the repository home page. 


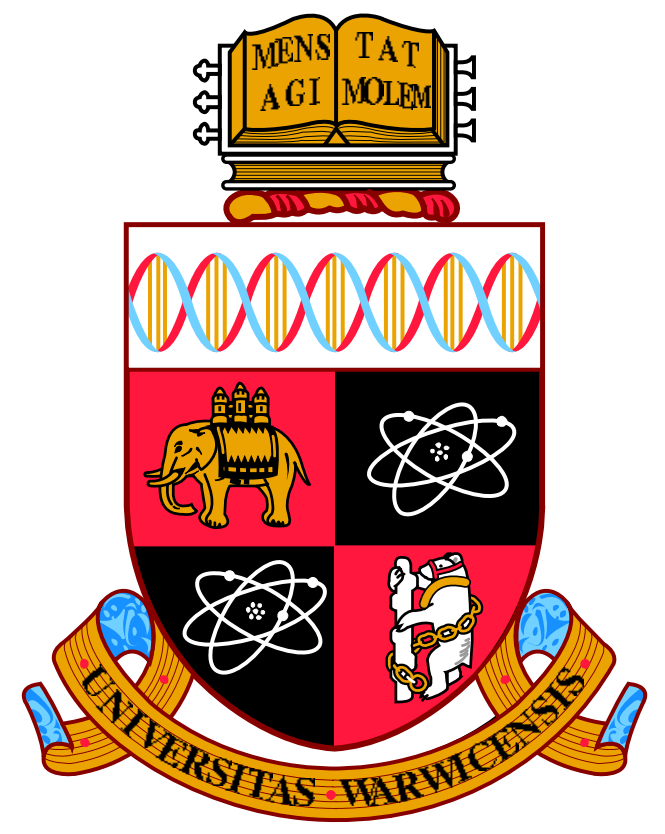

\title{
Topological Interactions in Ring Polymers
}

\author{
Davide Michieletto
}

A Thesis submitted to the

\section{University of Warwick}

for the degree of

Doctor of Philosophy in Physics and Complexity Science 


\section{Contents}

1 Introduction 1

2 Predicting the Behaviour of Rings in Solution 7

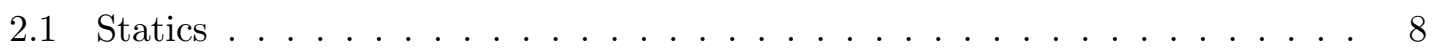

2.1.1 The Size of a Crumpled Coil . . . . . . . . . . . . 8

2.1.2 Contact Exponents for the Crumpled Globule . . . . . . . . . . 13

2.1 .3 The Structure Factor . . . . . . . . . . . . . . . . 15

2.2 Dynamics . . . . . . . . . . . . . . . . . . . . . 16

2.2.1 Diffusion Coefficient and Relaxation Time . . . . . . . . . 16

2.2 .2 How Rings Relax Stress . . . . . . . . . . . . . . . . . 19

2.2.3 Inter-Coil Correlations Probed by Dynamic Scattering . . . . . . . 20

3 Molecular Dynamics Models 23

3.1 Molecular Dynamics Scheme . . . . . . . . . . . . . . . . 24

3.1.1 Non-Bonded Potentials . . . . . . . . . . . . . . . . . 25

$3.1 .2 \quad$ Bonded Potentials . . . . . . . . . . . . . . . . 26

3.1 .3 Brownian Dynamics . . . . . . . . . . . . . . . 27

3.2 Modelling . . . . . . . . . . . . . . . . . . . . . . 30

3.2.1 Modelling (Knotted) Ring Polymers . . . . . . . . . . . . . . . 31

3.2 .2 Modelling a Physical Gel . . . . . . . . . . . . . 35

4 Threading Rings 38

4.1 Threading of Rings in a Gel . . . . . . . . . . . . . . . . . 39

4.1.1 Detecting Threadings between Rings . . . . . . . . . . . . . . 41

4.1.2 Extensive Threading Leads to Extensive Correlations . . . . . . . . 45

4.1.3 The Emergence of a Spanning Network of Inter-Threaded Chains . . 50

4.2 Threading of Rings in Dense Solutions . . . . . . . . . . . . . 52

4.2.1 Overlapping Crumpled Globules . . . . . . . . . . . 52

4.2.2 The Slow Exchange Dynamics of Rings . . . . . . . . . . . . 56

4.2.3 Inducing a Topological Glass by Randomly Pinning Rings . . . . . . 58

4.3 Conclusions . . . . . . . . . . . . . . . . 66 66 
5 A Bio-Physical Model for the Kinetoplast DNA 69

5.1 KDNA as an Ensemble of Diffusing Phantom Loops . . . . . . . . . . . . 73

5.2 Probing the Network Topology . . . . . . . . . . . . . . 755

5.3 An in silico Digestion . . . . . . . . . . . . . . . . . . . . . . 79

5.4 A Linked Network of Rings at the Percolating Point . . . . . . . . . . . 83

6 The Role of Topology in DNA Gel Electrophoresis 8

6.1 Gel Electrophoresis of DNA Rings and Strands . . . . . . . . . . . . . 89

6.1.1 Getting More from Pushing Less . . . . . . . . . . . . . . . . 90

6.1.2 Non-Equilibrium Response Theory . . . . . . . . . . . . . . . 92

6.1.3 Topology can Sense Disorder . . . . . . . . . . . . . . . 95

6.2 Gel Electrophoresis of DNA Knots . . . . . . . . . . . . . . . 95

6.2.1 Non-monotonic Speed of DNA Knots in Gel . . . . . . . . . . . . . 97

6.2.2 Entanglement with Dangling Ends . . . . . . . . . . . . . 999

6.2.3 An Equivalent Random Walk Description . . . . . . . . . . . . 103

6.3 Conclusions . . . . . . . . . . . . . . . . . . . . . 105

7 Conclusions 108

\begin{tabular}{ll} 
Appendices & $\mathbf{1 1 0}$ \\
\hline
\end{tabular}

A Identifying Knots

B Self-Threading of Rings in Dilute Solutions

C The Replication of KDNA

D Non-equilibrium form of Differential Mobility 136

E Gel Electrophoresis of DNA Knots

F Threading Identification Algorithm 144 


\section{Ringraziamenti Acknowledgements}

Vorrei ringraziare Stefania, anche se mai abbastanza, per tutti i sacrifici e le difficoltà che ha condiviso con me in questi quattro anni: ogni piccolo o grande momento di tristezza è servito a renderci ancor più risoluti ed è stato ricompensato con soddisfazioni ugualmente grandi. Grazie per i momenti felici, per aver detto sì e per i "com'è andata" a fine giornata: Spero di sentirli ogni giorno per molti anni ancora.

Vorrei ringraziare la mia famiglia: i miei genitori Elena e Daniele, nonni Berto, Anna e Palmira, zii Dante, Wally, Bruno e Alfreda, cugini Roberto, Barbara e Luca; ed anche Sonia, Domenico, Andrea e Bruna. Non c'è riposo più sereno che nel calore di chi ti vuole bene e ti fa sentire a casa, e non c'è persona più ingrata di colui che non lo apprezza.

I would like to thank the many old friends with whom we have been sharing experiences and emotions during the past ten years: Anna and Riccardo for always being in each-other thoughts even though being miles apart, Elisa for being a sweet friend, Anna and Stefano for their inspiring dedication to each-other, Alessio for his uniqueness, Bisi, Sara, Cece, Cicci, Zakk and Tommy for their precious friendship. We feel honoured to be part of such strong group of brilliant persons that has been scattered across Europe and nonetheless managed to remain whole.

I would like to thank the new friends we made in England: Peter and Jen for their kindness and welcoming hearts, Dario and Tom for their (too) many talents, Ellen for her teasing and Quentin for having patiently introduced us to life in England. Thanks also to Dayal, Simone and Marco: the friends we would turn to when felt like missing home.

Finally, I would like to thank who supervised me during these years and to whom I could turn to when in need of sharing ideas and seeking inspiration: Matthew, Gareth, Enzo and Davide. You have been not only an essential part of my academic life, but each one of you has also taught me something that will make me a better man, and for that I will be forever grateful. 


\section{Declaration}

This Thesis is submitted to the University of Warwick in support of my application for the degree of Doctor of Philosophy. It has been composed by myself and has not been submitted in any previous application for any degree.

The work presented (including data generated and data analysis) was carried by the author.

Parts of this Thesis have been published by the author:

(1) D. Michieletto, D. Marenduzzo, E. Orlandini, G. P. Alexander, M. S. Turner, Threading Dynamics of Ring Polymers in a Gel, ACS Macro Lett., 3, 255-259 (2014)

(2) D. Michieletto, D. Marenduzzo, E. Orlandini, G. P. Alexander, M. S. Turner, Dynamics of Self-Threading Polymers in a Gel, Soft Matter, 10, 5936$5944(2014)$

(3) D. Michieletto, E. Orlandini, M. S. Turner, Rings in Random Environments: Sensing Disorder Through Topology, Soft Matter, 11, 1100-1106 (2015)

(4) D. Michieletto, D. Marenduzzo, E. Orlandini, Is the Kinetoplast DNA a Percolating Network of Linked Rings at its Critical Point?, Phys. Biol., 12, 036001 (2015)

(5) D. Michieletto, D. Marenduzzo, M. S. Turner, Topology Regulation during Replication of the Kinetoplast DNA, sub judice (arXiv:1408.4237)

(6) D. Michieletto, D. Marenduzzo, E. Orlandini, Topological Patterns in Twodimensional Gel Electrophoresis of DNA Knots, Proc. Natl. Acad. Sci. USA, 112(40), E5471-E5477 (2015) 
Below, I will describe (i) how the material published in the aforementioned papers is reported and distributed in the Thesis Chapters and (ii) the respective contribution of the authors to the papers:

The material published in paper (1) is described in Chapter 4 of the Thesis. The material of paper (2), although similar in spirit to paper (1), has been described in Appendix B of this Thesis. This choice reflects the different numerical methods used to simulate the systems considered. The goals and physical ideas behind these papers (and of Chapter 4 and Appendix B), have been inspired by my supervisor Prof. Matthew Turner. At the start of my PhD I did not possess the numerical expertise needed to set up these complex simulations. I therefore sought the expertise of my external supervisor Dr. Davide Marenduzzo and of my former MSc supervisor Prof. Enzo Orlandini, who suggested the right numerical tools to use. Throughout the project I also sought the expertise of Dr. Gareth Alexander, who helped with the formal definitions of the topological operations and algorithms needed to obtain our novel findings. I have run the simulations and analysed the data, while all authors have contributed to write the paper.

The content of papers (4) and (5) is described in Chapter 5 and Appendix C. The idea behind these papers came to me when looking for an example of topologically linked rings in biology. Once again, I sought the collaboration and expertise of Dr. Marenduzzo and Prof. Orlandini to define the coarse-grained numerical model that could best reproduce the biological system under study. An analytical attempt to describe this system (paper (5)) was originated while I was describing paper (4) to Prof. Turner.

The material published in papers (3) and (6) is reported in Chapter 6 and Appendixes D and E. The ideas contained in paper (3) and the first part of Chapter 6 have originated as a continuation of paper (1) from the regular discussions I had during the preparation of paper (1) with Prof. Turner and Prof. Orlandini. The analytical tools used to recover the non-equilibrium equation for the mobility of the polymers have been suggested by Dr. Baiesi during few meetings I had with him when visiting Prof. Orlandini in Padova. The second part of Chapter 6 describes the results published in paper (6). These were obtained from simulations that I run based on ideas originated from discussions with Dr. Marenduzzo on gel electrophoresis, again in collaboration with Prof. Orlandini, who contributed with his expertise on knot detection algorithms and the preparation of knotted configurations. 


\section{Abstract}

$\mathrm{R}$ ING polymers offer a richness of behaviours that are of broad interest and have deep consequences in many fields of Science. In this Thesis I inchemical nature of the polymers, emerging from systems made of a collection of rings. These will be studied by using methods of equilibrium and non-equilibrium Statistical Mechanics together with Molecular Dynamics and Monte Carlo simulations of coarse-grained models for the systems under investigation. Within these frameworks, important questions regarding the macroscopic behaviour of ring-shaped polymers have yet to find a satisfactory answer. The work presented in this Thesis finds its principal motivations in problems arising in Material Science, the so called "melt" of rings, and in Biology, such as the organisation of mitochondrial DNA in some organisms and the mechanisms governing the electrophoretic separation of DNA samples in gels. There are several theoretical challenges in these fields which represent state-of-the-art scientific research and whose partial answers are provided in the work presented in this Thesis. One of the major achievements of the work presented is the general understanding of the role played by topological properties, i.e. those invariant under smooth deformations of the polymer contour, on the macroscopic behaviour of the investigated systems. Finally, the conclusions drawn from the presented work can have important scientific consequences as they may ultimately lead to a more complete understanding of complicated issues in Biology and to the design of next-generation soft materials. 


\section{List of Symbols}

$\bar{\rho}_{p} \quad$ Percolation monomer density for a system of linkable rings confined in a box. The estimated percolation density is denoted as $\rho_{p}$.

$\beta \quad$ "Surface exponent" relating the number of beads that are in contact with any other bead to the polymer length.

$\eta \quad$ Solution viscosity.

$\gamma \quad$ "Contact exponent" regulating the probability of two parts of the same chain, $s$ segments apart, to be near one another.

$\langle k\rangle \quad$ Average network valence or mean vertex degree.

$\langle l\rangle \quad$ Average fraction of linearised mini-circles.

$\mu \quad$ Mobility of an object dragged by an external force $F$ through a fluid with a stationary velocity $v$.

$\mu_{D} \quad$ Differential mobility, i.e. the change in mobility in response to a change in the field strength.

$\nu \quad$ Scaling exponent relating the spatial size of a polymer to its polymerisation index.

$\phi \quad$ Volume fraction.

$\Phi_{M / D / T}$ Fraction of monomers (one ring), dimers (two linked rings) and trimers (three linked rings) after digestion of a network of linked rings.

$\rho \quad$ Number density of polymer beads in the system. $\rho^{*}$ denotes the overlap number density.

$\sigma \quad$ Nominal diameter of a bead forming the polymers.

$\tau_{\text {diam }}$ Polymer re-orientation time. Quantifies the time taken by a polymer to rearrange its conformation. It is obtained as a numerical time integral of the time-correlation function $C_{\text {diam }}(t)$ computed from $\langle\boldsymbol{d}(t) \cdot \boldsymbol{d}(0)\rangle$, where $\boldsymbol{d}$ is the diameter vector of the polymer.

$\tau_{\text {relax }}$ Polymer relaxation time, or time required for the centre of mass of a polymer coil to diffuse a distance equal to the polymer average size $\left\langle R_{g}^{2}\right\rangle^{1 / 2}$.

$\tau_{\text {Rouse }}$ Rouse time, or time required for a bead forming a polymer to diffuse a distance equal to the polymer average size $\left\langle R_{g}^{2}\right\rangle^{1 / 2}$.

$\varphi_{\mathrm{nc}}(t)$ Time correlation function for contiguous rings. The time-scale at which two rings become non-contiguous is denoted by $\tau_{\mathrm{nc}}$. 
$\xi \quad$ Friction coefficient acting on a bead.

$b_{1}(G)$ First Betti number, or rank of the first homology group, of the graph $G$. It measures the number of 1-dimensional holes in the graph.

$c \quad$ Concentration of polymeric mass in the system. $c^{*}$ denotes the overlap concentration.

$c_{p} \quad$ Fraction of explicitly immobilised (pinned) rings. The critical fraction at which the whole system displays a dynamical arrest is denoted with $c_{p}^{\dagger}$.

$D_{C M}$ Diffusion coefficient of a polymer centre of mass.

$d_{i j} \quad$ Distance between bead $i$ and bead $j$.

$G(\mathcal{E}, \mathcal{V})$ Directed graph composed by a set of vertices $\mathcal{V}$ and edges $\mathcal{E}$.

$G(t) \quad$ Stress relaxation modulus.

$g_{1}(t)$ Segmental mean square displacement of the beads, also identified with $\left\langle\delta r_{s}^{2}(t)\right\rangle$.

$g_{3}(t)$ Mean square displacement of the centre of mass of the polymers, also identified with $\left\langle\delta r_{C M}^{2}(t)\right\rangle$.

$L \quad$ Linear dimension of the simulation box.

$l_{p} \quad$ Persistence length of a semi-flexible polymer, i.e. the contour-length necessary for the tangent-tangent correlation to equal $1 / e$. The Kuhn length is denoted by $l_{K}$ and is twice the persistence length.

$M \quad$ Polymer number-weighted polymerisation index, i.e. number of beads forming the polymers.

$m \quad$ Mass of a bead.

$M_{e} \quad$ Entanglement length, or number of beads at which a polymer starts to "feel" the presence of entanglement due to the neighbouring chains.

$N \quad$ Number of polymers in the system.

$P_{p}(t)$ Threading time-correlation function. It quantifies the time required for the passive threadings observed at any time-step to disappear and release the constraint imposed.

$P_{I J}(t)$ Matrix element identifying the "contact" of ring $I$ and $\operatorname{ring} J$ at time $t$.

$p_{t h} \quad$ Threading probability between two chains sharing the same gel unit cell.

$R_{e}, R_{g}$ End-to-end size and radius of gyration of a polymer coil.

$S_{1}(q)$ Static scattering function probed at length-scales $l=2 \pi / q$.

$S_{c}(q, t)$ Coherent scattering function probed at length-scales $l=2 \pi / q$ at time $t$.

$T h(i, j ; t)$ Matrix element which assumes value of unity if ring $j$ is passing through ring $i$ at time $t$ and zero otherwise.

q Effective charge of a bead. 
lo stimo più il trovar un vero,

benché di cosa leggiera,

che'l disputar lungamente

delle massime questioni

senza conseguir verità nissuna. ${ }^{\dagger}$

G. Galilei

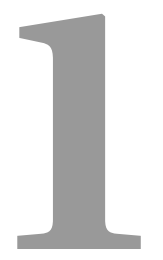

Introduction

口

ING polymers are perhaps one of the last big mysteries in Polymer Physics.

Polymers are collections of simpler units, called monomers, which are nowa-

L days largely present in virtually any aspect of anyone's life: in plastics, drugs and clothes. Perhaps most importantly, (bio-)polymers are at the heart of life itself, being inside any prokaryotic and eukaryotic cell under the form of DNA, RNA and proteins Alberts et al., 2014.

The behaviour of linear polymers in solution is now understood in terms of the Rouse-Zimm and reptation models for dilute and concentrated conditions, respectively de Gennes, 1979, Doi and Edwards, 1988, Rubinstein and Colby, 2003. The behaviour of solutions of star, or quenched branched, polymers is also captured by describing the retraction of the arms similarly to that of the two terminal segments of linear polymers. On the contrary, the properties of un-linked and un-knotted ring polymers in solution present some big open questions and are far from being fully captured. Adding further topological constraints such as, linking between rings or knotting (see Fig. 1.1), complicates the picture even more. In these cases, in fact, the scientific community is not even equipped with the right mathematical tools to classify these objects: At present, a quantity does not exist that can unambiguously distinguish every knot or link Adams, 1994.

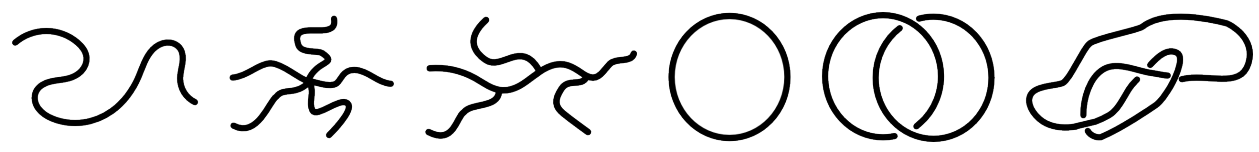

Figure 1.1: Polymers with different topologies. From left to right: linear, star, branched (quenched), circular or ring, linked and knotted.

$\dagger$ I prefer finding something true, although of small importance, rather than keep debating on the major issues failing to achieve any truth. 
Joining the two ends of an open chain would seem, at first sight, to be a relatively trivial change; In reality, it has a profound impact on the static and dynamic properties of a polymer Bates and Maxwell, 2005. This procedure in fact changes its topological state, i.e. the state that is preserved under smooth deformations of its contour, and introduces long-ranged constraints on the allowed conformations. The motion of ring polymers is rather different from that of linear polymers and this is entirely due to their lack of ends. While linear polymers explore the surrounding space by retracting and protruding their terminal segments, ring polymers have no ends to retract or protrude but, on the other hand, they can generate any number of temporary double-folded segments which can explore the space as if they were terminal segments. These conformations are sometimes referred to as "lattice animals", or annealed branched polymers, as the branch points are free to move along the polymer contour (see Fig. 1.2(a)). From this, one can understand that the lacking of ends does not necessarily reduce the ability of ring polymers to explore space but rather triggers completely different pathways through which rings re-arrange their shape. Ring polymers have, in some sense, a greater freedom of movement with respect to their linear or quenched branched polymers, although they suffer of much stronger topological constraints due to the fact that they have to preserve their topological state at all times. Because of all this, ring polymers in solution are, at present, a topic of intense debate and lively interest among the Polymer Physics community Kapnistos et al., 2008, Halverson et al., 2011a, Mirny, 2011, Pasquino et al., 2013, Rosa and Everaers, 2014, Grosberg, 2014.

In spite of our difficulties in capturing their behaviour, ring polymers are abundant in Nature, who seems to have no difficulty at all to regulate their properties and topology, often in very delicate and life-depending conditions, such as in the case of bacterial DNA or inside the eukaryotic cell nucleus Calladine et al., 1997, Alberts et al., 2014. For instance, bacterial DNA, which is circular, has to be kept un-knotted and un-linked at all times during mitosis; failing to do so would lead to the death of the organism as its replicated genetic material cannot be separated into the two daughter cells. DNA knots and links have been frequently observed in the genetic material of bacteria, viruses and eukaryotes, since their discovery in the late '70s Liu et al., 1976, Liu et al., 1981, Fairlamb et al., 1978, and because they are so ubiquitous, all organisms have developed special enzymes - called topoisomerases Berger et al., 1996 - whose function is to help untie DNA knots and links. This indicates how the topological regulation in systems of bio-polymers is a serious issue, and learning how Nature deals with it might provide us with fresh means to design next-generation soft materials or to understand and detect genetic diseases Cavalli and Misteli, 2013, Marini et al., 2015.

One of the most studied ensembles involving ring polymers is the so-called "melt" of rings which consists of a dense solution of rings above its glass and/or crystallization temperatures, i.e. a polymer liquid. The reasons for which this 


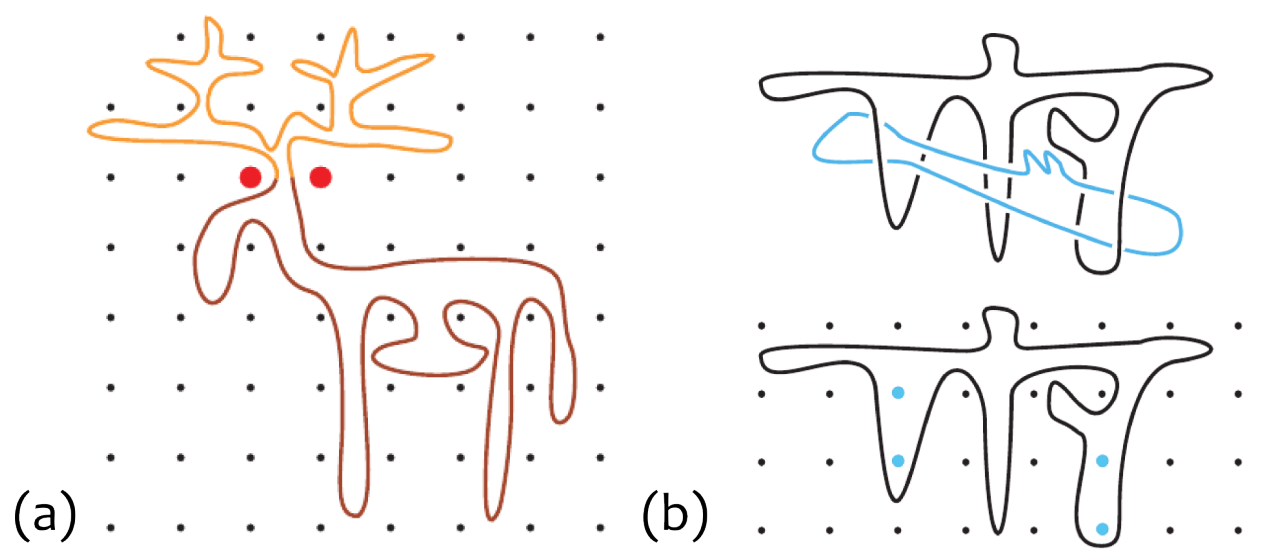

Figure 1.2: (a) A ring polymer that is not threaded by its neighbours (black dots) and assuming a lattice animal (moose) configuration. (b) A ring that is threaded by a neighbour. Its contour can be thought of as encircling points (blue) that cannot be crossed until the blue ring has diffused away (from Ref. Kapnistos et al., 2008).

system has received much attention in the last years are twofold: firstly, it has been found to share some properties with the organisation of chromosomes inside the cell nucleus Cremer and Cremer, 2001, Rosa and Everaers, 2008, Vettorel et al., 2009, Mirny, 2011, Halverson et al., 2011a, Halverson et al., 2011b, Grosberg, 2014, Rosa and Everaers, 2014, Halverson et al., 2014], and secondly, recent experimental advances allowed, for the first time, its purification from linear contaminants which allowed us to study directly the pure melt of rings using artificial polymers such as polyisoprene or polystyrene chains Kapnistos et al., 2008, Pasquino et al., 2013 .

Despite the intense scientific effort spent characterising the dynamics of ring polymers, a full satisfactory description has not yet been achieved. In particular, understanding the conformations assumed by the rings in the melt is of great contemporary interest. Although these show some features reminiscent of a collapsed globule Grosberg et al., 1993 Rosa and Everaers, 2014], they also display some deviations from a fully crumpled conformation Halverson et al., 2011a, and in particular, they show intra- and inter-chain protrusions, also known as "threadings" Lo and Turner, 2013. A ring is said to be passively threaded by another, actively threading, ring when the contour of the former is pierced through by the contour of the latter, very much like the rings in Fig. 1.2(b). These threadings represent serious, although not permanent, constraints on the diffusion of the rings and it has been conjectured that when the number of such constraints is large enough, a spanning cluster of inter-threaded rings might arise and form what has been called a "topological glass" Lo and Turner, 2013. The first part of this Thesis will be devoted to understanding and reproducing (in silico) this peculiar and unique state of matter. The main objectives will be the detection of threadings in melt and dense solutions of ring polymers and their consequent employment as a driving element to tune the physical properties of the system and induce a (topological) kinetically arrested 
state.

The second and third parts of this Thesis will be focused on more biologicallyoriented applications. As mentioned before, knotted and linked ring polymers are abundant in Nature under the form of bio-polymers such as DNA, RNA (or maybe not Micheletti et al., 2015!) and proteins. In order to understand the mechanisms through which their formation and simplification is regulated inside the cell, Molecular Biologists often face the challenge of identifying knots and links topological state. This is nowadays achieved via gel electrophoresis techniques which can efficiently and beautifully separate charged polymers having different length, molecular weight, level of supercoiling and topology or even polymers at different replication stage Calladine et al., 1997, Viovy, 2000, Trigueros et al., 2001, Arsuaga et al., 2002, Olavarrieta et al., 2002. Nonetheless, a physical picture capturing the behaviour of knotted and linked bio-polymers moving through gels in response to external fields as a function of their topological state is still lacking a satisfactory theoretical model. This problem will be tackled in this Thesis from a physical perspective and by focusing on the role of topology in the entangling and disentangling properties of knotted polymers driven by external fields and interacting with random and complex media, such as physical gels.

Another important biological example in which Nature has to deal with polymers displaying topologically complex features is in the case of the mitochondrial genome of organisms of the class Kinetoplastida, also called the "Kinetoplast DNA" Jensen and Englund, 2012]. This is made of thousands of short loops forming a spanning linked network resembling a medieval chain-mail [Chen et al., 1995b. The correct assembly and disassembly of this network during the replicating phase and the corresponding topological regulation is crucial for the survival of this unique species whose evolutionary survival is a long-standing issue in evolutionary biology Borst, 1991. How these organisms can master this complicated task is not at all clear. Although this system presents many complicated biological issues, I will provide a minimal coarse-grained model in order to capture the key elements of the problem and understand the role of topology in this biological system. I will show that, in this case, such simple coarse-grained bio-physical model can not only contribute toward the understanding of how the Kinetoplast is formed and regulated, but it can also provide us with some insight into the evolutionary success of these organisms.

Finally, from the aforementioned examples, one can appreciate that I write this Thesis with the aim of understanding how topology affects the general macroscopic behaviour of systems made of ring (bio-)polymers. In particular, I will focus on three types of topological "interactions", namely threading, knotting and linking, and from each one I will draw and examine specific examples mainly inspired from Material Science and Biology.

It is worth pointing out at this stage that although real-life polymers can be var- 
ious and have the most different roles in our every-day life, ranging from polystyrene which makes common plastics to DNA which contains the information of life itself, they all share some universal physical properties, which are independent of their chemical composition. This is the reason why it is possible to capture the behaviour of such diverse systems within the same coarse-grained physical models. Focussing on the general physical properties of the systems and discarding, as much as possible, any chemical or biological detail can often help us understanding the general underlying mechanisms regulating such systems and pinpoint more general and universal questions.

The work presented in this Thesis will be structured as follows:

In Chapter $2 \mathrm{I}$ will provide the reader with a brief theoretical background on the static and dynamic properties of polymers in solution.

In Chapter 3 I will give a brief general overview of the computational details and numerical schemes used in the rest of the Thesis and will describe the computational models employed.

Chapter 4 will be divided into two sections: First, I will introduce an algorithm to unambiguously detect and identify a peculiar type of topological interaction: threading of un-knotted and un-linked ring polymers. The system I will investigate is a dense solution of ring polymers immersed in gel which will provide me with a way to unambiguously define these elusive kind of inter-chain interactions. In addition, I will investigate the effect of threadings on the relaxation dynamics of the rings and provide an explanation of the observed, both in experiments Doi et al., 2015 and simulations Halverson et al., 2011b, slowing down in terms of emergence of system-spanning connected clusters of inter-threaded rings, thereby relating the increase of spatial correlations with the increase of relaxation time.

In the second part of the Chapter I will tackle a melt of un-knotted and unlinked ring polymers, possibly one of the most studied systems by the Polymer Physics community in recent years. It has been conjectured Lo and Turner, 2013 that the main topological interaction affecting the polymers behaviour in this system is that of threading. On the other hand, currently there is no way to unambiguously identify threadings in a melt of rings, in contrast to the case described before. For this reason I will probe their existence dynamically, i.e. by imposing some external artificial constraints on the rings' motion and by studying the in silico response of the system to this external perturbation. I will introduce a protocol that can provide us with a way to identify the presence of threadings and ultimately show that a melt of rings can, in fact, generate a dynamically arrested state under certain conditions.

In Chapter 5 I will study another type of topological interaction: linking between ring polymers. In order to investigate the properties inherited by a system of rings interacting via linking I will focus on biological organisms whose viability relies on the effectiveness of this topological interaction: the Kinetoplastidae. These 
organisms possess a unique mitochondrial DNA that is made of thousands of linked DNA loops, the "Kinetoplast DNA". I will investigate its structure and provide a simple bio-physical model to explain its stability and topological organisation, both of which are still source of intense debate in the biological community. I will tackle these issues with a philosophy of extreme simplification and the results that I will present will shed some light into the evolutionary advantage of the Kinetoplast and might provide us with some fresh insight into how to artificially generate an "Olympic gel".

In Chapter 6 I will study an intra-chain topological interaction: knotting. As mentioned before, one of the most successful and broadly used techniques to separate knots in biological material is by using gel electrophoresis, although its theoretical understanding is far from complete. For this reason I will focus on understanding how knots interact with the surrounding environment and in particular I will compare linear, circular un-knotted and knotted polymers dragged by an external field through a disordered environment, such as that of a physical gel. I will investigate their behaviour depending on different field strengths, environmental disorder and knot type. The results I will present will be particularly important as they can directly inform biological experiments such as DNA gel electrophoresis and enlighten some recent unexplained experimental outcomes.

Finally, in Chapter 7 I will draw some conclusions, summarise the main findings and attempt to provide a unifying view over the topics treated in this Thesis. 
No man is obliged to learn

and know every thing; [...];

yet all persons are under some obligation

to improve their understanding;

otherwise it will be a barren desert,

or a forest with overgrown weed and brambles.

I. Watts

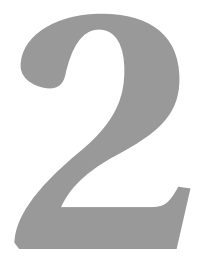

\section{Predicting the Behaviour of Rings in Solution}

\section{Contents}

2.1 Statics .................. 8

2.1.1 The Size of a Crumpled Coil . . . . . . . . . . 8

2.1.2 Contact Exponents for the Crumpled Globule . . . . . . . 13

2.1.3 The Structure Factor . . . . . . . . . . . . . . . . 15

2.2 Dynamics .................... 16

2.2.1 Diffusion Coefficient and Relaxation Time . . . . . . . . 16

2.2.2 How Rings Relax Stress . . . . . . . . . . . . . . . . . 19

2.2.3 Inter-Coil Correlations Probed by Dynamic Scattering . . . 20

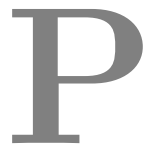

OLYMERIC systems offer an incredible richness of behaviour. Depending on the solution concentration, its temperature or its quality and the polymers length, or topology, every system made of polymers can be categorised into a "universality class", within which it finds a physical characterisation (scaling) of its macroscopic properties.

The physical properties of polymers have been pioneered by Flory in the ' 50 s, by Edwards in the '60s and '70s and by de Gennes in the '70s and '80s. The theories that they developed were mainly concerned with linear polymers and helped the understanding and realisation of many polymer compounds used nowadays. Both Edwards and de Gennes became, at some stage, interested in studying polymers displaying more complicated topologies. They mainly focused on branched and star polymers, although both of them turned their attention to ring polymers, sooner or later, during their lifetime. Edwards Edwards, 1967, Edwards, 1968] chose to tackle the matter from a field-theoretic point-of-view while de Gennes de Gennes, 1979, Raphael et al., 1997] chose a more practical "gedankenexperiment" in which he studied a gel made of linked polymer rings, broadly known as "Olympic gel". Both these series of attempts were far from being the most successful and important 
contributions brought forward by these two giants which indicates the difficulty of the topic (and partially excuses the diffident approach that I will assume in tackling the matter). Even though understanding ring polymers is a difficult task, important advances in the field have been achieved in the past decades Cates and Deutsch, 1986, Rubinstein, 1986, Grosberg et al., 1993, Obukhov and Rubinstein, 1994]: Ring polymers are nowadays well known for behaving very differently from their linear counterparts, although a full theoretical description of their staic and dynamic properties is far from achieved.

In this Chapter I will briefly review the main theoretical findings regarding systems of ring polymers in dense solutions, melts and embedded in gels. In particular, I will separately treat static and dynamic properties, and I will introduce the key observables that I will use to characterise and investigate the systems in the following Chapters.

\section{$2.1 \quad$ Statics}

\subsubsection{The Size of a Crumpled Coil}

The size of a polymer coil has been investigated in various solvents and different concentrations in the past decades. The general assumption is that the size $R$ of a polymer coil depends on the degree of polymerisation $M$ as

$$
R \sim M^{\nu}
$$

where $\nu$ is also known as the entropic exponent and is related to the fractal dimension of the coil via $\nu=d_{F}^{-1}$. The Gaussian, or ideal, approximation for the end-to-end size $R_{e}$ of a polymer coil results in the scaling

$$
R_{e}^{2}=\sum_{i, j}^{M}\left\langle\boldsymbol{r}_{i} \boldsymbol{r}_{j}\right\rangle=\sum_{i}^{M}\left\langle\boldsymbol{r}_{i}^{2}\right\rangle+\sum_{i \neq j}^{M}\left\langle\boldsymbol{r}_{i} \boldsymbol{r}_{j}\right\rangle=M \sigma^{2},
$$

where $\boldsymbol{r}$ is the vector joining two consecutive segments along the chain, $\left|\boldsymbol{r}_{i}\right|=\sigma$ is the size of a segment and segments $\boldsymbol{r}_{i}, \boldsymbol{r}_{j}$ are correlated only if $i=\sqrt{ }$. This gives the value of $\nu=1 / 2$ for ideal coils and applies to polymers in dimension $d$ above the upper critical dimension $d_{c}=4$, for which the ideal polymer picture breaks down and self-avoiding (steric) constraints become too important to be neglected.

One of the most famous and important schemes to infer the value of $\nu$ for selfavoiding polymer coils in $d<d_{c}$ has been advanced by Flory Flory, 1953: Given the monomer concentration of a coil

$$
c_{\text {int }} \simeq \frac{M}{R^{d}}
$$

\footnotetext{
*Local (short-ranged) correlations, do not affect the scaling.
} 
the steric repulsion inside a polymer coil of volume $R^{d}$ can be given in a mean-field picture, i.e. neglecting the inter-monomer correlations, as a virial term

$$
F_{\text {steric }} \simeq k_{B} T v c_{i n t}^{2} R^{d} \simeq k_{B} T v \frac{M^{2}}{R^{d}}
$$

where $v$ takes the role of excluded volume parameter ( $v>0$ for good solvents) and has dimensions of a $d$-dimensional length. This repulsive term is balanced by an entropic term which contrasts the coil expansion much further than the ideal size (with $\nu=1 / 2$ ). This entropic term can be written as

$$
F_{\text {elastic }} \simeq k_{B} T \frac{R^{2}}{M \sigma^{2}}
$$

Summing both terms, the free energy of a coil can be written as

$$
\frac{F}{k_{B} T}=\frac{F_{\text {steric }}+F_{\text {elastic }}}{k_{B} T} \simeq v \frac{M^{2}}{R^{d}}+\frac{R^{2}}{M \sigma^{2}}
$$

After minimisation in terms of the size $R$, this formula leads to the famous scaling for self-avoiding coils in $d$ dimensions

$$
R \simeq\left[v \sigma^{2} N^{3}\right]^{1 /(d+2)}
$$

which gives $\nu_{1 D}=1, \nu_{2 D}=3 / 4$ and $\nu_{3 D}=3 / 5$, which surprisingly $\left.\right|^{\dagger}$ well agrees with experimental observations (in particular numerical estimates give $\nu_{3 D}=0.588$ ).

The size of a ring polymer, because of its lacking of ends, is better captured by the radius of gyration, defined as

$$
R_{g}^{2}=\frac{1}{2 M^{2}} \sum_{i, j}\left[\boldsymbol{R}_{i}-\boldsymbol{R}_{j}\right]^{2}=\frac{1}{M} \sum_{i}\left[\boldsymbol{R}_{i}-\boldsymbol{R}_{C M}\right]^{2}
$$

where $\boldsymbol{R}_{i}$ is the position of segment $i$ and $\boldsymbol{R}_{C M}$ is the position of the ring's centre of mass. Nonetheless, the same Flory theory applies to rings, which follow the scaling $R_{g} \sim \sigma M^{\nu}$ with $\nu=3 / 5$ in $3 \mathrm{D}$ and in good solvent in dilute conditions.

When rings are placed inside a gel structure the picture changes dramatically. In fact, while linear polymers remain in the same universality class, i.e. retain the same $\nu$ when placed inside a gel, rings have been shown to completely change their behaviour. Rings embedded in a gel whose lattice spacing is less than or comparable to the rings persistence length $l_{P}$ (i.e. the length needed to de-correlate the tangent vector or, equivalently, to observe spontaneous bending due to thermal fluctuations) assume lattice animal (LA) configurations. Examples of these are depicted in Fig. 1.2. The rings assume double-folded configurations to preserve their topol-

\footnotetext{
†"Surprisingly" because Flory's theory actually overestimates the repulsive term by neglecting monomer-monomer correlations, but also overestimates the elastic term, thereby balancing out the errors and leading to a very accurate estimation of the scaling of the real size $R$ de Gennes, 1979.
} 
ogy and by doing this they protrude through the gel pores via temporary loops, or branches. The first prediction of the scaling exponent in $d$ dimensions of such random, or annealed, branched structures was given by Lubensky and Isaacson Lubensky and Isaacson, 1979, Isaacson and Lubensky, 1980 and, subsequently, by Parisi and Sourlas Parisi and Sourlas, 1981 in terms of the exponent of the Lee-Yang edge singularity of the Ising model in $d-2$ dimensions and it is, to my knowledge, one of the few (if not the only) exact field-theoretic result in 3 dimensions, and gives

$$
R_{g} \sim \sigma M^{5 /[2(d+2)]}
$$

This prediction, which holds for isolated self-avoiding ring polymers in gels, or lattice animals, in dimensions $d<d_{c}=8$, is (in 3D: $\nu=1 / 2$ ) incidentally the same as that for ideal chains, although the statistics of the conformations is completely different. It is also worth noting that annealed and quenched branched polymers are in different universality classes Gutin et al., 1993. This means that branched polymers with fixed, i.e. quenched, functional units (or branching points), do not behave like lattice animals, for which the branching point are temporary, i.e. can be annealed.

What happens when the coils are instead in concentrated conditions, i.e. many different chains are overlapping and $c>c^{*} \simeq M / R^{d}$ ? In this case, it is well known de Gennes, 1979, Doi and Edwards, 1988 that the steric interaction between different coils screens the coils self-avoidance. This means that in a melt of polymers, i.e. a dense solution of polymers from which the solvent has been drained, the statistics of the polymers is ideal once again, i.e. as if $d \geq d_{c}$. In this case linear polymers return to assume $\nu=1 / 2$, but what happens to the rings? One could argue that a ring polymer in the melt should assume the size of an ideal ring polymer, in agreement with the behaviour of linear polymers in melt. This is not true. In fact, the size of an ideal annealed branched polymer can be described in terms of ideal tree-like structures and its scaling is given by the Kramers theorem Daoud and Joanny, 1981, Rubinstein and Colby, 2003

$$
R_{g}^{2}=\frac{\sigma^{2}}{M} \frac{\sum_{k} M_{1}(k)\left[M-M_{1}(k)\right] Z_{M_{1}(k)} Z_{M-M_{1}(k)}}{\sum_{k} Z_{M_{1}(k)} Z_{M-M_{1}(k)}} \sim \sigma^{2} M^{1 / 2},
$$

where the sum is over all the bonds $k$ which separate the branched tree into two sub-trees (always the case as there are no loops in lattice animals) of sizes $M_{1}$ and $M-M_{1}$ weighted by the corresponding probability $Z_{M_{1}(k)} Z_{M-M_{1}(k)}$, and which gives $R_{g} \sim \sigma M^{1 / 4}$. On the other hand, this prediction is only valid for dimensions $d \geq d_{c}=8$ de Gennes, 1979, Isaacson and Lubensky, 1980 and, in particular, in $d=3$ generates a clear artificial singularity in the coil mass as the degree of polymerisation $M$ increases. For this reason Daoud and Joanny Daoud and Joanny, 1981 conjectured that the limiting scaling for a randomly annealed structure in $d$ dimensions and in concentrated conditions might be obtained from a free energy 

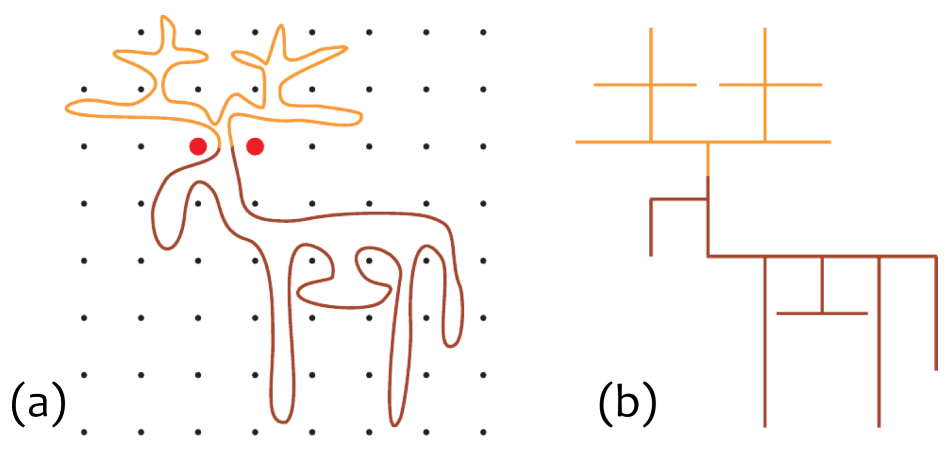

(c)

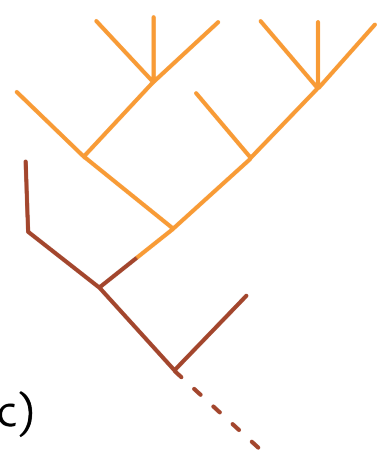

Figure 2.1: (a) The Moose-like configuration of a ring polymer in gel and its tree representation (b) which can be also mapped to a Cayley-tree (partially showed in (c)) whose nodes have maximum valence equal to 4 . Adapted from Kapnistos et al., 2008.

that contains all the $n$-body terms

$$
\frac{F}{k_{B} T}=v \frac{M^{2}}{R^{d}}+w \frac{M^{3}}{R^{2 d}}+\cdots+t_{n} \frac{M^{n}}{R^{d(n-1)}}+\ldots
$$

and whose minimisation (with respect to $R$ ) leads to

$$
R_{g} \sim \sigma M^{1 / d}
$$

once $n \rightarrow \infty$. This scaling has been found, both numerically (see Chapter 4 and Halverson et al., 2011a) and experimentally (see Gooßen et al., 2014, Brás et al., 2014, Doi et al., 2015]), to correctly describe the size of ring polymers in melt, or dense solutions, in the limit of large polymerisation $M$.

More recently, Grosberg Grosberg, 2014 provided a Flory-like theory in order to specifically address and describe the scaling of ring polymers in the melt in terms of their spatial size $R$ and their "Cayley-tree representation" size $L_{c}$. The latter represents the extension of the Cayley tree, or graph, representation of the lattice animal (see Fig. 2.1). If $L_{c} \sim M / 2$ then the ring is fully stretched and all the segments belong to the backbone $L_{c}$. On the other hand, if $L_{c} \sim \ln M$, then the ring resembles a dendritic polymer Grosberg, 2014. By assuming the existence of a further scaling exponent $\rho_{c}$ such that $R \sim \sigma M^{\nu}$ and $L_{c} \sim \sigma M^{\rho_{c}}$ (or $R \sim \sigma^{1-\nu / \rho_{c}} L^{\nu / \rho_{c}}$ ), one can introduce two Flory-like free energies: $F_{\text {elastic }} \sim k_{B} T R^{2} / \sigma^{2} L_{c}$ which penalises the stretching of the backbone, and a second $F_{\text {branching }} \sim k_{B} T L_{c}^{2} / M \sigma^{2}$ which penalises insufficient branching on the Cayley tree. After a rescaling due to the presence of an entanglement length-scale $M_{e}$, which defines a blob size below which the statistics is Gaussian, and that maps to an edge of the Cayley tree representation f $^{\circ}$ one obtains:

$$
\frac{F}{k_{B} T} \sim \frac{R^{2}}{L_{c} \sqrt{M_{e}} \sigma}+\frac{L_{c}^{2}}{M \sigma^{2}}
$$

\footnotetext{
${ }^{\ddagger}$ This means that $M \rightarrow M / M_{e}, \sigma \rightarrow \sigma M_{e}^{1 / 2}$ and $L_{c} \rightarrow L_{c} / M_{e}^{1 / 2}$.
} 
which, after minimisation with respect to $L_{c}$, gives

$$
L_{c} \sim \sigma M_{e}^{1 / 2}\left(\frac{R}{M^{1 / 3} M_{e}^{1 / 6}}\right)^{2 / 3}\left(\frac{M}{M_{e}}\right)^{5 / 9}
$$

For this value of $L_{c}$, the resulting free energy is a monotonic function of $R$ and one can therefore argue that it will attain its minimum at the minimum (physical) value of $R$, i.e. $R \sim \sigma M^{1 / 3}$. This therefore leads to

$$
R \sim\left\{\begin{array}{ll}
\sigma M^{1 / 2} & \text { for } M \ll M_{e} \\
\sigma M_{e}^{1 / 6} M^{1 / 3} & \text { for } M \gg M_{e}
\end{array} \quad \text { or } \nu=1 / 3\right.
$$

and

$$
L_{c} \sim\left\{\begin{array}{lr}
\sigma M^{1 / 2} & \text { for } M \ll M_{e} \\
\sigma M_{e}^{-1 / 18} M^{5 / 9} & \text { for } M \gg M_{e}
\end{array} \quad \text { or } \rho_{c}=5 / 9 .\right.
$$

Equivalently, one finds $R \sim \sigma^{1-\nu / \rho_{c}} L_{c}^{\nu / \rho_{c}}=\sigma^{2 / 5} L_{c}^{3 / 5}$ in the limit of large rings. In summary, within this picture rings in melt can be thought of as fractal globules $R \sim \sigma M^{1 / 3}$ whose backbone is governed by self-avoiding statistics $R \sim \sigma^{2 / 5} L_{c}^{3 / 5}$. While $R$ can easily be obtain, via, for instance, the radius of gyration $R_{g}$, measuring the size of the backbone $L_{c}$ presents much more difficulties and has never been done in the literature. This is because there exists no algorithm able to detect a tree-like structure that is not clearly visible with naked eye, and even primitive-path analysis fails in this case Halverson et al., 2011a.

A further attempt to capture the behaviour of rings in the melt via a Flory-like framework is worth mentioning here. Cates and Deutsch in the '80s Cates and Deutsch, 1986 advanced a model that simply assumes that rings in the melt are topologically constrained by their neighbours and this leads them to adopt a double folded configuration. The constraint experienced by the rings can be associated with an entropic loss of roughly one entropy unit, or degree of freedom $\left(k_{B} T\right)$, per neighbour. Since every chain has roughly $R^{d} / M$ neighbours, one obtains:

$$
\frac{F}{k_{B} T} \sim \frac{R^{d}}{M \sigma^{d}}+\frac{M \sigma^{2}}{R^{2}}
$$

where the second term is the elastic energy required to extend a chain. After the usual minimisation in terms of $R$ one obtains:

$$
R \sim \sigma M^{2 /(d+2)}
$$

which gives, in particular, $\nu_{3 D}=2 / 5$. In Ch. $4 \mathrm{I}$ will show that this prediction is recovered as a crossover between the short chain regime, $R \sim \sigma M^{1 / 2}$, and the long chain limit, $R \sim \sigma M^{1 / 3}$. 


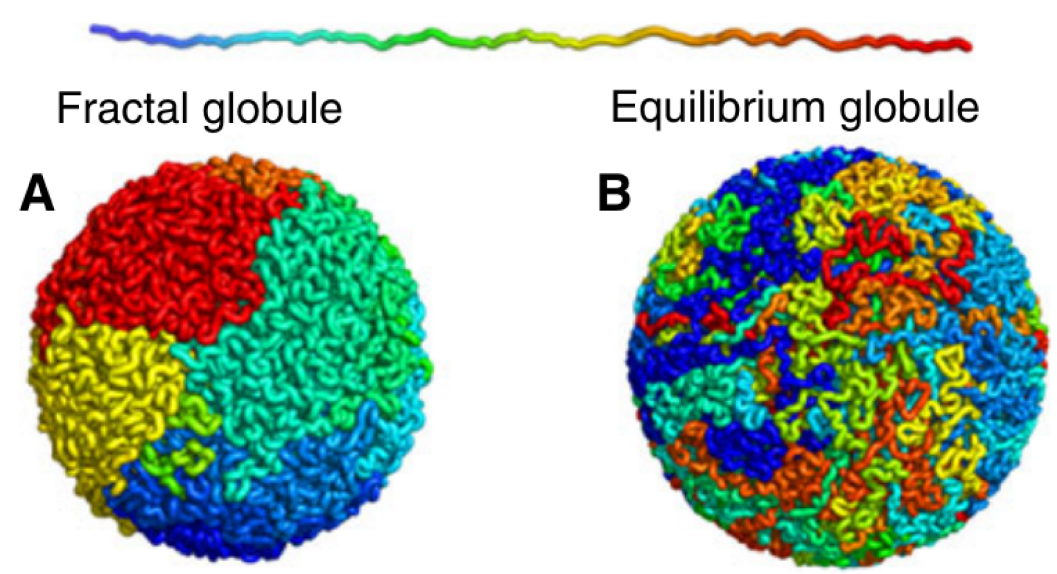

Figure 2.2: Pictorial representation of (a) fractal and (b) equilibrium, globules. While the former displays internal segregation of the segments, the latter displays a larger degree of mixing. These different features are reflected by the different values assumed by the contact exponent, which is $\gamma=1$ for the former and $\gamma=3 / 2$ for the latter (from Ref. [Mirny, 2011]).

Even though ring polymers only differ from their linear counterparts in having a closed contour, they display markedly distinct behaviour. Understanding this, represents one of the most challenging tasks remaining in Polymer Physics. In addition, the so-called "fractal globule" structure often associated with the scaling collapsed state of rings in the melt has raised also some interest in the Biology community. This is because it represents a strongly collapsed state which displays a weak level of entanglement among parts of the sub-chain; as a consequence, it has been identified as a good candidate to explain the structure of chromatin Mirny, 2011 and to describe the formation and the stability of chromosome territories Cremer and Cremer, 2001, Rosa and Everaers, 2008.

\subsubsection{Contact Exponents for the Crumpled Globule}

Although the collapsed conformation assumed by rings in the melt is broadly accepted, it is far from clear what their internal arrangement is. In fact, although $\nu=1 / 3$ resembles a collapsed coil, such as one that could be observed in poor solvent, there are many types of internal arrangements consistent with $\nu=1 / 3$ Rosa and Everaers, 2014. Perhaps the most important candidates in this case are (i) the equilibrium globule: a disordered dense packing of coil confined within a sphere of radius $R \sim M^{1 / 3}$ and possessing a core filled with segments (of length $s<M^{2 / 3}$ ) following ideal statistics, i.e. $r(s) \sim s^{1 / 2}$; and (ii) the fractal globule: a recursive coiling of mass which appears like a collapsed globule at any length scale (larger than the entanglement length $M_{e}$ ) within the globule Grosberg et al., 1993, i.e. $r(s) \sim s^{1 / 3}$ (see Fig. 2.2). One of the key differences between these two conformations is the probability of contact $P_{c}(s)$ of two segments distant $s$ segments along 
the contour and defined as

$$
P_{c}(s)=\left\langle\frac{1}{M} \sum_{i=1}^{M-1} \sum_{j=i+1}^{M} \Theta\left(a-\left|\boldsymbol{r}_{i}-\boldsymbol{r}_{j}\right|\right)\right\rangle
$$

with $a$ a chosen cut-off for the interaction and $\Theta(x)$ the Heaviside function. A mean-field estimate of this probability leads to

$$
P_{c}(s) \simeq \frac{\sigma^{d}}{r(s)^{d}} \sim s^{-\nu d} \equiv s^{-\gamma}= \begin{cases}s^{-3 / 2} & \text { equilibrium globule and } s<M^{2 / 3} \\ s^{-1} & \text { fractal globule }\end{cases}
$$

where $\gamma$ is called the contact exponent. The mean-field value of $\gamma=1$ for the fractal globule case is un-physical, since the number of neighbours per segment would, in this case, diverge as $\sum_{s} s^{-1} \rightarrow \infty$. Numerical Halverson et al., 2011a, Halverson et al., 2013 and experimental Lieberman-Aiden et al., 2009, Mirny, 2011 observations in fact report a contact exponent close to, but slightly greater than, unity.

Another useful quantity is how "rough" the coil surface is. This can also be understood in terms of number of contacts $n_{c}$ or inter-blobs $\$$ contacts $n_{g}$. Let us assign to these two quantities the exponents $\beta_{c}$ and $\beta_{g}$ regulating the scaling of the contacts for an $s$-monomer long sub-chain as

$$
n_{c}(s) \sim s^{\beta_{c}}
$$

being the number of contacts of a given segment with any other segment and

$$
n_{g}(s) \sim s^{\beta_{g}}
$$

being the number of contacts between blobs sitting near one another in space. The surface exponents $\beta_{c}$ and $\beta_{g}$ have to be related to one another by the fact that only a number $r(s)^{d} / s$ of blobs can be neighbours to a given blob, and therefore:

$$
s^{\beta_{c}} \sim \frac{r(s)^{d}}{s} s^{\beta_{g}}=s^{\nu d-1} s^{\beta_{g}}
$$

which gives

$$
\beta_{c}=\beta_{g}+\nu d-1
$$

or in the case of a globule $(\nu=1 / d)$ :

$$
\beta_{c}=\beta_{g}
$$

On the other hand, the contact exponent $\gamma$ is itself related to the surface ex-

\footnotetext{
${ }^{\S}$ A blob being a polymer segment made of several $(g)$ monomers where $1 \ll g \ll M$ and assuming a size described by the scaling $R(g) \sim g^{\nu}$ with $\nu=3 / 5$, being not interacting with other chains de Gennes, 1979.
} 
ponent $\beta_{c}$ as the number of contacts of a given monomer with other monomers is $\sum_{s^{\prime}>s}^{\infty} P_{c}(r)$, which gives the total number of contacts of an $s$-monomer long segment as

$$
s^{\beta_{c}} \sim s \sum_{s^{\prime}=s}^{\infty}\left(s^{\prime}\right)^{-\gamma} \sim s^{-\gamma+2}
$$

or

$$
\beta_{c}+\gamma=2
$$

It is worth stressing that eq. (2.24) and eq. 2.27) are general relations which hold for any fractal structure and do not rely on the fractal globule assumption. Also, from eqs. (2.24) and 2.27) one can infer some restrictions for the values of these exponents, in particular $\beta_{c} \geq \beta_{g}$ and $1 \leq \gamma \leq 1+1 / d$. It is also worth noting that for Hilbert curves in 3D for which $R \sim M^{1 / 3}$ (being space filling), the contact surface scales with $\beta=\beta_{c}=\beta_{g}=2 / 3$ which implies $\gamma=4 / 3$, while, by contrast, the numerical estimation of $\beta$ for the fractal globule case (see Ch. 4 and Halverson et al., 2011a ) seems to be close to unity, $\beta \simeq 0.95-0.98$, implying $\gamma=1.02-1.05$ compatible with the results from Hi-C contact maps LiebermanAiden et al., 2009, Zhang et al., 2012 and the findings reported in Ch. 4 .

\subsubsection{The Structure Factor}

Another observable that is worth investigating in order to probe the internal fractal structure of polymer coils is the single-chain static structure factor, $S_{1}(q)$. Experimentally, this quantity can be measured via light or neutron scattering (refer to Rubinstein and Colby, 2003 for experimental realisation details). This is defined as

$$
S_{1}(q)=\left\langle\frac{1}{M} \sum_{i}^{M} \sum_{j}^{M} e^{i \boldsymbol{q}\left(\boldsymbol{r}_{i}-\boldsymbol{r}_{j}\right)}\right\rangle
$$

where the average is taken over polymers, monomer positions $\boldsymbol{r}_{i}$ and over orientations of the scattering wave-vector $\boldsymbol{q}$ (when the system is isotropic). For wave-vectors $|\boldsymbol{q}|$ much smaller than $R_{g}^{-1}$ (or length-scales $|\boldsymbol{q}|^{-1}$ much larger than $R_{g}$ ), the scattering function gives

$$
S_{1}(q) \simeq M
$$

since all monomers within the coil contribute to the sum. When $2 \pi R_{g}^{-1}<q<$ $2 \pi \sigma^{-1}$, one finds that all monomers $n_{q}$ within the volume $q^{-3}$ contribute to $S_{1}(q)$. In this case, the static structure factor relates to the fractal dimension of the chain as

$$
S_{1}(q) \simeq n_{q} \simeq\left(\frac{1}{q^{d} \sigma^{d}}\right)^{1 / \nu d}=(q \sigma)^{-1 / \nu}=(q \sigma)^{-d_{F}} .
$$

\footnotetext{
I These are obtained by using $1 / d \leq \nu \leq 1,1-1 / d \leq \beta_{c}=\beta_{g} \leq 1$, in eqs. 2.24) and 2.27).
} 
In the case of linear polymers in melt the structure factor correctly returns $d_{F}=2$ for the whole range $2 \pi R_{g}^{-1}<q<2 \pi \sigma^{-1}$ Kremer and Grest, 1990]. In the case of ring polymers in dense solutions the scaling of eq. 2.28) is less unambiguous and it has been conjectured Halverson et al., 2011a that it displays signatures of more complex internal arrangement of the coils (see also Ch. 4).

\subsection{Dynamics}

\subsubsection{Diffusion Coefficient and Relaxation Time}

The first theories describing how ring polymers diffuse through either other chains or a gel have been advanced separately by Cates, Rubinstein and Klein Cates and Deutsch, 1986, Rubinstein, 1986, Klein, 1986. They share the same spirit, i.e. describing the diffusion of rings in the melt assuming that rings can move in an amoebae fashion through the surrounding (static) obstacles by successive protrusions mediated by kink-gas diffusion along the polymer contour de Gennes, 1979. Within this framework, "kinks" or "defects" (or excess of mass along the chain) can diffuse independently along the polymer contour until they stop their diffusion on a segment of the randomly branched ring structure and contribute to the extension of that segment. By assuming that the kinks perform a 1D random walk along the contour, the time required to span a distance $R_{g}$ corresponds to the Rouse time and is computed as

$$
\tau_{\text {Rouse }} \sim M^{2}
$$

The ring can therefore renew its configuration by consecutive kinks diffusion. For a ring made by $M$ segments this takes a time

$$
\tau \sim M \tau_{\text {Rouse }}=M^{3}
$$

or, equivalently, a diffusion coefficient

$$
D_{C M} \sim \frac{R_{g}^{2}}{\tau}=M^{2 \nu-3}
$$

Using the field-theoretic exponent in eq. 2.9$)$, i.e. $\nu=5 /(2 d+4)$, one finally obtains

$$
D_{C M} \sim M^{-(3 d+1) /(d+2)},
$$

which, in 3D, gives $D_{C M} \sim M^{-2}$ roughly compatible with numerical evidence (see Ch. 4. Appendix B and Cates and Deutsch, 1986, Halverson et al., 2011b). Incidentally, this result is the same for linear polymers in melt, since the overall size of isolated self-avoiding rings in a background of obstacles (or gels) is described by $\nu=1 / 2$ in $3 \mathrm{D}$ which is numerically equal to the exponent for linear polymers in melt. If one were to use the fractal globule exponent $\nu=1 / 3$, eq. 2.33 would 
give $D_{C M} \sim M^{-7 / 3}$ which is weaker than the experimentally and computationally observed scaling.

More recently, Milner, Iyer and then Grosberg Iyer and Arya, 2012, Smrek and Grosberg, 2015 advanced several other theories for the diffusion of a ring polymers among other chains, or "fixed obstacles". Milner and Newhall Milner and Newhall, 2010 proposed an approach based on the "centrality" of a node in the lattice animal representation of the ring defined as

$$
\zeta_{k}=\min \left(M_{1}(k), M-M_{1}(k)\right)
$$

where $M_{1}(k)$ is the size of one of the two sub-trees generated by cutting the $k$-th bond of the tree-representation (see Fig. 2.1). They proposed that, analogously to the reptation mechanism in linear polymers, rings undergo diffusion by vacating bonds via the "evaporation" of one of the two sub-trees across that bond. This means that if the centrality $\zeta_{k}$ of bond $k$ is small, one expects its relaxation to be quick. On the other hand, bonds whose removal produce two sub-trees with similar sizes are expected to take the longest to disappear. Kramers theorem can be used to show that the probability distribution of the nodes' centrality $P(\zeta)$ is given by the weights in Kramers theorem in eq. 2.10 Rubinstein and Colby, 2003, appropriately normalised:

$$
P(\zeta)=\frac{Z_{\zeta} Z_{M-\zeta}}{\sum_{\zeta} Z_{\zeta} Z_{M-\zeta}} \simeq \sqrt{\frac{n_{v}-1}{8 \pi\left(n_{v}-2\right)}}\left(\frac{M}{\zeta(M-\zeta)}\right)^{3 / 2}
$$

where $n_{v}$ is the valence of the nodes. This distribution has the feature that most of the nodes have low centrality and therefore relax quickly. One can then proceed by assuming that rings must arrange themselves in order to satisfy such a centrality distribution, and therefore this acts as an effective entropic potential for the rings biasing the mass diffusion as

$$
\beta U_{\zeta}=-\log P(\zeta)
$$

By performing Monte-Carlo simulations of trees diffusing on Bethe lattices, Milner and Newhall finally concluded that the mass accumulated within $i$ generations from a given high-centrality bond scaled as $M(i) \sim i^{a}=i^{1.5-1.7}$ and, as a consequence, the variance of the centrality should scale as the mass diffused in a time $\tau_{\zeta} \sim t^{2}$ or

$$
\left\langle\Delta \zeta^{2}\right\rangle \sim t^{a / 2}=t^{0.75-0.85}
$$

which leads to a total relaxation time for a tree formed by $M$ nodes, corresponding to the time required for the centrality to diffuse a "distance" of order $M$, of

$$
\tau_{\text {relax }} \sim M^{2 /(a / 2)} \simeq M^{8 / 3}
$$


Their result is again roughly compatible with recent findings Kapnistos et al., 2008, Halverson et al., 2011b, although completely neglects the motion of other chains and, in particular, inter-chain interactions which can explain the even more recent findings Pasquino et al., 2013, Gooßen et al., 2014, Doi et al., 2015 (see also to Ch. (4).

Smrek and Grosberg Smrek and Grosberg, 2015 based on the novel description of a ring as an annealed tree made of crumpled branches decorating a self-avoiding path on a Cayley tree, i.e. $R \sim \sigma M^{1 / 3}$ and $R \sim \sigma^{2 / 5} L^{3 / 5}$ with $L \sim \sigma M^{5 / 9}$ (or $\nu=1 / 3, \rho=5 / 9$ and $\nu / \rho=3 / 5)$, advanced an alternative picture for the dynamics of rings in the melt. By assuming the existence of an "entanglement length" $M_{e}$ below which the chain is Gaussian, i.e. $R_{\text {blob }} \sim \sigma M_{e}^{1 / 2}$, which is taken as the "blob size", the Rouse time for a blob can be written as

$$
\tau_{\text {blob }} \sim \frac{\zeta_{e} M_{e}^{2} \sigma^{2}}{k_{B} T}
$$

where $\zeta_{e}$ is the effective friction of a blob. The chain is formed by $g=M / M_{e}$ blobs and every time a blob moves by $R_{\text {blob }}$ a fraction $1 / g$ of mass contributes to the overall diffusion of the centre of mass. The displacement of the chain center of mass, defined as

$$
\left\langle\delta r_{C M}^{2}\right\rangle \equiv g_{3}(t)=\left\langle\left[\boldsymbol{r}_{C M}\left(t+t_{0}\right)-\boldsymbol{r}_{C M}\left(t_{0}\right)\right]^{2}\right\rangle_{t_{0}}
$$

where $\langle\ldots\rangle_{t_{0}}$ indicates average over different initial time-steps, can therefore be written as the displacement of $g^{\rho}$ blobs of size $R_{\text {blob }}$ forming the backbone $L$ :

$$
\left\langle\delta r_{C M}^{2}\right\rangle \equiv g_{3} \simeq g^{\rho}\left(\frac{\sigma M_{e}^{1 / 2}}{g}\right)^{2}=M_{e} g^{\rho-2} \sigma^{2}
$$

that gives a diffusion coefficient of the blobs along the backbone of $D_{\mathrm{bb}} \sim g_{3} / \tau_{\mathrm{blob}}$. The full relaxation of the chain can be achieved once the centre of mass has travelled the ring's size $L$, i.e.

$$
\tau_{\text {relax }} \sim \frac{L^{2}}{D_{\mathrm{bb}}}=\tau_{\mathrm{blob}} g^{\rho+2}
$$

where one uses the fact that $L \sim M_{e}^{1 / 2} g^{\rho} \sigma$ giving

$$
\tau_{\text {relax }} \sim \frac{\zeta_{e} \sigma^{2}}{k_{B} T} M^{23 / 9}
$$

The diffusion coefficient of the whole ring can be found by imposing that the ring is displaced a distance equal to its own size $\left(R=\sigma M_{e}^{1 / 2} g^{\nu}\right)$ in the relaxation time $\tau_{\text {relax }}$, i.e.

$$
D_{C M} \equiv \frac{R^{2}}{\tau_{\text {relax }}} \sim \frac{M_{e} \sigma^{2}}{\tau_{\text {blob }}} g^{2 \nu-\rho-2} \simeq D_{\text {blob }} M^{-17 / 9}
$$

All these findings are roughly compatible with numerical and experimental ev- 
idence, although they predict exponents that underestimate the observed ones. In particular, none of these explain why the sub-diffusion of the rings can be observed on length-scales many times the ring's gyration radius Halverson et al., 2011b, Halverson et al., 2014. This may be due to chains moving and interacting non-trivially with one-another. The main reason for this is that mean-field theories analyse the behaviour of chains among other "fixed" chains. This is not the case in ring polymer melts where inter-chain interactions are important (see Ch. 4 and Halverson et al., 2011a ) and lead to collective behaviour. In order to correctly capture the dynamics of rings in the melt one should take into account the collective re-arrangements and configurational fluctuations, which makes the problem much harder to tackle.

\subsubsection{How Rings Relax Stress}

How rings relax their stress is perhaps one the key questions that I will try address. Recently there have been quite a few attempts to quantify the stress relaxation of rings in a background of obstacles Milner and Newhall, 2010 and in the melt Kapnistos et al., 2008, Pasquino et al., 2013, Smrek and Grosberg, 2015. All the theoretical effort has been focused on describing rings as amoebae Rubinstein, 1986 moving through obstacles situated around the ring double-folded configuration, i.e. not threading its contour. This strong assumption seems now ubiquitous when tackling the ring melt problem. On the contrary, I will show for the first time that this is instead not a correct assumption: Rings do protrude through one-another, and this has strong consequences on the dynamics, which should be considered when formulating a theory of their stress relaxation (see Ch. 4).

Experimentally there is several evidence that melts of rings display a very low stress-relaxation modulus $G(t)$ which never exhibits a plateau Kapnistos et al., 2008, Pasquino et al., 2013. One the other hand, their slow overall relaxation to free diffusion Halverson et al., 2011b as well as a dramatic viscosity enhancement observed in more recent findings Doi et al., 2015 indicate that inter-coil interactions are important but hard to quantify.

It is very likely, that rings can relax their "internal" (or "configurational"), i.e. intra-coil, stress much faster than they can relax their inter-coil correlations. This conjecture will be supported by computational evidence in Ch. 4. Although achieving an analytical description of this behaviour is not an easy task, I believe that numerically probing the decoupling between relaxation time-scales related to different length-scales can help the theoretical community to formulate a more appropriate description for the motion of rings in the melt or dense solutions. In addition, the possibility that additional "relevant length-scales" exist and directly control the motion of rings in the melt has been raised in the past by Cates and collaborators Müller et al., 1996, Muller et al., 2000 and might have found a direct proof in the results that I will present in Ch. 4 . 
While the internal relaxation of linear polymers is intimately related to the persistence of their neighbours forming the surrounding tube, this cannot be said for ring polymers, which can create new protrusion anywhere along their contour, being not limited by the presence of fixed ends. On the other hand, threadings, which are only possible between ring polymers, might affect the overall relaxation, i.e. the diffusion of the centre of mass of the polymers, leaving the internal stress relaxation mechanisms unaffected. In light of this I propose to focus on measuring the longtime inter-coil correlations which can be most readily done via scattering methods, and in particular via the coherent scattering function $S_{c}(q, t)$, defined below.

\subsubsection{Inter-Coil Correlations Probed by Dynamic Scattering}

Intra-coil correlations on length-scales $l=2 \pi q^{-1}$ are commonly probed by the coherent (or in-coherent) dynamic scattering function $S_{c}(q, t)$ (or $S_{i n}(q, t)$ obtained setting $i=j$ ):

$$
S_{c}(q, t)=\left\langle\frac{1}{M} \sum_{i}^{M} \sum_{j}^{M} e^{i \boldsymbol{q}\left(\boldsymbol{r}_{i}\left(t+t_{0}\right)-\boldsymbol{r}_{j}\left(t_{0}\right)\right)}\right\rangle,
$$

where the average is taken over the rings in the system and different $t_{0}$. In practice, one can imagine one probe chain in the solution scattering the incident light and then repeating the measurement over many different probe chains. This function is also sometimes called the "self-intermediate scattering function" and its Fourier transform the "self-part of the Van Hove function" in the glass-transition community Berthier and Biroli, 2011], where it is one of the main tools used to capture density-density correlations in a glass-forming systems. This scattering function was also studied by de Gennes de Gennes, 1981 to capture the behaviour of one reptating linear chain among other fixed chains.

In some cases, especially when dealing with molecular liquids or liquids made of simple constituents, the dynamic scattering function can give some information regarding inter-objects correlations, being internal degrees of freedom not included in the picture. In the case of polymer liquids, characterising inter-coil correlations via the dynamic scattering function can be less straightforward Aichele and Baschnagel, 2001 Frey et al., 2015. In particular, it is sometimes necessary to extend the computation of eq. (2.46) to all the atoms in the system, rather than the ones forming the chains In this case the function is also known as the (dynamic) "pair-correlation" function, which is rarely studied as numerically infeasible to compute throughout the simulation.

In light of this one is often limited to the computation of eq. 2.46 over the beads forming a single chain and to take the average over many chains. This means that $S_{c}(q, t)$ formally captures intra-coil correlations, and does not give direct evidence

\footnotetext{
"In this case the computation can scale as $(N M)^{2}$, for a system of $N$ chains $M$ beads long, rather than $N M^{2}$.
} 
of inter-coil correlations. On the other hand, it is possible to infer some information on the inter-coil correlations when $q$ becomes greater than the inter-coil distance $2 \lambda$, where $\lambda$ is defined as

$$
\lambda=\phi^{-1 / 3} R_{g}
$$

where $\phi$ is the coils volume concentration, i.e.

$$
\phi=\frac{4 N \pi R_{g}^{3}}{3 \sigma^{3} L^{3}}
$$

The free volume available to the coils is $1 / \phi$ and hence the free length (in units of the radius of gyration of the polymers) is $\phi^{-1 / 3}$. This means that above overlap the inter-coil distance is smaller than $2 R_{g}$.

The information that one can obtain from $S_{c}(q, t)$ on the collective behaviour of the rings is based on the following reasoning: the dominant contribution to $S_{c}(q, t)$ comes from beads $j$ that have not travelled (much) further than $2 \pi / q$ from the other bead $i$ within $t$ time-steps. Therefore if there was a length-scale above which the independent motion of the beads was somehow constrained, that would appear when probing the system with the right $q$. In particular, one can imagine that if a ring was permanently pinned down by a frozen obstacle threading through its contour then one should expect

$$
\lim _{t \rightarrow \infty} S_{c}\left(q \simeq \pi R_{g}^{-1}, t\right)=S_{c}^{\infty}\left(q \simeq \pi R_{g}^{-1}\right)>0
$$

where $S_{c}^{\infty}(q)$ is a constant greater than zero and near one, as most of the beads forming the chain would be forever trapped in a region of linear size $l \simeq 2 R_{g}$. On the other hand, if one was to probe larger $q$ 's, i.e. shorter length scales, one should in principle observe a more unconstrained relaxation, and in particular

$$
\lim _{t \rightarrow \infty} S_{c}\left(q \gtrsim \pi R_{g}^{-1}, t\right) \simeq 0
$$

Although it is worth bearing in mind that this scattering function would not decay strictly to zero, as presence of permanent obstacles, i.e. regions that the beads are not free to explore, has the effect of suppressing the full de-correlation of the monomers.

Even in the case of multiple threadings, the cage formed by the penetrations represent a severe obstacle only for the overall diffusion, while internal modes, shorter than the average distance between threadings, are left unhindered. This suggests that in the case that the threadings are sparse enough, the ring should display a slow decorrelation of the dynamic scattering function on length-scales comparable to the diameter of the ring, but a faster de-correlation on shorter length scales. Extending this reasoning to the case where the pinning ring is itself mobile, one can, in any case, expect the passively threaded ring to be slower (again on length-scales 
$2 \pi q^{-1} \gtrsim 2 R_{g}$ ) compared to a ring that is instead not threaded. Both, threaded and non-threaded rings, should instead relax their internal (i.e. $2 \pi q^{-1} \lesssim 2 R_{g}$ ) stress at roughly the same rate, i.e. having the same decay of $S_{c}\left(q \gtrsim \pi R_{g}^{-1}, t\right)$.

Investigating the differences/analogies in the behaviour of the dynamic scattering function at different length-scales can therefore give some insight into the presence of topological constraints in solutions of rings and can shed some light onto the dynamics of rings. This will be discussed in more detail in Ch. 4 . 
It is nice to know that the computer understands the problem ...

But I would like to understand it too.

\section{E. Wigner}

\section{2} Molecular Dynamics Models

\section{Contents}

3.1 Molecular Dynamics Scheme . . . . . . . . . 24

3.1.1 Non-Bonded Potentials . . . . . . . . . . . . . . . 25

3.1 .2 Bonded Potentials . . . . . . . . . . . . . . 26

3.1 .3 Brownian Dynamics . . . . . . . . . . . . . 27 27

3.2 Modelling .................. 30

3.2.1 Modelling (Knotted) Ring Polymers . . . . . . . . . . . . 31

3.2 .2 Modelling a Physical Gel . . . . . . . . . . . . 355

OMPUTER simulations, or "experiments" Frenkel and Smit, 2001, are important tools for studying complex systems. This Thesis itself largely relies on computational methods, in particular Molecular Dynamics (MD) simulations. For this reason, I devote this chapter to describing the essence of the MD simulations employed here and the computational details of the models described in the subsequent Chapters.

Molecular Dynamics simulations have been used for the first time in the late 50's [Alder and Wainwright, 1959]. They started as a method to investigate the properties of systems of hard spheres Alder and Wainwright, 1957 and simple liquids Rahman, 1964 and later became a fundamental technique to model the dynamics of biomolecules McCammon et al., 1977, Karplus and Petsko, 1990. As opposed to standard Monte-Carlo techniques, MD simulations offer the advantage of naturally probing the dynamical properties of the systems, such as transport coefficients, time-dependent responses and rheological properties. In addition, Molecular Dynamics models are very flexible in terms of the level of coarse-graining performed on the model. They can either be very accurate in describing microscopic molecular details or in evolving a more coarse-grained picture, depending on the level of detail needed. Usually, MD models lend themselves to a much higher level of molecular 
detail, than standard Monte-Carlo models. One of the key challenges of MD models is to include the appropriate inter-molecular potentials, and, in particular, find the right level of coarse-graining required to reach the best accuracy given practical constraints on their feasibility.

Because modelling microscopic chemical interactions are computationally expensive and much of the puzzling physical features of rings in solution are hidden in their long-time behaviour, my interest is in retaining only the key physical elements. I therefore adopt coarse-grained models for the polymers in order to reach longer simulations time-scales. In particular, I will formulate a mesoscopic physical model of the polymers and neglect specific chemical details. Some of the problems discussed in the following Chapters will be naturally associated with specific types of polymers. For instance, gel electrophoresis is very often performed on DNA samples, and therefore it is natural to start from a more physically faithful description for the DNA. On the other hand, the chemical details of the base-pair system is not necessary to capture the physics of gel electrophoresis and will, therefore, be coarse-grained out. In addition, addressing more coarse-grained models has often the advantage of delivering more general results, which might be valid for other systems, as long as they share similar physical and topological properties.

In what follows, I will firstly discuss some general elements of Molecular Dynamics simulations and secondly, I will give describe in detail the coarse-grained models used in the following Chapters.

\subsection{Molecular Dynamics Scheme}

The aim of a Molecular Dynamics simulation is to integrate the classical equations of motion:

$$
\begin{aligned}
\frac{\partial \boldsymbol{r}_{i}}{\partial t} & =\boldsymbol{v}_{i} \\
m_{i} \frac{\partial \boldsymbol{v}_{i}}{\partial t} & =\boldsymbol{F}_{i}=-\frac{\partial \mathcal{U}}{\partial \boldsymbol{r}},
\end{aligned}
$$

where $m_{i}$ and $\boldsymbol{r}_{i}$ are, respectively, the mass and the position of the $i$-th atom, often also referred to as "monomers". $\boldsymbol{F}_{i}$ is the force acting on the $i$-th atom, which can be calculated by knowing the potential energy $\mathcal{U}$. This is, in general, dependent on all the other atoms in the system, and, of course, on external fields applied to the system from the outside. In order to reproduce the correct motion, one therefore needs to define what are the interactions between the atoms in the system under study. These can be classified as: non-bonded and bonded potentials. The former deal with interactions between atoms which are not connected at a molecular level, for instance atoms belonging to different polymers. The latter describe the interactions between atoms which do share a molecular connection, such as hydrogen and oxygen in a water molecule. 


\subsubsection{Non-Bonded Potentials}

Each atom in the simulation can interact via non-bonded potentials with all the other atoms in the system and, if present, a wall delimiting the simulation box. Because of this, one needs to define the 1-body, 2-body, 3-body, etc. interactions as

$$
\mathcal{U}_{n b}=\sum_{i}^{N} u\left(\boldsymbol{r}_{i}\right)+\sum_{i} \sum_{j>i} v\left(\boldsymbol{r}_{i}, \boldsymbol{r}_{j}\right)+\ldots
$$

where $u\left(\boldsymbol{r}_{i}\right)$ is the potential describing the interaction between a single atom and, for instance, a wall and $v\left(\boldsymbol{r}_{i}, \boldsymbol{r}_{j}\right)$ the potential describing a 2-body interaction, e.g. a Lennard-Jones or Coulomb potential. For instance in the case of charged polymers, one should, in principle, include both steric and Coulomb interactions. It is also common, as long as the simulation reproduces the essential physics, to drop all the higher order terms.

The two-body repulsion can be efficiently modelled via the following shiftedtruncated form of the Lennard-Jones (LJ) potential (or Weeks-Chandler-Andersen model Weeks et al., 1971):

$$
\mathcal{U}_{L J}\left(\boldsymbol{r}_{i}, \boldsymbol{r}_{j}\right)=4 \epsilon\left[\left(\frac{\sigma_{c}}{r_{i j}}\right)^{12}-\left(\frac{\sigma_{c}}{r_{i j}}\right)^{6}+\frac{1}{4}\right] \Theta\left(2^{1 / 6} \sigma_{c}-r_{i j}\right)
$$

where $\Theta(x)$ is the usual Heaviside function, i.e. 1 for $x \geq 0$ and 0 otherwise, and $\sigma_{c}$ is the minimum distance between beads. The potential depth is $\epsilon$ and $r_{i j}=\left|\boldsymbol{r}_{i}-\boldsymbol{r}_{j}\right|$ is the distance between the $i$-th and $j$-th atom. This version of the Lennard-Jones potential is chosen in order to broadly model only the steric repulsion between atoms, thereby avoiding (i) unwanted Van der Walls attractions and (ii) long-ranged interactions without introducing discontinuity in the potentials.

Another useful way of modelling steric interactions is via a "soft" potential. One of the most used forms of this potential is the following:

$$
\mathcal{U}_{\text {soft }}\left(\boldsymbol{r}_{i}, \boldsymbol{r}_{j}\right)=\epsilon_{s}\left[1+\cos \left(\frac{\pi r_{i j}}{r_{c}}\right)\right] \Theta\left(r_{c}-r\right)
$$

Here, $\epsilon_{s}$ is the height of the potential at $r_{i j}=0$ and $r_{c}$ the cut-off. This potential is generally used for initialising a system possessing partially overlapping elements. These are in fact gently pushed apart by this potential without generating numerical divergences. This pre-equilibration step is very important to avoid "blow-ups" and it is usually done by performing a short run in which the parameter $\epsilon_{s}$ is slowly raised typically from $\epsilon_{s}=0$ to $\epsilon_{s}=50 \epsilon$, before the LJ potential is turned on. 


\subsubsection{Bonded Potentials}

Bonded potentials describe the interactions between atoms which share molecular bonds. The simplest potential describing the connection between two atoms is the harmonic potential that models the bond as a spring with stiffness $\kappa_{h}$ and equilibrium length $r_{0}$ :

$$
\mathcal{U}_{\text {harm }}\left(\boldsymbol{r}_{i}, \boldsymbol{r}_{j}\right)=\frac{\kappa_{h}}{2}\left[r_{i j}-r_{0}\right]^{2} .
$$

This potential is usually inappropriate for use in polymer chains simulations, especially in dense conditions, as it can allow bonds to stretch and polymers to cross through one-other. More appropriate 2-body bonded potentials exist for system of polymers, such as the Finitely Extensible Non-linear Elastic (FENE) potential:

$$
\mathcal{U}_{\mathrm{FENE}}\left(\boldsymbol{r}_{i}, \boldsymbol{r}_{j}\right)=-\frac{\kappa_{f}}{2} R_{0}^{2} \ln \left[1-\left(\frac{r_{i j}}{R_{0}}\right)^{2}\right],
$$

for $r_{i j}<R_{0}$ and $\mathcal{U}_{\mathrm{FENE}}\left(\boldsymbol{r}_{i}, \boldsymbol{r}_{j}\right)=\infty$, otherwise. The values of the parameters chosen here in this Thesis are $R_{0}=1.6 \sigma$ and $\kappa_{f}=30 \epsilon / \sigma^{2}$. These choices ensure that strand-crossing events are suppressed. This is of paramount importance when the topological state of a polymer, e.g. (un-)knotted or (un-)linked, needs to be preserved throughout the simulation.

In order to model the chains stiffness, the following 3-body bonded potential is commonly used:

$$
\mathcal{U}_{\text {bend }}\left(\boldsymbol{r}_{i}, \boldsymbol{r}_{j}, \boldsymbol{r}_{k}\right)=\frac{k_{B} T l_{p}}{\sigma}\left[1-\frac{\boldsymbol{r}_{i j} \cdot \boldsymbol{r}_{j k}}{r_{i j} r_{j k}}\right]=\frac{k_{B} T l_{p}}{\sigma}[1-\cos \theta],
$$

where the angle $\theta$ is defined as the angle between consecutive bonds (see Fig. 3.1). Here, $\boldsymbol{r}_{i j} \equiv \boldsymbol{r}_{j}-\boldsymbol{r}_{i}$ is the vector joining two bonded monomers and $l_{p}$ is the persistence length which corresponds to half the Kuhn length $l_{K}$ Doi and Edwards, 1988. For instance, to correctly model hydrated double-stranded DNA (dsDNA) chain embedded in a solvent in physiological conditions, one should take its persistence length to be $l_{p}=l_{K} / 2 \simeq 20 \sigma$, i.e. roughly 20 times its thickness.

A 4-body bonded interaction can be used to capture the torsional stiffness of polymers: The most common choice in this case is a dihedral potential, which can be modelled as a CHARMM MacKerell et al., 1998 potential:

$$
\mathcal{U}_{\text {dihedral }}\left(\boldsymbol{r}_{i}, \boldsymbol{r}_{j}, \boldsymbol{r}_{k}, \boldsymbol{r}_{l}\right)=\kappa_{d}[1+\cos (n \phi-d)]
$$

where $\kappa_{d}$ is the spring energy, $n \geq 0$ is a free parameter, $d$ an integer number of degrees and $\phi$ is defined as the angle between the planes defined by the triplets of atoms $i j k$ and $j k l$ (Fig. 3.1). The angle $\phi$ is defined as $\cos \phi=\hat{\boldsymbol{n}}_{i j k} \cdot \hat{\boldsymbol{n}}_{j k l}$ where $\boldsymbol{n}_{i j k}=\boldsymbol{r}_{i j} \times \boldsymbol{r}_{i k}$ and $\hat{\boldsymbol{n}}=\boldsymbol{n} / n$ is the unit vector. This model is particularly used when studying torsionally constrained polymers, e.g. non-nicked dsDNA, and its 

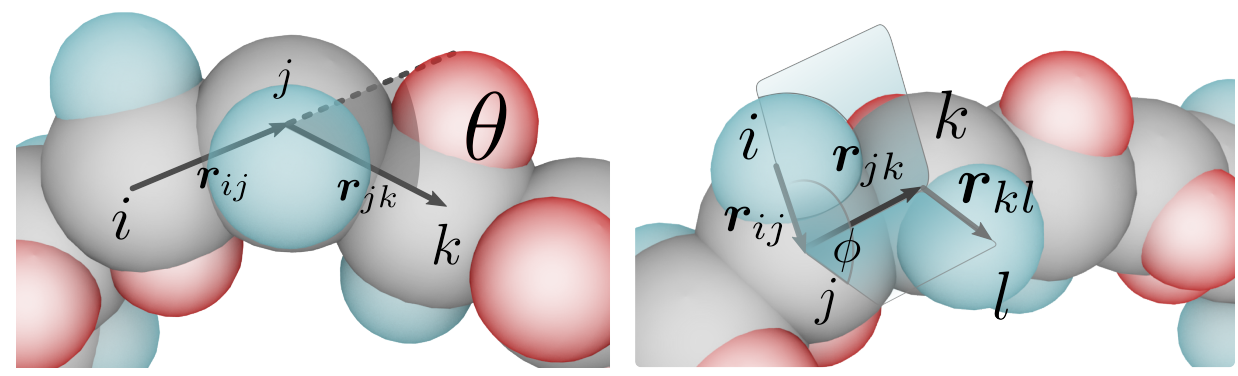

Figure 3.1: The angle $\theta$ for the bending potential in eq. (3.7) is defined as the angle between the vectors joining consecutive pairs along the polymer contour. The angle $\phi$ for the dihedral potential in eq. (3.8) is defined as the angle between the planes defined by the pairs of vectors $\boldsymbol{r}_{i j}, \boldsymbol{r}_{j k}$ and $\boldsymbol{r}_{j k}, \boldsymbol{r}_{k l}$ connecting the polymer backbone (grey beads) to the patches to the sides (blue or red).

consequent supercoiling properties Brackley et al., 2014.

\subsubsection{Brownian Dynamics}

When the subject of the "computer experiment" involves a solvent, and the hydrodynamic interactions can safely be neglected, it is common practice to model the solvent "implicitly". Instead of integrating the deterministic motion of the small solvent particles, one can couple the beads forming the solute with a bath at fixed temperature $T$ : this implies that every atom in the system undergoes some motion which is no longer deterministic but includes a stochastic term. This is modelled as a force that represents the random (frequent) collision of the solute with the solvent (much smaller) particles. This method of simulating systems where the solute molecules are much heavier than the solvent ones is often referred to as "Brownian Dynamics". The equation describing the motion of the atoms is the Langevin equation:

$$
m_{i} \frac{\partial^{2} \boldsymbol{r}_{i}}{\partial t^{2}}=-\xi_{i} \frac{\partial \boldsymbol{r}_{i}}{\partial t}-\frac{\partial \mathcal{U}}{\partial \boldsymbol{r}}+\sqrt{2 k_{B} T \xi_{i}} \boldsymbol{f}_{i}
$$

where $\boldsymbol{f}_{i}$ is a delta correlated white noise with zero mean

$$
\left\langle f_{i}^{\alpha}(t) f_{j}^{\beta}(s)\right\rangle=\delta(t-s) \delta_{i j} \delta_{\alpha \beta}
$$

along each Cartesian component (Greek letters) and $\xi_{i}$ is the friction coefficient of the $i$-th atom. In the limit in which the term on the left hand side of eq. (3.9) is neglected, i.e. in the long-time limit $t \gg m_{i} / \xi_{i}$, the Langevin equation takes the "over-damped" form:

$$
\frac{\partial \boldsymbol{r}_{i}}{\partial t}=-\frac{1}{\xi_{i}} \frac{\partial \mathcal{U}}{\partial \boldsymbol{r}_{i}}+\sqrt{2 D_{i}} \boldsymbol{f}_{i}
$$

where the diffusion coefficient of the $i$-th atom is $D_{i}=k_{B} T / \xi_{i}$. Eq. (3.11) is a good approximation of the motion of atoms described by eq. (3.9) only after an inertial time $\tau_{i n}=m_{i} / \xi_{i}$. The friction $\xi_{i}$ can be expressed as $\xi_{i}=m_{i} / \gamma_{d}$, where $\gamma_{d}$ (having time units) is a damping factor that regulates the time-scale related to temperature 
relaxation. The time-scale $\tau_{\text {in }}$ can therefore be understood as the time required for the temperature to equilibrate across the system, or for the kinetic energy to be redistributed. The parameter $\gamma_{d}$ can be thought of as inversely proportional to the solution viscosity and is directly tuned by the user when integrating eq. (3.9) in the LAMMPS (http://lammps.sandia.gov) engine, which I will employ below.

\section{System Units}

When performing Molecular Dynamics simulations it is often useful to express the properties of the system as multiples of unit-less fundamental quantities. All the results reported in this Thesis from MD simulations will be given in such units. In particular, distances will be measured in units of $\sigma$, masses in units of the monomer mass $m$, time in units of the Lennard-Jones (LJ) time $\tau_{L J} \equiv \sigma \sqrt{m / \epsilon}$, energies in units of $\epsilon$, temperatures in terms of $\epsilon / k_{B}$ and forces in units of $\epsilon / \sigma$.

The LJ time can be understood as the time required by a particle of mass $m$ to be repelled to a distance $\sigma$ by a force $\epsilon / \sigma$, and gives the time-scale for collisions between particles interacting via the Lennard-Jones potential in eq. 3.3. The standard convention for Lennard-Jones reduced units sets $m=\sigma=\epsilon=k_{B}=1$, therefore setting also the LJ time $\tau_{L J}=1$. The inertial time is related to this time by the choice of the damping parameter $\gamma_{d}$, which is units of LJ time. In what follows I will always set $\gamma_{d}=\tau_{L J}$ which gives $\tau_{i n}=\tau_{L J}=m / \xi=1$. This implies that the overdamped limit of the Langevin equation becomes a good approximation of eq. (3.9) already after few timesteps.

Finally, the Brownian time $\tau_{b r}=\sigma^{2} / D_{b}$, where $D_{b}=k_{B} T / \xi$ is the diffusion coefficient of a bead and can be interpreted as the time needed for an atom to diffuse its own size. With this choice of parameters, the Brownian time is equal to the LJ time and the inertial time. Summarising, with this choice of parameters, the relevant time-scales for the microscopic motion of the atoms are all set to 1 in LJ reduced units.

Mapping the reduced LJ units to real units can be easily done by fixing the fundamental quantities in the system, i.e. the length-scale $\sigma$ and the temperature $T$. For instance, when the length-scale $\sigma$ reflects the thickness of hydrated dsDNA, it can take values between $2 \mathrm{~nm}$ to $15 \mathrm{~nm}$ depending on the concentration of salt, or more generally, positive ions, in solution Rybenkov et al., 1993. Here, I will often use the value of $\sigma=2.5 \mathrm{~nm}$ found near physiological conditions $(\mathrm{NaCl}$ concentration of $0.15 \mathrm{M}$ ). The temperature will be set, as usual, at $T=300 \mathrm{~K}$. By using the Stokes formula for the friction of beads of diameter $\sigma$ in a solution of viscosity $\eta_{\text {sol }}$

$$
\zeta=3 \pi \eta_{\text {sol }} \sigma
$$

together with $m / \xi=\tau_{L J}=\sigma \sqrt{m / \epsilon}$ and $\epsilon=k_{B} T$ (see next section) one can obtain 
an expression for the LJ time independent of the mass:

$$
\tau_{L J}=\frac{3 \pi \eta_{s o l} \sigma^{3}}{k_{B} T}=\tau_{b r}=\tau_{i n}
$$

By using the nominal water viscosity $\eta=1 \mathrm{cP}$ one finally obtains $\tau_{L J} \simeq 36 \mathrm{~ns}=$ $\tau_{i n}=\tau_{b r}$. It is worth stressing that the simulation time in real-life units that can be achieved with this scheme scales as the cubic power of the monomer size $\sigma$. This implies that choosing the right level of detail is of paramount importance. In the case of a dsDNA, modelling the chain as a single chain of thickness $\sigma$, or as two spiralling helices, each of thickness $\sigma$, can dramatically reduce the typical simulation time-scale that can be achieved by a factor of about $5^{3}$.

\section{Time Integration}

The integration is performed in the canonical ensemble, i.e. number $(\mathrm{N})$, volume $(\mathrm{V})$ and temperature $(\mathrm{T})$ are conserved. The temperature is fixed by a Langevin thermostat, which couples the system to a bath at temperature $T$ by regulating the magnitude of the thermal noise via the fluctuation-dissipation relation. Since the focus of this Thesis is on systems where the thermal noise plays an important role, the temperature $T$ is chosen so that the thermal energy $k_{B} T$ equals the LJ interaction energy $\epsilon$ used in eq. 3.3. The numerical integration is performed using the velocity-Verlet algorithm Swope et al., 1982] using a time-step of $\Delta t=0.01 \tau_{L J}$. The Verlet class of algorithms has the advantage of conserving the energy, or volume of phase space, during the time-evolution of the Hamilton equations, as opposite to, for instance, the Euler method, which does not ensure energy conservation Frenkel and Smit, 2001. The standard implementation of the velocity-Verlet scheme requires the storing of information for two time-steps. The "velocity-Verlet" version instead has the advantage of storing the information only for a single time-step. The standard Verlet algorithm in the case of systems in which there is no implicit solvent, i.e. in the case the system evolves according to Eqs. (3.1), can be easily derived by looking at the Taylor expansion of the equations of motion around time $t$ :

$$
\begin{aligned}
& \boldsymbol{r}(t+\Delta t)=\boldsymbol{r}(t)+\boldsymbol{v}(t) \Delta t+\frac{\boldsymbol{a}(t)}{2} \Delta t^{2}+\frac{\boldsymbol{b}(t)}{6} \Delta t^{3}+\mathcal{O}\left(\Delta t^{4}\right) \\
& \boldsymbol{r}(t-\Delta t)=\boldsymbol{r}(t)-\boldsymbol{v}(t) \Delta t+\frac{\boldsymbol{a}(t)}{2} \Delta t^{2}-\frac{\boldsymbol{b}(t)}{6} \Delta t^{3}+\mathcal{O}\left(\Delta t^{4}\right)
\end{aligned}
$$

where $\boldsymbol{b}(t)=\frac{\partial^{3} \boldsymbol{r}(t)}{\partial t^{3}}, \boldsymbol{a}(t)$ the acceleration and $\boldsymbol{v}(t)$ the velocity. By summing these two equations one obtains:

$$
\boldsymbol{r}(t+\Delta t)=2 \boldsymbol{r}(t)-\boldsymbol{r}(t-\Delta t)+\boldsymbol{a}(t) \Delta t^{2}
$$


which is the standard form of the Verlet algorithm. It is important to stress that the positions thereby generated are accurate up to fourth order in $\Delta t$. The expression for the velocities can be obtained using:

$$
\boldsymbol{r}(t+\Delta t)-\boldsymbol{r}(t-\Delta t)=2 \boldsymbol{v}(t) \Delta t+\mathcal{O}\left(\Delta t^{3}\right)
$$

which gives:

$$
\boldsymbol{v}(t)=\frac{\boldsymbol{r}(t+\Delta t)-\boldsymbol{r}(t-\Delta t)}{2 \Delta t}+\mathcal{O}\left(\Delta t^{2}\right)
$$

and is only accurate up to second order.

It is instructive also to look at this algorithm from another point of view: instead of integrating Eqs. (3.1) one could attempt to generate set of coordinates that minimise the (discretised) action:

$$
\mathcal{S}=\sum_{n=n_{\mathrm{in}}}^{n_{\mathrm{fin}}} \Delta t\left[\frac{m}{2}\left(\frac{\boldsymbol{r}_{n+1}-\boldsymbol{r}_{n}}{\Delta t}\right)^{2}-\mathcal{U}_{n}(\{\boldsymbol{r}\})\right]
$$

where $\boldsymbol{r}_{n}$ is the position of the atom at time-step $n \Delta t$ and $\mathcal{U}_{n}(\{\boldsymbol{r}\})$ is the total potential taking into account the position of all the atoms in the system at the same time-step. The action $\mathcal{S}$ is stationary when its derivative with respect to all $\boldsymbol{r}_{n}$ is zero. This implies:

$$
m\left(\frac{2 \boldsymbol{r}_{n}-\boldsymbol{r}_{n+1}-\boldsymbol{r}_{n-1}}{\Delta t}\right)-\Delta t \frac{\partial \mathcal{U}_{n}(\{\boldsymbol{r}\})}{\partial \boldsymbol{r}_{n}}=0
$$

or

$$
\boldsymbol{r}_{n+1}=2 \boldsymbol{r}_{n}-\boldsymbol{r}_{n-1}-\frac{\Delta t^{2}}{m} \frac{\partial \mathcal{U}_{n}(\{\boldsymbol{r}\})}{\partial \boldsymbol{r}_{n}}
$$

which is the Verlet algorithm. This explicitly shows that the trajectory generated by this algorithm "shadows" the true trajectory, i.e. the one that minimises the action in Eq. 3.19, and is exact in the limit $\Delta t \rightarrow 0$.

\subsection{Modelling}

This section describes the main models used to simulate ring polymers and gels in this Thesis. Unless otherwise stated, the parameters used have been defined in the previous Section. Most of the models summarised in this work, focus on polymers whose torsional stiffness is neglected; a good approximation when simulating nicked DNA polymers, i.e. dsDNA segments which have a gap along one of the two strands forming the double-helix. In this case the constraint in the torsion is mechanically released and therefore the torsional stiffness can be neglected. 


\subsubsection{Modelling (Knotted) Ring Polymers}

Ring polymers are, in general, prepared as a string of monomer locations that follow a parametrised curve in $\mathbb{R}^{3}$ (see Fig. 3.2 (a)). The large majority of knot types investigated in this Thesis can in fact be described in terms of simple functions from $S^{1}$ to $\mathbb{R}^{3}$. For example the family of $(p, q)$-torus knots Adams, 1994 can be parametrised as

$$
\begin{aligned}
& x(\phi)=r(\phi) \cos (p \phi) \\
& y(\phi)=r(\phi) \sin (p \phi) \\
& z(\phi)=-\sin (q \phi)
\end{aligned}
$$

where $r(\phi)=\cos (q \phi)+2$ and $\phi \in(0,2 \pi)$. This family of knots contains the unknot $\left(0_{1}\right)$, formed as a $(p, 1)$ - or $(p,-1)$-torus knot, the trefoil knot $\left(3_{1}\right)$ formed as a $(2,-3)$-torus knot (left-handed) or as a $(2,3)$-torus knot (right-handed), the pentafoil $\left(5_{1}\right)$ formed as a $(5,2)$-torus knot, et cetera. The knots belonging to this family are knots that can be drawn on the surface of a torus without self-crossings. In particular, $p$ and $q$ represent the number of turns along the "longitudinal" and the "meridional" " direction of a torus, respectively. These have to be relatively prime to form a knot, otherwise one would obtain a link with two or more components.

The figure of eight knot $\left(4_{1}\right)$ is not part of the torus knot family as it cannot be drawn on the surface of a torus (having genus $G=1$ ) but it can be embedded on the surface of a genus $G=2$ surface, i.e. it is a two-embeddable knot Adams, 1994, and has a simple parametrisation:

$$
\begin{aligned}
& x(\phi)=r(\phi) \cos (3 \phi) \\
& y(\phi)=r(\phi) \sin (3 \phi) \\
& z(\phi)=\sin (4 \phi)
\end{aligned}
$$

where $r(\phi)=2+2 \cos (2 \phi)$. Other types of knots which are not torus knots, such as the $5_{2}$ or $6_{1}$, can be described as Lissajous knots Bogle et al., 1994. They can be parametrised as

$$
\begin{aligned}
& x(\phi)=\cos \left(n_{x} \phi+\psi_{x}\right) \\
& y(\phi)=\cos \left(n_{y} \phi+\psi_{y}\right) \\
& z(\phi)=\cos \left(n_{z} \phi+\psi_{z}\right)
\end{aligned}
$$

where $n_{x}, n_{y}$ and $n_{z}$ are (relatively prime) integers and $\psi_{x}, \psi_{y}$ and $\psi_{z}$ are (real) phases. For instance, the three-half twist knot $\left(5_{2}\right)$ can be obtained by setting $\boldsymbol{n}=\left(n_{x}, n_{y}, n_{z}\right)=(3,2,7)$ and $\boldsymbol{\psi}=\left(\psi_{x}, \psi_{y}, \psi_{z}\right)=(0.7,0.2,0)$ while the Stevedore's

\footnotetext{
*The meridian is the circle bounding a disc inside the torus.
} 

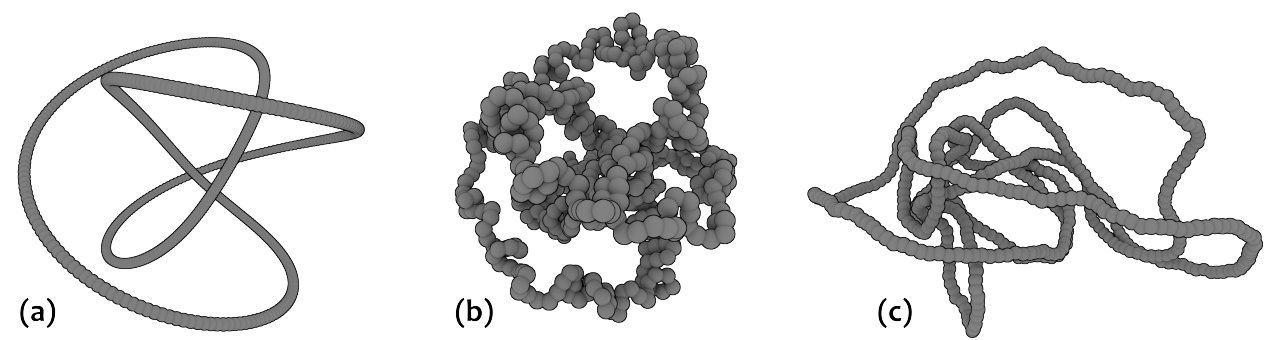

Figure 3.2: Initialising, pre-equilibrating and equilibrating a trefoil knot.

knot $\left(6_{1}\right)$ is found by setting $\boldsymbol{n}=(3,2,5)$ and $\boldsymbol{\psi}=(1.5,0.2,0)$.

Knots for which a parametrisation is not known, such as $6_{2}$ and $6_{3}$, are obtained by simulating polymers which are allowed to self-cross, i.e. $\mathcal{U}_{\mathrm{LJ}}=0$, and selected by computing their Alexander polynomial Orlandini and Whittington, 2007, Micheletti et al., 2011] (see Appendix A for further details on algorithms for identifying knot types). The ones with the desired topology are then considered and used as a template for creating other knotted conformations to be used in other simulations.

\section{Topology-Preserving Equilibration}

Each of the polymers is initialised by performing a short run $\left(\sim 100 \tau_{L J}\right)$ in which the atoms forming a chain $M$ beads long repel each-other via a soft potential and consecutive atoms are connected via a harmonic potential (see Fig 3.2):

$$
\mathcal{U}_{\text {pre-eq }}=\sum_{i=1}^{M}\left[\mathcal{U}_{\text {harm }}\left(\boldsymbol{r}_{i}, \boldsymbol{r}_{i+1}\right)+\mathcal{U}_{\text {bend }}\left(\boldsymbol{r}_{i}, \boldsymbol{r}_{i+1}, \boldsymbol{r}_{i+2}\right)\right]+\sum_{i=1}^{M-1} \sum_{j=i+1}^{M} \mathcal{U}_{\text {soft }}\left(\boldsymbol{r}_{i}, \boldsymbol{r}_{j}\right)
$$

In Eq. (3.24), a modulo- $M$ indexing is taken implicitly, i.e. $M+1 \equiv 1$, in order to join the chain ends. The persistence length is initially taken small $\left(\xi_{p}=1 \sigma\right)$. This allows the system to adjust the length of the bonds and eliminate any unwanted overlapping between atoms at the same time avoiding generating numerical divergences. After the pre-equilibration run, the topology of the knot is generally checked once more, as strand crossings have a non-negligible chance to occur while the soft repulsive potential is on. The equilibration run is then performed using the following intra-chain potential:

$$
\mathcal{U}_{e q}=\sum_{i=1}^{M}\left[\mathcal{U}_{\mathrm{FENE}}\left(\boldsymbol{r}_{i}, \boldsymbol{r}_{i+1}\right)+\mathcal{U}_{\mathrm{bend}}\left(\boldsymbol{r}_{i}, \boldsymbol{r}_{i+1}, \boldsymbol{r}_{i+2}\right)\right]+\sum_{i=1}^{M-1} \sum_{j=i+1}^{M} \mathcal{U}_{\mathrm{LJ}}\left(\boldsymbol{r}_{i}, \boldsymbol{r}_{j}\right)
$$

which strongly suppresses any intra-chain crossings. The length of the equilibration run depends on the system concentration and the ring's length. Typically, this can range between $t_{e q} \simeq 10^{4}$ to $10^{7} \tau_{L J}$ time-steps. In this Thesis I will consider a system "equilibrated" when each of the rings has travelled a mean square distance of at least its own size, i.e. the mean square displacement of the centre of mass of 


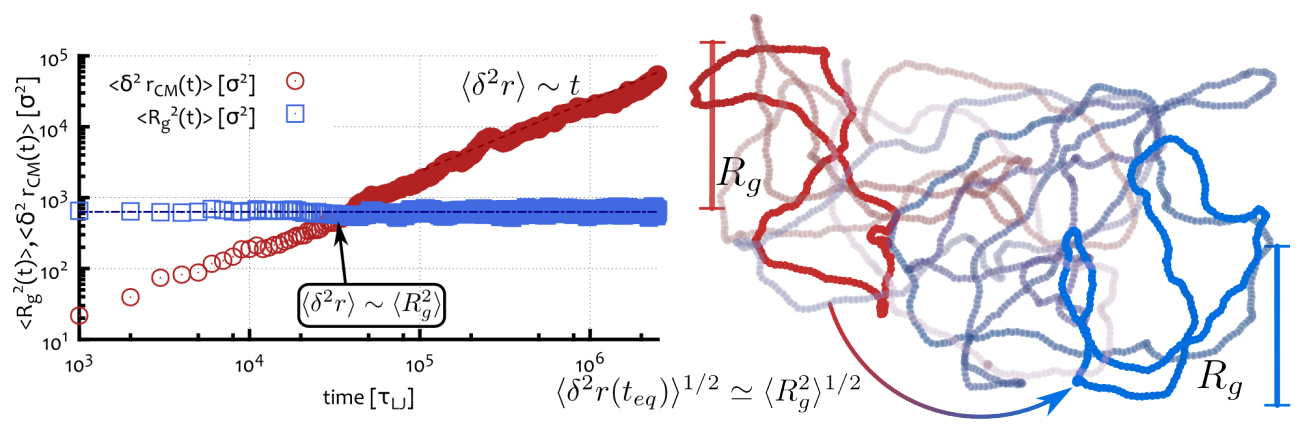

Figure 3.3: Equilibration of a system rings with $M=256$ beads in dilute regime. Red data-points show the mean square displacement of the centre of mass of the rings $\left\langle\delta^{2} r_{C M}(t)\right\rangle$ averaged over the rings. Blue data-points show the average radius of gyration squared $\left\langle R_{g}^{2}(t)\right\rangle$. In the long-time limit the mean square displacement follows the law $\left\langle\delta^{2} r_{C M}(t)\right\rangle=$ $6 D t$, which is here recovered with $D=1 / M$ (see Sec. 3.1.3). The equilibration is reached once each ring has diffused at least once its own size $R_{g}$; in this case $t_{e q} \simeq 310^{4} \tau_{L J}$. On the right, a graphical representation of a ring diffusing its own $R_{g}$ is shown, with red $\rightarrow$ blue a proxy for time.

the rings is at least the radius of gyration squared (see Fig. 3.3).

Throughout pre-equilibration and equilibration runs, the inter-ring interaction can be safely modelled via the Lennard-Jones potential, eq. (3.3), since different rings are normally prepared distant enough to avoid overlapping during the initial time-steps. The inter-chain potential is therefore:

$$
\mathcal{U}_{\text {inter }}=\sum_{\alpha=1}^{N-1} \sum_{\beta=\alpha+1}^{N} \sum_{i=1}^{M} \sum_{j=i}^{M} U_{L J}\left(\boldsymbol{r}_{i_{\alpha}}, \boldsymbol{r}_{j_{\beta}}\right),
$$

where $N$ is the number of chains in the system and the indexes $i$ and $j$ run over the atoms in the chains which are labelled with $\alpha$ and $\beta$. In the case there also is a gel in the system (see Sec. 3.2.2), the interaction between beads forming the polymers (of size $\sigma$ ) and the gel structure (of size $\sigma_{g}$ ) is also modelled as a purely steric interaction via the following potential

$$
\mathcal{U}_{\text {mesh }}=\sum_{k=1}^{M_{g e l}} \sum_{\alpha=1}^{N} \sum_{i=1}^{M} U_{L J}\left(\boldsymbol{r}_{k}, \boldsymbol{r}_{i_{\alpha}}\right),
$$

where $k$ runs over the atoms forming the gel and the minimum distance is $\sigma_{c}=$ $\left(\sigma+\sigma_{g}\right) / 2$.

\section{Characterising Polymer Knots with their Average Crossing Number}

How can we characterise a knot's complexity? This is a very difficult, open problem, which I believe will generate research and scientific discussion for many years to come. Some observables can capture some of the physical properties of knotted polymeric strands. For instance, by parametrising a knot $\mathcal{K}$ as a function $f: S^{1} \rightarrow$ 

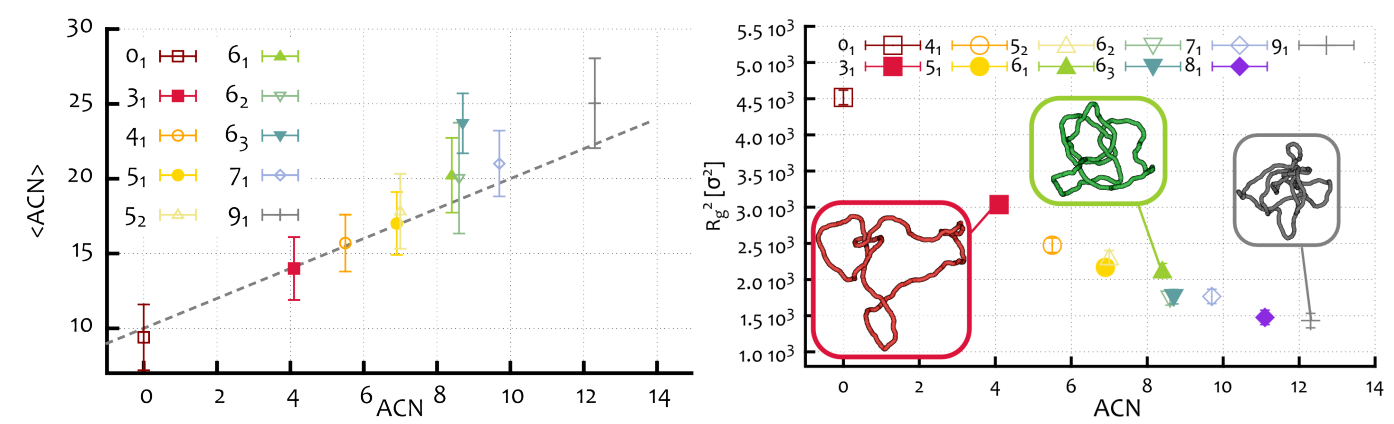

Figure 3.4: (a) Average crossing number averaged over knotted configuration subject to thermal fluctuations $(\langle A C N\rangle)$ as a function of their ACN calculated from energy minimising configurations Kusner and Sullivan, 1994. The one-to-one correspondence allows us to uniquely use the ACN from the ideal configurations. (b) The radius of gyration squared as a function of the ACN clearly shows that more complex knots assume more compact configurations.

$\mathbb{R}^{3}$, one can define the average crossing number $(\mathrm{ACN})$ of $\mathcal{K}$ as its unsigned writhe:

$$
A C N(\mathcal{K})=\frac{1}{4 \pi} \int_{\mathcal{K}} \int_{\mathcal{K}} \frac{\left|\left(f^{\prime}\left(s_{1}\right) \times f^{\prime}\left(s_{2}\right)\right) \cdot\left(f\left(s_{1}\right)-f\left(s_{2}\right)\right)\right|}{\left|f\left(s_{1}\right)-f\left(s_{2}\right)\right|^{3}} d s_{1} d s_{2} .
$$

In some sense, this formula gives the number of crossings of a certain knot configuration, averaged over the 3D space. This has to be compared with the more common "minimal crossing number" (MCN) which is the minimal number of crossings in any knot diagram of a knot (see Appendix A). The ACN is in general higher than the minimal crossing number of a knot and thanks to the "averaging" property of this quantity, the ACN has been found to be an appropriate quantity to characterise the complexity of knots subject to thermal fluctuations and migrating through a medium. In particular, it is worth stressing that the ACN of knotted polymers possesses two crucial properties: (i) the ACN grows with the length of the polymer and it has been found to increase a little faster than linearly for random walks, i.e. $A C N(M) \simeq M \log (M)$ Diao et al., 2003, Orlandini et al., 1994 for a $M$ segments-long walk, and (ii) the ACN of knots can be computed from their ideal configuration, for instance generated as energy minimising configurations Kusner and Sullivan, 1994, Kusner and Sullivan, 1998 or via "tube inflation" procedures Katritch et al., 1996a, and even once these knots are then left to thermalise or their contour length is increased, their thermally averaged (or "mean") ACN is in a oneto-one correspondence to their "ideal" ACN Katritch et al., 1996a]. In this respect, the thermally fluctuating knots retain some of their ideal properties and these are well captured by the ACN Katritch et al., 1996a).

This can be observed in Fig. 3.4(a) where I report the thermally averaged value of the ACN of knots as a function of their "ideal" ACN measured from ideal configurations. In addition, Fig. 3.4(b) clearly shows that more complex knots with same contour length assume more compact configurations. In particular, it was 
found Stasiak et al., 1996 that the square gyration radius is inversely proportional to the ACN, i.e.

$$
\left\langle R_{g}^{2}\right\rangle^{-1} \sim A C N
$$

and that this is related to the faster sedimentation speed Piili et al., 2013, Weber et al., 2013 and faster electrophoretic migration Stasiak et al., 1996 of more complex knots. Because of these reasons, the ACN obtained from ideal configurations is a good quantity for identifying the complexity of knots travelling through media and it will be used in Ch. 6 to uniquely distinguish knot types in that context.

\subsubsection{Modelling a Physical Gel}

Gels are polymer networks formed by cross-linking polymeric strands. Cross-linking is generated by, for instance, the presence of cross-linkers, such as proteins in biological networks Broedersz and MacKintosh, 2014, or a high concentration of polymer bundles, such as in agarose gels [Pernodet et al., 1997], which is one of the most famous and broadly used examples of gels Ross, 1964, Stellwagen, 2009, Viovy, 2000. Agarose gel is commonly used as a buffer to perform gel electrophoresis experiments in order to separate DNA and other bio-polymers. This technique relies on the fact that charged polymeric strands with different lengths, topology or molecular weight possess different mobilities when moving through porous media Calladine et al., 1991, Calladine et al., 1997, Viovy, 2000. The main feature that controls how a gel separates molecules with different sizes is the (typical) mesh size $\xi_{g}$ Calladine et al., 1997. The more peaked the distribution of pore sizes is, the more uniformly the gel acts as a barrier for molecules larger than the pore size. In practice, agarose gels present a broad distribution of pore sizes, which becomes narrower and centred around smaller pore sizes as the concentration of agarose increases Maaloum et al., 1998. In addition, the electrophoretic trapping of circular DNA in agarose gels at strong fields suggests that gels possess dangling fibres which can thread through, and impale, circular DNA and other bio-polymers possessing closed contours Cole and Akerman, 2003. Stellwagen and Stellwagen, 2009.

The Young's modulus of agarose is roughly in the region $E_{\text {agar }}=0.1-1 \mathrm{MPa}$ and depends on the agarose concentration. In any case, it is around 300 times smaller than the one of DNA: $E_{\mathrm{DNA}} \simeq 300 \mathrm{MPa}$ Marko and Cocco, 2003 Kolahi et al., 2012. Nonetheless, the persistence length of single agarose fibre of thickness $\sigma_{\text {agar }} \simeq 2 \mathrm{~nm}$ has been found to be $l_{p}^{\text {agar }} \simeq 10 \mathrm{~nm}$ [Guenet and Rochas, 2006]. Agarose bundles in the sol/gel phase usually form by aggregating a number $n_{f} \simeq 10-20$ of single fibres into "fibrils", which can then display a thickness $\sigma_{\text {fibril }} \simeq 20-40 \mathrm{~nm}$ Pernodet et al., 1997] and much higher persistence lengths. Even by assuming a simple linear scaling of the persistence length of the bundle as a function of $n_{f}$, justified by weak interaction among fibres Mogilner and Rubinstein, 2005], the persistence length of a fibril can still reach values $l_{p}^{\text {bundle }} \simeq 100-200 \mathrm{~nm}$, much higher than than of DNA. 

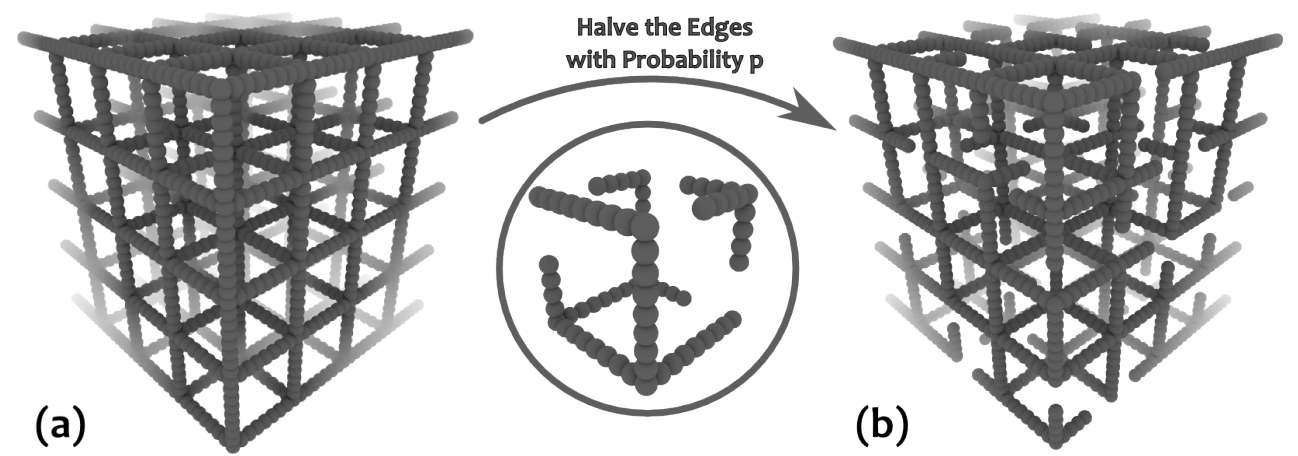

Figure 3.5: Sketch of the gel structure, modelled as (a) perfect rigid cubic lattice and (b) with dangling ends. Modelling a gel as a rigid mesh is particularly appropriate for artificial gels made of solid nano-wires Rahong et al., 2014. Including dangling ends in the model can be of paramount importance when investigating gel electrophoresis of ring polymers (see Ch. 6).

The small Young's modulus of the whole material is often explained as a consequence of the presence of flexible joints in the agarose gel matrix, while the single fibrils and dangling ends Guenet and Rochas, 2006 are generally considered stiff.

Recently developed artificial gels made of solid nano-wires Rahong et al., 2014 provide a more selective pore size distribution, and possess a very large Young's modulus $\left(E_{\text {wires }} \simeq 100 \mathrm{GPa}\right.$ ) which makes the network less susceptible to deformations.

From a theoretical point of view, a gel can be thought of as a collection of strands which create a 3D mesh. For simplicity, this mesh is often approximated as a perfect (cubic) lattice Weber et al., 2006b], with a lattice spacing equal to the average mesh size $\left\langle\xi_{g}\right\rangle$. In this case, the pore size distribution is $p\left(\xi_{g}\right)=\delta\left(\xi_{g}-\left\langle\xi_{g}\right\rangle\right)$. In first approximation, the gel structure can also be considered rigid, i.e. completely static, which is a very good approximation in the case of artificially made gels of solid nano-wires.

In Ch. 4, where I will focus on detecting threadings in systems of rings, I will be modelling the gel as a rigid, perfect, cubic mesh (as in Fig. 3.5(a)). In this case, the microscopic structure of the gel is not expected to play a crucial role in the diffusion of rings. In addition, the fact that the gel is perfect eases the formulation of an algorithm that can unambiguously detect the threadings.

In other cases, as in Ch. 6, modelling the gel microscopic structure in detail is important. For instance, the electrophoretic mobility of ring polymers in gel has been found to be strongly dependent on the presence of dangling fibres Mickel et al., 1977, Turmel et al., 1990, Akerman and Cole, 2002, Cole and Akerman, 2003, Stellwagen and Stellwagen, 2009. Because of this, in Ch. 6, where I will focus on the electrophoretic mobility of knotted ring polymers [Trigueros et al., 2001, Arsuaga et al., 2002, the gel will be modelled as an imperfect mesh, by halving some of the edges forming the cubic lattice with a certain probability $p$ (see Fig. 3.5(b)). 
By tuning the probability $p$ one can directly regulate the average number of dangling ends that can be found in the system, although their length will be fixed to half the lattice spacing, i.e. $l_{d e}=\xi_{g} / 2$. Unless otherwise stated, the gel lattice spacing $\xi_{g}$ has been chosen to reproduce the pore size of an agarose gel at $5 \%$ Pernodet et al., 1997] or of an artificial gel after 3 growth cycles [Rahong et al., 2014], i.e. $\xi_{g}=200 \mathrm{~nm}=80 \sigma$ in MD units. While this value is unusually high for a standard experiment of gel electrophoresis, it is closer to the typical pore size found in high resolution gel electrophoresis Stellwagen and Stellwagen, 2009]. The mesh structure is, unless otherwise stated, made up by static beads of size $\sigma_{g}=10 \sigma \simeq 25 \mathrm{~nm}$, which is compatible with the diameter of either agarose bundles (30 $\mathrm{nm}$ Pernodet et al., 1997) and the nano-wires (20 $\mathrm{nm}$ Rahong et al., 2014).

In general, I will be considering gels whose lattice spacing is comparable with the polymer's Kuhn length, this is because, from a physical perspective, lattice spacings much greater than the Kuhn length can leave the gel so sparse that the rings rarely encounter it. Alternatively, for lattice spacings much shorter than the Kuhn length, the simulation includes an increasingly large fraction of passive gel monomers, which tend to increase the volume fraction of the system and hence limit the concentration of rings that can be studied efficiently using numerical schemes. 
It looks as if it was a bride, walking down the isle, while her dress is being pulled back by flower girls whose dresses are also being pulled by flower girls whose dresses are pulled by other flower girls...

A. Y. Grosberg

\section{4 \\ Threading Rings}

\section{Contents}

4.1 Threading of Rings in a Gel . . . . . . . . . 39

4.1.1 Detecting Threadings between Rings . . . . . . . . . . . . 41

4.1.2 Extensive Threading Leads to Extensive Correlations . . . 45

4.1.3 The Emergence of a Spanning Network of Inter-Threaded

Chains . . . . . . . . . . . . . . . . . 50

4.2 Threading of Rings in Dense Solutions . . . . . . . 52

4.2.1 Overlapping Crumpled Globules . . . . . . . . . . . . 52

4.2.2 The Slow Exchange Dynamics of Rings . . . . . . . . . . 56

4.2.3 Inducing a Topological Glass by Randomly Pinning Rings . 58

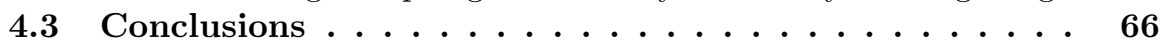

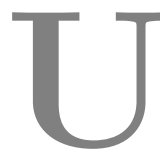

NDERSTANDING the dynamical and rheological properties of solutions of long ring polymers is of primary importance in several areas of soft matter, material science and biophysics Cremer and Cremer, 2001, Kapnistos et al., 2008, Halverson et al., 2011b, Halverson et al., 2013. As mentioned in Ch. 2, ring polymers do not follow the standard reptation theory and in order to make progress it seems that the scientific community will require innovative and unconventional approaches to analyse their properties.

In this Chapter I will address a long-standing problem Klein, 1986, Cates and Deutsch, 1986], that of detecting and investigating threadings between rings in solution. In particular, I will first study ring polymers in a particular ensemble: a solution of ring polymers embedded in a rigid gel. Differently from previous works, where "a background of obstacles" was used to mimic the presence of topological obstacles surrounding any specific ring polymer in solution Rubinstein, 1986, Obukhov and Rubinstein, 1994, Milner and Newhall, 2010, here I intend to introduce a real physical gel, made of a different polymeric units with respect to the ring polymers.

I will focus on this system with the aim of investigating the effect of threadings 
between rings, i.e. inter-chain threading, on the dynamics of the solution and I will show that these are dominant in the limit of large rings, when a spanning cluster of inter-threading rings emerges.

In the second part of this Chapter, I will instead turn my attention to a more conventional system in which ring polymers are forced in a dense solution without the presence of a static gel. In this case, there currently is no algorithm that can unambiguously identify these inter-chain threadings. On the other hand, I will show that these can be detected by appropriately perturbing the system. In particular, this perturbation, borrowed from recent approaches to the glass transition in colloidal and liquid glass formers Berthier and Biroli, 2011, will be shown to trigger a mechanism for which a kinetically trapped state emerges, thereby producing the first in silico evidence of a "topological glass".

In Appendix B, I also summarise some work on the role of "self-threading" events, i.e. entangled states in which a ring polymer threads through itself, in the dynamics of sparse ring polymers embedded in a gel. Because these were investigated by means of a Kinetic Monte-Carlo model, rather different from the MD scheme used in the rest of this Thesis, I found it more appropriate not to include it in the main body of the text.

\subsection{Threading of Rings in a Gel}

The polymers are here modelled as $N$ bead-spring chains each formed by $M$ beads and the dynamics is integrated using the MD scheme as described in Ch. 3. The polymers are prepared unlinked and unknotted from either themselves and the gel (see Fig. 4.1(a)), which is formed by a perfect cubic lattice, i.e. without dangling ends, or equivalently by setting $p=0$ (see Sec. 3.2.2.

The systems under study have $N=50$ polymers with $M=64, M=128$, $M=256, M=512, M=1024$ and $M=1512$ and the box linear size $L$ is changed in order to keep the monomer density fixed at $\rho=N M /\left(L^{3}-V_{\text {gel }}\right) \simeq 0.14 \sigma^{-3}$. Here the polymers Kuhn length is set to $l_{K}=10 \sigma$, the gel lattice spacing $l=l_{K}$ and the mesh structure is formed by beads of size $\sigma$ equal to that of the polymers (see Ch. 3). The reason for this is twofold: (1) if $l \gg l_{K}$ the system would map onto the classic "melt" picture, a system in which I am not interested in this Chapter; (2) if $l \ll l_{K}$ the rings would appear rigid on the length scales of the gel structure and, in addition, the system would be overcrowded with atoms forming the gel structure rather than the rings, which would not be efficient in terms of computer resources. The systems are all well into the overlapping regime, i.e. where coils overlap onto each other. The overlapping monomer density for the system with shortest chains is $\rho^{*}=N^{*} M /\left(L^{3}-V_{\text {gel }}\right) \simeq 0.05 \sigma^{-3}$ where $N^{*}$ is such that $\phi=4 N^{*} \pi R_{g}^{3} / 3\left(L^{3}-V_{\text {gel }}\right)=$ 1. In Fig. 4.1(a) I sketch the system for the case of shortest rings $M=256$ beads.

Even though these systems are not technically a melt of rings, but a dense 


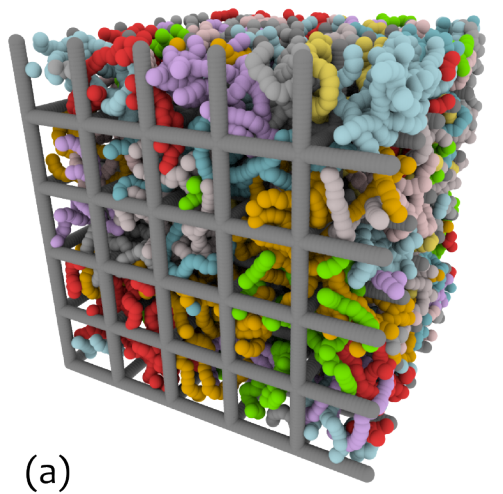

(a)

Figure 4.1: (a) Snapshot of a system with $N=50$ chains of length $M=256$. The gel lattice (grey) can be seen to be filled with ring polymers each shown in different colour. Periodic boundary conditions are used to ensure the absence of surface effects or dangling ends. (b) Segmental mean squared displacement of the rings $\left\langle\delta r_{s}^{2}\right\rangle$ scaled by $\left\langle R_{g}^{2}(M)\right\rangle$ and plotted against time $\tau_{L J}$. Notice the difference from the reptation theory prediction for linear polymers in a melt, where $\left\langle\delta r_{s}^{2}\right\rangle \sim t^{\alpha}$, and $\alpha=1 / 2,1 / 4,1 / 2$ before the tube is renewed and the polymer can freely $(\alpha=1)$ diffuse. The inset shows the scaling of the average squared gyration radius $\left\langle R_{g}^{2}\right\rangle$ as a function of the chains' length. Notice the crossover from "Gaussian" ( $\nu=1 / 2)$ behaviour to crumpled $(\nu=1 / 3)$ globule via the Cates-Deutsch exponent $\nu=2 / 5$ (see Ch. 2).

solution of rings embedded in a gel whose chemical structure could, in principle, differ from that of the polymers, it is tempting to conjecture that rings may not "feel" the difference between topological interaction caused by neighbouring rings and those caused by the gel structure. In light of this, one could argue that the results I will present in this Chapter could, in principle, be translated and applied to the case of a pure melt of rings. In support of this conjecture, in the inset of Fig. 4.1(b), I show the three distinct regimes of the squared radius of gyration $R_{g}^{2}$, i.e. $R_{g}^{2} \sim M^{2 \nu}$ with $\nu=1 / 2 \rightarrow 2 / 5 \rightarrow 1 / 3$, observed in the case of a pure melt of rings Cates and Deutsch, 1986, Grosberg et al., 1993, Grosberg, 2014, Halverson et al., 2011a.

As described in Ch. 2, rings in the melt with $M<M_{e}$, where $M_{e}$ is the rings' entanglement length, are thought to behave similarly to isolated self-avoiding rings in a gef, for which the Parisi-Sourlas result Parisi and Sourlas, 1981 holds and the rings are found to be in the same universality class of self-avoiding annealed branched polymers, with fractal dimension $d_{F}=1 / \nu=2$. For longer rings, the entropy loss due to the conservation of topology, i.e. un-knotted and un-linked from their neighbours, causes the rings to collapse and crossover to a crumpled globule regime, with fractal dimension $d_{F}=3$. Although it is far from being a definite proof that the system considered here can be compared to a melt of rings, it is somehow encouraging that the same scaling of $R_{g}$ is observed here.

*It is interesting to notice that in the ensemble I study in this Chapter, the picture of "rings in a gel", or "in a background of obstacles", is more appropriate than in the case of a pure melt. 
In Fig. 4.1(b) I also report the segmental mean square displacement of the rings, $\left\langle\delta^{2} r_{s}(t)\right\rangle$, defined as

$$
\left\langle\delta r_{s}^{2}(t)\right\rangle \equiv g_{1}(t)=\left\langle\frac{1}{M} \sum_{i}^{M}\left[\boldsymbol{r}_{i}\left(t+t_{0}\right)-\boldsymbol{r}_{i}\left(t_{0}\right)\right]^{2}\right\rangle .
$$

This quantity keeps track of the diffusion of single monomers forming the chains and is therefore very susceptible to any kind of entanglement at the level of segmental relaxation. This shows that for the systems with $M \leq 1024$, after an initial subdiffusive regime where $\left\langle\delta r_{s}^{2}\right\rangle \sim t^{1 / 2}$, the chains freely diffuse only once the polymers have travelled many times their own size, i.e. $\left\langle\delta r_{s}^{2}\right\rangle \geq R_{g}^{2}$. This is similar to the behaviour observed in a melt of rings Halverson et al., 2011b and rather different from the case of a melt of linear polymers Kremer and Grest, 1990 where the free diffusion is reached, via a sequence of distinct sub-diffusive regimes, when the segmental displacement has travelled a shorter distance, of order one $R_{g}^{2}$.

For the longest chains presented here, $M=1512$, the freely diffusive regime has not been achieved in the simulation runtime. It is worth stressing that the same number of time-steps had been run for equilibrating the system and it was considered equilibrated based on the following points Halverson et al., 2011a: (i) the value of $\left\langle R_{g}(t)\right\rangle$ had been observed to settle at a constant value for several decades and (ii) the rings had travelled, on average, at least one $R_{g}$. It is also worth noticing that this system displays a slowing down at large times: After an initial Rouse regime, an entangled regime with $\left\langle\delta r_{s}^{2}\right\rangle \sim t^{1 / 4}$ is observed even at times as large as $410^{7}$ $\tau_{L J}$ timesteps. All this is, once again, compatible with previous findings in the melt Halverson et al., 2011b, which suggests that these two systems are, after all, not so different.

Threading between polymers have been thought of as candidates to drive a dynamical slowing down in systems of rings [Lo and Turner, 2013], and the severe sub-diffusive regime in the segmental displacement (Fig. 4.1) has not yet been investigated in its own right either in a melt or in a gel. In addition, no method to detect topological interactions, such as threadings, exist currently in the melt. This strongly encourages the study of threadings and their effect on the rings dynamics in the system I proposed; as I will show in what follows, the presence of a rigid background structure in fact allows us to unambiguously define and detect the presence of these topological interactions and their persistence in the system.

\subsubsection{Detecting Threadings between Rings}

Threadings are not topological invariants, i.e. a threaded configuration such as that in Fig. 1.2(b) where the shark is threading the other animal, can become un-threaded by smoothly deforming the polymers. For this reason, threadings are very hard to define, let alone identify. 


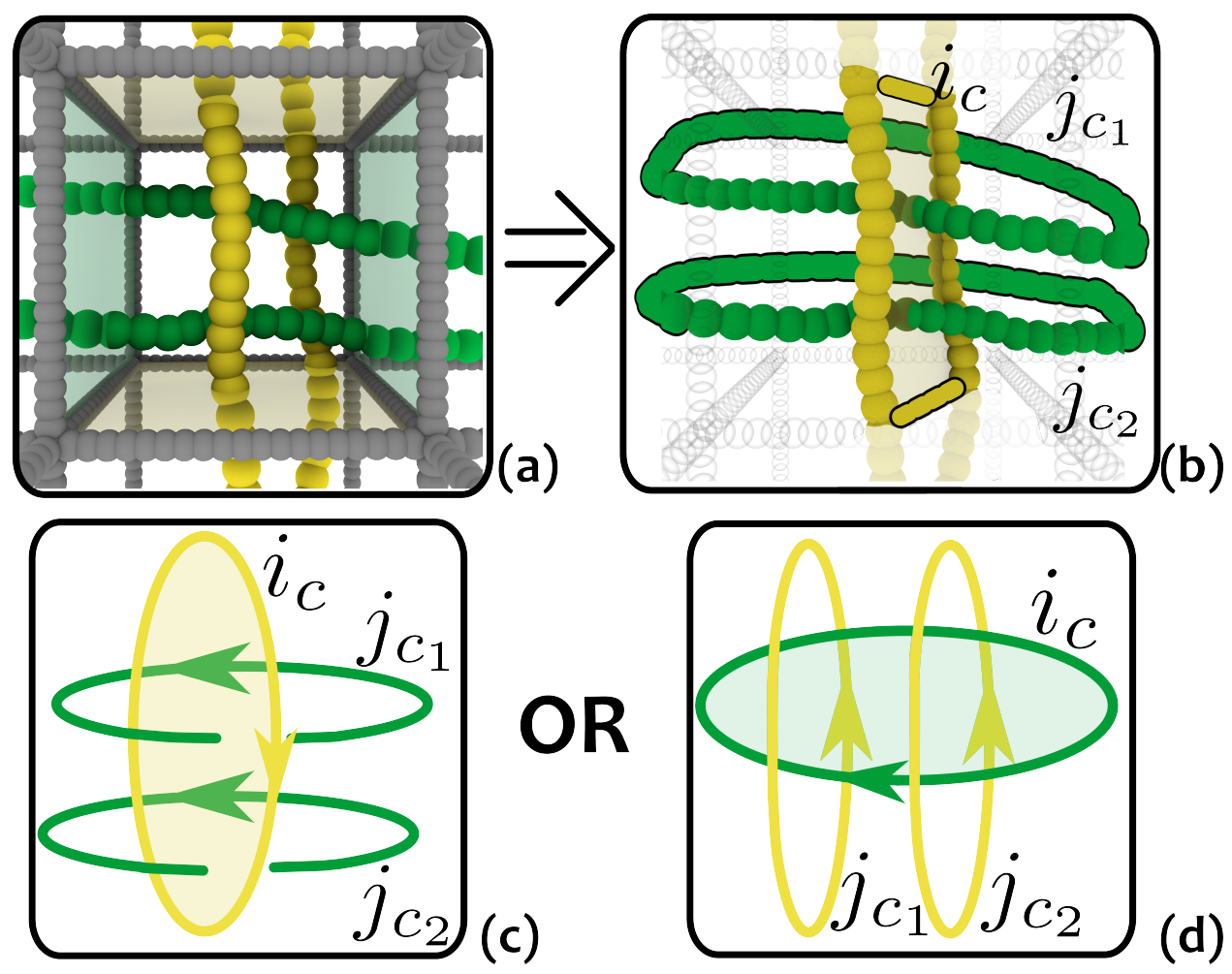

Figure 4.2: Threading identification procedure. The green strands of chain $j$ passing through a face of the cell are separately closed to form two loops $j_{c_{1}}$ and $j_{c_{2}}$. Each one of them is topologically linked with the $i_{c}$, a unique contraction of chain $i$ formed by connecting the points that pass through the faces of the unit cell. In contrast, the green ring is not threaded by the vellow, as one can see by reversing the roles of chains $i$ and $j$ (see $(\mathbf{d})$ ). This implies that threadings can be sub-divided into "passive" and "active" (see later in the text), differently from other types of topological interactions, such as linking, which is mutual.

Threadings can be thought of as local properties of the conformation of the rings; the global topology of the rings remains unlinked from both other rings and the gel. The gel architecture provides a natural local volume - a single unit cell - within which a threading of one ring through another can be identified. This is done by exploiting the following property: each polymer enters and exits a given cell through its faces; the unique topological characteristic of ring polymers, unlinked from the cubic lattice, is that each time the contour passes out through a face of any given unit cell, labelled $c$, this must be accompanied by a returning passage back through the same cell face.

The threading of polymer $i$ by polymer $j$ within cell $c$ can then be defined by performing some operations onto the polymers contour length: First a contraction of ring $i$ is formed by sequentially connecting the points where it passes through any face of cell $c$ by straight lines, as illustrated by the yellow lines in Fig. 4.2(b). This procedure creates a closed loop $i_{c}$ contained entirely within cell $c$ and its bounding faces, and it also takes into account possible re-entrant configurations, where a ring pierces more than once a cell face. This "contraction procedure" in fact joins 
consecutive intersection points of the polymer with the faces of the box in order to form a closed contour entirely contained within one unit cell. In this way the gel is used to identify threadings as local configurations in which the conformation of the ring outside of the chosen cell is unimportant. Next, each of the other strands occupying cell $c$ and labelled by $j_{c}$, belonging to a different polymer $j$, are considered. These strands connect a single entry and exit point through the faces of $c$. The ends of each strand can be closed outside the cell to form a closed loop The next step is then to compute the linking number between each pair of $j_{c}$ and $i_{c}$ thereby created. This will be non-zero if, and only if, ring $i$ is threaded by that strand of ring $j$. There are some cases in which the linking number computation can return zero although the two rings are physically inseparable, as in the case of a Whitehead link Adams, 1994. I expect these exceptions to be rare in our system, as the rings are stiff on the length scale of a gel lattice spacing and therefore such complex configurations are not common.

This procedure is reminiscent of a method used in the literature to measure entanglements in a melt of linear polymers Orlandini and Whittington, 2004, where the authors probed the level of entanglement in the system by ideally carving a box from it and by computing the linking number between pair of curves generated by randomly closing the strands entering and exiting that box. In that case, the procedure was prone to ambiguities in the closing procedure, and, in addition, the size of the box sampled from the system was somehow arbitrary. In the system presented here both these problems are resolved by using the natural entanglement length of the system, i.e. one gel lattice size, this in fact not only gives a natural choice for the size of the probing box but also ensures that every ring entering a face of the box is also exiting the same face, therefore creating a natural choice also for the closing procedurf

To better understand the algorithm, it is perhaps useful to concentrate on a specific example. For instance, consider Fig. 4.2. The two strands of the green ring in Fig. 4.2 (a) are threading the yellow ring. After performing the closing procedure (Fig. $4.2(\mathrm{~b})$ ), the absolute value of the linking numbers between the closed yellow loop and each of the green loops is equal to one (Fig. 4.2(c)). Inverting the procedure by swapping the roles of $i$ and $j$ would lead to the configuration in Fig. 4.2(d), which has linking number of zero between any pair of loops. Note also the emphasis on the fact that I compute the magnitude of the linking number between each pair: the sum of the signed linking numbers would in fact always give zero, as the two original rings are topologically unlinked.

The local threading of ring $i$ by ring $j$ in cell $c$ at time $t$ can therefore be defined by $T h_{c}(i, j ; t)=\frac{1}{2} \sum_{j_{c}}\left|L k\left(i_{c}, j_{c} ; t\right)\right|$ - equal to 1 for the example shown in Fig. 4.2(b)

\footnotetext{
${ }^{\dagger}$ These could be closed at infinity, but anywhere away from the cell would suffice.

${ }^{\ddagger}$ It is fair to say that the similarity between these two methods became apparent, at least to me, only after we finalised the algorithm to detect threadings.
} 

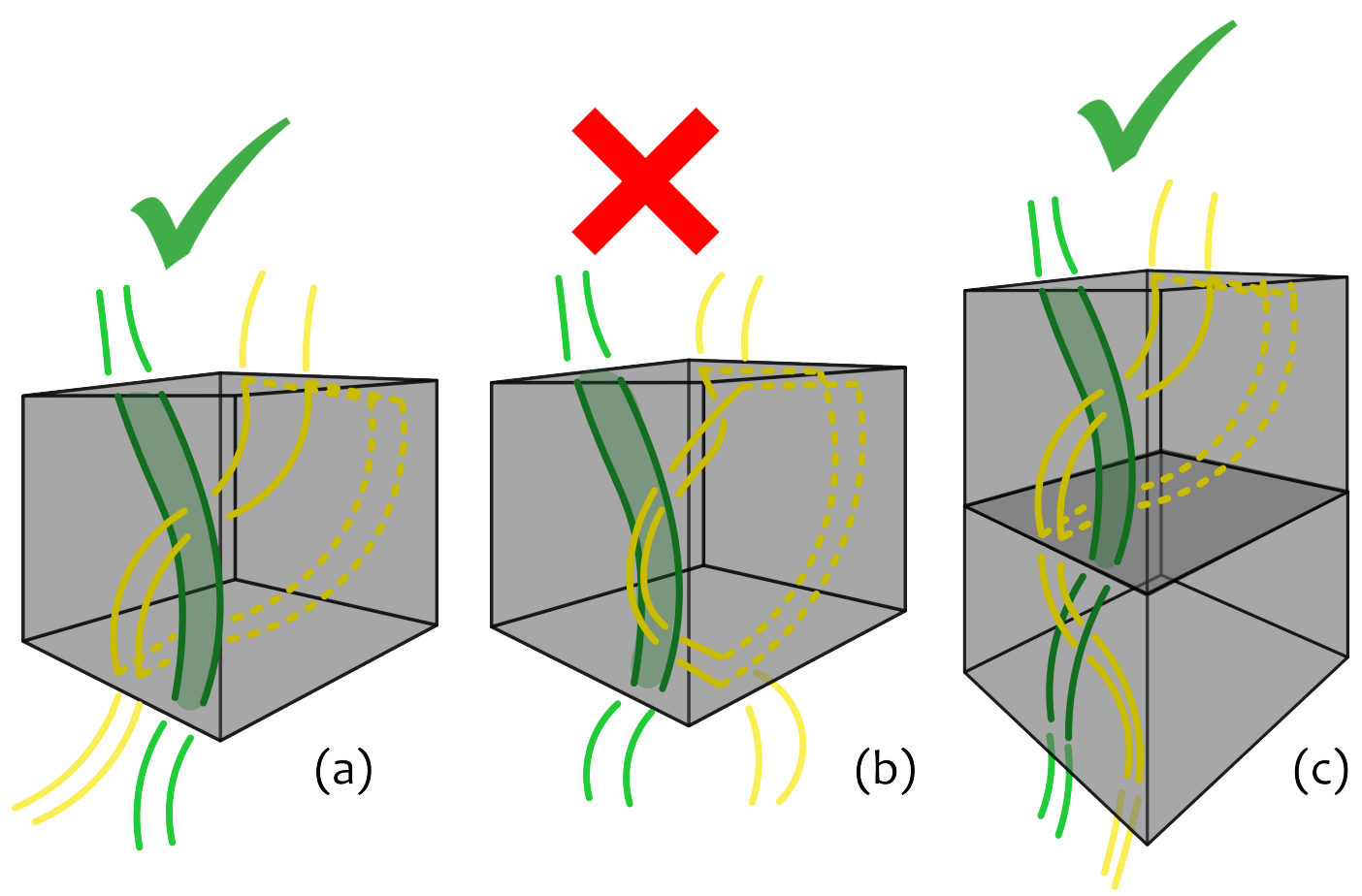

Figure 4.3: Sketches of "tricky" threadings, where rings share the same entry/exit face and detected by the algorithm described in this Chapter. The rings in (a) and (c) would be correctly detected as threading, being the rings configuration locally threaded inside the unit cell. The rings in (b) would also be correctly classified as non-threading being the yellow ring entering and exiting the green contour within the unit cell.

- and the total threadings between these rings by summing this over all cells

$$
T h(i, j ; t)=\frac{1}{2} \sum_{c} \sum_{j_{c}}\left|L k_{c}\left(i_{c}, j_{c} ; t\right)\right| .
$$

This procedure is perfectly well defined, even when identifying threadings associated with rings that share either the entry and/or the exit faces of the unit cell of the gel, as shown in Fig. 4.3. While the definition of threading used in this Chapter is unambiguous, it is helpful to examine what is recorded in the three cases shown: Fig. 4.3(a) The rings thread through each other in the cell. Both of the yellow/yellow-dashed contours, closed at infinity are linked with the green ring, closed by a straight line connecting the points it leaves the cell. The yellow ring is therefore reported as actively threading the green ring by the algorithm. Fig. 4.3(b) There is no threading in the unit cell since the penetrating ring enters, and then leaves, the green shaded area delimited by the green ring contour. The closed yellow/yellow-dashed contours are both unlinked from the green ring, closed on the edge of the box. In this case the algorithm reports three unlinked rings, hence it detects correctly that there is no threading present, hence no topological constraint. Fig. 4.3.(c) Shows the case where there are two local threadings which happen in two different unit cells. In this case, one might argue that by summing the 
contributions of the two cells, the two rings are not threading. On the other hand, in both cells the rings are acting as a constraint for each-other motion within the single cells and therefore the algorithm correctly reports both local threadings. It is finally worth pointing out that pair of threadings like the ones depicted in Fig. 4.3 will usually be short lived, as they can quickly annihilate.

The detection of a threading is necessarily a strictly local measure, on the scale of the cell volume. If the cell volume is increased no threadings will eventually be recorded since rings in the melt are unlinked by construction. A passive threading of ring $i$ by ring $j$ is detected when $T h_{c}(i, j ; t)=1$ and $T h_{c}(j, i ; t)=0$ while the ring $i$ actively threads $j$ when $T h_{c}(i, j ; t)=0$ and $T h_{c}(j, i ; t)=1$. For example, in Fig. 4.2(b), the yellow ring is passively threaded by the green one, and the latter is actively threading the former and not vice versa. This means that threading, unlike linking, is directional and non-mutual.

\subsubsection{Extensive Threading Leads to Extensive Correlations}

The equilibrium average of threadings over rings and over time can be found by taking $\left\langle\sum_{j} T h(i, j ; t)\right\rangle_{i, t} / N \equiv\langle T h\rangle / N$, where $\langle\ldots\rangle_{i, t}$ denotes the average over rings and over time. This represents the number of passive threadings per chain and is reported in Fig. 4.4. As one can notice, this quantity is found to scale extensively with $M$, i.e. it is a critical quantity in the system. Within the lengths used in these simulations, one does not see any deviation from the linear regime. This strongly suggests that longer rings will lead to even more numerous threadings.

One can conjecture that the existence of these penetrations is bound to affect the dynamics of the rings, as they effectively are constraints on the rings diffusion. A measure to quantify the effect of the dynamics is given by the penetrations timecorrelation function

$$
P_{p}(t)=\frac{\left\langle\sum_{j} T h\left(i, j ; t_{0}\right) T h\left(i, j ; t_{0}+t\right)\right\rangle_{i}}{\left\langle\sum_{j} T h\left(i, j ; t_{0}\right) T h\left(i, j ; t_{0}\right)\right\rangle_{i}}
$$

This quantity represents the average fraction of survived passive threadings of ring $i$ after a time interval $t$, see Fig. 4.5(a).

For the longest rings, $P_{p}(t)$ tends to flatten, resembling a plateau, before relaxing to a constant value $P_{p}(t \rightarrow \infty) \equiv P_{p}^{\infty}$, this being the mean probability that two different, randomly chosen chains are penetrating. By using a mean-field argument, one can argue that the probability of threading between two chains in any cell that is occupied by both rings, $p_{t h}$ s can be approximated as the large time probability that they are found threading through one another divided by the number of shared

\footnotetext{
${ }^{\S}$ The same quantity $p_{t h}$ will instead be implemented as a tunable free parameter in the Kinetic Monte-Carlo model described in Appendix B
} 


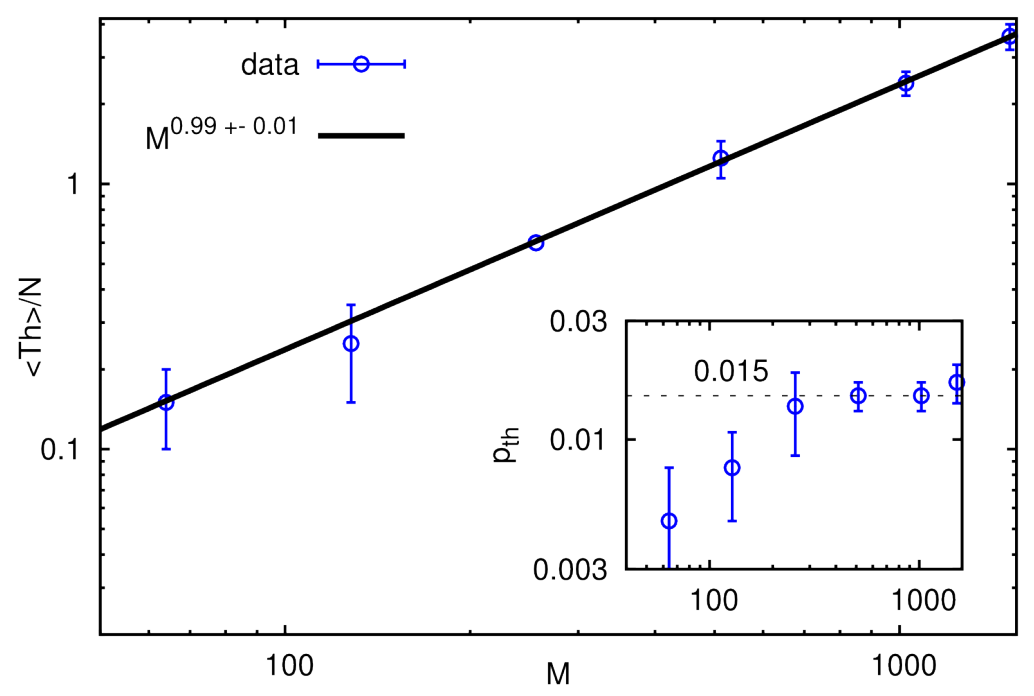

Figure 4.4: Number of threadings $\langle T h\rangle$ per chain as a function of the length of the chains $M$. In the inset we plot $p_{t h}$, crudely the probability of threading in a cell containing two different chains, as computed in eq. 4.4. See text for details.

cells that they both occupy $N_{\mathrm{sc}}$

$$
p_{t h}=P_{p}^{\infty} / N_{\mathrm{sc}} \sim P_{p}^{\infty} \frac{(L / l)^{3}}{M^{2}}
$$

or

$$
P_{p}^{\infty} \sim p_{t h} \frac{M^{2}}{(L / l)^{3}},
$$

where $N_{\text {sc }}$ is the number of cells $c$ shared between two rings each visiting a fraction $f_{c}=N_{v} /(L / l)^{3}$ of the cells in the system, i.e. $N_{\mathrm{sc}} \simeq f_{c}^{2}(L / l)^{3}$. The fraction of cells visited by any one ring was also found to be extensive in the rings length, i.e. $N_{v} \sim M$. This is compatible with the fact that the rings fractal dimension is $d_{F}=3$ in the large $M$ limit, i.e. $R_{g}^{3} \sim M$. Combining this with $P_{p}^{\infty}$ (in Fig. 4.5) one can estimate $p_{t h} \rightarrow 0.015$, reported in the inset of Fig. 4.4. The prediction (dashed line) is particularly well matched by the simulations (data points) at large $M$, where the scaling $R_{g}^{3} \sim M$ is more appropriate to describe the size of the coils. Crudely, this quantity represents the "background probability" of threading between any two ring segments sharing a gel unit cell, and it is independent of any correlations that may have existed at earlier times. The fact that $p_{t h}$ is found to be constant at large $M$ it is very encouraging, as it implies that any two rings sharing a unit cell thread through one another with the same probability $p_{t h}$ independently on their length $M$. As a consequence, by increasing $M$ the system is bound to generate more and more threadings, i.e. constraints on the overall motion of the rings.

In light of this, one could argue that the addition of $\langle T h\rangle$ threadings has the net effect of imposing a number constraints on the system and therefore on the degrees 


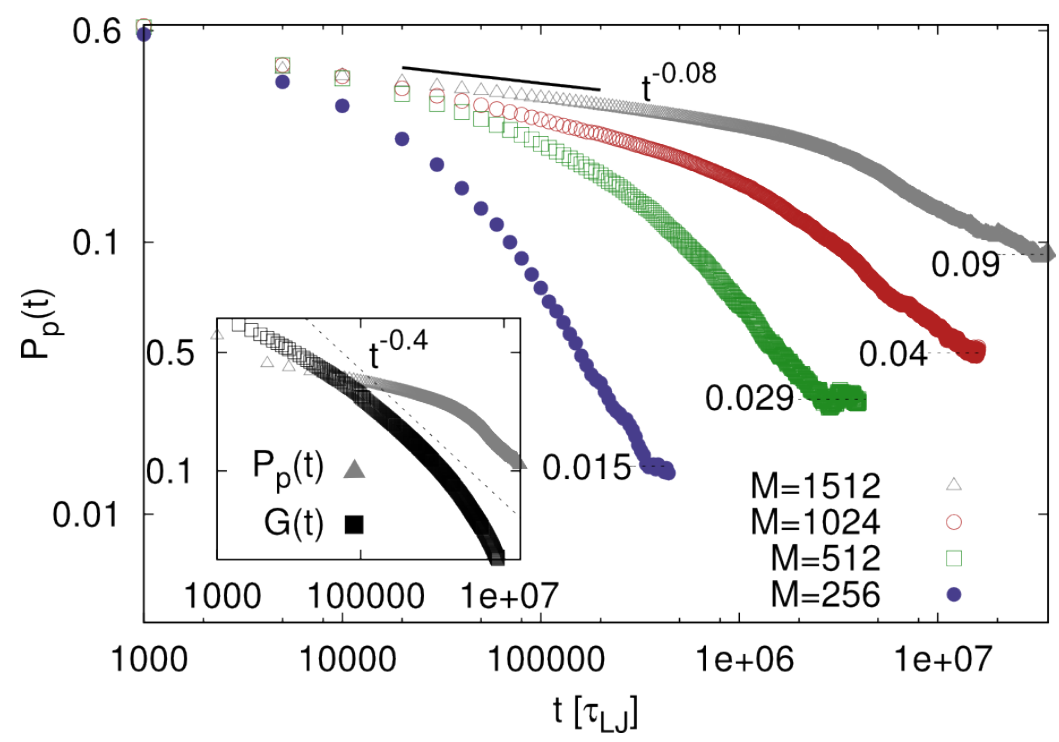

Figure 4.5: Time-correlation function $P_{p}(t)$ of the penetrations (or threading persistence) as computed in eq. (4.3). The inset compares the relaxation of the spatial stress carried by the rings, through the modulus $G(t)$ defined in eq. (4.7), with $P_{p}(t)$ defined in eq. (4.3) for the system with $M=1512$. The spatial stress is clearly relaxed more quickly than the threadings and the dotted line indicates a power law decay of $t^{-0.4}$ Kapnistos et al., 2008.

of freedom, $N_{\text {dof }}$, of the system. Although it might be non-trivial to quantify the degrees of freedom of a melt of rings, it is safe to assume that, because ring polymers have to preserve their topological state, threadings represent a severe (although temporary), constraint on the translational degrees of freedom, $N_{\mathrm{tr}}$, of the chains. As a consequence, one could think that in the case

$$
N_{\text {tr }} /\langle T h\rangle \sim 1,
$$

the system has saturated its translational degrees of freedom, and therefore the chains are all correlated to one another via threadings, hindering the fluid motion of the system. This scenario, that could be (loosely) compared to the Maxwell isostaticity condition of frames Maxwell, 1864, Kane and Lubensky, 2013], captures the primary motivation of this Thesis, i.e. to find a state in which these topological interactions can drive a dynamically arrested state, or a "topological glass". In this respect, a melt of rings is a more promising candidate than, for instance, a blend of rings contaminated with linear chains Halverson et al., 2012. Although such a system has showed a surprising rubbery plateau even after small addition of linear contaminants, there are no topological constraints that can be imposed on linear polymers, and for this reason, the largest relaxation time of the system is bound above by the slowest relaxation of the linear polymers, i.e. $\tau_{\text {relax }} \sim M^{3}$.

In order to better understand the correlation between spatial stress and threading relaxation in the system studied here, one can compare the threading persistence 
$P_{p}(t)$ with the stress relaxation modulus, $G(t)$ Doi and Edwards, 1988. Here, the stress carried by the rings can be computed easily, once again making use of the rigid gel structure, as

$$
G(t)=\frac{\left\langle\sum_{c} g\left(i, c ; t_{0}\right) g\left(i, c ; t_{0}+t\right)\right\rangle_{i}}{\left\langle\sum_{c} g\left(i, c ; t_{0}\right) g\left(i, c ; t_{0}\right)\right\rangle_{i}}
$$

where $g(i, c ; t)=1$ if $\operatorname{ring} i$ is present in cell $c$ at time $t$ and 0 otherwise. While $G(t)$ is a standard quantity in polymer science and usually measures the rheological response, it is likely that, in practice, this quantity would be difficult to separate from that of the gel structure. Tracking the diffusion of labelled tracer rings is therefore likely to be the most effective experimental probe of their dynamics in the system studied here.

From the inset of Fig. 4.5 it is clear that the stress carried by the rings relaxes more quickly than the threadings, i.e. $G(t)$ decays more quickly than $P_{p}(t)$. This is consistent with the fact that one ring, penetrated by another in any particular cell, can independently relax the stress it carries in all other cells. A counter-intuitive finding that has been confirmed several times in the literature Kapnistos et al., 2008, Halverson et al., 2011b, Grosberg, 2014, Halverson et al., 2013, Halverson et al., 2014 , is that rings relax their spatial stress much faster than their linear cousins, $G(t) \sim t^{-2 / 5}$ Kapnistos et al., 2008, leading to a much lower (zero-shear) viscosity

$$
\eta_{0}=\int G(t) d t \sim M^{1.4}
$$

which is compatible with the one observed here (see dotted line in the inset of Fig. 4.5), they have also been observed to continue their sub-diffusive motion long after the stress has completely relaxed. Long-lived penetrations, such as those leading to the long "fat" tails of $P_{p}(t)$ in Fig. 4.5, may be responsible for this remarkable and unique finding. In fact, one could argue that a ring can start its free diffusion $\left(\left\langle\delta r_{s}^{2}\right\rangle \sim t\right)$ only after it has travelled many times its own size $\left\langle R_{g}^{2}\right\rangle^{1 / 2}$ (see Fig. 4.1(b)), i.e. only once the most persistent penetrations have relaxed, on the time-scales shown in Fig. 4.5 .

Fig. 4.6 compares three measures for the relaxation of rings:

(i) the re-orientation time $\tau_{\text {diam }}$, defined as

$$
\tau_{\text {diam }} \equiv \int_{0}^{\infty} C_{\text {diam }}(t) d t=\int_{0}^{\infty} \frac{\left\langle\boldsymbol{d}_{i}\left(t_{0}+t\right) \cdot \boldsymbol{d}_{i}\left(t_{0}\right)\right\rangle_{i}}{\left\langle\boldsymbol{d}_{i}\left(t_{0}\right)^{2}\right\rangle_{i}} d t
$$

where $\boldsymbol{d}_{i}(t)$ is the diameter vector of ring $i$ at time $t$ computed by considering fixed pairs of anti-podal beads along the chain (as in Ref. Halverson et al., 2011a); 
(ii) the overall diffusion time $\tau_{\text {relax }}$ defined as

$$
\tau_{\text {relax }} \equiv\left\langle R_{g}^{2}\right\rangle / 6 D_{C M}
$$

corresponding to the time taken for a chain to diffuse a distance compared to its radius of gyration;

(iii) the un-threading time-scale $\left\langle T_{0.1}\right\rangle$ defined as the solution of the equation

$$
P_{p}\left(T_{0.1}\right) \equiv 0.1
$$

representing the average time required by a ring to lose $90 \%$ of its original threadings, i.e. the time required to "renew" its neighbours $\llbracket$.

Comparing the scaling behaviour of these quantities is exceptionally meaningful: for short rings all these three time-scales are similar to one another, on the other hand, when the size of the rings is increased, one can observed that the internal relaxation decouples from the overall displacement, i.e. $\tau_{\text {diam }} \ll \tau_{\text {relax }}$. This finding is peculiar of ring polymers, since that for linear polymers these two time-scales are related via the tube renewal time-scale. In addition, the un-threading time $\left\langle T_{0.1}\right\rangle$ and the diffusion time $\tau_{\text {relax }}$ seem to instead follow the same scaling, meaning that the time taken to fully diffuse one coil size scales accordingly to the time taken to renew the threadings.

More importantly, these time-scales are observed to deviate from the scaling $\left\langle T_{0.1}\right\rangle \sim \tau_{\text {relax }} \sim M^{2.5}$ when the longest chains are simulated. In fact, for the system with $M=1512$ one finds $\left\langle T_{0.1}\right\rangle \simeq 210^{7} \tau_{L J}$, that is twice as large as the value expected (see Fig. 4.5 and Fig. 4.6), while the value of $\tau_{\text {relax }}$ represents only a lower bound, having not been able to observe free diffusion of these rings within the simulation run-time (and therefore leading to only an upper bound on the value of $D_{C M}$ for the rings with $M=1512$ ). This encourages the conjecture that, at this length, threadings are densely populating the system, thereby creating many long-lived correlations between rings. In particular, one could speculate that the system with $M=1512$ is approaching the regime in which eq. 4.6 holds, i.e. the number of translational degrees of freedom are saturated by inter-chain constraints.

In light of the findings reported in Fig. 4.6, it safe to conclude that the unthreading time-scale is strongly related to the time-scale of the overall ring diffusion and it is perhaps the leading contribution to it. In other words, Fig. 4.5 unambiguously proves that threadings impose inter-chain correlations up to times that can be compared with the time taken by the rings to diffuse their centre of mass over many $R_{g}$.

\footnotetext{
ब $\left\langle T_{0.1}\right\rangle$ can be conceptually compared to the time taken by a linear polymer to renew its confining tube, begin threadings a sort of "tube" (or "cage") for rings.
} 


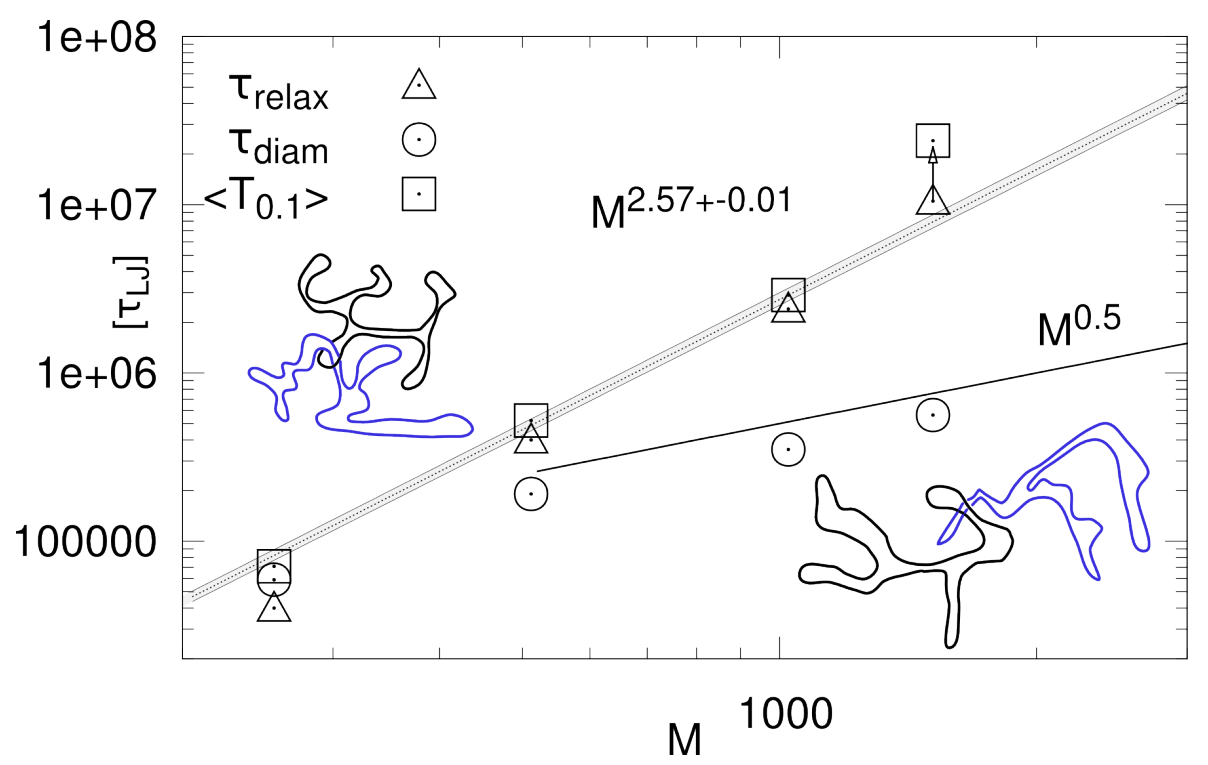

Figure 4.6: Three dynamic relaxation times for rings in concentrated solution. The time taken for internal re-arrangements (captured by $\tau_{\text {diam }}$ ) is shown to be much shorter than $\tau_{\text {relax }}$ and $\left\langle T_{0.1}\right\rangle$, capturing the self-diffusive and the threading relaxation time-scales, respectively (see text for details). The shadowed region delimits the confidence bounds expected for the final data point for the unthreading time $\left\langle T_{0.1}\right\rangle$ at $M=1512$, were it to continue to follow this power law. This point is approximately 30 standard deviations outside the confidence interval, consistent with a dramatic slowing-down due to the development of a strongly connected network of inter-ring penetrations. The arrow on top of the data point showing $\tau_{\text {relax }}$ for the longest rings $M=1512$ indicates that this represents a lower bound: the crossover to diffusive motion has not yet occurred at the longest computationally accessible times. A sketch of two rings fully relaxing their configurations while retaining a threading between them is also show.

\subsubsection{The Emergence of a Spanning Network of Inter-Threaded Chains}

At this stage it is therefore sensible to ask whether it is also possible to spatially relate such topological constraints. In fact, it is reasonable to imagine that, by increasing the length of the rings, one can generate a situation in which every ring is both, actively threading and passively threaded by, a number of other rings. This clearly generates a network of inter-threaded rings. One can therefore ask: are the large-time correlations related to the presence of extended spatial correlations?

In order to quantify the network of penetrations from a "spatial" point of view, it can be useful to make use of the threading matrix $T h(i, j ; t)$ introduced earlier. This keeps track of the threading of ring $i$ by ring $j$ at time $t$ and it is, in some sense, the equivalent of an "adjacency matrix" for the threading rings. The graph representation of the network of threaded rings can therefore be described in terms of a directed graph $G=G(\mathcal{E}, \mathcal{V})$ where $\mathcal{V}$ is the set of vertices, a subset of the

\footnotetext{
"It is interesting to notice that the same question is also frequently asked when studying more conventional glass-forming materials Berthier and Biroli, 2011.
} 


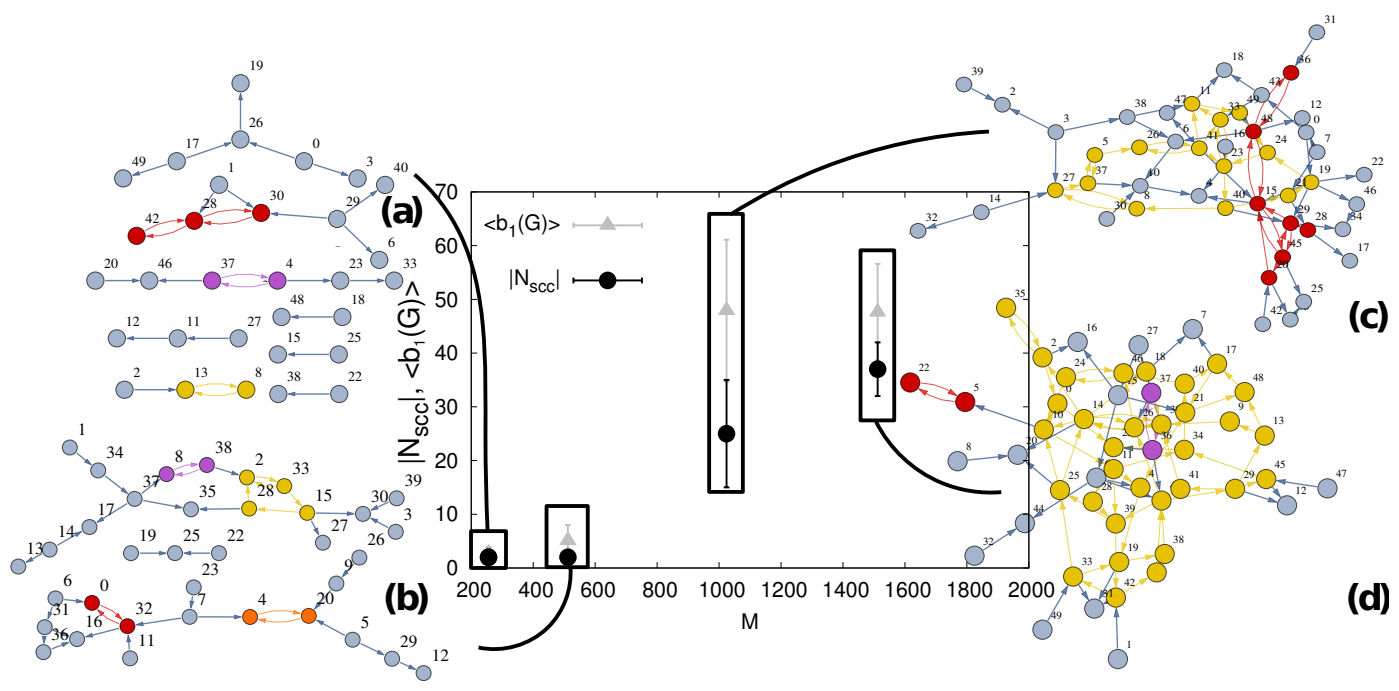

Figure 4.7: Betti number $b_{1}(G)$ and size of the largest strongly connected component $\left|N_{s c c}(G)\right|$ computed by taking the time average of the matrix $T h(i, j ; t)$ after equilibration. Snapshots of some graphs $G(\mathcal{E}, \mathcal{V})$ corresponding to $M=256(\mathrm{a}), 512(\mathrm{~b}), 1024$ (c) and 1512 (d) beads. The colors highlight the strongly connected components in the graphs (see text for details). The numbers near the nodes identify the rings in the simulation. Notice that $N=50$ is the maximum number of rings in the system and the situation $\left|N_{s c c}(G)\right| \simeq 50$ is expected to be reached at around $M=2000$ by extrapolating the last two data points.

set of $N$ rings in the system, and $\mathcal{E}$ is the set of directed edges from ring $j$ to ring $i$, which represent the threadings of $\operatorname{ring} j$ through ring $i$. The time-evolution of the network is given by $T h(i, j ; t)$, and from this it is possible to quantify the emergence of extended structures of inter-threading rings by using the size of the largest strongly connected component $\left|N_{s c c}\right|$ and the first Betti number $b_{1}(G)$, which naively represents the number of 1-dimensional circular holes in the network, and is therefore a signature of "looped" correlated clusters of rings.

Recalling that a strongly connected component is defined as a sub-graph $\left(G_{s c c}\right)$ of $G$ for which every vertex has a path to reach every other vertex within that sub-graph, one can notice that every ring in $G_{s c c}$ has to have at least one passive (in-coming arrow) and one active (out-going arrow) threading. In other words, all the rings belonging to a strongly connected component are related to one another via a chain of threadings.

In Fig. 4.7I report the computed values of $N_{s c c}$ and $b_{1}(G)$ averaged over time and show snapshots of some networks as an example. One can notice that while for short rings the components are small, i.e. the network is fragmented, this is no longer true for longer rings. In this case one observes the formation of larger clusters. When the largest connected component contains $\mathcal{O}(N)$ vertices, it signifies that a percolating cluster of inter-penetrating rings has emerged. At this stage, every ring is connected and correlated with some other ring via a sequence of threadings. While such a representation is static, i.e. if one took two snapshots of the network at different times it would look very similar, it is clear that the emergence of system-spanning 
clusters of threaded rings has to affect the dynamics of the rings. I conjecture that the dynamical effect, or dynamic transition, is actually captured by the significant deviation showed in Fig. 4.6. In other words, the strong increase of relaxation timescales observed in Fig. 4.6 is related to a corresponding increase of spatial correlations which can be captured by the emergence of a system-spanning connected component of inter-threaded rings.

\subsection{Threading of Rings in Dense Solutions}

In the previous Section I introduced a method to detect and identify threadings in a solution of rings embedded in a static gel structure. An obvious extension of this work is to try and quantify the effect of threadings in a dense solution. Clearly, the same protocol that I developed in Sec. 4.1.1 will be no longer valid. In this Section I will therefore first investigate a solution of rings and then attack the problem of characterising threadings from the kinetic point of view by taking inspiration from recent protocols advanced by some authors in the Condensed Matter community working on glass-transitions Biroli et al., 2008, Berthier and Biroli, 2011 Cammarota and Biroli, 2012, Karmakar and Parisi, 2013.

In practice, it is computationally straightforward to transform the system investigated in Sec. 4.1 to a pure solution of rings. By taking equilibrated configurations and deleting the beads forming the gel, similar to an in silico etching, one can in fact obtain pure solutions of $N$ rings $M$ beads long. The monomer density of the systems thereby obtained is lower, i.e. $\rho=0.1 \sigma^{-3}$, and therefore one expects that the rings will accommodate in order to fill the vacant space. Technically, at these densities, the systems are classified "dense solutions" rather than "melts" being only moderately above the overlap (number) density $\rho^{*} \simeq 0.03 \sigma^{-3}$. For computational purposes, I will not increase the density further, hoping that this will not invalidate the extension of the results reported in this Section to the case of concentrations that more closely mimic the melt state. After thermalisation, the systems look like the one sketched in Fig. 4.8(a) where I also report a schematic illustration of a configuration in which many rings thread through one another in a hierarchical fashion starting from the black ring (Fig. 4.8(b)).

\subsubsection{Overlapping Crumpled Globules}

As discussed in Ch. 2, the size of a ring polymer in the melt is that of crumpled globule. In reality, this regime is achieved only when rings are large. Shorter rings assume the ideal size with $\nu=1 / 2$ which crossovers, via $\nu=2 / 5$, to the collapsed regime with $\nu=1 / 3$ (see Fig. 4.9). Although this exponent provides us with information regarding the overall size of the polymer coils, it does not inform us of their internal structure. A better way to probe the internal arrangement of the coils is 
(a)

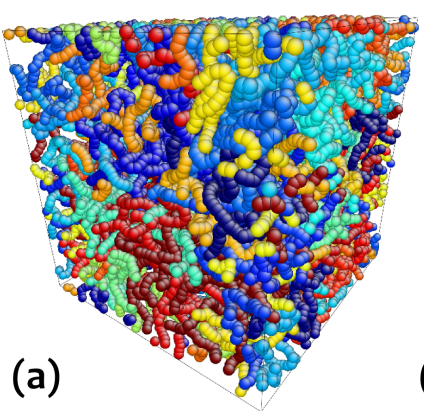

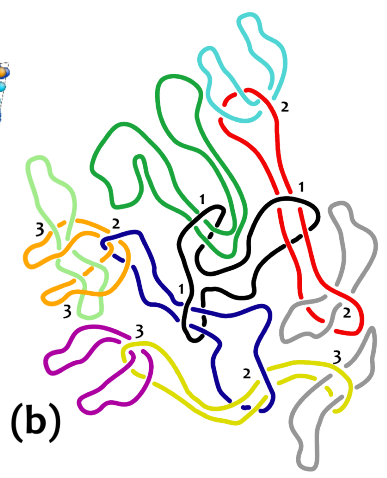

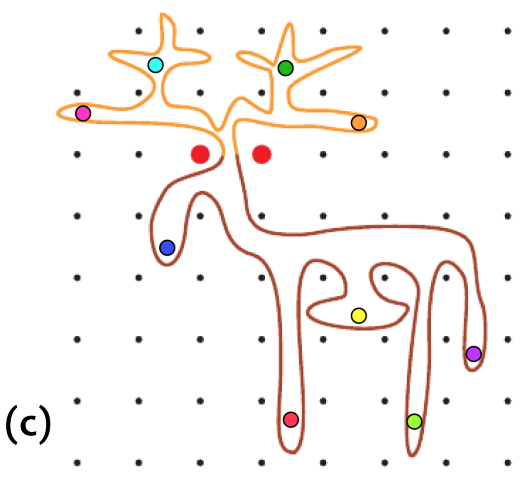

Figure 4.8: (a) Snapshot from MD simulations of a melt with $N=50$ and $M=256$. (b) Schematic representation of inter-threading rings forming hierarchical levels of threadings enumerated from the innermost to the outermost with respect to the black ring. (c) Pierced lattice animal representation of rings in solution (modified from Ref. [Kapnistos et al., 2008]).

by using the static scattering function, or form factor, $S_{1}(q)$ defined in Ch. 2 (see eq. (2.28)). One in fact expects that, in the range $2 \pi / R_{g}<q<2 \pi / \sigma$, this function scales as

$$
S_{1}(q) \sim(q \sigma)^{-d_{F}}
$$

where $d_{F}$ is the fractal dimension of the chain at length scale $2 \pi / q$ and it is related to the entropic exponent as $d_{F}=1 / \nu$ Rubinstein and Colby, 2003]. Linear chains in the melt display $d_{F}=2$ for a broad range of $q$ 's Halverson et al., 2011a. Ring polymers in the melt have instead a more complex arrangement: because their ideal size differs from the size assumed in the limit of large polymerisation index, one can in principle detect two regimes: at contour lengths below $\left(s<M_{e}\right)$ and above $\left(s>M_{e}\right)$ the entanglement length, or blob size, $M_{e}$. In fact, below $M_{e}$ one expects that the chain simply follows the Gaussian statistics for an open polymer $s$ monomers long; for this reason one expects the size of a blob to scale as $R_{b}\left(s<M_{e}\right) \sim s^{1 / 2}$ (being the self-avoidance screened out) Grosberg, 2014. On the other hand, larger contour lengths are more crumpled and follow $R_{b}\left(s>M_{e}\right) \sim s^{1 / 3}$. This markedly different behaviour cannot be captured in melt of linear polymers, as both regimes, below the above the entanglement length, follow the Gaussian statistics with $R_{b}(s) \sim s^{1 / 2}$.

For the rings the situation is more complicated, as represented in Fig. 4.9, Let us focus on the longest rings $M=2048$ : For $q R_{g}>2 \pi R_{g} / l_{K} \simeq 16$, where $l_{K}=10 \sigma$ is the polymers' Kuhn length, the structure factor probes length scales below the Kuhn length and therefore returns, as expected, a scaling $S_{1}(q) \sim q^{-1}$, valid for rigid rods. In the regime, $\pi<q R_{g}<2 \pi R_{g} / l_{K}$, the scattering function probes lengthscales between $2 R_{g}$ and $l_{K}$. In this case, Fig. 4.9 shows that the fractal dimension $d_{F}$ changes from $d_{F}=3$ to $d_{F}=2$ at around $q R_{g} \simeq 4$ corresponding to length scales $l=2 \pi R_{g} / 4 \simeq 40 \sigma$, i.e. several Kuhn lengths but smaller than the diameter of the chain $2 R_{g}$. In the large length scale limit, $q R_{g} \ll 1$, the magnitude of wave vector $|\boldsymbol{q}|$ is much smaller than any distance $\left|\boldsymbol{r}_{i}-\boldsymbol{r}_{j}\right|$ between beads in the chain 


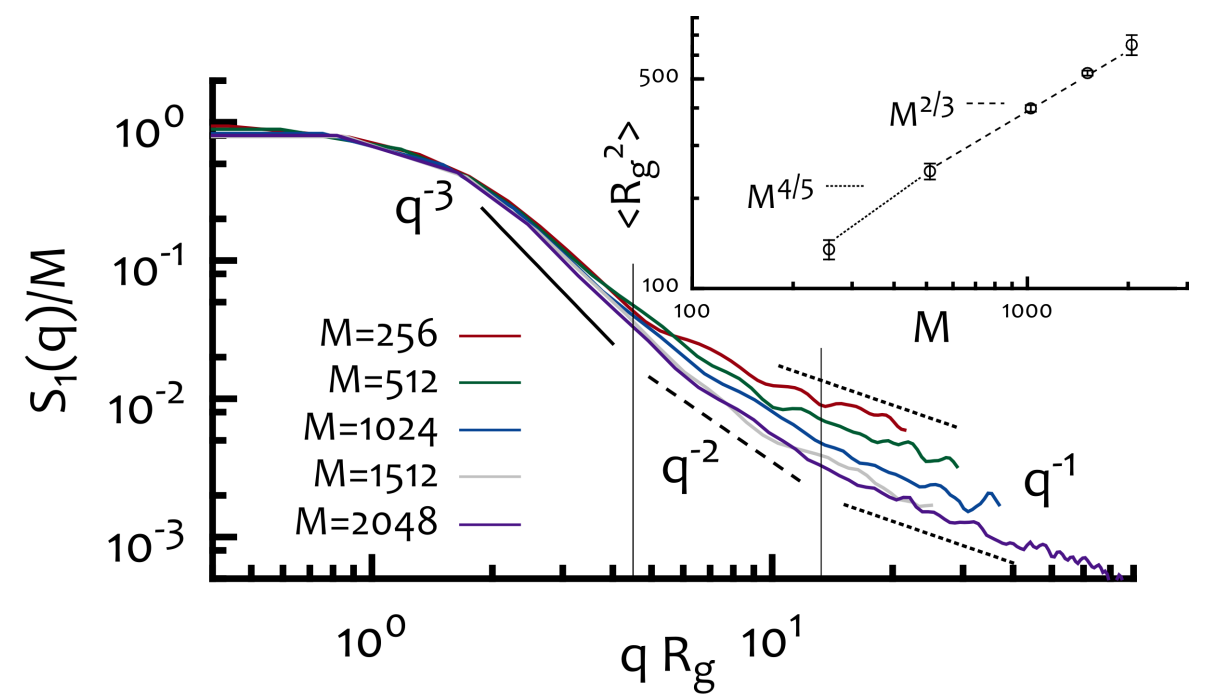

Figure 4.9: Static scattering function $S_{1}(q)$ plotted against $q R_{g}$ and divided by the length $M$ of the rings. The scattering vector $q$ probes length scales $l=2 \pi R_{q} /\left(q R_{g}\right)$ and shows a more complex behaviour than for systems of linear polymers. (Inset) Average radius of gyration squared $\left(\left\langle R_{g}^{2}\right\rangle\right)$ showing the two scaling regimes $R_{g}^{2} \sim M^{2 \nu}$ with $\nu=2 / 5$ and $\nu=1 / 3$ (see Ch. 2).

and therefore the structure factor is simply $S_{1}(q)=M$.

In Fig. 4.10I report the scaling of surface monomers $m_{s}$ computed as the number of monomers of each chain "in contact" with other chains, i.e. having a distance $d \leq \rho^{-1 / 3}$ from other monomers, and $\rho^{-1}$ being the free volume available to each monomer in units of $\sigma$. This quantity has been discussed in Ch. 2 and, in particular, its exponent $\beta$ has been recognised to be a good measure of the coils "roughness". Contact exponents $\beta$ close to one indicate highly rough surfaces with a large number of interactions with other chains. In Fig. 4.10 one can observe that $\beta \simeq 1$ within errors for the systems with the longest chains. It is worth stressing that it might seem counter-intuitive that a globule with exponent $\nu=1 / 3$ and a contact exponent $\gamma \simeq 1$ (reported in Fig. 4.10(c)) has a number of contacts that scales nearly linearly with the length of the coil $M$. The only plausible explanation is that although the coils are collapsed and crumpled, they are also highly inter-penetrating and not segregating. All this strongly suggests that rings in melt might inter-thread, similarly to the rings embedded in the gel studied in the previous section.

The fact that coils are inter-penetrating rather than segregating is also strongly suggested by the pair correlation function of the coils centre of mass:

$$
g(r)=\frac{2}{N(N-1)} \sum_{I}^{N} \sum_{J}^{N} \delta\left(\left|\boldsymbol{r}_{C M, I}-\boldsymbol{r}_{C M, J}\right|-r\right) .
$$

This function shows a distinct peak at $r<2 r_{\max } \simeq 2.6 R_{g}$, being $R_{g}=\sqrt{3 / 5} r_{\max }$ the mean radius of an homogeneous sphere of radius $r_{\max }$, (see Fig. 4.10(d)), and therefore implies that the coils behave similarly to ultra-soft colloids of size $2 r_{\max }$ 

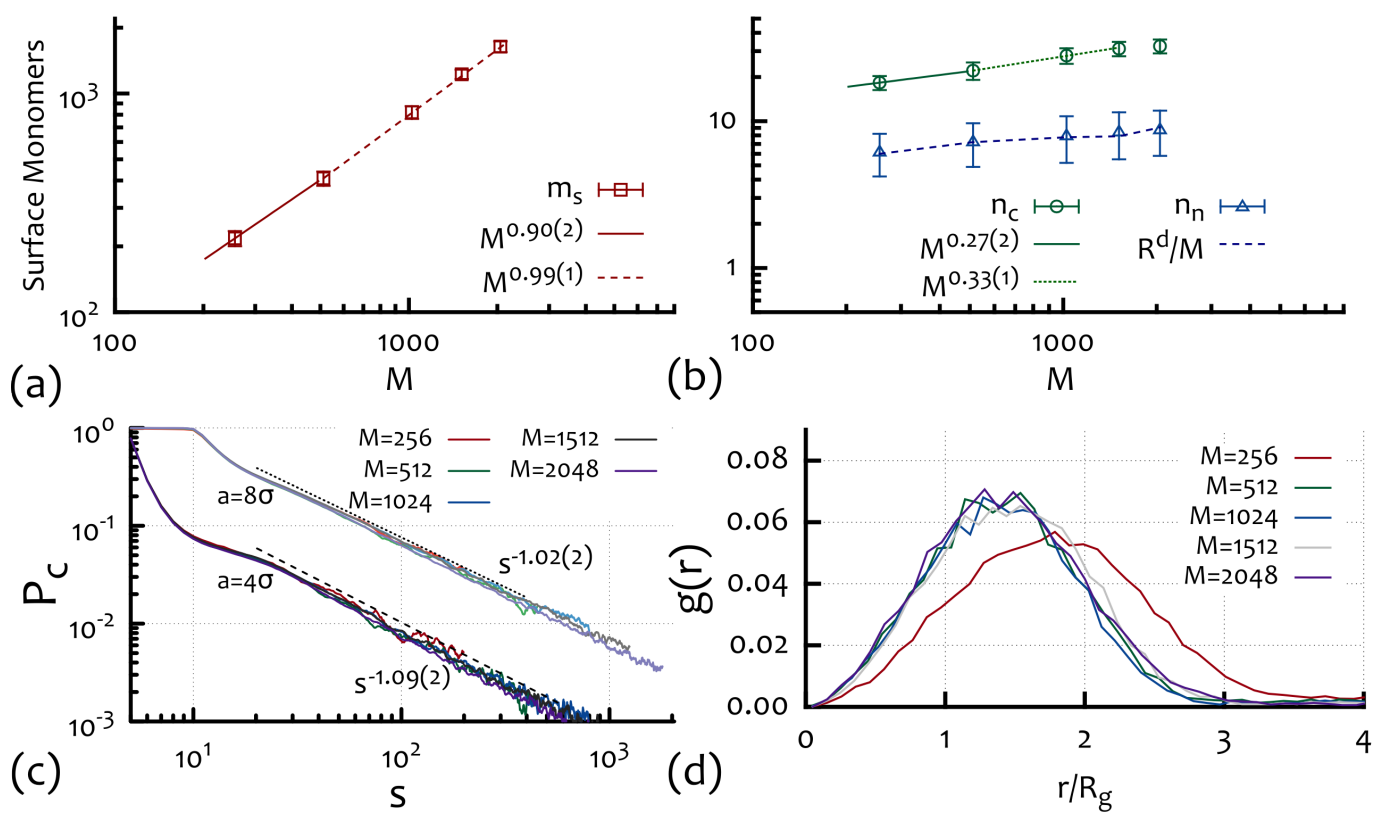

Figure 4.10: (a) Surface monomers $m_{s}$ computed as described in the text and the two observed scaling $m_{s} \sim M^{\beta}$ with $\beta=0.9$ and $\beta=0.99$ in agreement with Ref. Halverson et al., 2011a and Ch. 2. (b) Number of contiguous chains $n_{c}$ and neighbouring chains $n_{n}$ as defined in the text. Notice that the former is quantitatively more numerous than the latter; $N=50$ is the maximum number of chains in the systems. (c) Contact probability $P_{c}(s)$ as defined in Ch. 2 and observed to follow a scaling near $s^{-1}$ as predicted in eq. 2.20 for a crumpled globule (slightly dependent on the chosen cut-off $a$ ). (d) Pair correlation function of the coils centre of mass $g(r)$ as defined in eq. 4.13). The maximum of this function defines the average distance of the chains centre of mass and it is found to be smaller than $2 R_{g}$.

rather than segregated hard spheres Likos et al., 2014]. In light of this, it is reasonable to study the number of different chains with which each coil can interact with. A very crude approximation of the number of neighbours is $n_{n} \sim R_{g}^{d} / M$, that gives the number of chains within a d-dimensional volume of linear size $R_{g}$ centred in any one coil. Equivalently, one can count the number of coils whose centre of mass separation is smaller than sum of their gyration radii, i.e.

$$
n_{n}=\left\langle\sum_{J} \Theta\left(R_{g, I}+R_{g, J}-\left|\boldsymbol{r}_{C M, I}-\boldsymbol{r}_{C M, J}\right|\right)\right\rangle_{I, t},
$$

where $\Theta(x)$ is the usual Heaviside function and $\langle\ldots\rangle_{I, t}$ identifies the average over rings and time-steps. One can imagine to define a different measure of "contact" exploiting the measure of the surface monomers. One can define the number of contiguous chains $n_{c}$ as the number of chains whose monomers are in contact. It is worth noting two features of $n_{c}$ as reported in Fig. 4.10(b): (i) it is larger than $n_{n}$, meaning that the contacts between different chains are not restricted to neighbouring chains within a volume $R_{g}^{d}$ but go beyond the gyration radius, possibly via long 
protrusions; (ii) while $n_{n} \sim R_{g}^{d} / M \sim M^{d \nu-1}$ it is roughly constant $(d \nu-1=0)$ in the crumpled regime, the number of contiguous chains $n_{c}$ seems to increase as $M^{1 / 3}$ before plateauing for the longest chains at around $N \simeq 30$. This means that any given chain is contiguous, in the sense defined earlier, to 30 other chains in the system.

All this is in agreement with previous observations in the melt, where the rings have been found to assume configurations possessing long protrusions and strongly deviating from a compact sphere [Halverson et al., 2011a]. This encourages us to probe the dynamics of the rings, and in particular, to design a protocol that allows us to investigate the effects of threadings and their spatial correlations.

\subsubsection{The Slow Exchange Dynamics of Rings}

At this stage it is reasonable to ask whether one can explicitly measure an interchain correlator in order to unambiguously observe the exchange dynamics of rings in solution. Recently, this has been done by tracking neighbouring chains Lee et al., 2015, i.e. chains whose centres of mass are closer than the sum of their size. Here, we want to exploit the computation of contiguous chains, to define a correlator of contiguity over time. This is done by computing a dynamic $N \times N$ matrix $P(t)$ whose elements are defined as

$$
P_{I J}(t)=\left\{\begin{array}{l}
0 \text { if } d_{i j} \geq \rho^{-1 / 3} \\
1 \text { if } d_{i j}<\rho^{-1 / 3}
\end{array}\right.
$$

where $d_{i j}$ is the distance of any two monomers $i$ and $j$ belonging to chains $I$ and $J$, respectively. From this it is straightforward to obtain the correlator

$$
\varphi_{n c}(t)=\left\langle\frac{1}{N} \sum_{J=1}^{N} P_{I J}(t) P_{I J}(t-\Delta t) \ldots P_{I J}\left(t_{0}\right)\right\rangle_{I, t_{0}}
$$

where $\langle\ldots\rangle_{I, t_{0}}$ indicates the average over rings and initial times $t_{0}$. This function quantifies the exchange dynamics of contiguous chains and, in particular, it is bound to track the quickest time-scale in the exchanging process since it involves the product of $P_{I J}$ over all the intermediate time-steps between $t_{0}$ and $t$. In other words, if two chains are not contiguous at any time during the simulations, that pair no longer contributes to $\varphi_{n c}(t)$, i.e. $\lim _{t \rightarrow \infty} \varphi_{n c}(t)=0$.

Fig. 4.11 shows the behaviour of $\varphi_{n c}(t)$ as a function of the time-lag and for different systems. The exchange dynamics of contiguous pairs can be fitted as a simple exponential for the shortest chains with $M=256$, while for longer chains the behaviour of $\varphi_{n c}(t)$ is best fitted by stretched exponentials of the form

$$
\varphi_{n c}(t)=\exp -\left(\frac{t}{\tau_{\mathrm{nc}}(M)}\right)^{\beta_{\mathrm{nc}}(M)}
$$




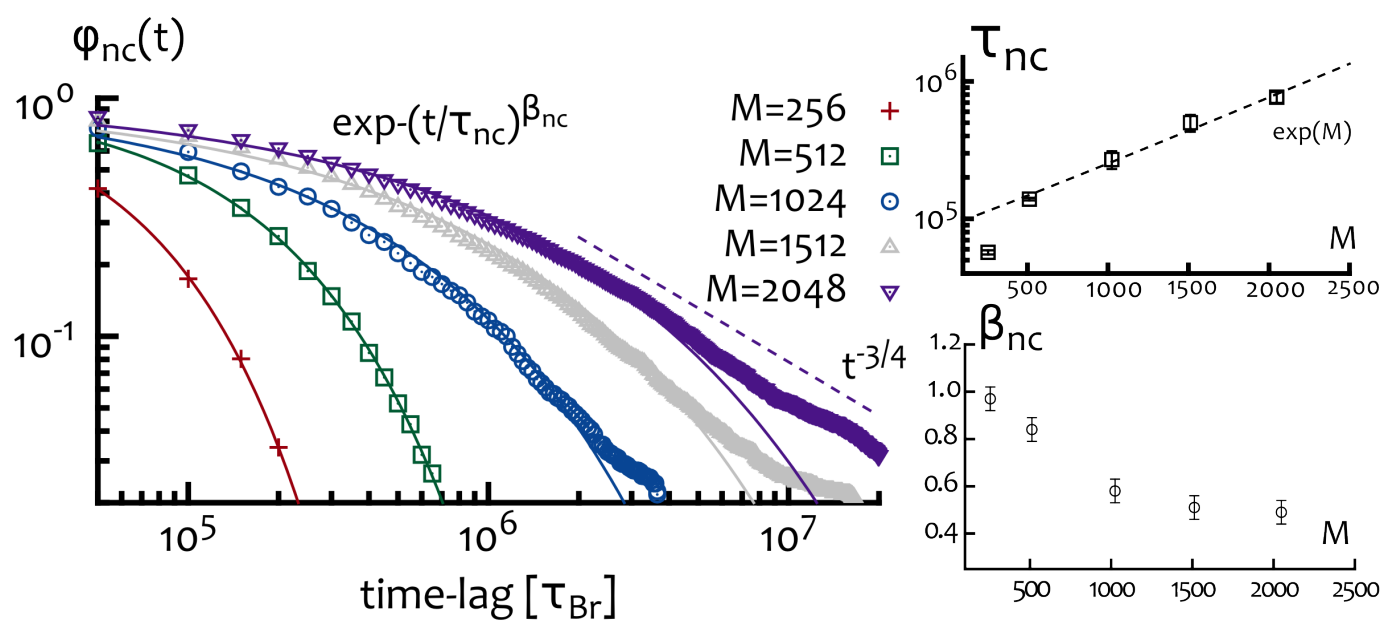

Figure 4.11: (Left) Fraction of persistent contiguous chains $\varphi_{n c}(t)$ is plotted against time for different chains length. It is worth noticing that while the decay for short chains is very well approximated by a simple exponential, for longer chains one observes (i) that the behaviour is well fitted by stretched exponentials (solid lines) and also (ii) the presence long-time fat tails. (Right Top) Values of $\tau_{n c}$ and of (Right Bottom) $\beta_{n c}$ used to fit the data points $\varphi_{n c}(t)$ with stretched exponentials.

with $\beta_{\text {nc }}$ decaying from $\beta_{\text {nc }}(M=256)=1$ to $\beta_{\text {nc }}(M=2048) \simeq 0.5$ (see Fig. 4.11). The values of $\tau_{\text {nc }}$ instead show a striking exponential increase which suggests the rapid emergence of slow dynamics in the exchange dynamics of the rings. Stretched exponentials with stretching exponents smaller than one and exponentially increasing relaxation times are both strong signatures of the onset of glassy dynamics and dynamical heterogeneities in glass-forming systems [Berthier and Biroli, 2011] and it encourages further investigations into the inter-coil correlations in systems of rings.

It is also worth noticing that the decay time of $\varphi_{\text {nc }}(t)$ is conceptually intimately related with the tube renewal time for a system of linear polymers. In fact, one can imagine that very persistent contiguous chains must possess some level of correlation that does not allow them to drift away from each-other, similarly to the chains forming the reptating tube of any one linear polymer. One can therefore argue that the motion of contiguous chains is strongly correlated and one possible cause might be the presence of threadings. Unfortunately, it is not possible to directly check if this is the case in this system, as opposed to the case of rings embedded in a gel.

Finally, it is worth stressing that while linear polymers cannot fully renew their conformation until they decorrelate from the chains forming their tube, there is no explicit constraint for ring polymers to relax the spatial stress stored along their contour by, for instance, renewing their protruding segments (see $G(t)$ in Fig. 4.5 and $\tau_{\text {diam }}$ compared with $\tau_{\text {relax }}$ in Fig. 4.6), while retaining proximity with contiguous chains. In other words, the type of "entanglement" at which ring and linear polymers are subjected to in dense solutions is rather different and one can speculate it to have a strong "topological" character. 


\subsubsection{Inducing a Topological Glass by Randomly Pinning Rings}

The major problem that one faces when studying threadings in solutions of rings is that this topological interaction is not well defined and virtually impossible to identify and characterise in this condition. As a consequence, the effect of threadings on the dynamics of the rings is even more elusive and for this reason very poorly understood. This is why in this Section I will attack this problem from an unconventional perspective. I will in fact make use of techniques adopted by studies on glass-forming systems and in particular I will take inspiration from a number of recent papers that advanced the idea, and investigated the behaviour, of glassforming systems under an external pinning field Bouchaud and Biroli, 2004, Biroli et al., 2008, Cammarota and Biroli, 2012, Karmakar and Parisi, 2013, Cammarota, 2013. Gokhale et al., 2014, Nagamanasa et al., 2015, Ozawa et al., 2015.

In these systems a fraction $c_{p}$ of constituents are artificially frozen in time and the response of the system to this perturbation is studied. One of the most striking findings is that the glass transition temperature of prototypical glass formers such as spin-glasses display a dependence on the value of the pinned fraction $c_{p}$ Cammarota and Biroli, 2012]. In the case of spin glasses, this effect has been conjectured to be intimately related with the fact that pinning spins reduces the configurational entropy of the system. This means that the systems are more prone to reach the ideal glass-transition state (at which the systems displays zero configurational entropy) at temperatures higher than the natural $T_{g}$ Cammarota and Biroli, 2012, Karmakar and Parisi, 2013, thereby facilitating their experimental and numerical investigation. Several protocols have been used to probe the response of systems to this artificial perturbation, and in particular, different geometries have been adopted where the frozen fraction of constituents, spins or particles, are distributed either on a plane Nagamanasa et al., 2015, randomly Cammarota and Biroli, 2012, Gokhale et al., 2014] or to form cavities Mézard and Parisi, 2001, Cammarota, 2009.

The Physics underlying the glass-transition is still very much debated. In particular, whether it can be interpreted as an ideal thermodynamic transition, or solely a kinetic one, is a long debated topic. For sure, systems in which the glass-transition is driven by pinning a fraction of the constituents display a high level of correlations and possibly, complicatedly structured "cooperatively rearranging regions" (CRRs) NNagamanasa et al., 2015]. The existence and growth of these regions when $T \rightarrow T_{g}$ had been suggested 50 years ago by Adam and Gibbs Adam and Gibbs, 1965 in a seminal paper. For instance, in a glass-forming system made of colloidal particles, CRRs are made of particles surrounding any one particle and allow the macroscopic motion of this particle by cooperatively re-arranging their position. As the temperature (or the density) approaches the glass-transition one, these regions grow in size thereby freezing the position of the particle at the centre of the cluster, limiting its motion to local vibrations within a cage. Analogously, if one believes 

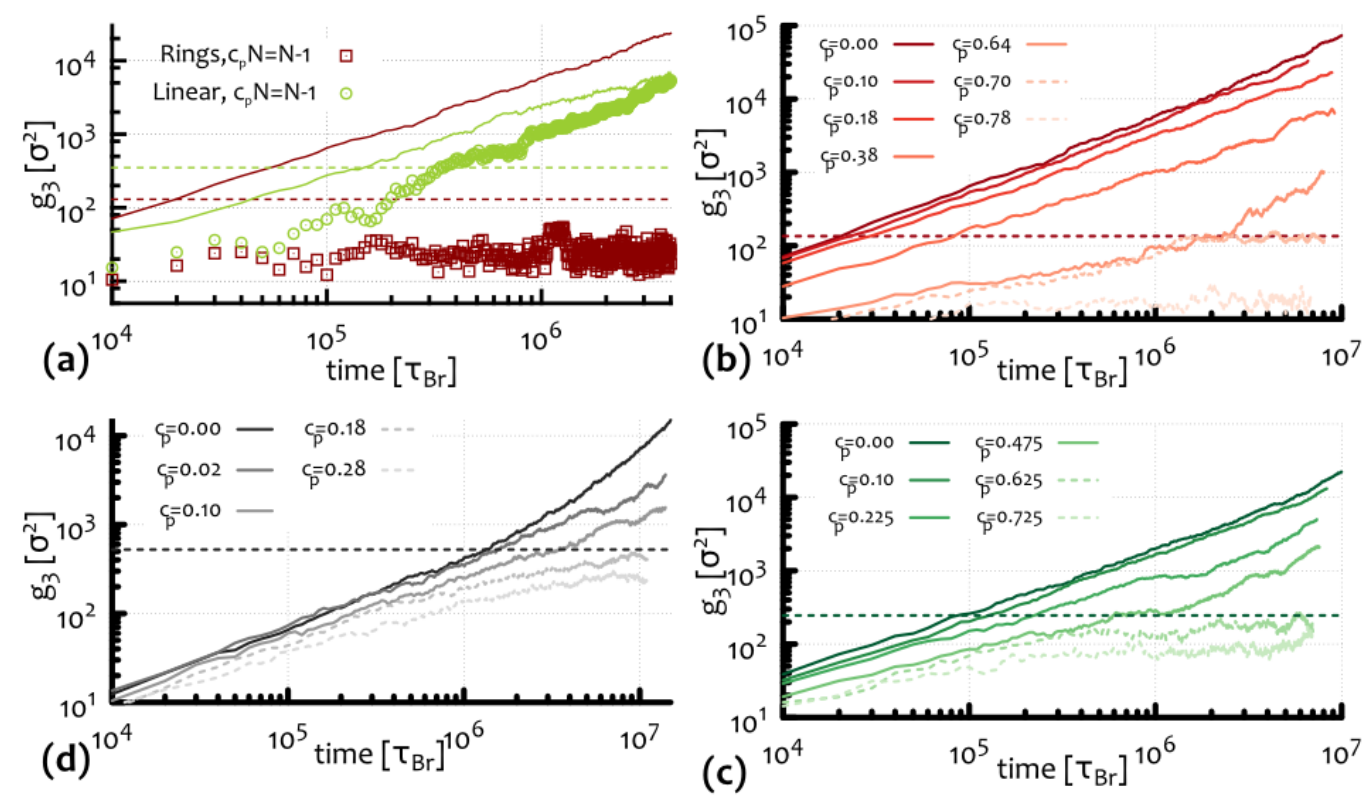

Figure 4.12: (a) Mean square displacement of the centre of mass $g_{3}(t)$ of polymer diffusing through $N-1$ frozen polymers. The data points are averaged over many choices of the probe. Lime data points represent linear polymers while red data points represent system of ring polymers both systems are chosen with $M=256$ and $N=50$. The corresponding mean squared displacement for the two systems in the case where no polymer is frozen $(c=0)$ is also shown as solid lines. (b)-(d) Mean square displacement of the centre of mass $g_{3}(t)$ for different choices of $c_{p}$ and for different systems: (b) $M=256$, (c) $M=512$ and (d) $M=1512$. The kinetically arrested state $c_{p}=c_{p}^{\dagger}$ is reached when the un-frozen polymers cannot diffuse further than $R_{g}$ and the corresponding $g_{3}$ are indicated as dashed lines. Dark horizontal dashed lines represent $\left\langle R_{g}^{2}\right\rangle$ for the different cases.

that rings in the melt, or in dense solutions, assume inter-threaded configurations as pictured in Fig. 4.8(b) then CRRs can be conceptually related to the longest sequence of rings that needs to come undone for the black ring to fully renew its neighbours. When a fraction $c_{p}$ of constituents in a glass-forming system is frozen, this contributes, in a complex way, to increasing the size of CRRs. In fact, freezing all the neighbours around a probe particle would mean creating an infinitely large CRR around that particle.

In light of this picture it is natural to ask the following question: what happens if one starts to randomly pin a fraction $c_{p}$ of rings in a solution of rings? or even, what happens if one artificially freezes all the rings apart from one?

If one reasons from a standard reptation theory point of view Doi and Edwards, 1988, then the prediction is that the probe polymer will simply snake or diffuse trough the frozen obstacles. This is in fact what one observes in the case of systems of linear polymers (see lime data points in Fig. 4.12(a)), where in the limit of large time-scales the diffusive behaviour is nearly ${ }^{\text {** }}$ indistinguishable when compared with

\footnotetext{
${ }^{* *}$ Since the mobile chain leaves a hole that produces an uniformity in the system density and could, in principle, interfere with a perfectly free diffusion.
} 
the pure melt case $\left(c_{p}=0\right)$. On the contrary, by repeating the same in silico experiment on the corresponding system of equilibrated rings with same contour length, one observes a striking behaviour: the centre of mass of the probe (free) ring is forever trapped in a region smaller than $R_{g}$ (see red data points in Fig. 4.12(a)).

This remarkable difference is due to the topology of the constituents, as no other parameter has been changed. One can imagine that if the probe ring assumed conformations such as the moose in Ch. 1 (Fig. 1.2(a)), i.e. not threaded by other rings, then it would simply diffuse through the frozen background by protruding its contour through the spaces, very much like predicted by the theories of ring polymers motion advanced in the 80's Rubinstein, 1986, Rubinstein, 1987. Cates and Deutsch, 1986 and more recently Milner and Newhall, 2010, Smrek and Grosberg, 2015. Otherwise, if the probe ring was threaded, such as the modified moose in Fig. 4.8(c)) or the black ring in Fig. 4.8(b) by its red, green and blue neighbours, then it is reasonable to expect its diffusion to be strongly constrained, or even completely hindered, by the fact that its neighbours become frozen.

A short threading segment is expected to contribute very little, as it can be easily circumvented, but if the threading segment was longer and itself threaded, that would created a much more persistent obstacle for the free ring and perhaps impossible to get rid of (especially if the sequence of rings that needs to be escaped forms a network larger than the ring size or, even, as large as the system size (see Sec. 4.1 and Fig. 4.7) ).

Similarly to what has been observed in glass-forming liquids when a region of the system was pinned by, for instance, optical traps Gokhale et al., 2014, Nagamanasa et al., 2015, here one can argue that it might be possible to exploit the topological interactions occurring within a dense solution of rings to generate a kinetically trapped subset of the system, for instance by adopting the following protocol: Starting from the unperturbed case, i.e. $c_{p}=0$, one can increase the value of $c_{p}$ until the mean square displacement of the centre of mass $\left(g_{3}(t)\right)$ of the remaining fraction $f_{p} \equiv 1-c_{p}$ of rings ceases to display free diffusion, i.e. $\lim _{t \rightarrow \infty} g_{3}(t) \sim$ const. This value of $c_{p}$, to which I will refer to as $c^{*}$, is the critical fraction of pinned rings for which all the other non-explicitly pinned rings are trapped by topological interactions, i.e. threadings. An example of this protocol is reported in Fig. 4.12(b)-(d) where I show the mean squared displacement of the rings centre of mass for different systems and for a range of $c_{p}$.

More formally, one can define $c_{p}^{\dagger}$ by introducing the following order parameter:

$$
R_{c}^{-1} \equiv \lim _{t \rightarrow \infty}\left\langle g_{3}(t)\right\rangle^{-1 / 2}
$$

which has dimensions of an inverse length and jumps from 0 to finite values only when the un-frozen rings are "caged" by the topological interactions. From this, it is therefore natural to identify $c_{p}^{\dagger}$ as the value of $c_{p}$ at which $R_{c}^{-1}$ displays the 

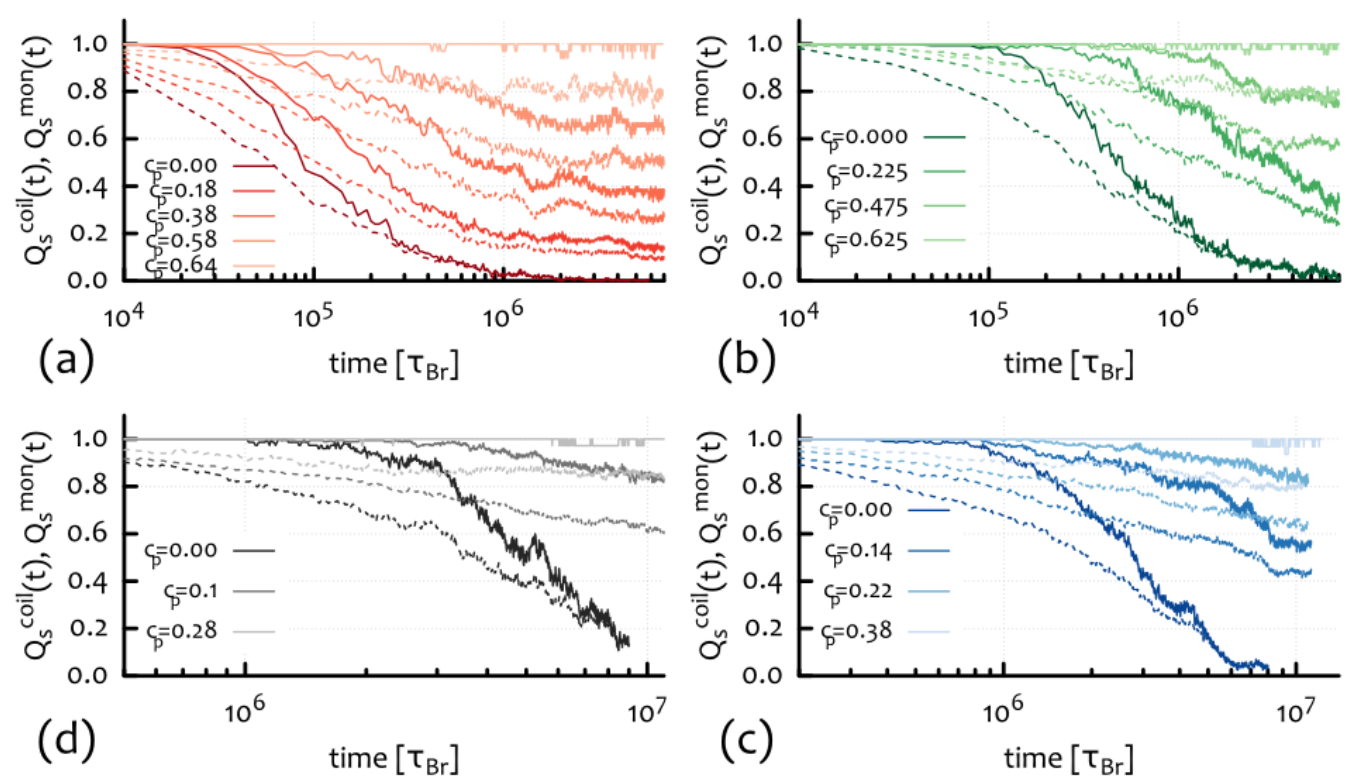

Figure 4.13: Overlap parameters $Q_{s}^{\text {coil }}\left(t ; c_{p}\right)$ (solid lines) and $Q_{s}^{\text {mon }}\left(t ; c_{p}\right)$ (dashed lines). It is worth noting that while the large time value of both $Q_{s}^{\text {coil }}\left(t ; c_{p}\right)$ and $Q_{s}^{\text {mon }}\left(t ; c_{p}\right)$ vanish at $c_{p}=0$, they display an arrested decay for larger $c_{p}$ until at $c_{p}=c_{p}^{\dagger}$ the overlap parameter $Q_{s}^{\text {coil }}\left(t ; c_{p}\right)$ equals unity at any time.

discontinuity. Another way of defining $c_{p}^{\dagger}$ that is perhaps more familiar to the common practice in the literature about the glass transition is by measuring the overlap parameter Karmakar and Parisi, 2013 for the coils

$$
Q_{s}^{\text {coil }}\left(t ; c_{p}\right)=\left\langle\frac{1}{f_{p} N} \sum_{I}^{\prime} \Theta\left(w-\left|\boldsymbol{r}_{C M, I}\left(t+t_{0}\right)-\boldsymbol{r}_{C M, I}\left(t_{0}\right)\right|\right)\right\rangle_{t_{0}}
$$

and for the monomers

$$
Q_{s}^{\operatorname{mon}}\left(t ; c_{p}\right)=\left\langle\frac{1}{f_{p} N} \sum_{I}^{\prime} \sum_{i \in I}^{M} \Theta\left(w-\left|\boldsymbol{r}_{i}\left(t+t_{0}\right)-\boldsymbol{r}_{i}\left(t_{0}\right)\right|\right)\right\rangle_{t_{0}},
$$

where $\sum^{\prime}$ indicates the sum restricted to the un-frozen $f N \equiv\left(1-c_{p}\right) N$ rings, $\Theta(x)$ is the Heaviside function and the window parameter $w$ is set to $2 R_{g}$ Karmakar and Parisi, 2013 since I am interested in averaging out the jiggling of the coils within cages of linear size $w=2 R_{g}$.

According to the interpretation of the arrested dynamics that I have given above in terms of cages formed by threadings, one expects that, as $c_{p}$ approaches $c_{p}^{\dagger}$,

$$
Q_{s}^{\mathrm{coil}}\left(t ; c_{p}^{\dagger}\right)=1 \forall t
$$

since the center of mass of the un-frozen coils start rattling inside cages of size $2 R_{g}$. At the same time, one also expects that the monomers are instead partially free to 
decorrelate from their initial state, giving

$$
0<\lim _{t \rightarrow \infty} Q_{s}^{\operatorname{mon}}\left(t ; c_{p}^{\dagger}\right)<1
$$

These predictions are nicely recovered from the explicit computation of the overlap parameters whose behaviour is reported in Fig. 4.13 .

At this stage it is important to stress another message that can be inferred from Figs. 4.12 and 4.13 . The value of $c_{p}^{\dagger}$ is dependent on the length of the polymers $M$. In fact, this seems to decrease when $M$ is increased, thereby suggesting that fewer and fewer rings need to be artificially frozen in order for the system to display the kinetically arrested state. Before investigating further the $M$ dependence of $c^{*}$, I will first discuss the coherent scattering function $S_{c}(q, t)$, that is often referred to in the glass-transition literature. This is here computed over the $f_{p} N=\left(1-c_{p}\right) N$ free rings and can quantify the relaxation, or decorrelation, associated with length scales $l=2 \pi / q$.

It is common practice to tune the scattering vector at values corresponding to $r_{\max }$, where $r_{\max }$ is the position of the peaks of the pair correlation function $g(r)$. In this case, it is more appropriate to tune it to values corresponding to the coils diameter $2 R_{g}$, since this is the length scale above which one is interested to probe the rings' dynamics. For this reason I here choose two values of $q$ : one corresponding to length-scales slightly greater than $2 R_{g}$, i.e. $q_{1}=2 / R_{g}$ and the second corresponding to length scales slightly smaller than $2 R_{g}$, i.e. $q_{2}=4 / R_{g}$. In Fig. 4.14 I report $S_{c}(q, t)$ for different values of $c$ and compare these choices of $q$ for systems made rings $M=512$ and $M=1512$ beads long.

As mentioned in Ch. 2, the scattering function is dominated by pair of beads that have not travelled much further than $2 \pi q^{-1}$ after $t$ time-steps, and for this reason it is more sensitive than $g_{3}(t)$ to the slow elements of the system. In Fig. 4.14 one can observe that when $c_{p}=0$, that is the case of an unperturbed system, the scattering function displays a decay at both values of $q$. When $c_{p}$ is increased, the scenario changes dramatically: $S_{c}\left(q_{1}, t\right)$ shows an arrested decay which is strongly reminiscent of those observed in glass-forming systems approaching $T_{g}$ Kob et al., 1997. On the other hand, shorter length-scales, i.e. longer wavelengths, show a much faster de-correlation as showed by $S_{c}\left(q_{2}, t\right)$. In particular, in this latter case, all the curves seem to differ very little from one another indicating that there is no (or little) effect of the increasing $c_{p}$ in the relaxation of those length-scales. These findings agree remarkably well with the "pierced lattice animal" representation. As I argued in Ch. 2, threadings that are not dense enough are in fact most likely to slow down the translational degrees of freedom, i.e. long length scales, while leaving shorter length scales free to relax the stress and re-arrange the rings' configuration.

While in the case of vitrifying systems the appearance of a plateau in the intermediate scattering function can have multiple and sometimes not well under- 

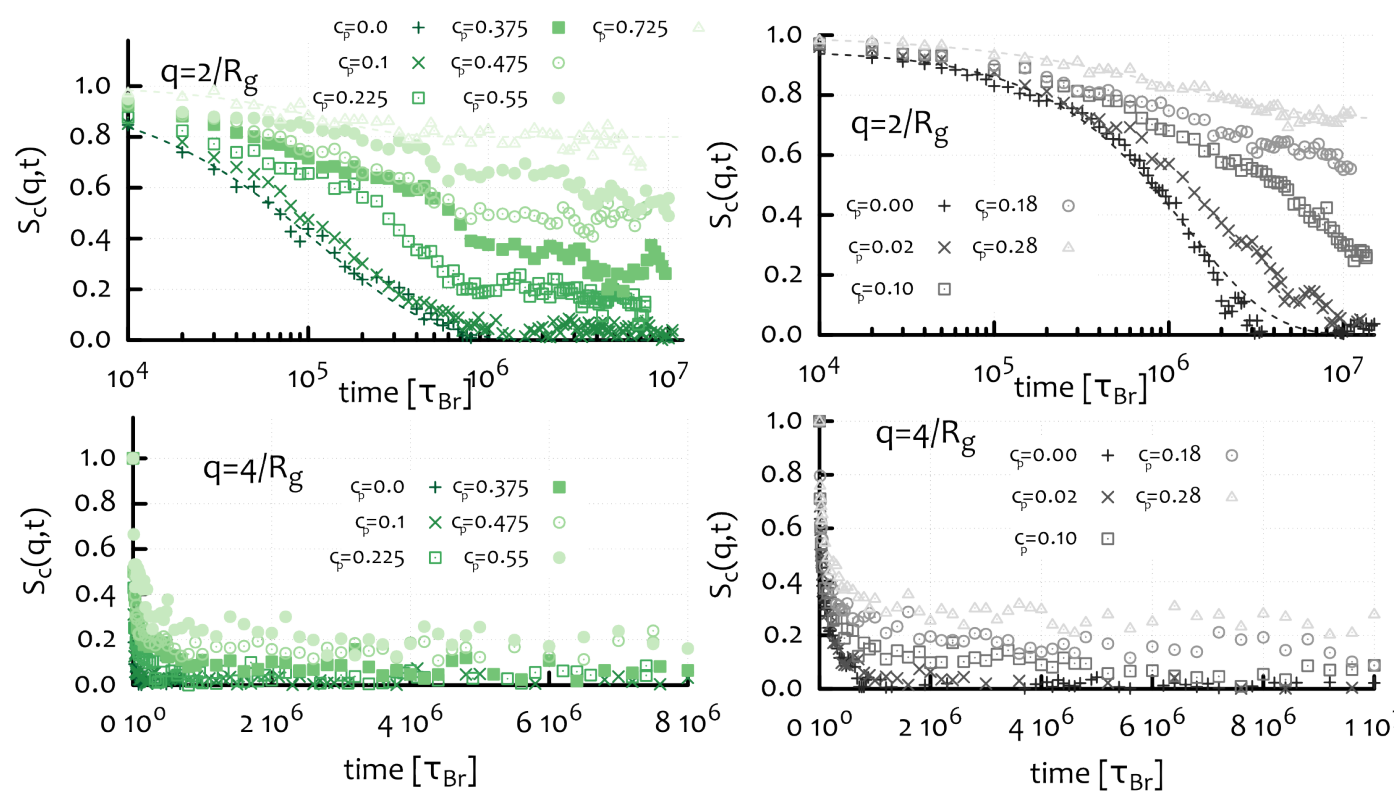

Figure 4.14: Coherent scattering function $S_{c}(q, t)$ (normalised by $S_{c}(q, 0)$ ) for different values of $c$ and for $q=q_{1}=2 / R_{g}$ (Top Row) and $q=q_{2}=4 / R_{g}$ (Bottom Row) for a system with $M=512$ (Left) and $M=1512$ (Right).

stood Berthier and Biroli, 2011] origins, in this case it can be explained in terms of threadings: above $c_{p}^{\dagger}$, length-scales $2 \pi q^{-1} \gtrsim \pi R_{g}$ display no relaxation at all, since the translation of the un-frozen rings at distances greater than $2 R_{g}$ is completely suppressed. On the other hand, length-scales shorter than $2 R_{g}$ can still relax and de-correlate, since rings are free to create new protrusions anywhere along their contour. This means that the two time-scales associate with internal organisation of the ring conformation (equivalent to $\tau_{\text {diam }}$ of the previous Section) and the diffusion of the centre of mass (equivalent to $\tau_{\text {relax }}$ of the previous Section), are decoupled.

In other words, the situation presented here is a clear example of how rings can relax their internal stress without displaying free diffusion, which is temptingly similar to the phenomenon that has been observed both numerically and experimentally for ring polymers in the melt Kapnistos et al., 2008, Halverson et al., 2011a. Pasquino et al., 2013 but for which a clear explanation was still lacking.

This scenario is also strongly reminiscent of $\alpha$ and $\beta$ relaxation modes in Mode Coupling Theory Aichele and Baschnagel, 2001, Berthier and Biroli, 2011 of glass transition. Caged polymers spend the majority of their time re-arranging their internal structure without performing macroscopic jumps. In this respect, ring polymers behave like particles in colloidal glass-forming systems as $T$ approaches $T_{g}$, for which the macroscopic motion ( $\alpha$ relaxation) decorrelates from the local jiggling ( $\beta$ relaxation). In particular, it is common in the glass-transition literature, to define a non-ergodic parameter $f_{c}(q)$ equal to the value of $S_{c}(q, t)$ at the plateau Aichele 
and Baschnagel, 2001. In the case studied here, one can simply take

$$
f_{c} \equiv \lim _{t \rightarrow \infty} S_{c}(q, t)
$$

computed for $q=2 / R_{g}$, as the decay of this scattering function is arrested at that value (Fig. 4.14 闯

Before continuing with the analysis of this system, I would like to stress, once again, that these features are not observed in system of linear polymers, in which an arbitrary fraction $c_{p}$ of polymers can be artificially frozen while virtually un-affecting the long-time behaviour of the other polymers. The features observed in the systems presented here are solely due to the rings topology, and are the first unambiguous signature of the presence of threadings between rings in solution.

\section{The Phase Diagram of the System Suggests the Emergence of a "Topo- logical Glass" in the Large M limit}

The behaviour of the non-ergodic parameter $f_{c}$ is reported in Fig. 4.15. It is clear that the transition from $f_{c}=0$ for $c_{p}=0$ to larger values of the non-ergodic parameter is sharper as the length of the rings increases. This implies that one requires a smaller and smaller perturbation parameter $c_{p}$ to "force" the system from a liquid-like to a glass-like one. The behaviour of $f_{c}$, when plotted against $f_{p}=1-c_{p}$ (see inset in Fig. 4.15) is well fitted by the functional form

$$
f_{c}=1-f_{p}^{\alpha}=1-\left(1-c_{p}\right)^{\alpha} \simeq \alpha(M) c_{p}
$$

in the small $c_{p}$ limit and where the exponent $\alpha$ seems to increase with the rings length $M$, indicating a quicker approach to large values of the arrested decay of $S_{c}(q, t)$.

Having defined rigorously defined $c_{p}^{\dagger}$ from $R_{c}$ in eq. (4.18) or, equivalently, using the overlap parameter $Q_{s}^{\text {coil }}$, from eq. (4.21), it is possible to obtain the functional form of $c_{p}^{\dagger}(M)$, that is reported in Fig. 4.15 .

In addition, one can use the large time value of the coils overlap parameter to define

$$
F_{s} \equiv \lim _{t \rightarrow \infty} Q_{s}^{\text {coil }}(t)
$$

which is a good estimation of the closeness of the system to $c_{p}^{\dagger}$ (recall that $F_{s}=$ 1 at $c_{p}=c_{p}^{\dagger}$ ). The behaviour of $c_{p}^{\dagger}(M)$ and $F_{s}$ is reported in the phase space

\footnotetext{
${ }^{\dagger \dagger}$ It is perhaps interesting to think about what would happen if one were to relax the uncrossability condition imposed on the chains and substitute it with a potential barrier of finite height $A$. In this scenario, one expects the scattering function to re-establish its decay although only after a time $t \sim\langle T h\rangle \exp A$ that corresponds to an activated process where at least some of the threadings must be by-passed. In this picture, the system would probably display a long-time $\alpha$ relaxation which decouples from the local rattling proportionally to the number of threadings in the system.
} 


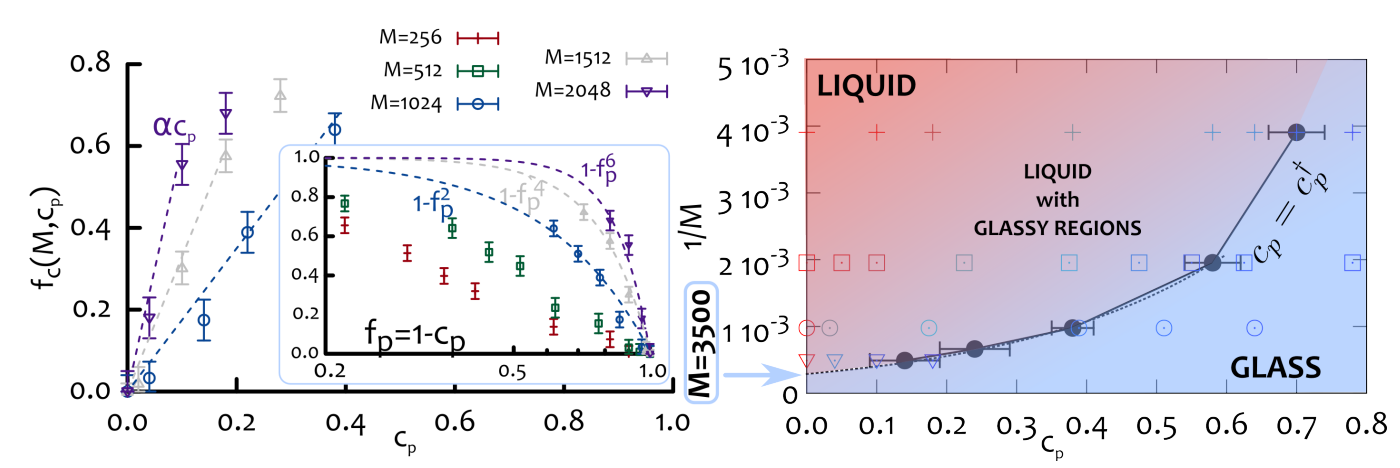

Figure 4.15: (Left) Functional behaviour of $f_{c}$ plotted against $c_{p}$ and $f_{p}=1-c_{p}$ in the inset. The behaviour is well fitted by $f_{c}=1-f_{p}^{\alpha}$ with $\alpha$ increasing with the length of the rings. (Right) Phase space diagram for a systems with rings $M$ beads long and where a fraction $c_{p}$ of rings have been artificially frozen in space and time. The coloured data points indicate the value of $F_{c}$ defined in eq. 4.25) which goes from $F_{s}=0$ (red) at $c_{p}=0$ to $F_{s}=1$ (blue) at $c_{p} \geq c_{p}^{\dagger}$. The fit through the last four data points indicates an exponential behaviour of the transition line whose $c_{p} \rightarrow 0$ limit gives $M_{g} \simeq 3500$ at which a spontaneous glass might emerge.

$(c, 1 / M)$. The straightforward interpretation that comes out from this plot is that the transition line $M^{\dagger}(c)$, or $c_{p}^{\dagger}(M)$, divides the parameter space into a liquid-like and glass-like regions. Furthermore, from this plot it is clear that the limit $c_{p} \rightarrow 0$ is an interesting one. In fact, at $M^{\dagger}\left(c_{p} \rightarrow 0\right) \equiv M_{g}$, the system is conjectured to display a dramatic response to a tiny perturbation, eventually leading to the emergence of a spontaneously vitrifying system.

The values of $c_{p}^{\dagger}$ for the four systems with largest chains follow, remarkably well, an exponential of the form

$$
M^{\dagger}\left(c_{p}\right)=M_{g} e^{-3.3 c_{p}^{\dagger}}
$$

with

$$
M_{g} \equiv \lim _{c \rightarrow 0} M^{\dagger}\left(c_{p}\right) \simeq 3500 .
$$

Although the precise value of $M_{g}$ might suffer from the finite size of the simulated systems, the general behaviour of the transition line encourage the conjecture that in the limit of large polymerisation index $M$, a spontaneous glass might occur.

Even though performing simulations with such big $M$ or purifying solutions of very long rings might be too difficult with the current technology, the results that I reported here strongly encourage the experimental realisation of the perturbation protocol employed in this Section. The response to this perturbation should in fact become more and more dramatic as the length of the rings increases, eventually requiring only a small fraction of rings to be frozen to cause the whole system to be kinetically trapped.

One can furthermore argue that this perturbation protocol might be experimentally realised by using optical traps as in recent experiments on colloidal glass forming systems Gokhale et al., 2014. Another option for its experimental realis- 
ability could be considering a mixture of polymers having different freezing temperatures. In this scenario, the parameter $c$ could be directly related to the mixture concentration and temperature of the system. Another option could be considering a bi-disperse system or blends, where longer and heavier (and slower) ring polymers are in solutions with lighter ones. By changing the ratio of the concentrations one could in principle control an equivalent $c$ parameter also in this case.

It is finally worth stressing once again that all this is performed at temperatures where the thermal energy equals the LJ interaction energy (the Langevin thermostat is fixed to $T=\epsilon / k_{B}$ ) and at fixed monomer density $\rho=0.1 \sigma^{-3}$. This implies that the diagram reported in Fig. 4.15 is one of the first instances of vitrification occurring in a system where neither temperature, or density, are the main control parameters used to drive the glass-transition. This is in fact solely driven by topological interactions, and for this reason it cannot be observed in systems of topologically trivial polymers such as linear ones.

One should finally emphasise that the transition observed in these systems is not a thermodynamic one, i.e. there is no divergence of thermodynamic quantities. Although it is clear that at $c_{p}^{\dagger}$ the system displays a macroscopically different behaviour, this is only due to the topological interactions present in the system, which cause kinetic traps. In this respect, one can talk of "topological correlations" which, in the case studied here, are the main players controlling the purely kinetic glass transition佣

\subsection{Conclusions}

In this Chapter I have tackled the problem of investigating topological interactions in solutions of un-linked and un-knotted ring polymers, i.e. threadings. In the first section of this Chapter I showed that these are readily studied when the solution of rings is forced to move inside a static gel structure and I described a novel method to quantify ring-ring penetrations by exploiting its architecture. This method allows one to identify and quantify both, inter-ring threadings and their effect on the rings dynamics. On the other hand, it is worth highlighting that the gel structure can have a non-negligible effect the rings. The presence of the grid, and the consequent topological constraint that acts on the rings (as they have to be unlinked from it at any time), might play an important role on the equilibrium configurations of the rings, which might display more elongated and squeezed shapes, with respect to those assumed in absence of the gel. In addition, the relaxation of the rings might be slowed down by the facts that the rings have additional constraints to respect, on top of those presented by the neighbouring chains. As I will show in the next

\footnotetext{
${ }^{\ddagger \ddagger}$ and is broadly analogous, although in a much less coarse-grained sense, to what happens in glass-forming systems belonging to the class of "Kinetically Constrained Models" Palmer et al., 1984
} 
chapters, the presence of a gel and its detailed microscopic structure, plays a crucial role in the out-of-equilibrium dynamics of ring polymers (see Ch. 6). When an external force is driving the polymers, they can in fact get impaled and permanently entangled. While the effects of the gel on the equilibrium properties of the rings might be less dramatic, quantifying their relevance would nonetheless be important. A more detailed investigation of these effects would be a natural continuation of the work presented in this Chapter. This could readily be done by investigating systems in which the gels possess lattice spacings varying from $l \ll l_{K}$ to $l \gg l_{K}$. In the former limit, the rings are expected to be in the regime of extreme elongation, while in the latter the system properties could readily be compared to those observed in a pure melt of rings.

The most important and remarkable result of the first Section is that threadings are a critical quantity, in that they extensively grow with the length of the rings. It is also worth stressing that I have showed the existence of connected clusters of inter-threaded rings, whose size grows with the number of threadings in the system. A cluster of inter-penetrating rings of size $\mathcal{O}(N)$ has been showed to emerge for the longest chains that I investigated. Correspondingly, the same system displays a dramatic slowing down of its diffusion (see Fig. 4.6.

Although the results presented in the first Section of this Chapter are formally bound to hold in the case of a dense solution of rings immersed in a static gel structure, it is worth noting that many of the reported findings are in agreement with those for a pure melt of rings. In particular three quantities are worth comparing: (i) the scaling of the radius of gyration $R_{g}^{2} \sim M^{2 \nu}$ with $\nu=1 / 2$ for short rings and $\nu=1 / 3$ for long ones, is in perfect agreement with recent MD simulations of a melt of rings Halverson et al., 2011a, Rosa and Everaers, 2014 and theoretical predictions for the melt case (see Ch. 2); (ii) the segmental displacement, reported here to reach free diffusion only when rings have travelled many times their own size $\left\langle\delta r_{s}^{2}\right\rangle /\left\langle R_{g}^{2}\right\rangle>1$, and to display a slowing down $\left\langle\delta r_{s}^{2}\right\rangle \sim t^{1 / 4}$ for $M=1512$ is in agreement with Halverson et al., 2011b; ( $i i i)$ the stress relaxation, found to decay as a power law $G(t) \sim t^{0.4}$ and on time-scales much shorter than the overall relaxation of the chain (free diffusion) is in agreement with experimental and numerical evidence for the melt Kapnistos et al., 2008, Halverson et al., 2011b. All this strongly encourages the speculation that, after all, the system studied here is not too far from a melt of rings. In particular, the topological constraints to which the chains are subjected to in the case when a gel is present can be thought of as constraints coming from other chains in the melt. This conjecture is in part similar to what was assumed in the literature Cates and Deutsch, 1986, Rubinstein, 1986. Obukhov and Rubinstein, 1994 when the authors investigated melt of rings by placing a background of obstacles surrounding the rings (but not threading them!).

In the second Section of this Chapter I focused on probing threadings in dense 
solutions of rings. Because no current algorithm is capable of unambiguously identifying threadings in this situation, I have tried to investigate this system from an unconventional point of view, hoping, and to my opinion, managing, to detect signatures directly associated with threadings.

Firstly, I showed that rings in dense solutions display a high level of inter-coil correlations (via measuring the contiguity persistence $\varphi_{\mathrm{nc}}(t)$ reported in Fig. 4.11). These correlations have then been further explored by probing the kinetics of the systems under an external perturbation. This has been implemented by freezing in space and time a fraction $c_{p}$ of coils in the system. The response of different lengthscales to this perturbation has been measured by computing the coherent scattering function $S_{c}(q, t)$ on the fraction $f_{p}=1-c_{p}$ of non-frozen rings. This showed that while length scales $l \simeq 2 R_{g}$ are strongly affected by the frozen fraction of rings, shorter length scales are less affected, implying that while the translational degrees of freedom of the rings are suppressed by the frozen rings, internal relaxation modes are largely unhindered. This has been interpreted as a strong signature of the fact that the inter-coil correlations take the form of threadings.

Finally, I showed that the critical fraction of frozen rings $c_{p}^{\dagger}$ needed to drive a kinetically trapped state for all the remaining $f N=\left(1-c_{p}\right) N$ rings displays a strong dependence on the length of the rings $M$. By plotting the transition line $c_{p}=c_{p}^{\dagger}$ in the phase space $\left(c_{p}, 1 / M\right)$ I then showed that the results from the simulations imply that the limit $c_{p} \rightarrow 0$ gives a critical value for $M\left(c^{\dagger}\right) \equiv M_{g}$ at which a spontaneous glass might emerge.

I hope that these results motivate further investigations into this interesting problem. In particular, I conjecture that novel experimental set-ups that have very recently been used to probe the glass-transition in colloidal and molecular liquid systems Gokhale et al., 2014, Nagamanasa et al., 2015 might be used to put into practice the perturbation protocol proposed here and finally prove the existence of this long sought "topological glass". 
Sometimes careful physics-based

thinking can illuminate

complicated issues in biology

M. E. Cates

\section{A Bio-Physical Model for the Kinetoplast DNA}

\section{Contents}

5.1 KDNA as an Ensemble of Diffusing Phantom Loops . . 73

5.2 Probing the Network Topology . . . . . . . . . 75

5.3 An in silico Digestion . . . . . . . . . . . . . . . 79

5.4 A Linked Network of Rings at the Percolating Point . . 83

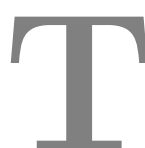

HE Kinetoplast DNA (or KDNA) Simpson, 1967 is one of the most complex and singular forms of DNA in nature. It is uniquely found in the mitochondrion of a group of unicellular eukaryotic organisms of the class Kinetoplastida. Some of these organisms have been studied since the late '60s because they are responsible for several serious diseases such as sleeping sickness and leishmaniasis Young and Morales, 1987, and are among the earliest diverging eukaryotic organisms containing a mitochondrion Avliyakulov et al., 2004.

The Kinetoplast DNA is made of thousands $(\sim 5000)$ of short $(1-2.5 \mathrm{kbp})$ DNA loops which are interlinked to form a large network. The short loops, or "mini-circles", are also linked with fewer ( 30) larger loops, or "maxi-circles", consisting of around $30-50 \mathrm{kbp}$ Shapiro and Englund, 1995]. The maxi-circles contain standard mitochondrial genetic material, which is made readily translatable only thanks to the guide RNAs (gRNAs) encoded in the mini-circles. These have in fact been found to be mostly made by RNA-editing genes and to be genetically heterogeneous, i.e. few genetic sequences are repeated in every mini-circle Lukes et al., 2005, Lai et al., 2008, Jensen and Englund, 2012. . C. fasciculata mini-circles assemble in a network whose shape resembles that of a disc. Its dimensions have been found to be around $1 \mu \mathrm{m}$ in diameter and $0.4 \mu \mathrm{m}$ in thickness Pérez-Morga and Englund, 1993b, Lukěs et al., 2002, Jensen and Englund, 2012. Networks which are removed from the mitochondria, e.g. via cell lysis, expand into an elliptical shape 

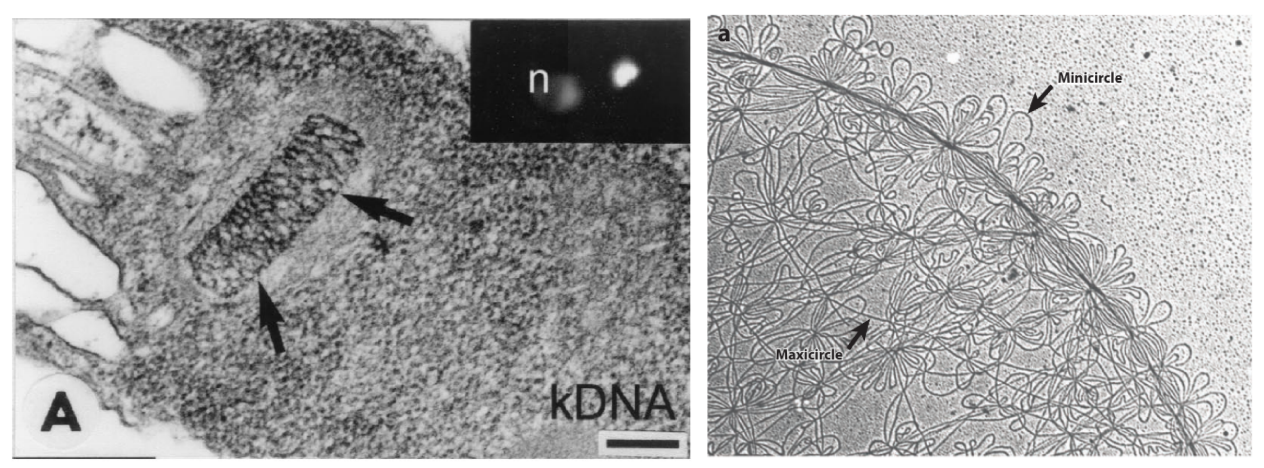

Figure 5.1: (left) Electron micrograph of a section of $C$. fasciculata in vivo. The Kinetoplast DNA strucutre is indicated by arrows (Ref. Lukeš et al., 2002]). (right) Isolated Kinetoplast DNA; the arrow in the top left corner indicates a mini-circle while the other arrow points to a maxi-circle (Ref. Jensen and Englund, 2012]). The scale-bar is $200 \mathrm{~nm}$.

whose minor and major axis are respectively around $10 \mu \mathrm{m}$ and $15 \mu \mathrm{m}$, i.e. roughly a hundred times bigger than their dimension in vivo Jensen and Englund, 2012. This suggests that the networks experience a confinement within the mitochondria (see Fig. 5.1).

A "tripartite attachment complex" (TAC) keeps the Kinetoplast statically in place near the basal body, from which it is physically separated by the mitochondrial envelope Ogbadoyi et al., 2003. Electron microscopy images of Kinetoplast networks in vivo Renger and Wolstenholme, 1971, Renger and Wolstenholme, 1972, Lukes et al., 2002, Ogbadoyi et al., 2003, Gluenz et al., 2007, Lai et al., 2008, de Souza et al., 2009 Docampo et al., 2010 show the mitochondrion as an elongated organelle connected to a bulge containing the Kinetoplast via a narrow neck (see Fig. 5.2. This strongly suggests that the network experiences a geometric confinement within a specific region of the mitochondrion. The mitochodrial envelope can therefore be thought of as acting a physical constraint on the outer structure of the network, while it is likely that histone-like proteins, such as p16, p17 and p18, or "KAP proteins" encoded in genes KAP2, KAP3 and KAP4, act as chemical constraint on the inner structure $X u$ and Ray, 1993, Xu et al., 1996, Hines and Ray, 1998, Silver et al., 1986, Lukeš et al., 2001, Avliyakulov et al., 2004.

The concentration of DNA in the Kinetoplast has been found to be around $50 \mathrm{mg} / \mathrm{ml}$ Shapiro and Englund, 1995], similar to that measured in bacteria (20 $\mathrm{mg} / \mathrm{ml}$ ) but far smaller than the one inside the head of a T4 bacteriophage (800 $\mathrm{mg} / \mathrm{ml}$ or more) Kellenberger et al., 1986. This suggests that the mini-circles are overlapping but there is considerable space between DNA strands Shapiro and Englund, 1995. Previous findings showed that the loops are linked only once with their neighbours Chen et al., 1995b. In addition, the valence of each ring, i.e. the number of linked neighbours, has been found to be around 3 in a pre-replicating network and approaching 6 at the end of duplication and before the network is split into the two daughter cells Chen et al., 1995b, Chen et al., 1995a, Liu et al., 2005. 


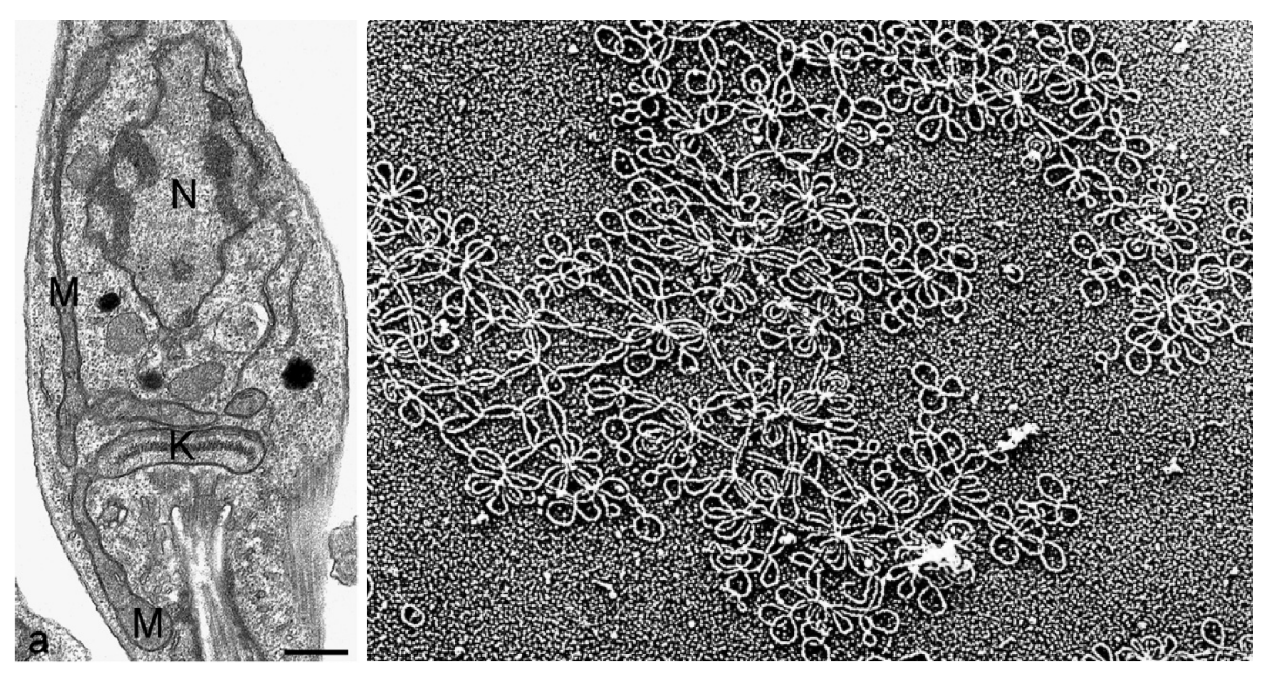

Figure 5.2: (Left) Electron micrograph of an ultrathin section of L. amazonensis. One can notice that the elongated strucutre of the mitochondrion (M) is connected to a bulge containing the Kinetoplast (K) via narrow necks (Ref. de Souza et al., 2009). (Right) Electron migrograph of a cluster of mini-circles isolated after cell lysis (Ref. [Simpson and Da Silva, 1971]). The scale-bar is $500 \mathrm{~nm}$.

This has been thought of as a consequence of the fact that during replication the Kinetoplast doubles the number of mini-circles, while retaining roughly the same volume. Only after that the network has been fully duplicated, the cell increases its volume and separates into two progeny cells. In light of this, the density of mini-circles is expected to play a major role in the network topology Diao et al., 2012. In addition, the mini-circles have been observed to be in a non-supercoiled state Rauch et al., 1993. This has been conjectured to be a consequence of evolutionary pressure: because relaxed loops are more likely to form linked structures than supercoiled ones Rybenkov et al., 1997, some of these organisms seems to have traded supercoiling in order to preserve the network linkedness Rauch et al., 1993.

During replication, catenation between the loops represents a non-trivial topological problem, which is solved as follows: First Topoisomerase II disentangles one loop at a time from the network Shlomai, 1994, Liu et al., 2005], the loop then diffuses through a region called the Kinetoflagellar Zone (KFZ), where the duplication process begins. The latter is then always completed at the "anti-podal sites" Drew and Englund, 2001, specific regions that flank the Kinetoplast where a higher concentration of ligase, polymerase and topoisomerase enzymes has been observed Melendy et al., 1988, Morris et al., 2001. Finally, the two progeny minicircles are re-attached to the periphery of the network Pérez-Morga and Englund, 1993b. The Kinetoplast in C. fasciculata has been found to spin relatively to the anti-podal sites Pérez-Morga and Englund, 1993b, Liu et al., 2005, and the newly duplicated mini-circle become re-attached with some lag-time with respect to the original mini-circle, most likely to allow specific enzymes to fill the gaps between 
DNA sequences Shlomai, 1994. This process has the side-effect to ensure an uniform distribution of the genetic material contained in the mini-circles throughout the network Liu et al., 2005. Both mini-circles are catenated to the network in a nicked state. The nicks along one of the two DNA strands have been conjectured to act as "bookkeepers" Englund, 1978, Englund, 1979, Kitchin et al., 1984. Thanks to this mechanism, it is in fact very likely that mini-circles can be safely duplicated only once during the replication of the network Shlomai and Linial, 1986.

During this process, the physical size of the network has been observed to remain constant, while the number of mini-circles contained and the valence of each minicircle to double Chen et al., 1995a. At the end of duplication, around the stage of cell division, the volume of the Kinetoplast doubles and the valence of the network is brought back to 3, thanks to topoisomerase enzymes, the nicks and gaps in the mini-circles are repaired Englund, 1978 and two copies of the network are produced by slicing the network through the middle, once again, most likely mediated by topo II Pérez-Morga and Englund, 1993b, Liu et al., 2005].

The organisation and duplication of the KDNA has the unique feature that it must undergo complex topological changes in a precise and consistent order. This could not be done without the action of topoisomerases, which is crucial for this machinery to work correctly, not only for decatenation of mini-circles from the network, but also for the re-attachment at the end of duplication Shlomai and Zadok, 1983, Chen et al., 1995b. RNA interference (RNAi) experiments showed that by suppressing the production of topoisomerase enzymes, the Kinetoplast is unable to form and most of the mini-circles remain in a free (unlinked) state Wang et al., 2000. As a consequence, the progeny cells are typically malfunctioning and likely to die. Type II topoisomerase is well-known for playing a crucial role in simplifying knots and catenanes which occur in DNA White et al., 1987); On the other hand, it has also been shown that the same enzyme is also capable of creating complex, linked structures Hsieh and Brutlag, 1980, Kreuzer and Cozzarelli, 1980, Brown and Cozzarelli, 1981, and its action to be tuned by a number of factors, such as DNA concentration or condensation mediated by the presence of polyamines, e.g. spermidine Krasnow and Cozzarelli, 1982.

All this strongly suggests that, on one hand, the KDNA is a complicated biological structure regulated by many subtle biological processes. On the other hand, seen from a purely physical perspective, the KDNA is represented by a state in which loops are linked to each other to form a connected component that spans the entire genetic repertoire. In particular, the presence of topoisomerase enzymes regulates the ability of loops to link to, or unlink from, one another thereby tuning the ability of the network to form. 
In what follows I will present a minimal model which aims to capture the essence of this complicated structure and in particular its topological organisation. Despite the level of complexity shown by this biological structure, I will approach it with a philosophy of aggressive simplification. The model I propose will mainly focus on what I consider the key physical ingredients driving the system organisation: $(i)$ the confinement on the outer structure of the network due to the mitochondrial envelope and (ii) the role of topoisomerase enzymes in re-modelling the inner linked structure. I will show that these two elements are of paramount importance in order to capture the network topology, and sufficient for obtaining many experimental results. In this Chapter I will consider, as a reference, KDNAs that are at the end of their duplication stage and are about to the separated into the two daughter cells. At this stage no duplication occurs, but only topological re-arrangements. The duplicating stage of the KDNA is a much more complex one, as other biological ingredients come into play (in Appendix C I provide an attempt to capture the topological regulation of the KDNA during this stage based on an analytical model). I will show that despite (or thanks to) their simplicity, both these models (reported in this Chapter and Appendix C) are able to provide us with some insight into this unique genomic structure and to shed some light onto its evolutionary survival.

Finally, it is worth mentioning that in this Chapter I investigate a system in which the regulation of linking between loops affects the survival of the organism. These take apart their KDNA and re-form it at every replication cycle with very few, or no, mistakes. I believe that it would be very instructive to learn more about how this is done in nature by these organisms. Many materials that find useful practical applications are in fact inspired by nature. The so-called "bio-mimetic materials" are more and more common and, in this case, the artificial making, for instance via synthetic biology methods, of a Kinetoplast would lead to the first ever in silico production of an "Olympic gel" de Gennes, 1979.

\subsection{KDNA as an Ensemble of Diffusing Phantom Loops}

The Kinetoplast DNA is here modelled as $N=50$ semi-flexible and torsionally relaxed ring polymers (see Ch. 3 for the Computational Details) made of $M=128$ beads of nominal size $\sigma$. As usual, I take $\sigma=2.5 \mathrm{~nm}$ as the diameter of the hydraded B-form of DNA near physiological conditions Rybenkov et al., 1993, and the length of the rings can be mapped to real units as $M \sigma=128 \sigma=320 \mathrm{~nm} \simeq 1 \mathrm{kbp}$, and the persistence length is chosen in order to match that of a double stranded DNA under physiological conditions, i.e. $l_{p}=20 \sigma=50 \mathrm{~nm}$.

The outer confinement due to the mitochondrial envelope is implemented by confining the system into a box of linear size $L$ in units of $\sigma$ and implemented via a purely repulsive Lennard-Jones interaction between the beads forming the polymers and the walls situated at the boundaries of the simulation box. In what follows, I 

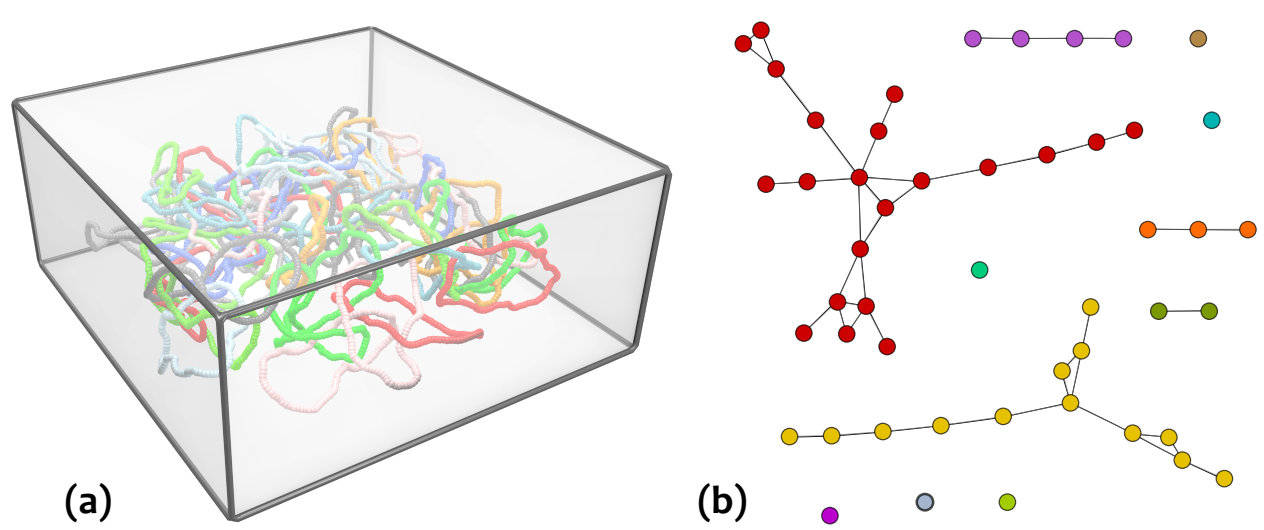

Figure 5.3: (a) Graphical representation of the system. The colors of the rings are chosen randomly for clarity of visualisation. (b) Network representation of (a). An edge between two nodes is drawn if two rings are topologically linked. Connected components are highlighted in different colors. (see text for details)

will consider both, symmetric and asymmetric, boxes. The former is investigated for simplicity, while the latter mimics the aspect ratio of a Kinetoplast disc, which is roughly 0.5 . The results from the two cases are also compared.

The internal (un-)linking action performed by topoisomerase enzymes is modelled by allowing the chains to pass through one-another (phantom loops), while retaining self-avoidance, for a time equal to the relaxation time of the chains $\tau_{R} \simeq$ $R_{g}^{2} / D_{R}$, with $R_{g}$ being the radius of gyration of the rings and $D_{R}$ their diffusion coefficient, both of which have been measured beforehand in dilute conditions. After this, the inter-chain steric interaction is turned on by pushing the beads apart via a soft potential of the form

$$
E_{s}(t)=A\left(0,50 ; 10^{5}\right)\left(1+\cos \left(\frac{\pi r}{r_{c}}\right)\right)
$$

with $A(x, y ; t)$ a ramp function which brings $A(x, y ; t)$ from $x$ to $y$ in $t$ timesteps and $r_{c}=2^{1 / 6} \sigma$.

By repeating this process over time, statistically independent system configurations can be sampled every $\tau_{R}$ timesteps. It is important to bear in mind that the ensemble of configurations thereby generated should be interpreted as a collection of independent equilibrated systems, rather than a dynamical sequence of network conformations over time. In other words, the networks generated by this process probe KDNA structures which may be thought of as coming from different cells.

The stage in which mini-circles are allowed to pass through one another mimics the presence of topological enzymes such as topoisomerases, which can either link or un-link the mini-circles from the neighbours. This in fact allows the mini-circles to freely diffuse while temporarily unlinked. As a reference, one should think to networks observed, for instance, when looking at the Kinetoplast at the end of the duplicating process. At this stage in fact, a simultaneous topological and structural 
re-arrangement of the network has to take place, involving both, topological enzymes and mass relaxation via mini-circles diffusion, which is itself allowed by the presence of topoisomerases. The observables in the system are then averaged over an ensemble formed by 5000 independent configurations.

\subsection{Probing the Network Topology}

In order to study the topological properties of the networks generated with the aforementioned method, the ensemble of configurations is mapped to an ensemble of graphs (see Fig. 5.3). This is done by identifying rings with nodes and topological linking with edges. The linking between pairs of rings is computed via the Gauss linking number

$$
L k(i, j)=\frac{1}{4 \pi} \int_{\gamma_{i}} \int_{\gamma_{j}} \frac{d \boldsymbol{r}_{i} \times d \boldsymbol{r}_{j}}{\left|\boldsymbol{r}_{i}-\boldsymbol{r}_{j}\right|^{3}}\left|\boldsymbol{r}_{i}-\boldsymbol{r}_{j}\right|
$$

where $\gamma_{i}$ and $\gamma_{j}$ are the contours of the two rings, and $\boldsymbol{r}_{i}$ and $\boldsymbol{r}_{j}$ the respective spatial coordinates Orlandini et al., 2000, Orlandini and Whittington, 2004. This is a topological invariant which describes the pairwise state of rings, as long as they are not allowed to pass through each other.

For each configuration in the ensemble, a corresponding network representation is generated by assigning an (undirected) edge between each two rings which have $L k(i, j) \neq 0$. This maps the network of linked rings to an undirected graph, whose properties are intimately related. Note that, because this procedure is based on the calculation of the pairwise linking number, it would classify Borromean and Brunnian links as unlinked; such non-trivial links are expected to be rare within the Kinetoplast network, where a good approximation is that each mini-circle is linked identically once to its neighbours Chen et al., 1995b.

The main control parameter is the size of the confining container, $L$, which is modified in order to vary the density $\rho$. This determines the physical properties of the resulting network. The overlapping (number) density $\rho^{*}$, at which rings start to feel each other, can be estimated as $\rho^{*}=M /\left(4 / 3 \pi R_{g}^{3}\right) \sim 0.0076 \sigma^{-3}$, where $R_{g}$ has been measured from relaxed rings in sparse solution. To convert this to a biologically realistic value, it can be assumed that the volume occupied by each bead is that of a cylinder of size and height equal to $\sigma$, which leads to a volume concentration $\phi^{*} \sim 0.60 \%$ occupied by the DNA, or equivalently a mass concentration $c^{*} \sim 8.1$ $\mathrm{mg} / \mathrm{ml}$ (calculated with a DNA mass density $\rho_{D N A}=1.35 \mathrm{~g} / \mathrm{cm}^{3}$ Matthews, 1968).

A good way of studying the properties of a graph $G$ is by investigating its first Betti number, $b_{1}(G)$, and its giant connected component, $G C C(G)$ (see Ch. 4). Recall that the former is defined as $b_{1}(G) \equiv N_{C C}-|\mathcal{V}|+|\mathcal{E}|$, where $N_{C C}$ is the number of connected components while $|\mathcal{V}|$ and $|\mathcal{E}|$ are the size of the sets of vertices and edges, respectively. The latter, $G C C(G)$ is the largest set of nodes for which 


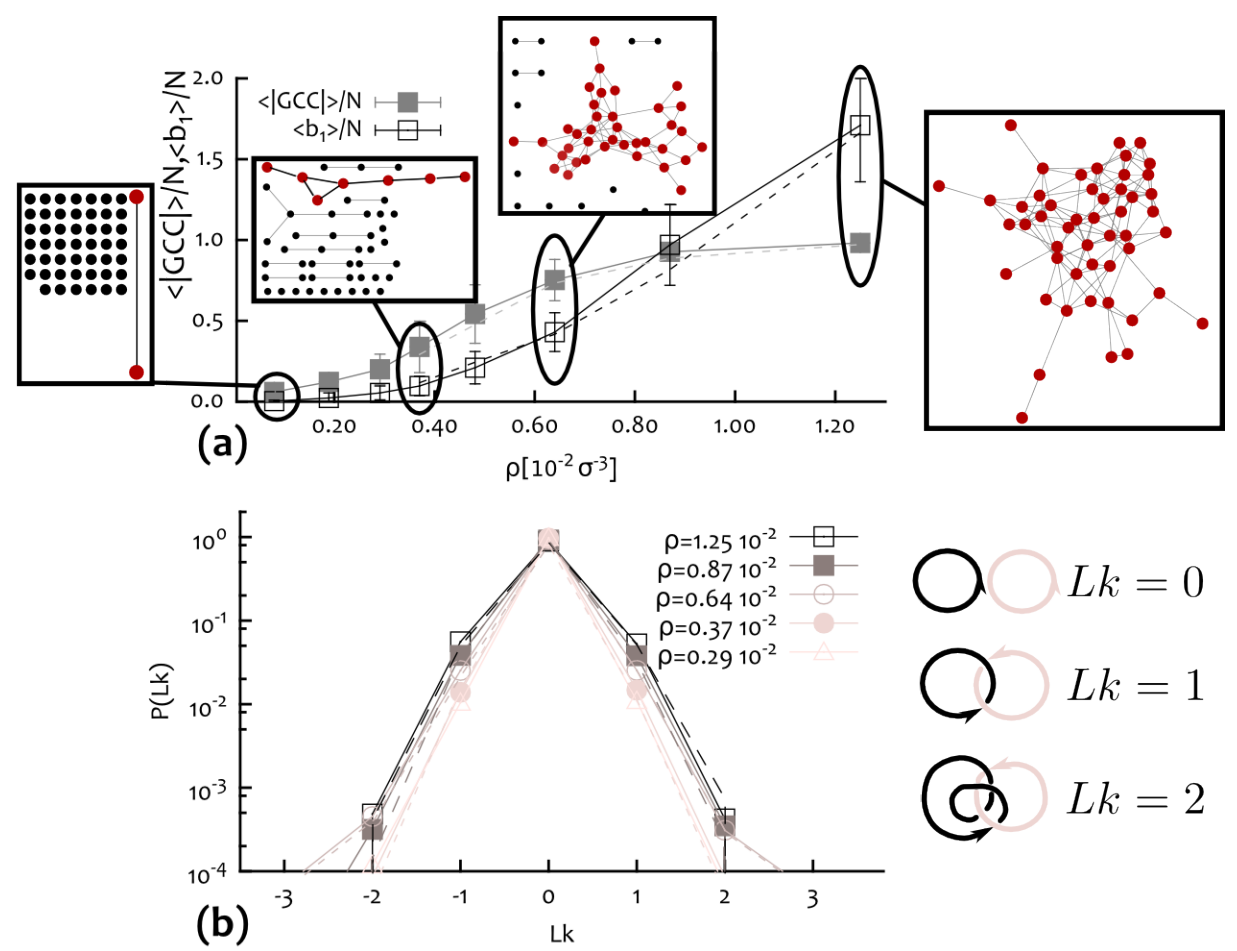

Figure 5.4: (a) Average fraction of nodes belonging to the giant connected component $\langle|G C C|\rangle$ (filled squares) and average first Betti number $\left\langle b_{1}\right\rangle$ (empty squares) as a function of the density $\rho$. Snapshots of the typical configurations are also shown, with the respective giant connected components highlighted in red. (b) Linking number distribution $p(L k)$ for different values of the density $\rho$. In agreement with experimental findings Chen et al., 1995b], linking numbers $|L k|>1$ are very rare. In both figures, dashed lines show the results obtained from simulations performed in asymmetric boxes (see text for details).

every node is connected to any other node by a sequence of vertices belonging to the set. For instance, in Fig. 5.3(b), the giant connected component corresponds to the red cluster.

Here I define a network to be "percolating" when the size of the giant connected component $|G C C|$ is of the same order as the number of nodes in the whole graph. The percolation density $\rho_{p}$ is then defined as the density above which the system shows a non-zero probability of percolation. The giant connected component of a percolating network is a spanning, or percolating, cluster. While the size of the $G C C$ gives some insight into the connectivity of the network, the first Betti number, $b_{1}(G)$, captures some topological features. In fact, $b_{1}(G)$ equals the number of closed subgraphs in the graph, which is also the total number of cyclic d-mer:* For instance, in the case of mostly unconnected graphs, $b_{1}(G) \simeq 0$, in the case of lattices it is $b_{1}(G) \simeq N$ while for nearly fully connected graphs where $|\mathcal{E}| \simeq N(N-1) / 2$, $b_{1}(G) \simeq N^{2} / 2$ for large $N$. An increase in $b_{1}(G)$ corresponds to both, an increase in network connectivity and an increase in cyclic structures.

Fig. 5.4(a) shows the average size of the giant connected component, $|G C C|$,

${ }^{*}$ Technically, it is the rank of the first homology group $H_{1}$ which measures the number of 1dimensional cycles in the graph. 
and the average first Betti number of the graph $G, b_{1}(G)$, divided by the size of the system $N$ as a function of the system density $\rho$. The plot suggests that a percolating component can be observed in the system for densities $\rho \gtrsim 0.0064 \sigma^{-3}$. Although the precise value of the percolation density is not well defined for finite systems, the model allows to predict the emergence of a state in which a fraction close to unity of rings in the system is topologically interlocked in a single cluster for values of the density above $\rho_{p}$. In addition, it is worth stressing that the value $\rho \simeq 0.0064$ $\sigma^{-3}$ has to be considered only as rough estimate of the true percolation density $\bar{\rho}_{p}$, whose estimation is beyond the scope of this work (see Ref. Diao et al., 2012 for more accurate estimations of $\bar{\rho}_{p}$ for a system of linked rings).

The distribution of linking number $p(L k)$, is shown in Fig. 5.4(b). This is found to be peaked at zero for any density $\rho$ investigated here, i.e. the networks are far from being fully connected and, in fact, most of the pairs are unlinked. One can also observe that the distribution is symmetric with respect to zero, i.e. positive linking is as frequent as negative linking. The "shoulders" of the distribution at $L k= \pm 1$ increase with $\rho$ although values of $|L k|>1$ are very unlikely $\left(<10^{-3}\right)$. This is once again in agreement with experimental findings Chen et al., 1995b, Jensen and Englund, 2012, which observed that each linked mini-circle is linked only once with its neighbours. This is presumably because the rings are relatively stiff $\left(L_{c} / l_{K} \sim 3.2\right.$ is comparable to that of mini-circles [Shapiro and Englund, 1995]), thereby suppressing configurations generating higher linking numbers. Note that, for simplicity, only one undirected edge is assigned between a pair of nodes even in those cases in which a higher linking number is found. Because they are so rare, they represent a small fraction of all the links, which can be neglected. The mean linking number $\langle L k\rangle$ is zero within errors, as configurations with $L k=-1$ are as likely as ones with $L k=+1$. Note that in this model, the rings have a canonical orientation given by the progressive number of the beads composing the polymers. For this reason, a configuration of a pair of rings with $L k=+1$ can be distinguished from one with $L k=-1$. Experimentally, although might be more difficult, the same can be done by establishing an orientation given by the genetic sequence encoded in the mini-circles.

In Fig. 5.5.(a) the valence, or mean vertex degree $\langle k\rangle$ is shown together with the linking probability $p_{L k}$ as a function of the density $\rho$. The figure shows that the average degree $\langle k\rangle$ scales linearly with the density $\rho$, in agreement with previous findings Diao et al., 2012. One can also see that at $\rho=\rho_{p}$, the valence of our network is around 3 (precisely, it is $\langle k\rangle=2.6 \pm 0.4$ ), a value compatible with that found in experiments in pre-replicating, or post-mitotic, networks Chen et al., 1995b, Chen et al., 1995a. By assuming that the network configurations are sampled from an ensemble of random graphs, the linking probability $p_{L k}$ can be calculated as

$$
p_{L k}=\langle k\rangle /(N-1) .
$$


The assumption that the network configurations can be described by random graphs is not justified a priori. On the the other hand, one can check that this assumption is valid by comparing the degree distribution $p(k)$ obtained from the simulations (see data points and dashed lines in Fig. 5.5(b)) and the random graph distribution $p_{\text {rg }}(k ; p)$ :

$$
p_{r g}\left(k ; p_{L k}\right)=\left(\begin{array}{c}
N-1 \\
k
\end{array}\right) p_{L k}^{k}\left(1-p_{L k}\right)^{N-1-k},
$$

with $p=p_{L k}$ obtained from Eq. 5.3 (see solid lines in Fig. 5.5). The theoretical prediction for random graphs with $p=p_{L k}$ and the data-points obtained from simulations are in good agreement, although the discrepancies between theoretical (mean field) prediction and the simulations might suggest that the use of an "effective" $p^{\prime} \neq p_{L k}$ might be more appropriate. A part from these small deviations, one can observe that the system can be thought of as a random graph with an edge probability $p \sim p_{L k}$ which is directly proportional to the valence of the rings and inversely proportional to the number of rings in the box.

The physical interpretation of this result in terms of the Kinetoplast structure is the following: the mini-circles are subject to thermal fluctuations inside the region of the mitochondrion which contains the Kinetoplast, and therefore, they are expected to diffuse meanwhile the network structure is re-arranging the topological structure thanks to the presence of topoisomerases. Because of this, it is reasonable to expect that the network of mini-circles would form a random arrangement of linked rings with a given valence. The valence has been experimentally found to be near 3 , and in the model presented here I find that this value of the valence is best retrieved for systems whose density is around $\rho_{p} \simeq 0.0064 \sigma^{-3}$.

It is worth noting that Figs. 5.4 and 5.5 also report the results obtained by simulating the system in asymmetric boxes. These are shown as dashed lines. The two cases are in very good qualitative agreement. Small deviations are found for the degree distributions and the average Betti number $\left\langle b_{1}\right\rangle$. This suggests that the shape of the confinement has little effect in the inner topological KDNA structure.

In Fig. 5.6(a)-(h), all the possible sub-graphs with three and four nodes are shown. These are here defined as "motifs". Every connected graph formed by three and four nodes is isomorphic to one of those pictured in Fig. 5.6.(a)-(b) and Fig. 5.3.(c)-(h), respectively. In Fig. 5.6, the relative frequency of motifs of each type is also shown. This is obtained by considering every connected sub-graph in a given graph and by checking whether isomorphic to one of the motifs. One can observe that linear trimers (motif in Fig. 5.6(a)) are much more frequent than cyclic ones (motif in Fig. 5.6(b)), for any density $\rho$. Similarly, linear tetramers are three times more common than branched tetramers (motif in Fig. 5.6(d)). Finally, fully connected cyclic tetramers are highly unlikely but seem to become more abundant with increasing values of $\rho$. Because the Kinetoplast network in vivo is not directly 

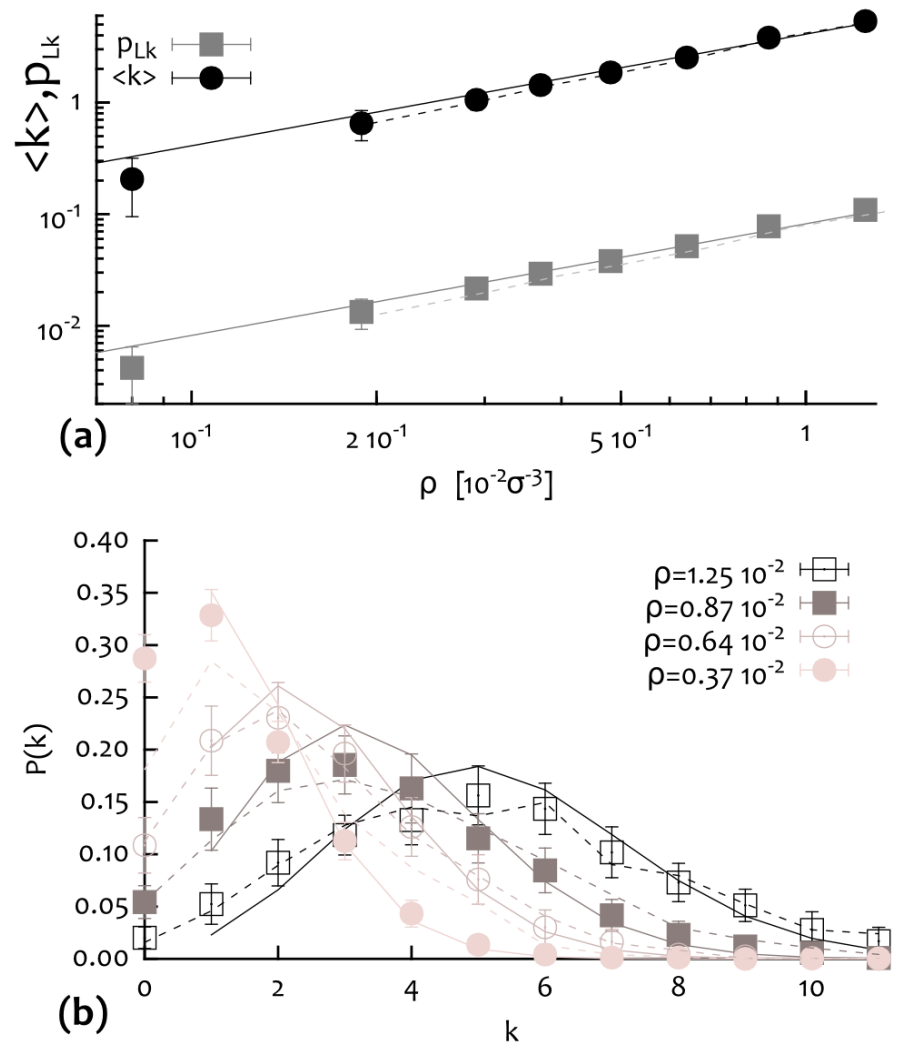

Figure 5.5: (a) Valence, or mean degree, of the nodes $\langle k\rangle$ together with the linking probability $p_{L k}$ (Eq. (5.3) ) as a function of the density $\rho$. Solid lines are linear fits, while the data-points are from simulations in symmetric boxes. The plot shows that they both linearly increase with $\rho$, in agreement with previous findings Diao et al., 2012. (b) Degree distribution $p(k)$. Data points are obtained from simulations in symmetric boxes, solid lines show the degree distributions of random graphs with edge probability $p=p_{L k}$ (Eq. (5.4)). In both figures, dashed lines show the results obtained from simulations performed in asymmetric boxes (see text for details).

accessible by experimental procedures, previous studies probed the network by using a method commonly called "digestion". This procedure and its in silico realisation are explored in the next section.

\subsection{An in silico Digestion}

Restriction nuclease enzymes are commonly added in solutions containing DNA in order to fragment long strands of genetic material into shorter pieces. These enzymes cut the sugar-phosphate backbone of the DNA at specific sites thereby generating shorter sequences which can be manipulated Alberts et al., 2014. This biological procedure is also known as "digestion" Roberts and Murray, 1976. To further quantitatively compare the properties and structure of the random network of linked confined loops found in the previous sections to those of the in vivo Kinetoplast DNA, I will here describe a procedure for the in silico network digestion, i.e. the 


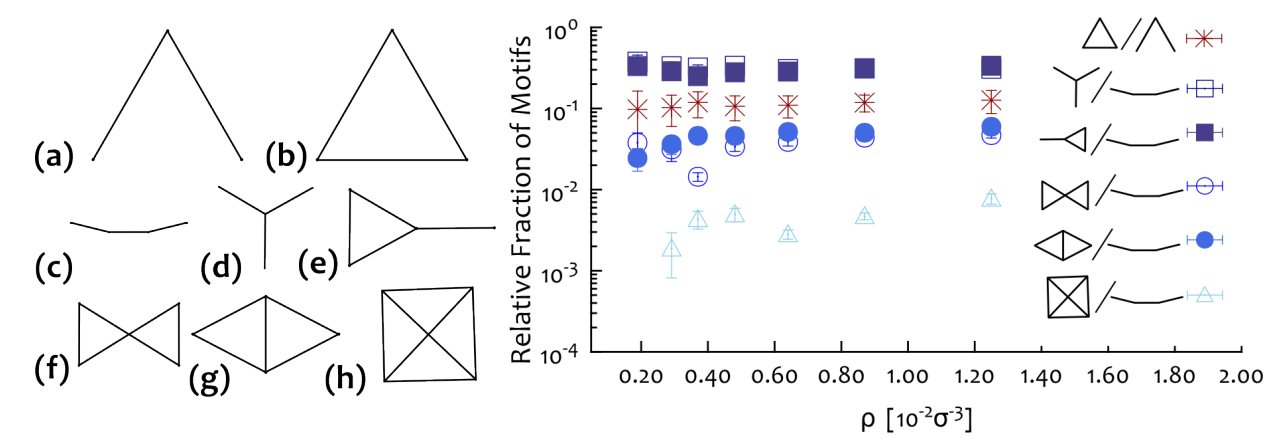

Figure 5.6: Showing the fraction of cyclic trimers (b) over linear trimers (a) (red) and the fraction of different tetramers $((\mathbf{d})-(\mathbf{h}))$ over linear tetramers (c) (shades of blue) as a function of the density $\rho$.

random breakage of the network due to a concentration of restriction enzymes. This method was used in particular in Chen et al., 1995b and Chen et al., 1995a to experimentally probe the KDNA topology in vitro.

To model digestion in silico, I associate a probability $p$ to each bead composing the rings to be removed. Such probability is related to the concentration of restriction enzymes in the solution and time left to act on DNA mini-circles. The equivalent probability $p_{r}$ of a ring to become linearised, e.g. by removing one or more of its beads, is

$$
p_{r}=1-(1-p)^{M} \sim M p
$$

where $M$ is the number of beads composing the rings. This procedure naturally maps to the graph representation as one can remove a node from the network with the same probability $p_{r}$.

In practice, I consider an ensemble of 5000 (independent) network configurations from the MD simulations and for each one of them I simulate 10 digestions by randomly removing nodes with probability $p_{r}$ from the corresponding graph. I then average the observables over the ensemble of $5010^{3}$ simulated digestions. The average number of removed nodes, or linearised mini-circles, is $\langle l\rangle=p_{r} N$ per network. At the end of the (partial) digestions I measure the fraction of monomers (single unlinked rings), dimers (two linked rings) and trimers (three linked rings) obtained from the digested network. Experimentally, these quantities can be probed by running a high resolution gel electrophoresis test on the digested KDNA networks, as done in Chen et al., 1995b. The relative fraction of monomers, dimers, trimers etc., correlates directly with the intensity of the bands, as these oligomers move with different speed when pulled through the gel (see also Ch. 6).

In Fig. 5.7(a)-(c) I report the findings for different values of density $\rho$, as a function of the linearised fraction of mini-circles, $\langle l\rangle$ (to ease comparison with the data in Chen et al., 1995b). In Fig. 5.7(d) I also show the probability of finding a cluster which contains more than half of the nodes of the initial network after the 

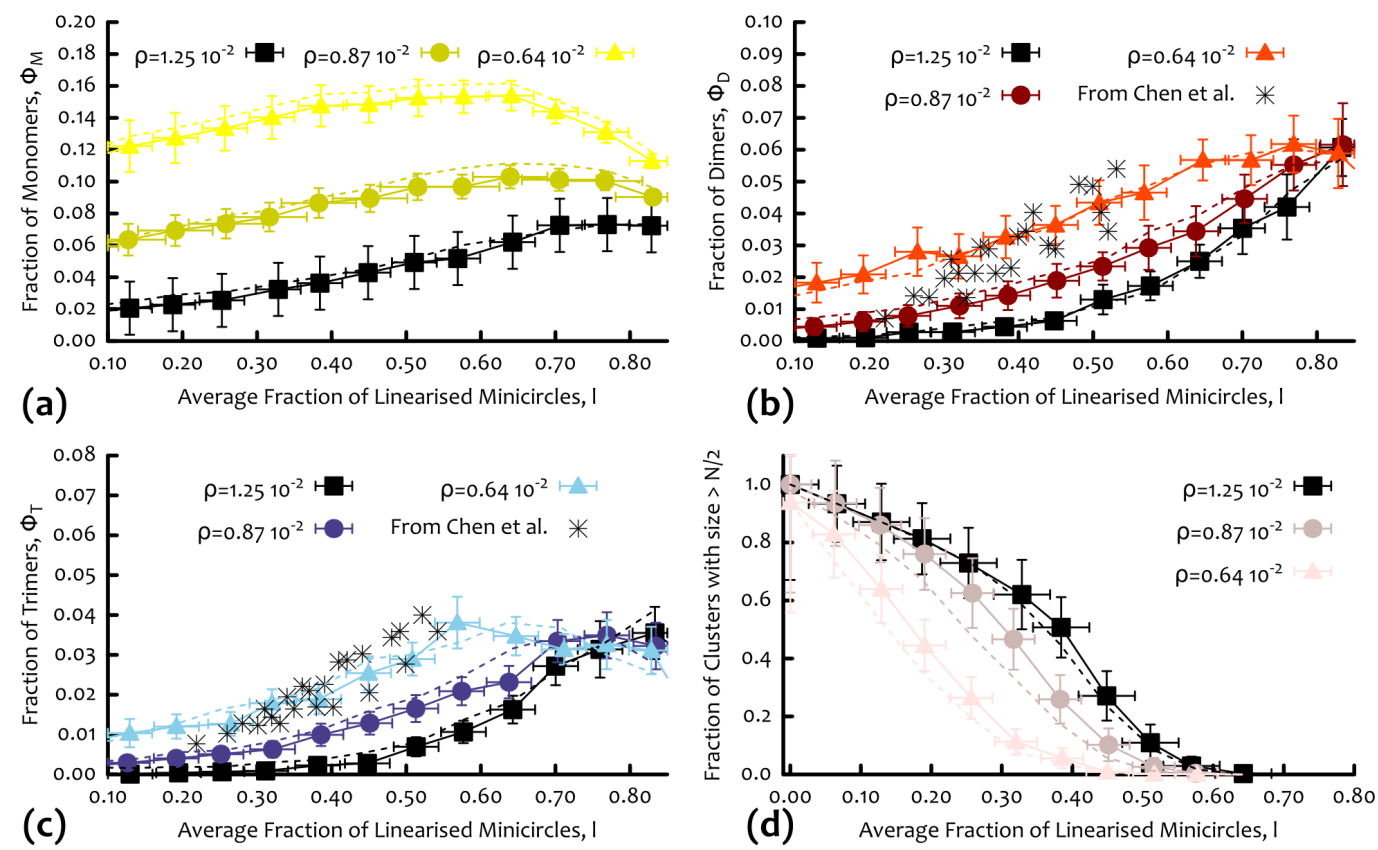

Figure 5.7: Fraction of (a) Monomers, (b) Dimers, (c) Trimers and (d) Clusters with size $s>N / 2$ as a function of the fraction of mini-circles linearised during the digestion, for different densities $\rho$. Crosses represent experimental data from Ref. Chen et al., 1995b. Dashed lines represent values obtained by performing simulations in asymmetric boxes. It is worth highlighting that while the average fraction of linearised mini-circles can be greater than 0.5 , the error on the value of $\langle l\rangle$ implies that the probability of finding a graph with a cluster of size $s>N / 2$ in the ensemble of all the digested graphs can be non-zero.

digestion.

Removing an increasing number of nodes from the networks results in an effective fragmentation which breaks the graphs into many isolated clusters. From this figure one can notice that the percolating networks $\left(\rho>0.0064 \sigma^{-3}\right)$ show high resistance against this random breakage. In fact, the probability of finding large clusters in the network does not decay to zero until almost half of the nodes, on average, has been removed. At this value one can still observe a little probability of finding clusters containing more than half of the nodes. Although this sounds counter-intuitive, one should bear in mind that the fraction of linearised mini-circle is an average value over many realisations and that the error on its value allows for the possibility of having digested clusters with $\langle l\rangle \gtrsim 0.5$ displaying some small probability of finding clusters with size $s>N / 2$. When viewed as a property of the biological Kinetoplast genome, this appears to be functionally relevant: the DNA network needs to remain intact when some of the mini-circles are removed, e.g. by topoisomerase II, either accidentally during the cell cycle, or during replication when decatenation is required.

The distributions of the fraction of monomers $\Phi_{M}$, dimers $\Phi_{D}$ and trimers $\Phi_{T}$ show peaks as a function of $\langle l\rangle$, whose locations depend on the density. In general, the value of $\rho$ at which the distributions reach their maximum increases with density, 
meaning that the denser the system, the more it has to be digested before the probability of observing monomers, dimers or trimers, rises and becomes sizeable. For fixed density, the peaks show that trimers are best produced at lower $\langle l\rangle$ than dimers, and dimers at lower $\langle l\rangle$ than monomers; this is expected as increasing $\langle l\rangle$ should increase the probability of finding smaller and smaller catenanes.

The best range of $\langle l\rangle$ within which gel electrophoresis of oligomers can give information on the network structure depends on the density $\rho$. For the highest density studied here, the fraction of linearised mini-circles has to be close to $80 \%$, while for $\rho \sim 0.0064 \sigma^{-3}$ the value of $\langle l\rangle$ can lay between $30 \%$ and $80 \%$, after which the survival fraction of dimers and trimers starts to decrease. This range is comparable to the one observed in Chen et al., 1995b. Even more strikingly, in Fig. 5.7 I superimpose the data from Chen et al., 1995b, and observe a striking quantitative agreement with the curve for $\rho=0.0064 \sigma^{-3}$ - one should at this point recall that $\rho=0.0064 \sigma^{-3}$ also leads to $\langle k\rangle \simeq 3$ as inferred from the experiments. Remarkably, considering the asymmetric system results in very little difference with the curves reported in Fig. 5.7. This strongly suggests that the simple symmetric confinement is enough to understand the Kinetoplast structure both qualitatively and quantitatively.

The fact that the Kinetoplast DNA is found to be geometrically similar to a quasi-two-dimensional disk may be due to a combination of the geometrical spatial confinement the network is subject to in vivo Lukeš et al., 2002, Ogbadoyi et al., 2003 Gluenz et al., 2007, Lai et al., 2008, Docampo et al., 2010, Diao et al., 2012 and of the action of histone-like DNA-binding proteins Hines and Ray, 1998, Avliyakulov et al., 2004. However, the findings shown here suggest that this feature is not essential to explain the existing digestion data. In addition, in Fig. 5.8. I qualitatively show that an ordered disc-like arrangement can be obtained from the performed simulations by adding attractive terms between the top and bottom walls of the confining box and some of the beads composing the rings. This mimics the presence of structural proteins which attach to both preferential binding sites, as bent DNA sequences Marini et al., 1982, Linial and Shlomai, 1988, and the mitochondrial envelope, thereby shaping the Kinetoplast network structure $X u$ and Ray, 1993, Xu et al., 1996, Silver et al., 1986, without changing the topological organisation. Disrupting the action of some histone-like proteins in C. fasciculata has in fact been observed to cause a rearrangement of the network while leaving the cell viability nearly unaffected [Lukěs et al., 2001]. All this encourages the conclusion that the geometrical arrangement occurs during at a later stage in the formation of the network, possibly to ease genes expression, without further affecting the inner topological structure.

One of the main conclusions that can be drawn is that the Kinetoplast topology is independent on the network packaging, while it is driven solely by geometrical confinement. This prediction can be tested, for instance, by measuring the valence 

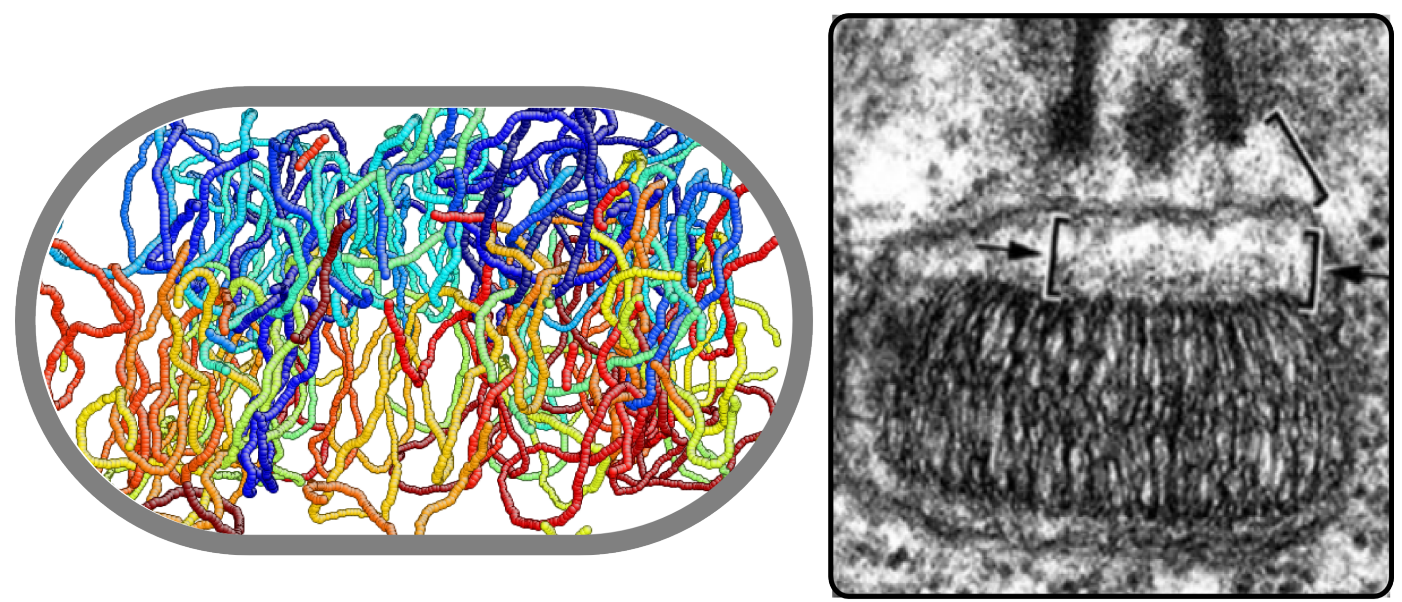

Figure 5.8: Snapshot of the simulated system in which a layered structure is achieved by taking into account the interactions of proteins with bent DNA sequences and mitochondrial envelope [Silver et al., 1986]. Here some of the beads belonging to the rings have attractive interactions with the top and bottom walls which are modelled via a standard LennardJones potential with cut-off $r_{c}=2.5 \sigma$. The rings are colored to help the visualisation of the layers. The layered structure is particularly reminiscent of the mini-circle arrangement in C. fasciculata well visible in Ogbadoyi et al., 2003.

of in vivo networks, as done in Chen et al., 1995b Chen et al., 1995a, formed when genes expressing KAP histone-like proteins are silenced, as done in Avliyakulov et al., 2004. The results presented here predict that in this case, the Kinetoplast should appear un-layered and disorganised, while retaining a valence near 3 . To my knowledge, there is no such type of experiments in the literature, and the results presented here might motivate such a study.

\subsection{A Linked Network of Rings at the Percolating Point}

Concluding, in this Chapter I examined the curious case of the Kinetoplast DNA, the mitochondrial genome of organisms of the class Kinetoplastida. I designed a model which allowed me to test some key features driving the Kinetoplast topological organisation. I found that by modelling the system as a collection of phantom loops and mapping the system to a graph of linked rings, one can observe that the onset of the percolation seem to occur at concentrations around $\rho=\rho_{p} \sim 0.0064$ $\sigma^{-3}$. This value should be interpreted as a rough estimate of the real percolation density $\bar{\rho}_{p}$. On the other hand, I observed that at around this monomer density, the mean valence of the nodes is found to be around 3, which is in agreement with the experimental findings in in vivo Kinetoplast DNA. Importantly, at the same density value, I compared the results from an in silico digestion of the network by a restriction enzyme, finding very good quantitative agreement with the experimental data found in Ref. Chen et al., 1995b. These results strongly suggest that the Kinetoplast topology can be understood as a system of confined linkable rings whose 
density $\rho$ can be tuned near the percolating density $\bar{\rho}_{p}$. In other words, it can be represented by a network of linked rings near its critical point, i.e. the point at which the network starts to show percolating behaviour.

The key message of this Chapter is that the available experimental findings can be understood in terms of a simpler system, where rings form linked structures by passing through one another and that a good agreement with the available experimental observations can be retrieved when the network is neither too poorly, nor too heavily, connected, but near what is seems to be the percolation threshold. The precise value of the latter, $\bar{\rho}_{p}$, is on the other hand, beyond the precision of the presented results. Nonetheless, it is possible to make some comments regarding this conceptual finding. In fact, being close to the percolation transition may well provide an evolutionary advantage for the Kinetoplast DNA network, as this structure may be favoured over a more heavily connected network, as it facilitates the decatenation during replication, but at the same time ensures that mini-circles are not released by mistake, conferring robustness and increasing the conservation of genetic material across generations. In other words, too heavily linked network would be very good at preserving the genetic material, but would severely slow down the replication stage of the network, where the Kinetoplast has to be taken apart. On the the other hand, networks that are too poorly linked might result in the loss of genetic material during cell mitosis and therefore put the cell viability at risk. Another property of the Kinetoplast-like network is that it is very resistant to digestion by a restriction enzyme, i.e. the digestion has to proceed significantly before large clusters disappear (see Fig. 5.7(d)). This feature again appears to be functionally relevant, as it provides a way to preserve genetic material against random breakage and replication mistakes. It is tempting to conjecture therefore, that the evolutionary pressure has pushed the KDNA toward a topological structure resembling a percolating network of linked rings at its transition, or critical, point, which offers many non-trivial evolutionary advantages.

A final remark is in order: these organisms have had a very unusual evolutionary path. They show a uniquely structured mitochondrial genome, very distant from anything else in Nature. On the other hand, they seem to have found an (unstable) equilibrium, since small changes in the Kinetoplast structure usually lead to the evolution of novel species which rapidly diverge from their common ancestor Lai et al., 2008 (in some cases, the network structure seem to have been traded for supercoiling, e.g. in the Pan-KDNA structure of Cryptobia helicis Lukeš et al., 2002]). I speculate that this equilibrium must have been reached via successive "attempts", i.e. mutations, which slightly modified the topological structure until a balance between several key elements, such as speed of replication (see previous Section and also Appendix C), accuracy of replication (Appendix C) and resistance against mistakes and network robustness (previous Section) had been reached, leading to the modern organisms of the class Kinetoplastida showing a KDNA. 
Finally, I would like to stress again that the survival of these organisms depends on the regulation of the Kinetoplast topology; Nonetheless, their genome is consistently duplicated with very few, or no, mistakes at every duplication cycle. Such reliable topology regulation is far from being trivial to achieve. During the '70s de Gennes advanced the idea of an "Olympic gel" de Gennes, 1979 and 40 years later, the scientific community has not made much progress toward its realisation. Perhaps the answer lies within these organisms, as they have made an Olympic gel out of their own mitochondrial genome (it is fair although to say that they had some millions of years to try and make it!).

A better understanding of how they manage this incredible feat would surely improve the current understanding and ability to realise similar topological materials, perhaps using techniques drawn from modern synthetic biology. 
The Red Queen said:

"Now, here, you see, it takes all the running

you can do, to keep in the same place"

L. Carroll

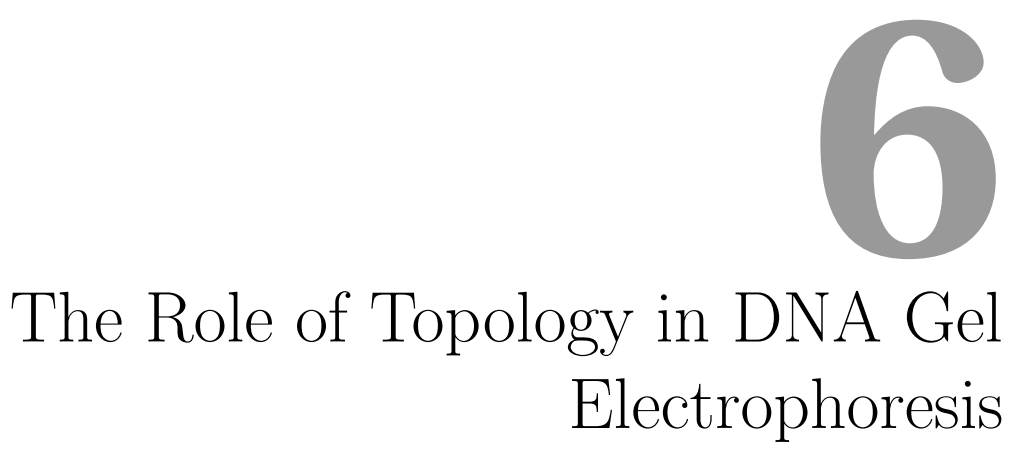

\section{Contents}

6.1 Gel Electrophoresis of DNA Rings and Strands . . . . 89

6.1.1 Getting More from Pushing Less . . . . . . . . . . . . . 90

6.1.2 Non-Equilibrium Response Theory . . . . . . . . . . . . . 92

6.1.3 Topology can Sense Disorder . . . . . . . . . . . . . . 95

6.2 Gel Electrophoresis of DNA Knots . . . . . . . . . 95

6.2.1 Non-monotonic Speed of DNA Knots in Gel . . . . . . . . . 97

6.2.2 Entanglement with Dangling Ends . . . . . . . . . . . . 999

6.2.3 An Equivalent Random Walk Description . . . . . . . . . 103

6.3 Conclusions . . . . . . . . . . . . . 105

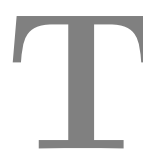

OPOLOGY plays a key role in the biophysics of DNA, and is intimately related to its functioning. For instance, transcription of a gene redistributes twist locally to create what is known as supercoiling, while catenanes or knots can prevent cell division, hence they need to be quickly and accurately removed by specialised enzymes known as topoisomerases. But how can one establish experimentally the topological state of a given DNA molecule? By far the most successful and widely used technique for this is gel electrophoresis Calladine et al., 1997. Bates and Maxwell, 2005. This method exploits the empirical observation that the mobility of a charged DNA molecule under an electric field and moving through a gel depends on its size, shape and topology Bates and Maxwell, 2005, Stasiak et al., 1996. Nowadays, gel electrophoresis is a ubiquitous technique Calladine et al., 1997, Viovy, 2000, Dorfman, 2010, since it readily allows the separation of polymers with different physical properties and it is systematically used for DNA identification and purification Calladine et al., 1997.

Gel electrophoresis is so empirically reliable that it can be used, for instance, to 
map replication origins and stalled replication forks Olavarrieta et al., 2002, to separate plasmids with different amount of supercoiling Olavarrieta et al., 2002 Cebrián et al., 2014, and to identify DNA knots Stasiak et al., 1996, Arsuaga et al., 2005. The most widely employed variant of this technique nowadays is two-dimensional gel electrophoresis, where a DNA molecule is subjected to a sequence of two fields, applied along orthogonal directions Bates and Maxwell, 2005]. The two runs are characterised by different field strengths, and sometimes also gel concentrations Cebrián et al., 2014; ; with suitable choices, the joint responses leads to increased sensitivity.

Even though widely employed, gel electrophoresis of topologically non-trivial polymers present some difficult theoretical challenges and remain largely theoretically unexplained. Some other aspects are reasonably well established. For instance, it is now widely accepted that the physics of the size-dependent migration of linear polymers can be explained by the theory of biased polymer reptation Rubinstein, 1987, Duke, 1989 Viovy and Duke, 1993 Barkema et al., 1994 Viovy, 2000. Likewise, the behaviour of, for example, nicked, torsionally relaxed, DNA knots in a sparse gel and under a weak field is analogous to that of molecules sedimenting under gravity Weber et al., 2013, Piili et al., 2013. The terminal velocity can be estimated via a balance between the applied force and the frictional opposing force, which is proportional to the average size of the molecule: as a result, more complex knots, which are smaller, move faster under the field. However, the mechanisms regulating the electrophoretic mobility of DNA knots at intermediate fields, and in more concentrated agar gels, are much less well understood Viovy, 2000, Weber et al., 2006b, Cebrián et al., 2014.

In these cases, experiments suggest that the mobility of DNA knots is usually a non-monotonic function of the knot complexity, or, more precisely, of their average crossing number Katritch et al., 1996b, Stasiak et al., 1996 (ACN): initially knots move more slowly as their ACN increases, while, past a critical ACN, more complex knots move faster. The combination of the responses to external fields directed along two perpendicular directions leads to a characteristic electrophoretic arc which allows one to separate the first simple knots more clearly in a 2D slab Trigueros et al., 2001, Arsuaga et al., 2002, Arsuaga et al., 2005, Cebrián et al., 2014. There is currently no theoretical framework that quantitatively explains the non-monotonic behaviour at intermediate or large fields and the consequent formation of arc patterns.

In this Chapter I will show that much of the difficulty in capturing their behaviour may be due to an incorrect model for the gel structure. In the past, the standard way to tackle this problem was to treat the gels as perfect meshes of obstacles Calladine et al., 1991, Alon and Mukamel, 1997, Viovy, 2000. On the other hand, it is well known that physical gels have irregularities, such as dangling ends Whytock and Finch, 1991. Cole and Akerman, 2003, Rahong et al., 2014. These are common in agarose gels formed at low agarose concentrations because many of the agarose bundles fail to cross-link with other fibers, thereby generating partially 
cross-linked open strands Whytock and Finch, 1991. More recently, dangling ends have also been directly observed in artificial gels made of solid nano-wires using transmission electron microscopy Rahong et al., 2014] (see also Ch. 3 for details on gels and their modelling).

The presence of these dangling ends plays a very weak role when linear polymers are undergoing gel electrophoresis. Conversely, gel electrophoresis experiments involving polymers with looped structures are expected to depend rather strongly on the topological interactions between the polymers and the gel structure (see for instance Fig. 6.1). When a dangling end threads through the ring polymer, the latter becomes "impaled" and its free motion is re-established only when the threading is removed. This makes the presence of dangling ends in the gel a crucial element for any realistic model aiming to faithfully describe gel electrophoresis dynamics of polymers with an architecture that includes one or more closed loops.

I will therefore focus on the dynamical properties of rings subject to an external force that move within a mesh of obstacles modelled as a $3 \mathrm{D}$ irregular cubic lattice. The "irregularities" are represented by randomly positioned dangling ends (see Fig. 3.5 in Ch. 3 or Fig. 6.1 in the next Section). These defects interact with the rings and these interactions may give rise to striking, counter-intuitive behaviour.

In Sec. 6.1 I will show that, in the regime of strong electric field and sufficiently high concentration of dangling ends, un-knotted ring polymers migrate slower as the external bias (force) is further increased. This means that the system displays a "negative differential mobility" Zia et al., 2002, Baerts et al., 2013, Ghosh et al., 2014, which has been beautifully captured by the phrase "getting more from pushing less" in the literature. In other words: the flux generated by large external fields is smaller than the one produced by weaker ones and in the system studied in this Chapter, this phenomenon is entirely due to the topology of the polymers.

I will also show that the topological interactions between ring polymers and the gel architecture can provide information on the microscopic structure of the gel. The results presented in fact suggest that a gel electrophoresis experiment can establish the level of disorder in the medium by comparing the results obtained by running linear and ring polymers. This represents a novel way to exploit topology to "sense" the disorder in the microscopic structure of a material in a non-invasive way.

Finally, in Sec. 6.2 I will investigate the gel electrophoresis of knotted ring polymers and, in particular, I will describe a model that captures the electrophoretic arc (i.e. non monotonic mobility) in $2 \mathrm{D}$ gel electrophoresis techniques. This will be explained in terms of competition between shrinking size and increasing complexity of DNA knots. 


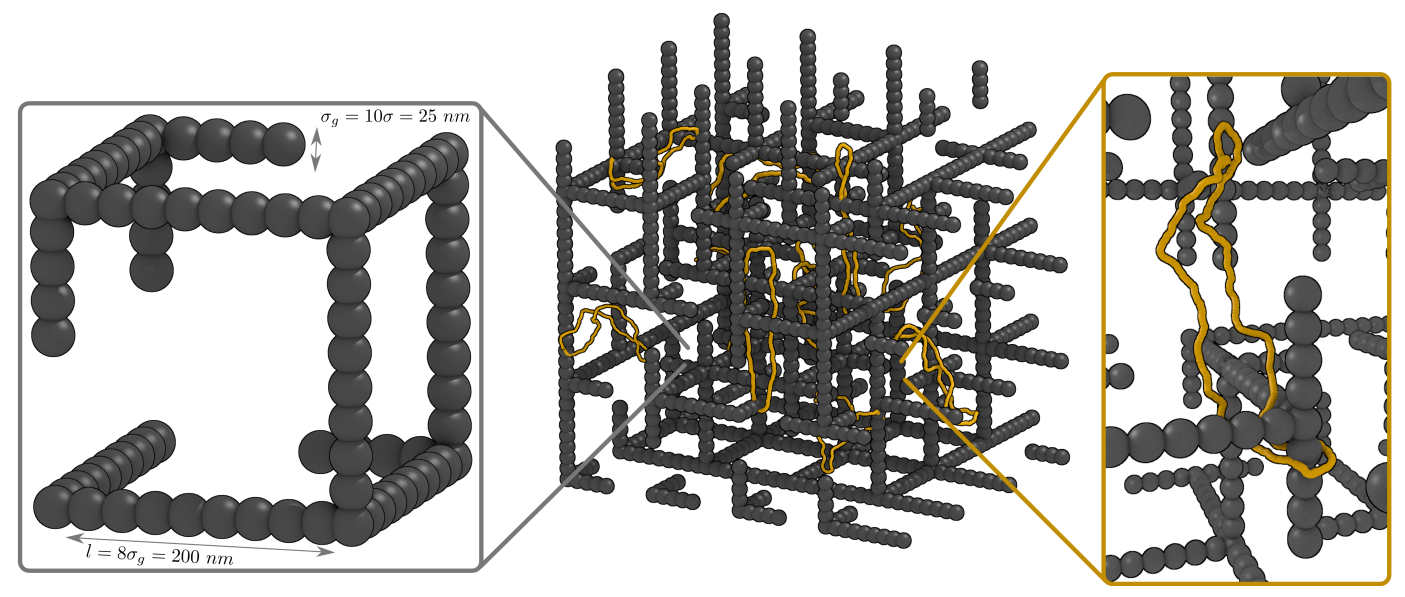

Figure 6.1: Assembling a random environment: With probability $p$ each edge is modified to have half edge knocked out at a random end. By assembling multiple cells we construct a random environment which resembles the disordered structure of a gel, where dangling ends populate the medium. To the far right we show the case where a ring is impaled by a dangling end. The external force in this case is directed upward. Periodic boundary conditions are applied to the simulation box. The gel structure is here thinned for clarity.

\subsection{Gel Electrophoresis of DNA Rings and Strands}

The random gel through which the rings move is modelled as a regular cubic lattice in which a fraction $p$ of dangling ends is produced by halving randomly the edges of the cubic lattice with probability $p^{\text {* }}$ (see Ch. 3). A typical configuration of this random mesh is reported in Fig. 6.1. By tuning the value of $p$, random meshes with different average number of dangling ends can be obtained. The lattice spacing of the gel is $200 \mathrm{~nm}$. The mesh of the gel is here constructed as a cubic lattice made of static beads of diameter $\sigma_{g}=10 \sigma$, where $\sigma$ is the size of the beads forming the polymer chains.

Rings moving through the gel are modelled by a set of $N$ circular worm-like chains each of $M$ beads of diameter $\sigma=2.5 \mathrm{~nm}$ (see Ch. 3 for details). Here we consider either systems of $N=10$ rings with $M=512$ beads each or systems of $N=20$ rings and $M=256$ beads. These two cases correspond to circular DNA of about $3.7 \mathrm{kbp}$ (contour length $\left.L_{c} \simeq 1.3 \mu \mathrm{m}\right)$ and $1.9 \mathrm{kbp}\left(L_{c} \simeq 0.65 \mu \mathrm{m}\right)$ respectively. The whole system is contained within a box of linear dimension $L=320 \sigma$ and has periodic boundary conditions in all three directions. In both cases, the system number density is $\rho \simeq N M /\left(L^{3}-V_{\text {gel }}(p)\right) \lesssim 1.6 \cdot 10^{-4} \sigma^{-3}$, much smaller than the value at which the chains start to overlap $\left(\rho^{*}=33.3 \cdot 10^{-4} \sigma^{-3}\right)$. This means that the systems are in the dilute limit. Interactions between rings are therefore neglected in the analytic calculations, although they do occur (rarely) in the simulations.

The external electric field is modelled as a constant force applied on each mono-

${ }^{*}$ The halving probability $p$ is always taken smaller than the critical (inverse) percolation probability $1-p_{c} \simeq 0.75$ ( $p_{c}$ being the bond percolation probability on a cubic lattice) to avoid the presence of sparse un-connected clusters of un-physically rigid parts of the gel. 

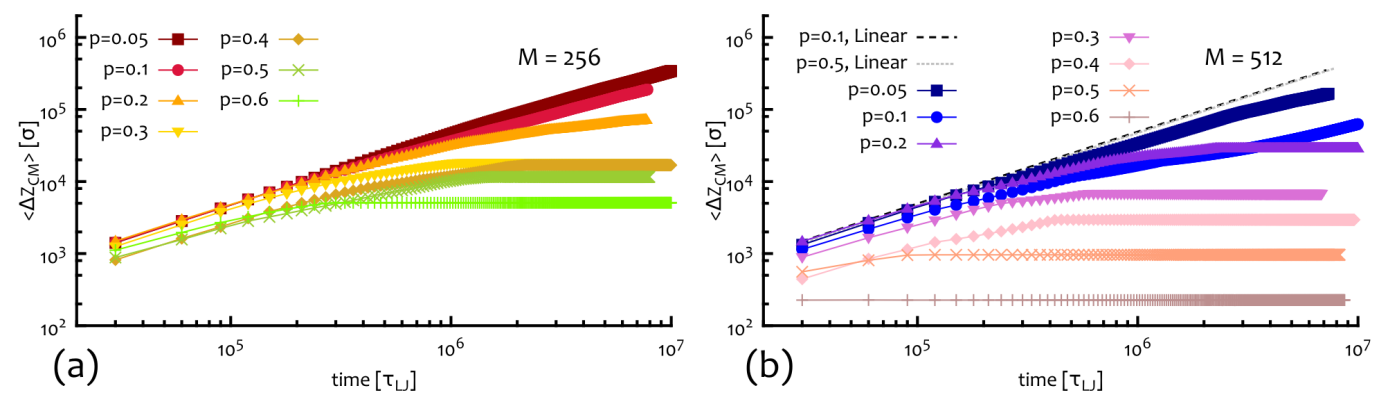

Figure 6.2: Time dependence of the average centre of mass displacement along the $z$ direction, $\left\langle\Delta Z_{C M}\right\rangle$, of rings inside the random mesh. The external field strength is $E=0.05$ $\epsilon / q \sigma$ and the two sets of rings considered have (a) $M=256$ and (b) $M=512$ beads. Different curves refer to different values of $p$. Results for linear polymers with $M=512$ and two different values of $p$ are reported as dashed black and dotted grey lines in (b). In system units $10^{6} \sigma \simeq 2.5 \mathrm{~mm}$ and $10^{7} \tau_{L J} \simeq 0.37 \mathrm{~s}$.

mer. Assuming that the electric charge is uniformly distributed along the rings, the total force $\boldsymbol{F}$ can be distributed uniformly on each monomer, which is subjected to a force $\boldsymbol{F} / M$. The force acting on each ring can be thought of as resulting from an electric field as $\boldsymbol{F}=q_{r} \boldsymbol{E}$, where $q_{r}=M q$ and $q$ is the representative charge of a single bead. The force acting on each bead can be expressed in units of $\epsilon / \sigma \simeq 1.6$ $p N$, the total force acting on the rings being a multiple of $F=1.6 M p N$. Since each bead corresponds to $\sigma=2.5 \mathrm{~nm} \simeq 7 \mathrm{bp}$, and each base-pair contains two phosphate groups which account for a negative charge each, one can approximate the charge in each bead as $14 q_{e}$, where $q_{e}$ is the electron charge

The force applied to the beads can therefore be thought of as a result of the action of an external electric field pointed towards $-\hat{z}$ and re-scaled by the charge of a bead. This means that the field strength applied to each bead can be expressed in units of $V / \mathrm{cm}$ as $\tilde{E}=1.6 / 22.4310^{19} \mathrm{pN} / \mathrm{C} \simeq 7 \mathrm{kV} / \mathrm{cm}$. In this Chapter, the fields used range from $10^{-3}$ to $10^{-1} \tilde{E}$, i.e. between 7 and $700 \mathrm{~V} / \mathrm{cm}$, which are roughly compatible to the values used in standard DNA gel electrophoresis Mickel et al., 1977, Levene and Zimm, 1987, Viovy, 2000.

\subsubsection{Getting More from Pushing Less}

To address the effect that the presence of dangling ends has on the dynamics of charged rings in the presence of an external electric field $\boldsymbol{E}$, it is useful to monitor the average centre of mass displacement along the direction $\hat{z}$ of the field, $\left\langle\Delta Z_{C M}\right\rangle$ for different values of the parameter $p$ (see Fig. 6.2). It is apparent that the rings significantly slow down as the fraction $p$ of incomplete edges (dangling ends) increases. To confirm that this is due to the ring topology, the same simulation is repeated

\footnotetext{
${ }^{\dagger}$ Here, I neglect the screening due to ions in solution Maffeo et al., 2010, Di Stefano et al., 2014. These will be considered in the next section. In any case, the correction due to the presence of ions account only for a pre-factor on the corresponding real-life values of the fields used in the simulations.
} 


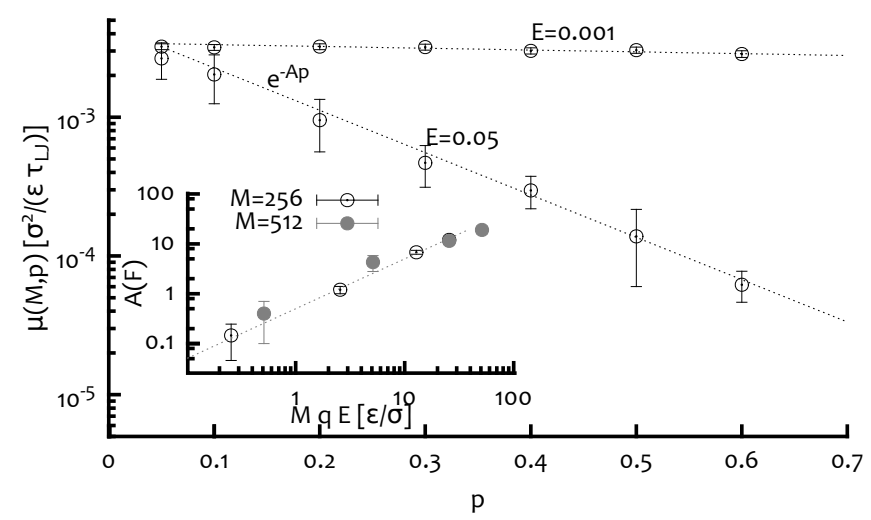

Figure 6.3: Rings mobility $\mu$, as defined in eq. 6.1), as a function of the average fraction of dangling ends $p$. The rings considered have $M=256$ and the two sets of symbols refer to two values of the field strength $E$. The data are well fitted by exponentials of the form $\mu \sim e^{-A p}$ (dotted lines). In the inset, the pre-factor $A$ is shown to scale extensively with $F=M q E$ (see text).

for linear chains with same contour lengths (see black dashed and grey dotted lines in Fig. 6.2): as expected the dangling ends do not interfere with the motion of the linear chains which are insensitive to changes in the microscopic structure of the gel (i.e. changes in $p$ ).

From the data in Fig. 6.2, it is possible to estimate the rings mobility $\mu(M, p)$ as a function of rings size and probability of forming a dangling end $p$, using the relation

$$
\mu(M, p)=\frac{\langle v\rangle}{|\boldsymbol{F}|}
$$

where the average velocity $\langle v\rangle$ is computed from the long time limit of the centre of mass displacement, namely

$$
\langle v\rangle=\lim _{t \rightarrow \infty} \frac{\left\langle\Delta Z_{C M}(t)\right\rangle}{t} .
$$

In the main panel of Fig. 6.3 the mobility $\mu(M, p)$ is reported for rings of $M=256$ beads driven by two different external fields strengths $|\boldsymbol{E}|=E$, as a function of the average fraction of dangling ends $p$. As expected, for fixed $E$ and $M$, the mobility decreases as $p$ increases since rings will be more often impaled by the dangling ends. This behaviour is apparently well captured by an exponential law of the form $\mu \sim e^{-A p}$, where $A$ seems to scale extensively with $M q E$ (inset of Fig. 6.3). One can interpret this result within the assumption that the rings move with mobility $\mu_{0}$ only when not impaled and otherwise are essentially immobile. Hence

$$
\mu=(1-s) \mu_{0}
$$

with $s$ the fraction of time in which a ring is stuck. This may be approximated by $s=\alpha p v /\left(\alpha p v+e^{-\Delta G / k_{B} T}\right)$, where the rate of hitting a dangling end in the 

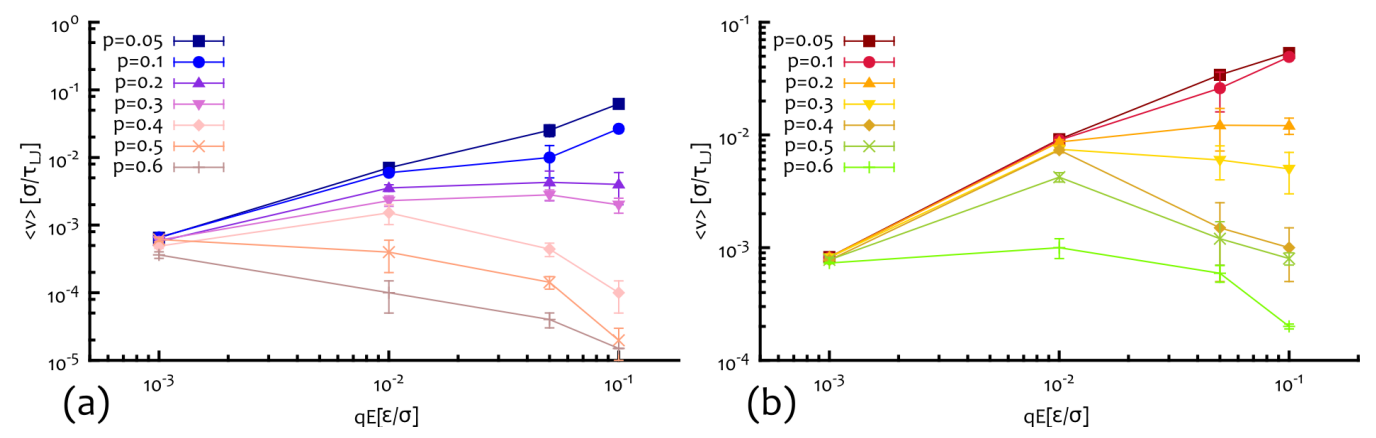

Figure 6.4: The average speed $\langle v\rangle$ of ring polymers as a function of the force on the single beads $q E$ for rings with, (a) $M=256$ and (b) $M=512$ beads, is shown. Notice the clear negative differential mobility $\partial\langle v\rangle / \partial E$ for fields $E>0.01$ and $p>0.3$. In system units 0.1 $\sigma / \tau_{L J} \simeq 6.7 \mathrm{~mm} / \mathrm{s}$ and $0.01 \epsilon / q \sigma \simeq 70 \mathrm{~V} / \mathrm{cm}$.

mobile state is proportional to both the velocity $v$ and the density of ends $p$ and the disentanglement rate is Arrhenius-like and proportional to $e^{-\Delta G / k_{B} T}$ with $\Delta G$ the relevant free energy barrier for disengagement from a dangling end. The fluctuation of energy $\Delta G$ required to disentangle from an impaled situation may be expected to have Arrhenius form $\Delta G=M q E l / 2$ where the ring must move the length of the penetrating segment $l / 2$ against a force $F=M q E$. For strong fields this captures the exponential variation with $A \sim M q E$ but not the exponential variation with $p$. Although we do not have a simple explanation for the latter, this might be related to the higher order structure of ramified ring polymers, or to multiple simultaneous interactions of the rings with dangling ends, both of which are completely neglected in our analysis.

Fig. 6.4 reveals the striking non-equilibrium property that the average centre of mass speed $\langle v\rangle$ decreases as the strength of the field $E$ increases for sufficiently high density of dangling ends $p$. This qualitative behaviour can be related to the definition of the mobility in eq. (6.1) combined with the empirical result of Fig. 6.3 that the mobility decreases with force. The average velocity might then be expected to scale as $\langle v\rangle \sim F e^{-c p F}$, with $c$ a constant, which has a maximum at an intermediate value of $F$.

\subsubsection{Non-Equilibrium Response Theory}

According to previous studies on the the linear response of non-equilibrium systems [Baiesi et al., 2009, Baiesi et al., 2011, Baerts et al., 2013], it is useful to probe the differential mobility of the rings, which can be computed numerically as:

$$
\mu_{D}^{N}=\frac{\partial}{\partial F}\langle v(F)\rangle
$$

This is the long time limit of the response of the mean velocity to a change in the field strength $E$. In Appendix DI show that this quantity can also take the following 
form (the superscript $A$ indicates that this is obtained in a different way with respect to the "numerical" differential mobility $\left.\mu_{D}^{N}\right)$ :

$$
\mu_{D}^{A}=\lim _{t \rightarrow \infty} \frac{\mathcal{D}(t)-\mathcal{C}(t)}{k_{B} T}
$$

where the first term

$$
\mathcal{D}(t)=\frac{1}{2 t}\left[\left\langle\Delta z^{2}(t)\right\rangle-\langle\Delta z(t)\rangle^{2}\right]
$$

is the non-equilibrium generalisation of the $1 \mathrm{D}$ diffusion constant, and the second term is

$$
\mathcal{C}(t)=\frac{1}{2 t}\left[\left\langle\int_{0}^{t} d s \nu \Psi(s) \Delta z(t)\right\rangle-\left\langle\int_{0}^{t} d s \frac{\Psi(s)}{\xi}\right\rangle\langle\Delta z(t)\rangle\right]
$$

where $\xi$ is the friction acting on each polymer bead, $\Delta z(t)$ is the displacement of a bead at time $t$ along $\hat{z}$ and $\Psi(s)=-\partial U(s) / \partial z+F$ is the sum of all the forces projected on $\hat{z}$ acting on a bead at time $s$ Baiesi et al., 2009, Baiesi et al., 2011. This covariance between time-averaged forces and displacements appears because the rings are pushed significantly far from equilibrium via an external force $F=M q E$. Clearly at equilibrium detailed balance holds, the displacements are on average uncorrelated with the force, $\mathcal{C}(t)$ would be zero and the fluctuationdissipation theorem would be valid according to the usual form of the Einstein relation $\mu=\mathcal{D} / k_{B} T$.

It is interesting to notice that the presence of the non-equilibrium term $\mathcal{C}$ may introduce novel features into the system. In particular, from Eq. 6.5), it is apparent that if $\mathcal{C}>\mathcal{D}$ the differential mobility becomes negative. This is an exclusive aspect of non-equilibrium systems. In order to compute $\mu_{D}^{A}$, I first reach a steady state with constant average velocity at a fixed $E$ and then I slightly increase the field strength. Consequently, I monitor the position and the sum of all the forces acting on the beads at each time-step until a new steady state is reached. The average is then performed over the beads belonging to a ring and over all the rings of the system.

The "numerical" differential mobility $\mu_{D}^{N}$ can instead be straightforwardly computed by taking the differential of the velocity with respect to the applied force, i.e. through a discrete version of eq. (6.4):

$$
\mu_{D}^{N} \equiv \frac{\Delta\langle v\rangle}{\Delta F} .
$$

To evaluate this derivative, one can make use of the average speed $\langle v\rangle$ computed in Fig. 6.4 and Eq. 6.4). In Fig. 6.5(a) I show the results obtained via these two different approaches, yellow/orange data points indicate $\mu_{D}^{N}$ while the yellow shaded area indicates $\mu_{D}^{A}$, for the case of a system with rings with length $M=256$ beads and a gel with $p=0.4$. As one can notice, the agreement is very good for the whole range of the electric fields considered. 

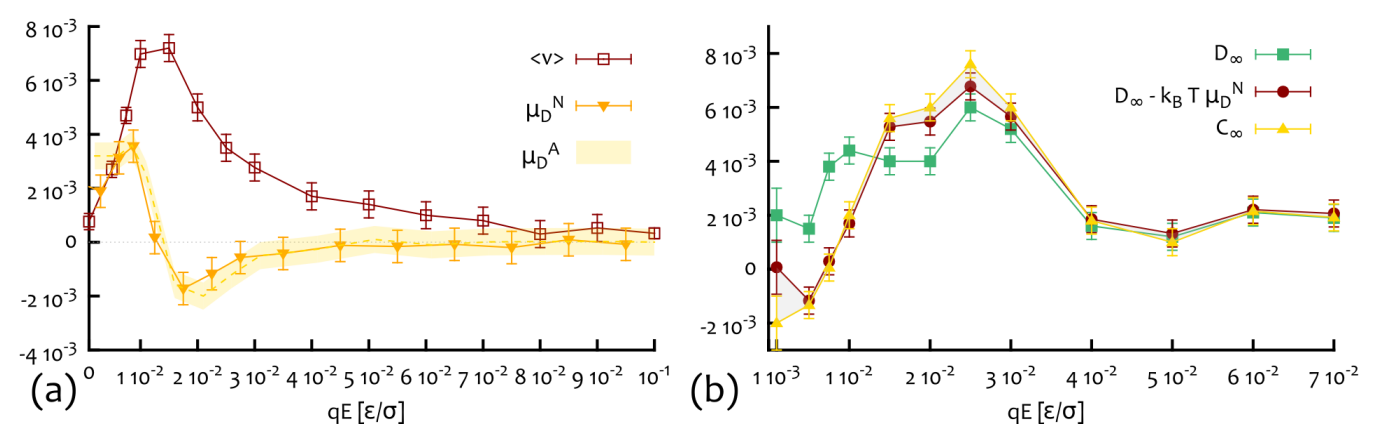

Figure 6.5: (a) Average speed of the centre of mass of rings, $\langle v\rangle$ in units of $\sigma / \tau_{L J}$, for a system of $N=20$ rings with $M=256$ beads each and with $p=0.4$ (dark-yellow diamonds in Fig. 6.4. The differential mobilities computed as in eq. 6.4 $\mu_{D}^{N}$ and as in eq. 6.5) $\mu_{D}^{A}$, in units of $\sigma^{2} / \epsilon \tau_{L J}$, as a function of the force acting on single beads $q E$ are shown to be in very good agreement. In system units the minimum of the differential mobility is reached at field strength around $0.015 \epsilon / q \sigma \simeq 100 \mathrm{~V} / \mathrm{cm}$.(b)The long time limit of the terms $\mathcal{D}$ (green), $\mathcal{D}-k_{B} T \mu_{D}^{N}$ (red) and $\mathcal{C}$ (yellow) are plotted against the force acting on single beads $q E$. While $\mathcal{D}$ and $\mu_{D}^{N}$ can be easily measured during an experiment Mickel et al., 1977, the latter $(\mathcal{C})$ can be retrieved by making use of eq. 6.5 and Fig. 6.5 a), i.e. $\mathcal{C}=\mathcal{D}-k_{B} T \mu_{D}^{N}$. The shaded area highlights the small difference between the predicted value $\left(\mathcal{D}-k_{B} T \mu_{D}^{N}\right)$ and the measured value of $\mathcal{C}$.

It is important to notice that while tracking the position of particles travelling through a medium is a common experimental procedure, to get information on the forces acting on the particles is experimentally very difficult if not impossible. On the other hand, according to Eq. (6.5) and Fig. 6.5(a), if one knew $\mu_{D}^{N}$ and $\mathcal{D}$ from the experiments it would be possible to estimate $\mathcal{C}$ as Bohec et al., 2013

$$
\mathcal{C}=\mathcal{D}-k_{B} T \mu_{D}^{A}=\mathcal{D}-k_{B} T \mu_{D}^{N}
$$

Since $\mathcal{C}$ is the covariance between a total displacement and an average force (in the $z$ direction), its value gives some information about the force experienced by a bead.

To show that the indirect determination of $\mathcal{C}$ in experiments is a viable strategy, in Fig. 6.5(b) I compare the long time limit values of $\mathcal{D}-k_{B} T \mu_{D}^{N}$ with values of $\mathcal{C}$ known directly from simulations, finding again a very good agreement. There is only a slight discrepancy between the $\mathcal{C}$ and $\mathcal{D}-k_{B} T \mu_{D}^{N}$ at very low fields, which is most likely due to errors in the measurements that become noisier at low fields, where diffusion dominates.

Note that by construction the generalized diffusion constant $\mathcal{D}$ is greater than zero, also when out of equilibrium Baiesi et al., 2011] (see Fig. 6.5(b)). Conversely, one may find negative covariances $\mathcal{C}$. Fig. 6.5(b) suggests that this might happen for weak fields $E \gtrsim 0$. By increasing the field strength, however, the non-equilibrium term $\mathcal{C}$ rapidly increases until it overtakes the diffusive one, generating the regime of negative differential mobility. In such regime $\mathcal{D}$ can be much higher than in equilibrium and yet diffusion becomes secondary, as the nontrivial trapping of rings - quantified by $\mathcal{C}$ - becomes the dominant phenomenon. 


\subsubsection{Topology can Sense Disorder}

In this Section I investigated the role played by topology in the motion of un-knotted ring polymers through a gel modelled as a disordered environment. It is remarkable that by comparing the behaviour of linear and circular polymers one can infer the degree of microscopic disorder in the medium (Fig. 6.2). On one hand the findings presented here provide us with an explanation for irregular migration speeds detected in experiments comparing linearised and circular plasmids Mickel et al., 1977, Levene and Zimm, 1987, Trigueros et al., 2001; on the other, they also strongly encourage the speculation that with a proper choice of a "topological" polymeric probe, there is the possibility to explore the properties of complex disordered environments from a novel perspective. Polymers with specific topologies may in fact be exploited to design novel ways of sensing the changes in the microscopic structure of porous environments in a new and non-invasive way, e.g. by looking at their mobilities.

Furthermore, I showed that the trapping of ring polymers by the environment is an effect as strong as the force drifting them. This is because the rings have to disengage from the dangling ends in order to re-establish their migration. This is equivalent to move against an external bias and overcome a potential barrier whose height is proportional to the external bias itself.

The phrase "getting more from pushing less" has recently been used to describe situations in which a higher current can be obtained by lowering the field strength Zia et al., 2002, Baerts et al., 2013. This phenomenon has been shown to well describe the behaviour of ellipsoidal Janus particles in corrugated channels Ghosh et al., 2014. Here, another important realisation of this phenomenon, which is intimately related to the broadly used gel electrophoresis technique, has been illustrated. This is an instance of "negative response" and can be interpreted with recent advances in the field of non-equilibrium statistical mechanics. The covariance between displacement and average experienced force, which is zero in equilibrium conditions, may become the dominant contribution to non-equilibrium susceptibilities (as the mobility) and overtake the diffusion term to render the response negative.

\subsection{Gel Electrophoresis of DNA Knots}

In the previous Section I studied the behaviour of un-knotted DNA rings and linear strands. In this Section I will instead focus on knotted DNA rings and their behaviour in (2D) gel electrophoresis experiments. This topic presents some outstanding questions which require a satisfactory answer in order for experiments to exploit the separation properties of gels at their best. In particular, the formation of an arc (see Fig. 6.7) in 2D gel electrophoresis experiments of DNA knots is largely mysterious Trigueros et al., 2001, Olavarrieta et al., 2002, Trigueros and Roca, 2007, Cebrián 
et al., 2014 and although this is nowadays a broadly accepted empirical fact, there is no accurate theoretical explanation of this phenomenon from first principles. The aim of this Section is to shed some light into the physics governing this phenomenon.

A system of 10 nicked, i.e. torsionally relaxed, DNA loops of $M=512 \sigma \simeq$ 3.7 kilo-base pairs (kbp) knotted DNA chains migrating through a gel with lattice spacing $l=80 \sigma=200 \mathrm{~nm}$, and subjected to a sequence of fields of different strength and direction, as in two-dimensional gel electrophoresis experiments (see cartoon in Fig. 6.6. (a)) is here simulated via Brownian Dynamics (see Ch. 3). As in the previous section, I model the gel as an imperfect cubic mesh, where some of the bonds have been cut (see Ch. 3 , here $p$ is fixed to $p=0.4$ ) in order to simulate the presence of open strands, or dangling ends. The loops are either un-knotted, or form one of the first few simple knots (with up to 9 crossing in their minimal projection Adams, 1994).

The average crossing numbers (ACN) used in this work have been obtained from Kusner and Sullivan, 1994, where the authors computed the ACN corresponding to Möbius energy minimising knotted configurations. The thermally averaged ACN of the samples used in this work has been computed from equilibrated configurations and has been found to be in a one-to-one correspondence to the values in Kusner and Sullivan, 1994 (see Ch. 3), confirming the linear relationship between the ACN of ideal and thermally equilibrated configurations Katritch et al., 1996b (see Ch. 3).

By considering the beads as cylinders with height and diameter equal to $\sigma$, the beads volume fraction results $\phi=\pi N M \sigma^{3} / 4\left(L^{3}-V_{\text {gel }}\right) \simeq 1.3 \cdot 10^{-4}$, much smaller than the value at which the chains start to overlap $\left(\phi^{*} \simeq 2.5 \cdot 10^{-3}\right)$. In other words, the systems are in the dilute limit and inter-chain interactions occur rarely in the simulations.

As usual, the starting point of the various simulations is a configuration in which simple knotted configurations are placed outside the box. After slowly pulling the rings inside the gel structure, avoiding any impalement, I placed the boundaries of the simulation box so as to match the boundaries of the gel structure and impose periodicity along all the three coordinate directions. The loops were then equilibrated by running a simulation at zero external field of $510^{6} \tau_{B r}$ time steps, which are disregarded before the external force is switched on. The loops were then subjected to an in silico gel electrophoresis process where a weak electric field is first applied $(\lesssim 50 \mathrm{~V} / \mathrm{cm})$ along the vertical $(z)$ direction, followed by a stronger field $(\gtrsim 150 \mathrm{~V} / \mathrm{cm}$ ) along a transversal, say $y$, direction. I will refer to these two fields as "weak" and "stronger", or "moderate", in what follows. The mean and standard error of various properties, as the mean displacement along $\hat{z}$, are calculated by averaging over the rings in the system and over different runs starting from different (equilibrated) initial conditions. 


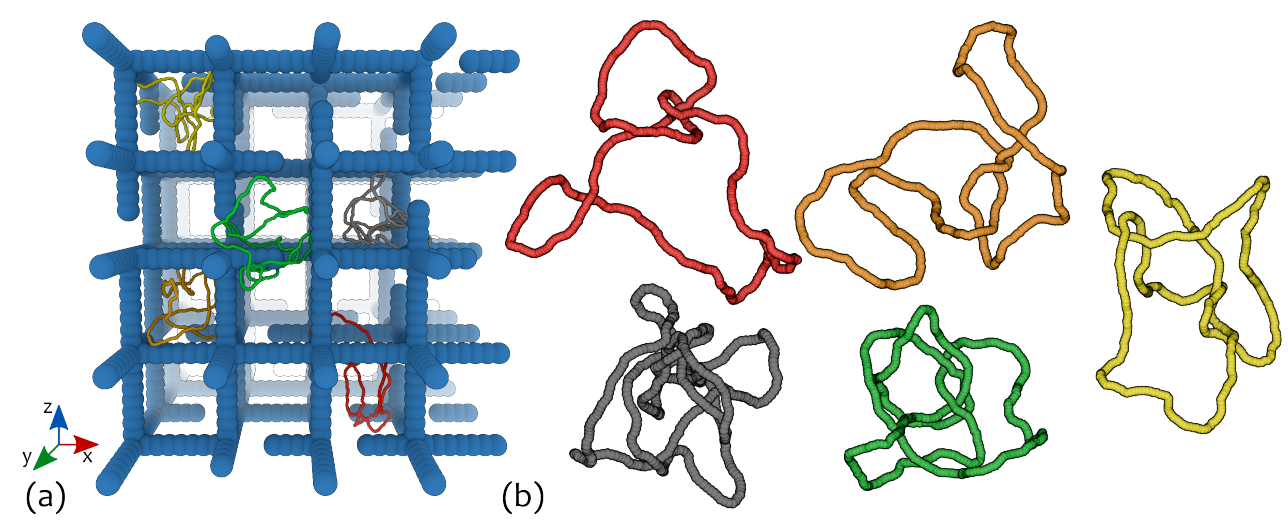

Figure 6.6: (a) Snapshot (to scale) of the model gel with some examples of knotted configurations. Note that to model a physical gel, a simple cubic structure is randomly cut to create dangling ends. (b) Equilibrium configurations of some of the knots considered; it can be readily seen that the size tends to be smaller as the knot becomes more complex. The knots pictured in (a) and (b) are: trefoil $\left(3_{1}\right)$ in red, figure of eight $\left(4_{1}\right)$ in orange, pentafoil $\left(5_{1}\right)$ in yellow, Stevedore's $\left(6_{1}\right)$ in green and "nonafoil" $\left(9_{1}\right)$ in grey.

The external field is modelled as a force $f$ acting on each bead forming the polymers. Assuming that in physiological conditions half of the charges from the phosphate groups are screened by counter-ions Maffeo et al., 2010, Di Stefano et al., 2014 , one can think that each bead $(\sigma=2.5 \mathrm{~nm} \simeq 8 \mathrm{bp})$ contains a total charge of $q_{b}=16 q_{e} / 2$, where $q_{e}$ is the electron charg $\ddagger$, It is therefore possible to map the external force applied onto each bead to an effective electric field $\boldsymbol{E}=-\boldsymbol{f} / q_{b}$. Although this mapping is a crude approximation of the Coulomb interaction between the charged DNA, the ions in solution and the applied electric field, I find that one can recover a "weak field" behaviour of the knotted samples, i.e. linear increase of the speed as a function of their ACN, up to $\sim 50 \mathrm{~V} / \mathrm{cm}$, which is roughly comparable with the field intensity used in experiments. In this section the fields used range from $E=1.25 \mathrm{~V} / \mathrm{cm}$ to $E=625 \mathrm{~V} / \mathrm{cm}$ s

\subsubsection{Non-monotonic Speed of DNA Knots in Gel}

By monitoring the trajectories of the knots through the gel I computed the average speed of their center of mass along each of the field directions (see Appendix E and Fig. 6.7a-b). As expected, the mobility along the direction of the weak field increases with the topological complexity of the configurations. Along the direction of the moderate field, however, the mobility of the knots displays a non-monotonic behaviour. In particular, the un-knot now moves faster than either the trefoil or the $4_{1}$ twist knot, and has an average speed similar to the $5_{1}$ knot. This non monotonic behaviour of the knot mobility, as a function of the ACN, was previously

\footnotetext{
${ }^{\ddagger}$ Differently to the previous Section, the parameters used here are closer to the ones used recently in the literature Di Stefano et al., 2014.

${ }^{\S}$ This range corresponds to a range of forces acting on each bead from $f=1.610^{-4} p N$ to $f=0.08$ $p N$ or total forces $F=M f$ from $0.08 p N$ to $20 p N$, which are compatible with experimental values.
} 

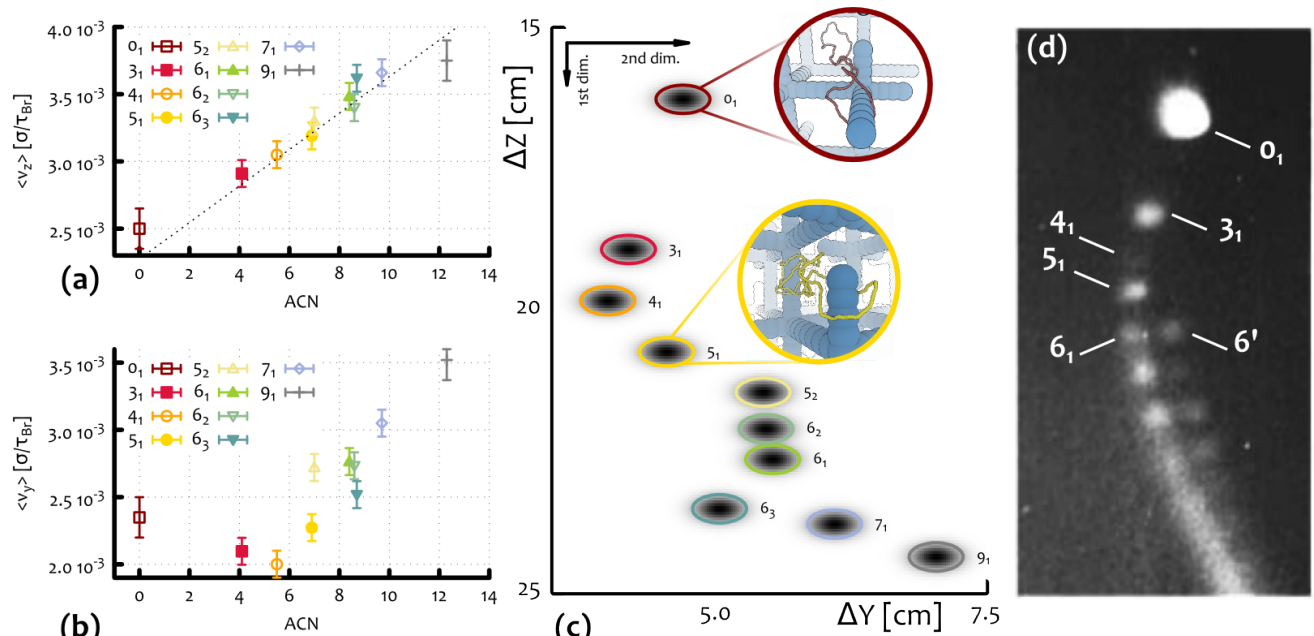

Figure 6.7: In silico 2D gel electrophoresis for knotted DNA loops. (a) Average velocity of different types of knotted polymers along the direction of the weak electric field: $E_{1}=$ $f_{1} / q_{b} \simeq 50 \mathrm{~V} / \mathrm{cm}$. The dotted line indicates the linear increase with the ACN. (b) Average velocity of different types of knotted polymers along the direction of the moderate field $E_{2}=f_{2} / q_{b}=150 \mathrm{~V} / \mathrm{cm}$. (c) $2 \mathrm{D}$ reconstruction of the spatial distribution of the knots as Gaussian probability distributions centred in $\left(v_{z}(\mathcal{K}) t_{z}, v_{y}(\mathcal{K}) t_{y}\right)$ where $v_{z}(\mathcal{K})$ and $v_{y}(\mathcal{K})$ are the average speeds along, respectively, the weak and stronger field direction of knot type $K$, as found by Brownian dynamics simulations. The electrophoretic run-times correspond to $t_{z}=0.810^{10} \tau_{B r} \simeq 5 \mathrm{~min}$ and $t_{y}=2.610^{10} \tau_{B r} \simeq 16 \mathrm{~min}$. The spread of each spot has been estimated by looking at the standard deviation of $v_{z}(\mathcal{K})$ and $v_{y}(\mathcal{K})$. (Examples of single trajectories along the $z$ and $y$ directions are reported in Appendix E Fig. E.1). (d) Outcome of a 2D gel electrophoresis experiment performed on P4 viral DNA (10 kbp) at $0.4 \%$ agarose concentration (from Arsuaga et al., 2005).

observed in typical experiments with torsionally relaxed DNA knots Trigueros et al., 2001, Arsuaga et al., 2002, Arsuaga et al., 2005, Trigueros and Roca, 2007, Cebrián et al., 2014.

To better compare our findings with experiments I report, in Fig. 6.7k, the spatial distribution of the knots as Gaussians centred in $\left(v_{z}(\mathcal{K}) t_{z}, v_{y}(\mathcal{K}) t_{y}\right)$ where $v_{z}(\mathcal{K})$ and $v_{y}(\mathcal{K})$ are the velocities along $z$ (weak field direction) and $y$ (stronger field direction) of knot $\mathcal{K}$, while $t_{y}$ and $t_{z}$ the electrophoretic run-times. The width of the Gaussians is set to be proportional to the standard deviation of the velocities. The resulting spots can be seen as the in silico analogue of the ones observed in gel electrophoretic experiments. Note that the combination of a monotonic behaviour along the weak field direction with a non-monotonic one along the stronger field, gives rise to the arc shape distribution of the spots characteristic of $2 \mathrm{D}$ electrophoresis experiments run either on knotted configurations or on or supercoiled plasmids Cebrián et al., 2014 (see Fig. 6.7d).

It is interesting to ask whether one can observe the electrophoretic arc also in simulations where the gel is a regular cubic mesh, i.e. a mesh with no dangling ends, as this has been so far the typical way to model an agarose gel Weber et al., 2006b, Weber et al., 2006a. Remarkably, unlike the case of gel with dangling ends, 
also called "irregular" hereafter, no example of non-monotonic behaviour of the knot mobility is found for regular gels (for comparison see Fig. 6.7c) and Fig. E.1. This result is in line with previous simulations based on lattice knots in regular gels Weber et al., 2006b) and persists for different field strengths $(1.25-600 \mathrm{~V} / \mathrm{cm})$ and gel pore sizes $(200-500 \mathrm{~nm}$ ) (see Appendix E). Hence, the findings reported here strongly suggest that the causes for the non-monotonic behaviour, observed in the case of irregular gels, are to be sought in the interaction between the knots and the gel dangling ends. It is worth highlighting that in Ref. Weber et al., 2006b, Weber et al., 2006a the authors already investigated the behaviour of knots under weak and strong fields. In their work, they reported a monotonic behaviour between knot complexity and its speed even at very high fields. Although they observed that for electric fields stronger than a certain critical value more complex knots migrated slower than simpler ones, their findings would not lead to the arc pattern observed in experiments of $2 \mathrm{D}$ gel electrophoresis but to a straight line where simpler knots would travel further than more complex ones. In light of this, the results presented here, that the presence of dangling ends in the gel is crucial for retrieving the non-monotonic speed of the knots, while regular gels always lead to monotonic separations, do not contradict previous results (and in particular the ones in Ref. Weber et al., 2006b, Weber et al., 2006a ) but instead suggests a different explanation for the experimentally observed, and yet unexplained, arc pattern.

This conjecture is also supported by the fact that linear (open) DNA samples, are frequently observed to migrate faster than covalently closed (unknotted) ones, in gel electrophoresis experiments performed in both strong and weak fields Trigueros et al., 2001, Arsuaga et al., 2005, Trigueros and Roca, 2007, Cebrián et al., 2014 and it is also in line with the results from Section 6.1, where I showed that the presence of dangling ends slow down polymers with closed topology.

\subsubsection{Entanglement with Dangling Ends}

Having established that the presence of dangling ends in gels severely affect the transport properties of the knotted DNA loops under moderate electric fields, it is natural to look at the possible mechanisms ruling this phenomenon.

The typical trajectories and average extension of some knotted loops, as they move through a regular model gel and a gel with dangling ends are markedly different at moderate fields (see Fig. E.1). In a regular gel, knots respond to the field, by shrinking their size so as to channel through the pores of the gel more efficiently. This mechanism, also known as "channelling", for which polymers squeeze through the gel pores, has already been observed in previous works Mohan and Doyle, 2007b, Mohan and Doyle, 2007a, and it was previously conjectured to play a role in the nonmonotonic separation of DNA knots in gels, as more complex knots could deforming differently when squeezing through the pores [Cebrián et al., 2014]. On the other 
hand, as discussed in the preceding section, I find that this behaviour is not sufficient to explain the electrophoretic arc, as for regular gels I always observe a monotonic separation of the knots as a function of the ACN (see Fig. E.1).

On the contrary, in the case of irregular gels, knotted loops are much more prone to entangle with one (or more) dangling end (see insets of Fig. 6.7 for some examples). These entangled states (or "impalements") require some time to be unravelled and this is the reason of the anomalously long pauses observed in the knot trajectories (see Fig. E.1). Clearly, as the DNA gets longer, "impalements", which can either be parallel or perpendicular with respect the direction of the field, become progressively more likely. As a matter of fact this could be one of the reasons why it is in practice infeasible to perform efficient gel electrophoresis experiments with circular DNA longer than $10 k b p$ Dorfman, 2010]: at these sizes impalements are so frequent that they may cause DNA breakage.

In analogy with the phenomenon of threading, which slows down the dynamics of un-knotted loops either in a melt or in a gel (see Ch. 4 and Appendix B), and that of "crawling" of knots around obstacles Weber et al., 2006a, it is reasonable to expect that more complex knots will take longer to disentangle themselves from an impalement. One can argue that this mechanism, when competing with the reduced Stokes drag of more complex knots in gels, is ultimately responsible for producing a non-monotonic dependence as a function of their complexity, i.e. their ACN.

\section{Competition between Size and Knottedness}

Given that "impalement" events are key factors in determining the mobility of DNA knots within gels with dangling ends, it is important to find a way to define and measure this entanglement. Impalement may occur with dangling ends oriented along several directions (see examples in Fig. 6.7(c)) but it is reasonable to expect that all these events involve a similar mechanism in which, i.e. one dangling end "pierces through" the knot.

To quantify the degree of knot-gel entanglement, I consider an equilibrated knot configuration in the gel, and project it on the plane perpendicular to the field direction. I then choose randomly a base point $P$, at a distance from the projection plane that is much bigger than the radius of gyration of the projected configuration, $R_{g}^{\text {proj }}$. Starting from $P$ it is possible to draw an arc which pierces only once the projection plane at a point, $Q$, chosen randomly, with uniform probability within a disk of radius $R_{g}^{\text {proj }}$ and centred in the center of mass of the projected configuration. The arc and the plane define a semi-space and one can close the path with a second arc connecting $Q$ and $P$ and living in the other semi-space (see Fig. 6.8 a). To assess whether this circular path interacts topologically with the knotted configuration, one can compute the absolute value of the linking number, $|L k|$, between the circular path and the knot. Fig. 6.8 b and Fig. 6.8 show the result of this procedure when 
(a)
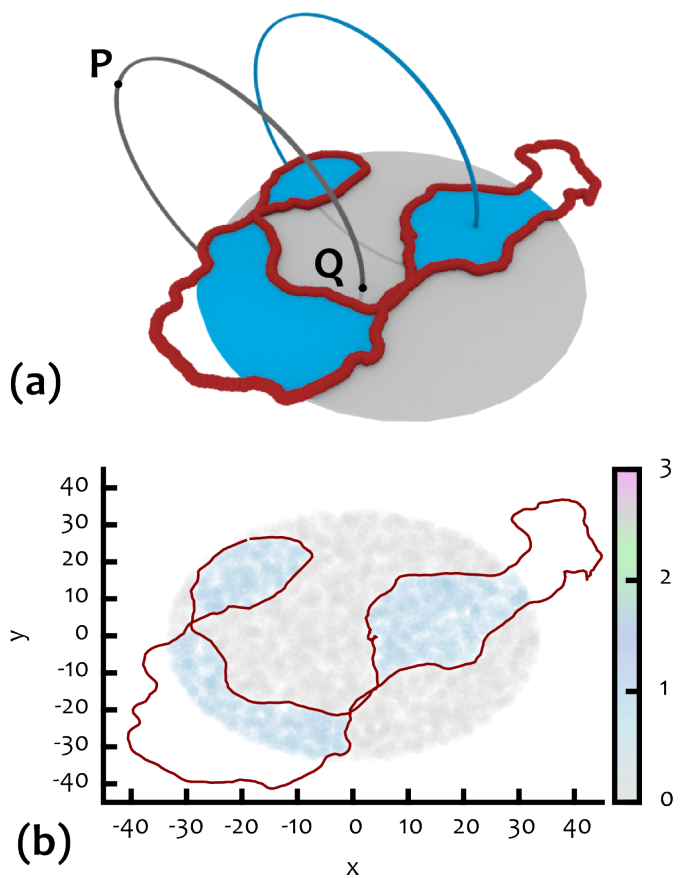

(c)
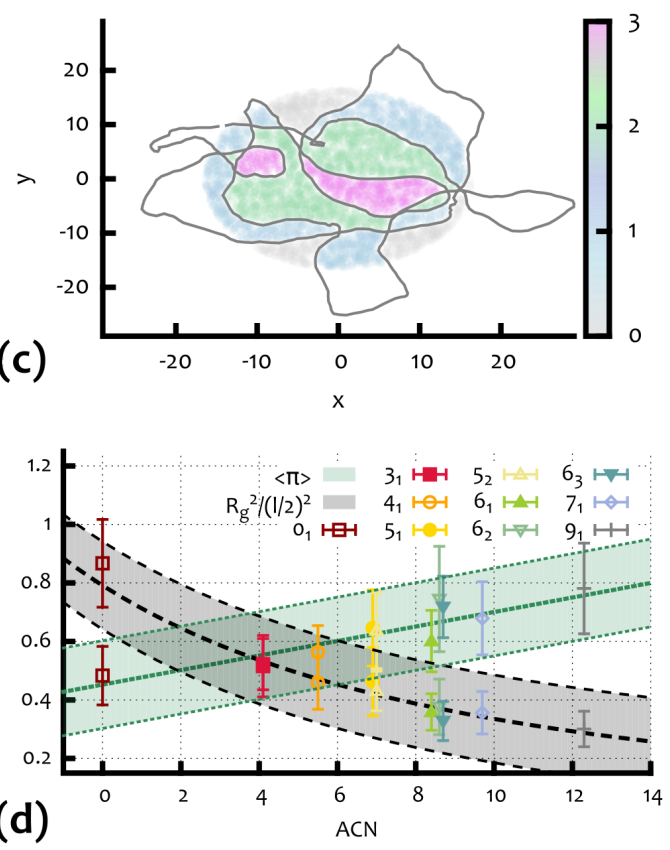

Figure 6.8: (a) Sketch of the procedure used to define the piercing, or entanglement number: for a given projection of the configuration, the crossings define a set of regions whose intersections with the disk of radius $R_{g}^{\text {proj }}$ are highlighted in blue. Starting from different points $P$ far away from the projections closed paths ( 2 in the example) that pierce once the disk at different locations $Q$ are built at random. The absolute value of the linking number is then computed between the knot configuration and each closed path. The average over the set of closed paths is finally taken: this is the average entanglement number. (b) and (c) show the results of the procedure described in panel (a) for a configuration with knot type $9_{1}$ (c) and for an un-knotted configuration (b). The regions are coloured according to the computed absolute value of the linking number (see colour map at the right). Note that for the $9_{1}$ case there are regions of high (3) $|L k|$, which are more prone to become entangled with the dangling ends of the gel. (d) shows the average entanglement number $\langle\pi\rangle$ and the mean squared radius of gyration divided by $(l / 2)^{2}(l$ is the gel pore size) for different knot types classified in terms of $\mathrm{ACN}$.

applied to two different knotted loops. By averaging $|L k|$ over several closed paths with different $Q$, and over different knot configurations, one can define the "average entanglement number", $\langle\pi\rangle(\mathrm{AEN})$ as the measure of the degree of entanglement between the knotted loops and the surrounding gel.

In some sense, this procedure is reminiscent of the one used to assess the "group" of a knot Adams, 1994, or one might think of this as "piercing" the Seifert surface of the knot and taking the average over many "piercings" $(\langle\pi\rangle)$ (see Ch. 3 and Appendix A.

From Fig. 6.8(d) one can notice that $\langle\pi\rangle$ grows approximately linearly with ACN; as one would expect, more complex knots can, on average, become more entangled with the surrounding irregular gel. It is interesting to compare this behaviour with that of the mean squared radius of gyration normalised with respect to half the gel pore size (see Fig. 6.8 d): unlike the average entanglement number, the average 

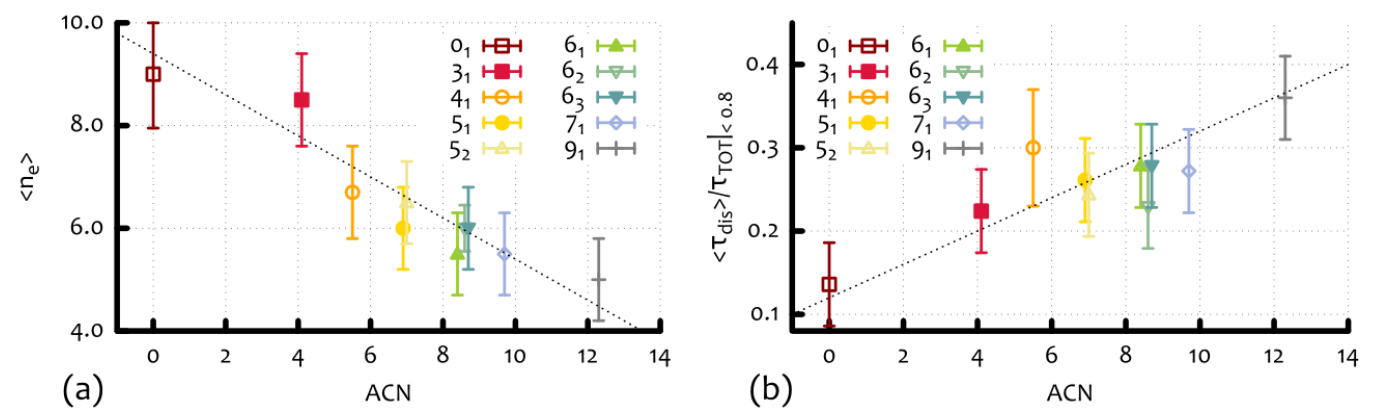

Figure 6.9: (a) Average number of events in which the knot is entangled with the surrounding gel (entanglement events) as a function of ACN. (b) Average disentanglement time as a function of ACN. In these estimates only entanglement events with duration shorter than $200 \tau_{B r}$ are considered, in order to remove the "head on" impalements.

extension of the loop is, to a good approximation, inversely proportional to the ACN, i.e. to the knot complexity. This corresponds to the well-known fact that, for a given loop contour length, more complex knots are on average less extended Stasiak et al., 1996, Piili et al., 2013 (see also the equilibrium configurations in Fig. 6.6).

The plots in Fig. 6.8(d) suggest a possible interpretation of the non-monotonic mobility of the knots in irregular gels based on the interplay between the average size and the degree of entanglement with the gel. On one hand more complex knots, being smaller in size, experience less frequent collision with the gel and hence should travel more easily through it: this is just another variant of the Stokes friction argument discussed previously. On the other hand, once knot-gel collisions occur, more complex knots experience a more intricate entanglement with the gel (higher values of AEN are more probable) that will take longer to be unravelled Stasiak et al., 1996, Weber et al., 2013, Weber et al., 2006b, Weber et al., 2006a.

\section{Hitting and Waiting Times}

The above argument suggests the existence of two time scales in the process: one is the time $\tau_{f}$ between two successive knot-gel collisions yielding a local entanglement; the other, $\tau_{\text {dis }}$, is the time needed by the knotted loop to fully disentangle from the impalement. The time scale $\tau_{f}$ increases as the knot average size decreases and hence increases with knot complexity $(\mathrm{ACN})$. In other words more complex knots experience, on average, less collisions with the gel than their simpler counterparts. The second time-scale, $\tau_{\text {dis }}$, is instead an increasing function of $\langle\pi\rangle$ (see Fig. 6.8(d)) and hence of the knot complexity (measured in terms of ACN). According to this picture, the slowest topoisomer in an irregular gel with a given lattice spacing will be the one with the "best" compromise between a high rate of collisions, and a sufficiently high value $\langle\pi\rangle$.

To investigate more quantitatively the dependence of $\tau_{f}$ and $\tau_{\text {dis }}$ on the knot type $(\mathrm{ACN})$, one can analyse the trajectories of the knotted loops in the gel by 
computing: (i) the average number of times a knot arrests its motion in the gel (entanglement event), $\left\langle n_{e}\right\rangle$, and (ii) the distribution of the duration of these entanglement events. As specified in Appendix E, the duration of the entanglement events can be identified as the time intervals where the spatial position of the centre of mass of the configuration deviates significantly from the expected collision-free field-driven linear motion with speed $v_{\text {free }}=F_{z, y} / M \zeta=f_{z, y} / \zeta$.

As reported in Fig. E.2, the average fraction of time in which the knot is trapped, $\tau_{w} / \tau_{\text {tot }}$ ( $\tau_{\text {tot }}$ is the time of the full trajectory), is a non-monotonic function of the ACN, in line with the result on the mobility under moderate field (Fig. 6.7(b)). In Fig. 6.9(a) I also show that the average number of entanglement events $\left\langle n_{e}\right\rangle$ decreases with the knot complexity (i.e. ACN). On the other hand, the distribution of the duration of these events displays an intriguing bimodal shape, with two peaks occurring respectively at short and very long times (see Fig. E.2. The peak at long times can be interpreted as the signature of head-on collisions with the gel, where the dangling ends involved are opposite to the direction of the knot motion. The resulting entanglement is, in this case, very difficult to unravel especially in presence of a strong electric field (see for instance the inset of Fig. 6.7 for the unknot). Nonetheless, either if this long-time peak is excluded or not from the statistics, the characteristic disentanglement time $\tau_{\text {dis }}$ turns out to increase (linearly) with the knot complexity, i.e. with $A C N$ (see Fig. 6.9(b)).

One can assume that this bi-modal shape is due to a shift in the energy barrier that the knots have to overcome in order to disentangle from the dangling ends. In particular, one can think to this process as an Arrhenius process, where the energy barrier is a function of the length (projected along the field direction) of the dangling end, the knot complexity and, more importantly, the magnitude of the external field. When this is too strong, disentanglement events are very rare, and all knots will end up being permanently entangled with the gel structure; on the other hand, when this is too weak, the typical disentanglement time is very short, and the dependence of $\tau_{f}$ as a function of the ACN dominates the motion of the polymers, re-establishing the usual linear relationship.

\subsubsection{An Equivalent Random Walk Description}

As shown in previous sections, 2D electrophoresis experiments and Brownian Dynamics simulations of knotted loops in irregular gels are in qualitative agreement under many aspects.

In this section I propose a simple model that reproduces the main findings of the simulations and furnishes a simple but accurate way to predict the arc shapes of the experimental patterns as a function both of the knot complexity and of the loop contour length. In this model, I describe the knotted loop moving within the irregular gel as a biased random walk on a $1 \mathrm{D}$ lattice, i.e. a random walk that moves 
to the right (direction of the external force) unless it is trapped into an entangled state (due to impalement) with probability $\lambda_{e}(\mathcal{K})=\tau_{f}^{-1}(\mathcal{K})$ (see Appendix $\mathrm{E}$ for more details). Once in the entangled state, the walker has to wait a given amount of time that is picked randomly from a bimodal distribution consisting of an exponential decay, modelling the short time disentanglement, and a smaller probability peak at large times, describing the long disentanglement time from a head-on collision.

In this simple description the only relevant parameters are the hitting rate and the parameters characterising the bimodal distribution of waiting (i.e. disentanglement) times. Once the values of these parameters are set to reproduce the data reported in Fig. 6.9, the model can be used to predict the mobility of the electrophoretic arc as a function of ACN. As shown in Fig. 6.10 this procedure reproduces, with remarkably good agreement, the simulation data and, in particular, it captures the physical mechanism leading to the non-monotonic mobility at moderate field. Note that, as the random walker solely moves to the right, the field strength does not enter explicitly into the model, but rather, it is considered to be neither too weak to allow backward moves or too strong to completely suppress disentanglement of the knots. Both these cases in fact would not deliver an electrophoretic arc.

More importantly, once the parameter values of the biased random walk model are set for a given pore size of the gel, $l_{1}$, their values for a different pore size, $l_{2}$, can be estimated from general arguments. One can therefore use this simplified model to predict the moderate field mobility and the shape of the electrophoretic arcs of DNA knots in gels of variable pore size, e.g. tuned via agarose concentration Pernodet et al., 1997 or nano-wire growth cycle Rahong et al., 2014. The plots presented in Fig. 6.10(b) suggest that tighter gels give rise to more curved (or deeper) arcs where the slowest knot has a higher ACN with respect to sparser gels. Moreover, since the entanglement rate $\lambda_{e}$ and the disentanglement time $\tau_{\text {dis }}$ (both of these relative to the same quantities for the un-knot) should depend only on the ratio between the knot extension and the gel pore size $l$, a similar trend should be observed also by increasing the DNA loop contour length by keeping fixed $l$. This is in qualitative agreement with experiments, as electrophoretic arcs are straighter for shorter DNA molecules (Fig. 6.10(d)). A further quantitative prediction can be drawn from these findings is that the relative position of the three 6 -crossing knots can be controlled by tuning the pore size (Fig. 6.10) of the gel. Indeed the size of the pores determines whether the $6_{1}$ Stevedore's knot is to the left or to the right of the minimum of the mobility curve: in the former case $6_{1}$ will moves faster in the gel than the $6_{2}$ and 63 knots (which, having higher ACN have also higher AEN), while in the latter case it will move more slowly. This detailed prediction could be tested in future electrophoresis experiments with knotted DNA loops moving within different gels. 


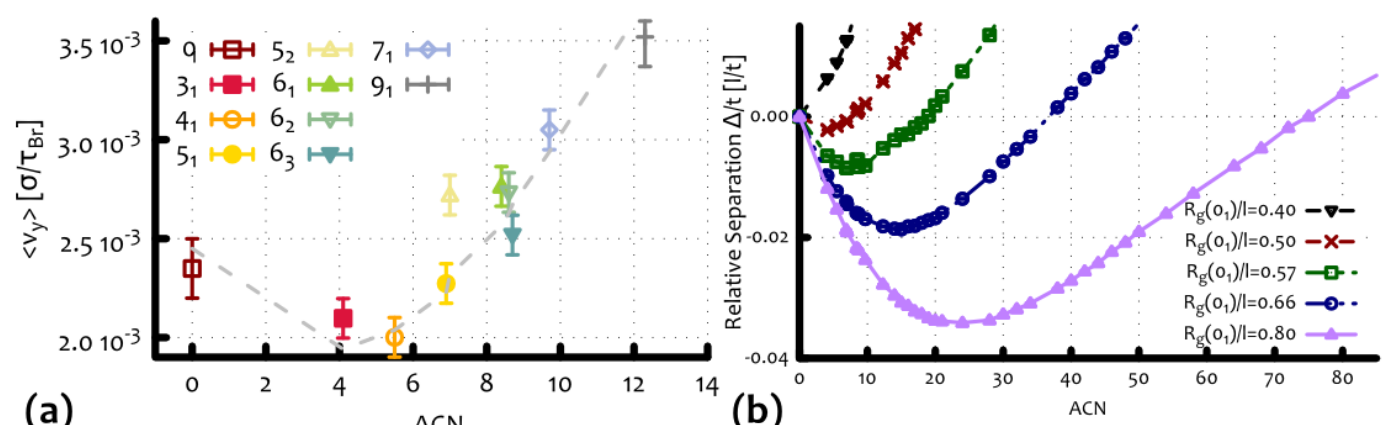

(a)
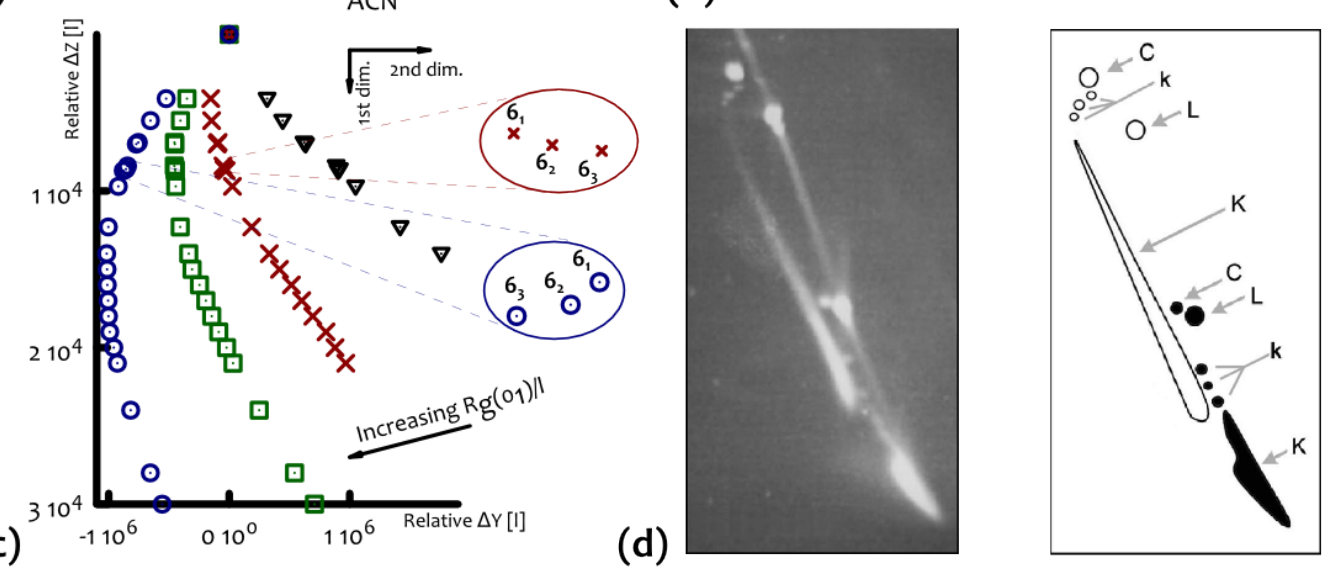

Figure 6.10: (a) Average speed along the direction of the moderate field from Fig. 6.7(b). The dashed line is obtained from the biased continuous random walk model, and corresponds to the (shifted and rescaled) red curve in (b). (b) Average relative separation (in units of lattice spacing over time) of the knots as a function of the ACN for different parameters, as predicted by the continuous random walk model. The grey dashed line in (a) is obtained by shifting the red curve in (b) by the value of $\left\langle v_{y}\right\rangle\left(0_{1}\right)$ and rescaling it by the free velocity $v_{\text {free }}$. (c) Reconstruction of a 2D gel electrophoresis experiment from the data in (b) and zoom over the relative position of the family of 6-crossings knots for two cases in which the minimum of the arc is at their left and their right. (d) Outcomes of a 2D gel electrophoresis experiments performed on P4 viral DNA with different lengths, respectively 4.7 (black) and 10 (white) $k b p$ at equal agarose concentration $(0.4 \%$ ) (reproduced from Trigueros and Roca, 2007 ).

\subsection{Conclusions}

In this Chapter I have studied the behaviour of linear, un-knotted circular and knotted polymers drifting in a disordered gel. There are several open questions in this field, which are all pertinent to the theoretical understanding of gel electrophoresis techniques, such as the anomalous mobility of ring polymers in gels, and the nonmonotonic speed of knotted DNA samples.

In Sec. 6.1 I showed that the mobility of ring polymers moving through a physical gel is strongly affected by their interaction with the surrounding environment. Ring polymers can be slowed down by several order of magnitude by becoming entangled with the gel structure. This is strongly correlated with the fact that the topology of the rings is closed, and has to remain closed at all times. Linear polymers can 
disentangle from the gel structure with virtually no slowing down (or at least much more quickly than rings).

Because the entanglement of ring polymers becomes stronger as the field driving the polymers is increased, there exists a critical field $E^{*}$ above which ring polymers will travel slower than their linear counterparts. In the limit of very large external fields, ring polymers show very low gel mobilities, as experimentally observed Mickel et al., 1977, Cole and Åkerman, 2003, Stellwagen and Stellwagen, 2009.

It is also worth pointing out that this peculiar behaviour of rings in gels can be used to probe the microscopic disorder of porous materials. Ring polymers could in fact be adopted as "topological probes" and their mobility compared with that of linear samples, thereby providing us with information regarding the microscopic disorder of materials.

In Sec. 6.2 I studied a system of knotted polymers moving through a gel possessing rigid dangling ends. At weak fields one can recover the well-known linear relationship between migrating speed and knot-type while at stronger fields, one can instead observe a non-monotonic behaviour. This puzzling feature, has been tackled in the literature but never fully explained. In particular, in Ref. Weber et al., 2006b, Weber et al., 2006a the authors studied the effect of crawling of knots around gel obstacles and dragged by weak and strong fields. Although they reported a very interesting field inversion effect (where more complex knots were observed to travel slower than less complex ones) their findings would nonetheless lead to a monotonic response of the knots' speed as a function of their complexity, i.e. the knots would from a straight line in a $2 \mathrm{D}$ gel electrophoresis experiment. On the other hand, the findings presented in this Chapter show that in order to obtain a non-monotonic response of the knots speed, the presence of dangling ends is key. Only by accounting for dangling ends, the non-monotonic speed, and therefore, the re-entrant envelope displayed by the knotted DNA strands, can be retrieved. This pattern can, in fact, be better understood by taking into account the topological interactions, or impalements, of the knots with the irregularities of the surrounding gel. While more complex knots assume more compact configurations, and hence smaller Stokes friction than simpler knots, they also experience more complex impalements with the gel and hence longer disentanglement times. These two competing effects give rise to the non-monotonic speed of the knots observed in the experiments, a feature that, remarkably, is absent for knotted loops moving in a regular gel (i.e. no dangling ends). It is also remarkable that the patterns observed in the experiments can be reproduced via a simple Biased Random Walk model, with "topology-dependent" hitting and disentangling times, where the depth of the arc pattern can be finely tuned via few key parameters. Even though it is clear that the findings reported in this Chapter can reproduce the arc pattern thanks to the addition of dangling strands in the gel, it is still unclear what is the microscopic disentanglement mecha- 
nism undergone by the knots once they interact with the gel. While the results show that more complex knots take longer time to disentangle, the reason why this is the case is still an open question. This might be due to complex tightening around the dangling ends, which would be knot dependent, or due to some correlations between the strands that need to overcome the obstacle in order for the knot to re-establish its motion. Yet unexplained, this complex disentanglement mechanics is surely worth exploring in the future.

It is my hope that the results presented here will inform and stimulate further experimental and numerical investigations of the role of topology in the anomalous electrophoretic mobility of circular and knotted polymers. I aimed to provide models as simple as possible while retaining the essential physics, and to provide sound and testable outcomes that can inform more accurate experimental set-ups to separate bio-polymers of different topology.

It is also worth stressing that this Chapter is a clear example of how topology not only affects the intrinsic properties of a polymer, such as its size (gyration radius), but also how it interacts with the surrounding environment. The "topological interaction" of a knotted DNA with the irregular environment of a physical gel, for instance, has been here shown to play a key role in driving the non-monotonic electrophoretic mobility of DNA topoisomers. 

light of the results of this Thesis, one could argue that joining the ends of a linear polymer has had an astronomical effect on the polymer static, and dynamic, behaviour and it has had a much deeper consequences than one might have naïvely anticipated.

From the change of universality class (Ch. 2 to the possibility of displaying a kinetically arrested state at any temperature (Ch. 4) and to have different interactions with the surrounding environment depending on their knottedness (Ch. 6), ring polymers have been shown to offer a richness of behaviours whose full understanding is possibly one of the main theoretical challenges for the Polymer Physics community in the years to come.

All of this can be, rather simply, captured by the fact that ring polymers assume well defined topological states, i.e. (un-)knotted and/or (un-)linked, and can interact via three types of inter- and intra-chain topological interactions: threading, linking and knotting. Clearly, these cannot be even defined in systems of linear, or topologically trivial, chains.

These interactions are here investigated in Ch. 4, 5 and 6, and in each of them I have tried to focus on specific biologically-oriented examples and applications which allowed me to better grasp the meaning and consequences of these topological interactions in real-world problems.

In Ch. 4 I have shown that threadings play a major role in the dynamics of dense solutions of rings. In particular, under some conditions which are not too far (and possibly not far at all!) from experimentally realisable set-ups, the motion of dense solution of rings can be dominated by correlations induced by topological interactions (or "topological correlations") which lead a kinetic arrest, or a "topological glassy" state.

Linking between rings has been explored in Ch. 5. and in particular in the case 
of the Kinetoplast DNA. The remarkable and unique structure formed by the mitochondrial genome of organisms of the class Kinetoplastida offer an exceptional example of how important this topological interaction is even in their bare existence and survival. I showed that the topological regulation of the network of linked rings thereby formed can be understood in terms of simple bio-physical models which, despite their simplicity, can provide us with some fresh insight into this mysterious structure.

Last, but not least, knotting represents one of the most studied and historically important topological (self-)interactions. Despite the enormous effort in characterising the behaviour of knots in various contexts, much is still to be understood. In Ch. 6 I brought my (little) contribution toward the general understanding of this topic by investigating the puzzling and unexplained non-monotonic behaviour of knotted DNA samples in gel electrophoresis experiments. This long debated phenomenon has been found to be intimately related to topology as both, the knots' size and their "topological interactions" with the environment, are controlled by their knottedness.

Above all, I have here tried to take inspiration from the biological world to ask, and answer, questions which could be relevant and interesting for a broad spectrum of researchers. The scope of the work has always been to provide an accurate, although coarse-grained, description of the systems and to retain the key elements in play. The objective that I set myself was to provide clues toward a deeper understanding of the subjects and I, in particular, aimed to generate experimentally testable predictions and to inform further experiments which are, after all, the final proof of any theory. 
Appendices 


\section{A \\ Identifying Knots}

Identifying unambiguously any knot type is, currently, impossible. The design of an algorithm that will make this possible is one of the biggest challenges for knot theorists in the years to come. Mathematically, knot types are usually identified by analysing the topological properties of the knot complement, i.e. the manifold formed by removing the knot from the space in which it is embedded $\left(S^{3}-\mathcal{K}\right)$, or the topological properties of the Seifert surface $\mathcal{S}$ constructed from a knot diagram representation of a knot Adams, 1994]. In practice, these procedures are hardly translated into automated algorithms that can be repeated over thousands of knots. For this reason it is often preferred to use simpler methods, although less reliable. One of these methods is the computation of polynomials, such as the Alexander polynomial.

The construction of the Alexander polynomial starts from a knot diagram a knot (see Fig. A.1(a)). Being a knot diagram a 2D representation of a 3D object, it is not unique but depends on the perspective chosen for the projection. On the other hand, it can be shown that two knot diagrams of the same knot are equivalent, i.e. can be transformed into one another via a sequence of moves called "Reidemeister moves" (see Fig. A.1). In addition, knot diagrams that cannot be transformed into one another belong to different knot types. There are several quantities that can be calculated from knot diagrams, e.g. the minimal crossing number, the Dowker code, the bridge number or the Alexander and Jones polynomials. Here, I will describe the computation of the Alexander polynomial $\Delta(t)$.

The procedure is the following: (1) Assign a direction to the contour and mark the $n$ crossings and $n$ arcs between crossings (see Fig. A.1.(a)); (2) Determine the sign of the crossings using the standard right-hand rule (see Fig. A.1(b)) (3) Construct an $n \times n$ matrix $M$, where the entries of the $x$-th row are

$$
M(x, i)=1-t ; M(x, j)=-1 ; M(x, i)=t ;
$$

if the $x$-th crossing is positive, or

$$
M(x, i)=1-t ; M(x, j)=t ; M(x, i)=-1 ;
$$




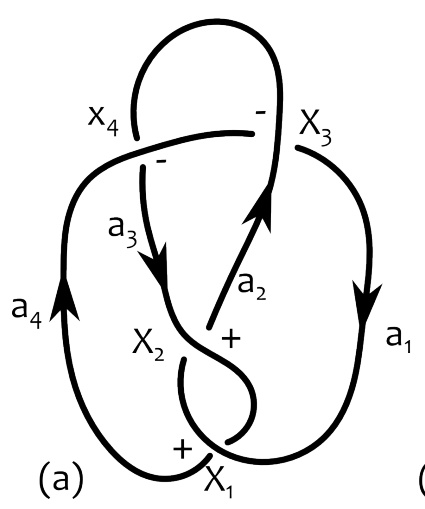

(b)

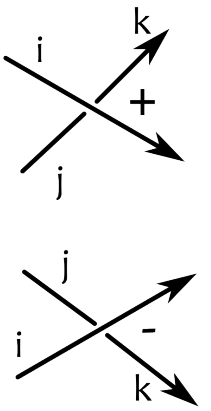

(c)

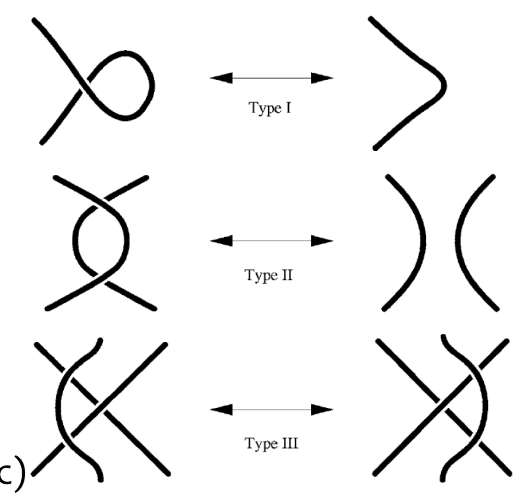

Figure A.1: (a) Knot diagram of a $4_{1}$ knot. (b) Positive and negative crossings. (c) The three types of Redemeister moves, from Orlandini and Whittington, 2007.

if the $x$-th crossing is negative, and where $i, j, k$ are the strands forming the crossing such that $i$ passes over $j$ and $k$. (4) Delete one row and one column, i.e. take one minor of the matrix, and (5) compute its determinant $\Delta(t)$.

From the knot represented in Fig. A.1 one gets:

$$
M(t)=\left(\begin{array}{cccc}
1-t & 0 & -1 & t \\
-1 & t & 1-t & 0 \\
-1 & 1-t & 0 & t \\
0 & t & -1 & 1-t
\end{array}\right) \rightarrow M^{\prime}(t)=\left(\begin{array}{ccc}
t & 1-t & 0 \\
1-t & 0 & t \\
t & -1 & 1-t
\end{array}\right)
$$

whose determinant is $\Delta(t)=-t^{2}+3 t-1$ : the Alexander polynomial of a figure of eight $\left(4_{1}\right)$ knot.

Because Alexander polynomials of different knot diagrams of the same knot can differ up to $\pm t^{m}$ with $m \in \mathbb{Z}$, it is common practice to compute $\Delta(-1)$ (and identify $\Delta(-1)$ with $-\Delta(-1))$ so to avoid ambiguities. The Alexander polynomial is a good knot invariant for a number of practical applications, in particular when dealing with many randomly generated configurations. On the other hand, it cannot distinguish between, for instance, the $8_{20}$ knot and the Granny $3_{1} \# 3_{1}$ knot or between a knot and its mirror image and it is, therefore, to be used with caution. 


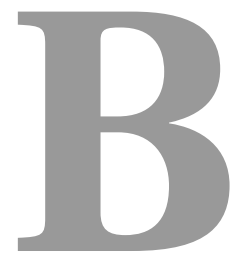

\section{Self-Threading of Rings in Dilute Solutions}

The configuration of sufficiently long unknotted self-avoiding ring polymer unlinked from the gel is a double-folded self-similar branched tree (see Ch. 2), also called a lattice animal Lubensky and Isaacson, 1979, Soteros and Whittington, 1988, Obukhov and Rubinstein, 1994, Whittington and Soteros, 1990]. Because of the fact that lattice animals critical exponent $\nu=1 / 2$ in 3D [Parisi and Sourlas, 1981], the selfdensity $\rho_{s} \equiv M /\left\langle R_{g}^{2}\right\rangle^{3 / 2} \simeq M^{-1 / 2}$ of a large ring polymer in gel is much higher than that of either rings in good solvent, or linear polymers, whose self-density scales as $\rho_{s} \simeq M^{-4 / 5}$. The contact probability between different segments belonging to the same chain is therefore higher. This implies that ring polymers in gel are more likely to hinder their own motion by interacting with themselves, as previously speculated Klein, 1986, Obukhov and Rubinstein, 1994.

In the following, I will be interested in configurations of a ring in gel in which a double-folded segment opens up and is threaded by another double-folded segment of the same chain ("self-threading") (see Fig. B.1). In particular, when a ring polymer is forced to move inside a gel, self-threading can hinder polymer diffusion. Imagine a ring that winds around a strand of the gel and passes through itself, as in Fig. B.1 (a). In this case the threading segment (green) behaves as a temporary "pin" for the threaded one (red), because of the uncrossability constraint. In order for the latter to freely diffuse, the former has to be removed. In the limit of large rings, one can think of a growing number of penetrations which can assume a hierarchical structure (imagine a segment of the polymer that threads through another that then is itself threaded, et cetera). These would have to be undone in order to re-establish free diffusion, consequently increasing the polymer relaxation time. Self-threading is also a candidate for describing the low electrophoretic mobility (at low fields) and irreversible trapping (at high fields) of long ring molecules Viovy et al., 1992, Akerman, 1998, Viovy, 2000. Imagine applying an electric field to the configuration in Fig. B.1(a), in the direction parallel to the green segment. The ring can end up "tightening" itself around the obstacle, and become irreversibly self-trapped (see Fig. B.1(b)), in a process that resembles the tightening of a knot Pierański et al., 2001.

In this Section, I will study how threadings of the chains through themselves can 

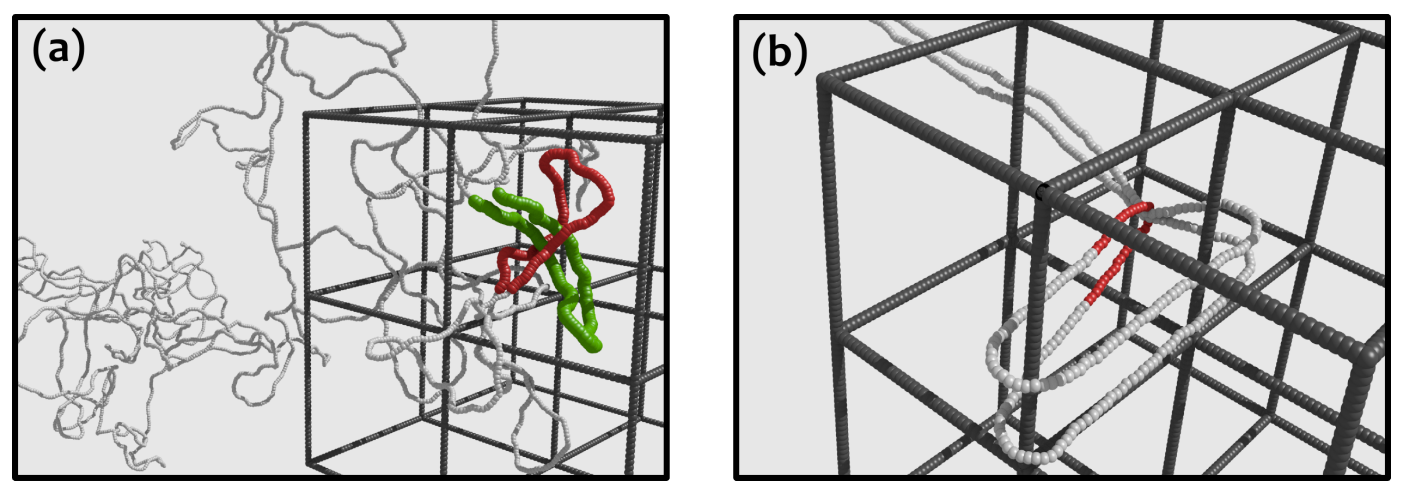

Figure B.1: (a) and (b) show two snapshots of Molecular Dynamics simulation in which a self-threading and a self-trapping events, respectively, are occurring. The colors highlight different segments of the chain. (a) An ending segment of a branch (green) threads through another ending segment of another branch (red). This case is analogous to case B.2(e). Fig. (b) is obtained after a strong electric field is applied to the self-threading configuration (a). The green segment elongates, while the free ends slide backward, until they coincide with the red segment. The configuration thereby generated is "trapped". The gel structure in (a) and (b) is sketched only partially and thinned for clarity.

affect their own motion in dilute conditions. I will investigate how ring polymers behave when diffusing in a 3-dimensional gel by coarse graining the double-folded configurations to a network of beads located in the cells of the gel (see Fig. B.2). This coarse graining procedure maps the problem of simulating ring polymers diffusing in a gel to that of annealed branched polymers diffusing on a lattice, or lattice animals Lubensky and Isaacson, 1979, under a specific set of rules which preserve the topology of the rings. The dynamics is simulated using an equivalent model to the kink-gas diffusion introduced by de Gennes de Gennes, 1971, Cates and Deutsch, 1986 Obukhov and Rubinstein, 1994, suitably modified to correctly take into account the slowing down due to the chains self-threadings. I will show that in the limit of large rings, self-threadings increase extensively with the rings length and the dynamics is consequently dramatically slowed down.

\section{Algorithm and Computational Details}

The coarse-grained model is implemented by means of a Lattice Kinetic Monte-Carlo simulation of isolated lattice animals formed by $M$ beads diffusing on a lattice. In order to simplify the model I will make some assumptions: Firstly, by replacing a double-folded segment of the polymers with a single bead filling a unit cell, I am implicitly assuming that the lattice spacing $l$ of the gel is comparable to the rings' Kuhn length $l_{K}$. The polymers are, therefore, flexible on the length scale of the gel pores. By making this choice, and considering the Kuhn length of dsDNA, i.e. $l_{K}=100 \mathrm{~nm}, \mathrm{I}$ am assuming that the gel pore size is smaller than a typical $6 \%$ agarose gel by a factor of 2 (see Ref. Pernodet et al., 1997]) and, in addition, I simplify further the model by assuming that the gel fibers are rigid (see Sec. 3.2 for further details and evidence in support of these assumptions).

I will focus on systems made of $N=10$ non-interacting ring polymers of length $M=32,64,128,256,512$ beads. The rings do not interact with each-other and therefore behave as if they were isolated. The observables are averaged over at least 3 independent realisations per system, i.e. over $3 N$ rings. The rings are initially 
prepared unlinked from the gel, and therefore they must assume a double-folded configuration: Every unit cell of the gel has both an out-going and in-going polymeric strand (see Fig. B.2). The coarse-graining procedure maps both the out-going and in-going strands to one bead, which has the size of a Kuhn segment, and that spans the entire unit cell. In this way a ring with $2 M$ segments is modelled via a collection of $M$ beads. The rings are treated as lattice animals diffusing on a cubic lattice which is shifted by $\left(\frac{l}{2}, \frac{l}{2}, \frac{l}{2}\right)$ with respect to the gel lattice Weber et al., 2006b. In other words, I model the rings by tracking the backbone and the branches of the lattice animal shapes they assume (see Fig. B.2). The algorithm penalises the creation of new branches and the bending of the terminals by introducing two energies, $E_{\text {branch }}=2 E_{b}(1+\cos \theta)$ and $E_{\text {bend }}=E_{b}(1+\cos \theta)$ respectively, where $\theta$ is the angle formed by consecutive pairs of beads and $E_{b}=k_{b} T$. The motivation for this is twofold: (1) pure translation does not involve any change in energy (Fig. B.2(a)) and (2) branching involves a creation of a new double folded terminal segment which, in terms of angles, contains two $90^{\circ}$ angles with the neighbouring beads $\left(m_{n}\right.$ in Fig. B.2 and one (newly formed) $180^{\circ}$ angle (being a terminal segment). Therefore, the energy penalty for branching is taken as twice the energy required for bending an existing terminal segment (see Fig B.2(b) and (c)).

The details of the algorithm are the following: At every Monte-Carlo time-step, $M N$ beads are randomly selected to attempt a move, so that every bead in the system is considered once, on average, per time-step. When a bead $(m)$ is selected, a move to occupy a neighbouring site $\left(m^{\prime}\right)$ is attempted:

- If $m^{\prime}$ is free, then the move is tested by means of the Metropolis algorithm, where the probability to be accepted is given by

$$
p_{m \rightarrow m^{\prime}}^{\text {branch } / \text { bend }}=\exp \left\{-E_{\text {branch } / \text { bend }}(\theta) / k_{b} T\right\}
$$

- If the site $m^{\prime}$ is occupied by a non-neighbouring bead of $m$ then the move is accepted with probability:

$$
p_{m \rightarrow m^{\prime}}^{\text {branch/bend }}=p_{\text {th }} \exp \left\{-E_{\text {branch } / \text { bend }}(\theta) / k_{b} T\right\}
$$

where $p_{t h}$ is a free parameter in the model and represents the probability of self-threading.

In order to correctly reproduce the hindering of the motion when the terminal segments are threaded, and hence not free to diffuse and contribute to the motion we adopt the following strategy: Once the move has been energetically accepted, we place a new virtual segment at $m^{\prime}$ (represented as a dotted segment/circle in Fig. B.2) and simulate the contour diffusion of an "anti-kink" (or "hole") that starts from $m$ and can annihilate only with one of the terminal beads of the lattice animal (represented as segments/circles shaded in grey in Fig. B.2) or the newly formed segment $m^{\prime}$. For instance, in Fig. B.2(a), once that $m^{\prime}$ is created, a random walk starts from $m$ and can hit either $m^{\prime}$ (which happens most of the times), or the grey bead at the other end of the chain * In the first case, the chain does not move $\left(\mathrm{m}^{\prime}\right.$ is created and removed). In the second case, the chain steps to the right by one site ( $m^{\prime}$ is created and the grey bead removed).

${ }^{*}$ The same procedure can be performed by solving the diffusion equation on the network made by the lattice animal and by assigning a weight equal to the hitting probability to its ends. This method has been also considered and gives the same results as the method I adopt in the following. 


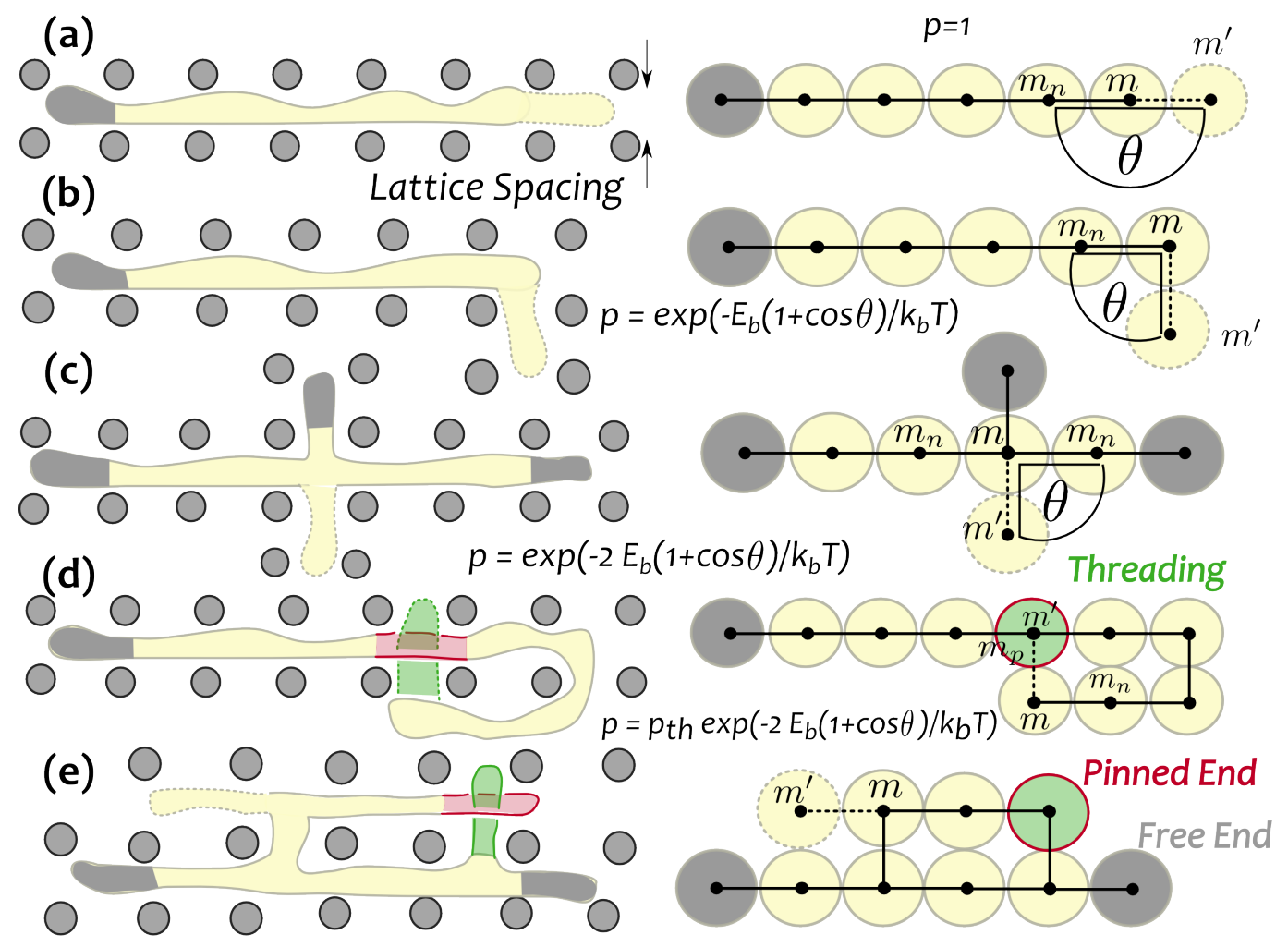

Figure B.2: Cartoon picturing the dynamics of the rings. The rings in the gel (left) are represented by lattice animals moving on the dual of the gel lattice (right). (a) (b) and (c) Represent the moves allowed in a gel: translation, bending and branching, each by retraction of a terminal segment (shaded in grey). See text for more details. (d) With probability $p_{t h} p_{m \rightarrow m^{\prime}}^{\text {branch } / \text { bend }}$ a bead can move onto a site that is already occupied to become an effective pin (green) for the occupant bead $m_{p}$ (red). Computationally, this is implemented by stacking two beads on top of each other on the same site (green bead on top of red one). (e) This is the case in which the threaded segment coincides with an end, in this case such end becomes "pinned". In this configuration, the red segment/bead cannot be retracted, while the green one can contribute to the motion by annihilating with an anti-kink (see text). In this case $m^{\prime}$ can extend only if one of the grey beads or the green one is removed. In the case the red one is attempted to be retracted, the move would be rejected.

Instead of having "kinks" (or segments with stored length) that accumulate along the contour and diffuse until they stop by extending a new segment, we first extend a new segment and then look for a terminal segment which can be retracted in order to accommodate the newly formed protrusion (and conserve the total mass). Since only one kink per time is allowed to travel along the lattice animal, i.e. kinks do not interact, this method is completely equivalent to the kink-gas diffusion introduced by de Gennes de Gennes, 1971, Cates and Deutsch, 1986, Obukhov and Rubinstein, 1994 , with a subtle difference in how this model can take into account long-ranged topological correlations which are key elements in the motion of polymers with a closed topology. In de Gennes' picture, the polymers moved by accumulating mass, or length defects (kinks) along the contour, and by randomly spreading the excess of mass towards the terminal segments, which can extend. Here, we allow for temporary extensions of the terminal segments by creating a pair mass-hole ("kink" - "antikink"). The former immediately settles at the end of the segment $\left(m^{\prime}\right)$, the latter starts a random walk along the chain and stops when either an end, or $m^{\prime}$ itself is 


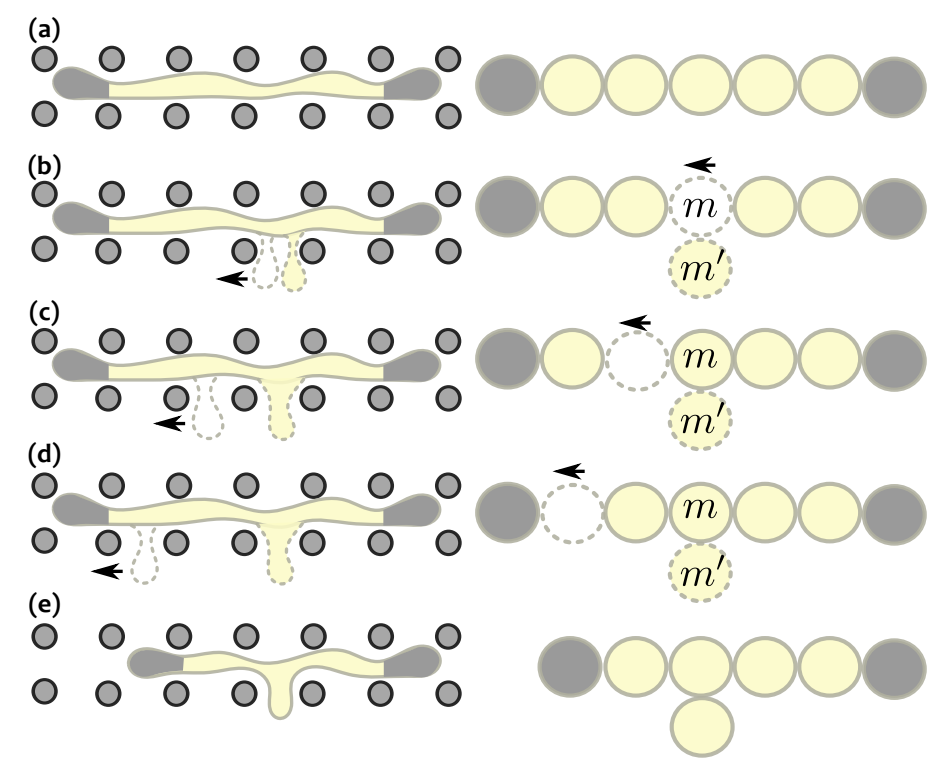

Figure B.3: Sketch of the "kink"- "anti-kink" dynamics. (a) A pair "kink"- "anti-kink" is created at $m$. The kink settles and becomes $m^{\prime}$, while the anti-kink starts a random walk from $m$. (b)-(c) The "anti-kink" travels to the left departing from the kink. (d) The "anti-kink" hits a free end (shaded in grey) and annihilates, generating the configuration in (e). (e) New configuration generated by the algorithm, where the old free end to the left is removed and substituted by a new free end.

hit (see next section for details).

It is also worth noting that it is implicitly assumed that the probability of unthreading is 1 and that any energy barrier for unthreading is small, noting that the barrier for threading is likely to be much larger than the barrier for unthreading ${ }^{\dagger}$.

\section{"Kink"-"Anti-Kink" Dynamics}

This algorithm is a novel way of dealing with long-ranged correlations introduced by the fact the chains are closed, and therefore have a well-defined topological state. Implementing these topological constraints is essential in simulating entangled ring polymers. The hindering of the motion when the terminal segments are threaded by another segment, i.e. forbidden to retract (Fig. B.2(e)) is taken into account via the following set of rules (one can visualise these in Fig. B.3 and Fig. B.2(e)). Starting from the configuration in Fig. B.3(a), when a move $m \rightarrow m^{\prime}$ is energetically accepted, a pair "kink" - "anti-kink" (or mass-hole) is generated at site $m$ (Fig. B.3(b)). While

\footnotetext{
${ }^{\dagger}$ We can actually generalise our interpretation by noting that the average number of penetrations will be determined by the ratio of probabilities (difference in energy barriers) between threading and unthreading, as usual. An alternative interpretation of $p_{t h}$ would therefore be the ratio of these rates, so that each value would correspond to the thermodynamically correct density of penetrations. The actual kinetic rate for unthreading, when the end of the ring has already diffused to the site of the threading, would still neglect the effect of a relatively small energy barrier. However, this is believed to be a tolerable simplification within a general philosophy that involves aggressive simplification, particularly given that the unthreading dynamics is likely dominated by the rate of diffusion of the penetrating portion of the double-folded ring, which can be much larger than a single unit. A small correction to the kinetic rate constant for the final unthreading step would then yield an even smaller correction to the overall result for the (un)threading dynamics. I therefore neglect it entirely for simplicity.
} 
the kink becomes instantaneously a new virtual segment $m^{\prime}$, the anti-kink starts a random walk from $m$ which can either hit $(i) m^{\prime}$, in which case the configuration goes back to the one in Fig. B.3.(a), (ii) a terminal end which is free (Fig. B.3.(d)), in which case the end is retracted and the new virtual segment $m^{\prime}$ becomes part of the chain which assumes the configuration sketched in Fig. B.3(e) or (iii) a terminal end which is pinned, in which case the end is not retracted, the move is rejected and the configuration goes back to the initial state (case shown in Fig. B.2(e)).

This algorithm allows for rejected moves caused by long ranged constraints introduced by the fact that the chains are closed and have to preserve their topological state. In other words, one can see this algorithm as describing elastic deformation of the chains which can protrude from any point along their contour, as opposite to linear polymers, for which the relaxation dynamics is strictly driven by their ends. In this case, the absence of ends forces the ring polymers to form hernias which protrude from any point along their backbone, given the uncrossability constraint is respected. These elastic deformations introduce a displacement of mass which can be described as a pair mass-hole or "kink"- "anti-kink'. While the kink describes the protrusion/extension attempted by the chain, the anti-kink probes the "availability" of ends which can be retracted, or free (not pinned) ends. This procedure represents a novel way of testing the entanglement of the chain. It has the advantage that it can take into account long-ranged constraints such as those represented by penetrations and hence it is sensitive to self-entanglements. In fact, the larger the number of pinned ends the more likely it is that a move is rejected.

This algorithm becomes identical to de Gennes' kink-gas diffusion in the limit $p_{t h}=0$, i.e. when no threading is allowed. On the other hand, in the case $p_{t h}>0$, I will show that this algorithm captures profoundly different behaviours which can shed some light onto the properties of self-entangled ring polymers.

Summarising, by using this algorithm, chains which can diffuse by extensionretraction of their segments are simulated. The retraction is constrained by the presence of self-threading segments which hinder the chain slithering. I implicitly assume that the relaxation of a single anti-kink is much faster than the extension of a new segment. The consequence of this is twofold: (1) only one "anti-kink" (or "hole") at the time is allowed to travel along the chain and (2) the time scale at which the motion of the chains takes place is the relaxation time of the "antikinks". In other words, the amoeba-like diffusion of the polymers at time scales larger than the "anti-kinks" diffusion is reproduced. This choice was made to give more emphasis on the long-time behaviour of the polymer dynamics.

It is also interesting to notice that this model naturally maps to a model for annealed branched polymers Iyer and Arya, 2012]. For $p_{t h}=0$ this model maps also to the well-established bond-fluctuation model for polymers Carmesin and Kremer, 1988, where the set of allowed bonds are restricted to preserve the topological state of the rings. From another point of view, one can notice that by setting $p_{\text {th }}=0$, we forbid the presence of loops in the configuration of the animals. In this case, this model is equivalent to a blob picture Obukhov and Rubinstein, 1994 for the double-folded rings, where the entanglement length is fixed to one lattice spacing. As $p_{t h} \rightarrow 1$, the probability of finding loops increases.

It is worth noting that only two beads are allowed on the same site at the same time. Hence, even when $p_{t h}=1$, the lattice animals are never completely ideal. Also, configurations in which two segments are sharing the same site but are not threading are not allowed. The motivation for this is the following: by coarse- 

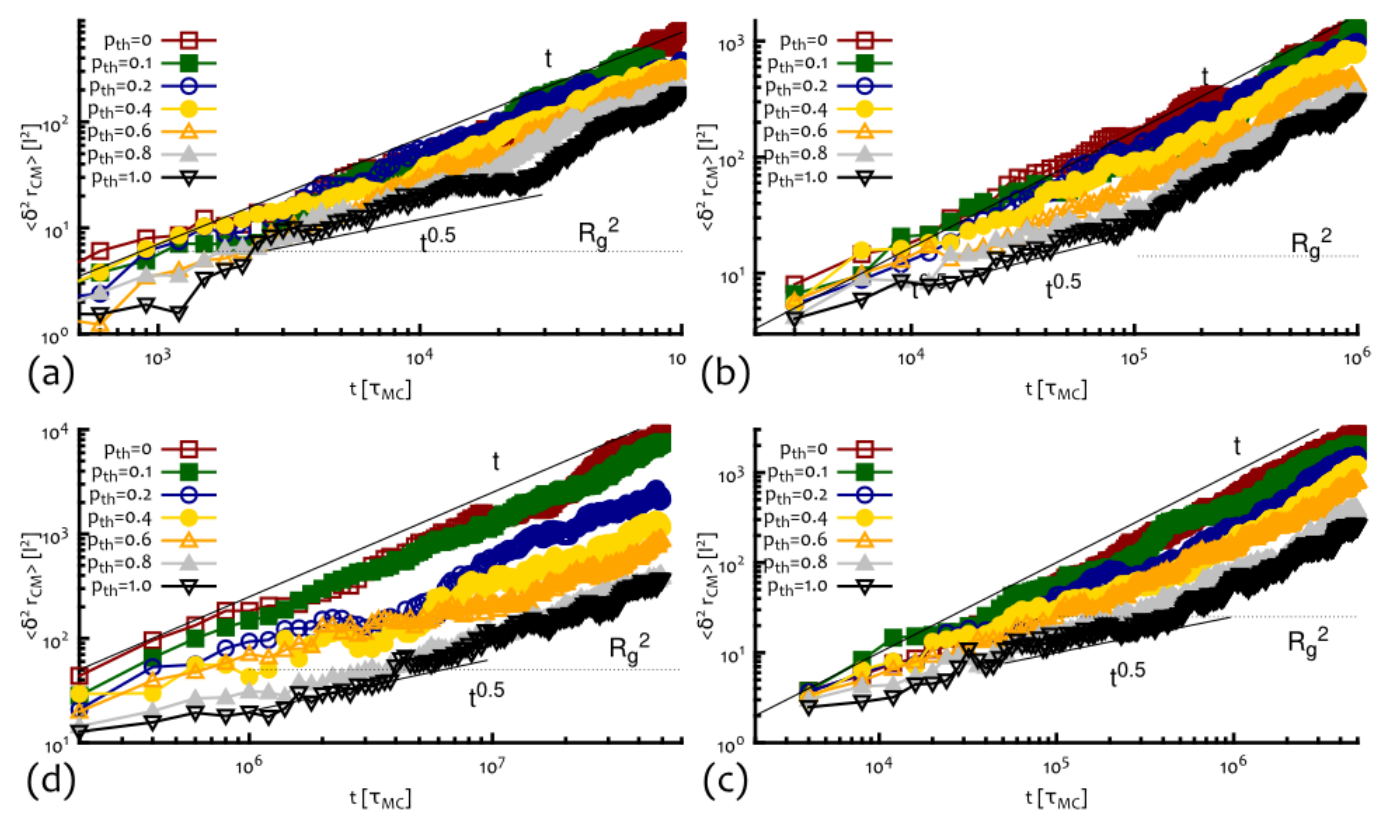

Figure B.4: Mean square displacement of the centre of mass of the rings as a function of time for different values of the threading probability $p_{t h}$ and increasing length $M$. It is worth noting the sub-diffusive behaviour $\delta^{2} r_{C M} \sim t^{x}$ with $x<1$ until intermediate times, even at moderate probability of threading $p_{t h}$. The slowing down can only be caused by an increasing number of self-threadings, which become more important for longer rings. In clockwise order: (a) $M=32$, (b) $M=64$, (c) $M=128$ and (d) $M=256$ beads.

graining the entire unit cell to one bead, the information on the local configuration inside each unit cell is lost and therefore is not possible to tell whether two chains sharing the same cell are threading or not. I arbitrarily choose to always label them as "threading". Making this choice implies that this model over-counts the number of self-threadings, however, I will show that the results clearly suggest that the macroscopic behaviour (scaling) of many observables is independent on the details of the model and especially the precise value of the free parameter $p_{t h}$, clearly demonstrating the importance of self-threading events on the motion of long ring polymers.

\section{Results}

\section{Diffusion Coefficient}

By tuning the free parameter $p_{t h}$ the effect of self-threading events on the motion of the polymers can be studied. In the case $p_{t h}=0$ the chains are expected to follow a pure amoeba-like diffusion, where the diffusion of "anti-kinks" takes a time of order $\tau_{\text {kink }} \sim M^{2}$ to travel a distance $R_{g} \sim M^{1 / 2}$ Parisi and Sourlas, 1981, Cates and Deutsch, 1986. Since at every time step, all $M$ segment attempts to move, on average, the expected time taken for the centre of mass to diffuse a distance $R_{g}$ scales as

$$
T_{r}\left(p_{t h}=0\right) \equiv T_{0}=M \tau_{k i n k} \sim M^{3}
$$


and consequently, the diffusion coefficient of the centre of mass of an isolated chain in gel is:

$$
D_{C M}\left(p_{t h}=0\right) \equiv D_{0}=\frac{R_{g}^{2}}{T_{0}} \sim M^{2 \nu-3}=M^{-2}
$$

since $\nu=1 / 2$ in 3d for self-avoiding rings in gel Parisi and Sourlas, 1981. The results are incidentally the same as for reptating linear polymers, as observed in the literature Cates and Deutsch, 1986]. This is due to the fact that the exponent $\nu$ for self-avoiding lattice animals coincides with the value for Gaussian chains. The mean square displacement of the centre of mass $\left\langle\delta^{2} r_{C M}\right\rangle$ (see Ch. 2) as a function of time and for different values of the chains length and probability of threading in reported in Fig. B.4. Since the model does not capture the dynamics at time-scales shorter than the kink relaxation time, we expect to observe pure free diffusion of the centre of mass of the rings in the case $p_{t h}=0$. In fact, as one can notice, a free diffusive behaviour throughout the time window is obtained for $p_{t h}=0$. On the other hand, for $p_{t h} \rightarrow 1$ the mean square displacement of the centre of mass shows sub-diffusive behaviour at intermediate times with crossover to free diffusion only at longer times. This behaviour is unambiguously related to the presence of self-threadings, since the system is in the dilute regime. In other words, allowing the rings to self-thread results in an increasingly important self-constraint on the motion. These contributions on the motion are ultimately caused by the preservation of the topological state of the rings, which have to stay unknotted and unlinked from both the gel and themselves.

The length-scales associated with the crossover from sub-diffusive behaviour to the free diffusive one lie between $\left(R_{g}^{2}\right)^{1 / 2}$ and $\left(10 R_{g}^{2}\right)^{1 / 2}$. This feature is in agreement with previous findings in systems of rings with similar topological constraints (Ref. Halverson et al., 2011b and Ch. 4). These length-scales are argued to be intimately related with the loss of threadings, and that the rings have to travel many times their own average size before relaxing all the threadings, i.e. freely diffusing. It is worth noting that linear or branched polymers would not undergo the same change in diffusion by allowing sites with double occupancy, as there is no defined topological state to be conserved. The feature I am reporting in Fig. B.4 is peculiar of systems made of polymers with closed topology and in which the uncrossability constraint is taken into account.

In Fig. B.5 the scaling behaviour of the diffusion coefficient of the centre of mass $D_{C M}\left(p_{t h}\right) \equiv \lim _{t \rightarrow \infty}\left\langle\delta^{2} r_{C M}\right\rangle / 6 t$ is shown. Notice that $D_{C M}(0) \equiv D_{0} \sim M^{-2}$, in perfect agreement with the scaling regime obtained for the pure (un-constrained) amoeba-like motion Rubinstein, 1986, Cates and Deutsch, 1986, Obukhov and Rubinstein, 1994. For higher values of $p_{t h}$ the rings diffuse slower, due to the chains' self-threading. The $M$ dependence of the diffusion coefficient of the centre of mass of the rings is observed to become more severe as $p_{t h} \rightarrow 1$.

\section{Radius of Gyration}

In order to obtain the scaling law governing the gyration radius of the polymer, the quantity $\left\langle R_{g}^{2}\right\rangle^{1 / 2}$ (see Sec. 2 is measured over statistically independent ring polymer configurations. The results are shown in Fig. B.6.

The radius of gyration at $p_{t h}=0$ is in agreement with the mean-field prediction $\left(\left\langle R_{g}^{2}\right\rangle \sim M\right)$ for lattice animals with excluded volume Lubensky and Isaacson, 1979, Parisi and Sourlas, 1981, Daoud and Joanny, 1981. Higher values of $p_{t h}$ allow 


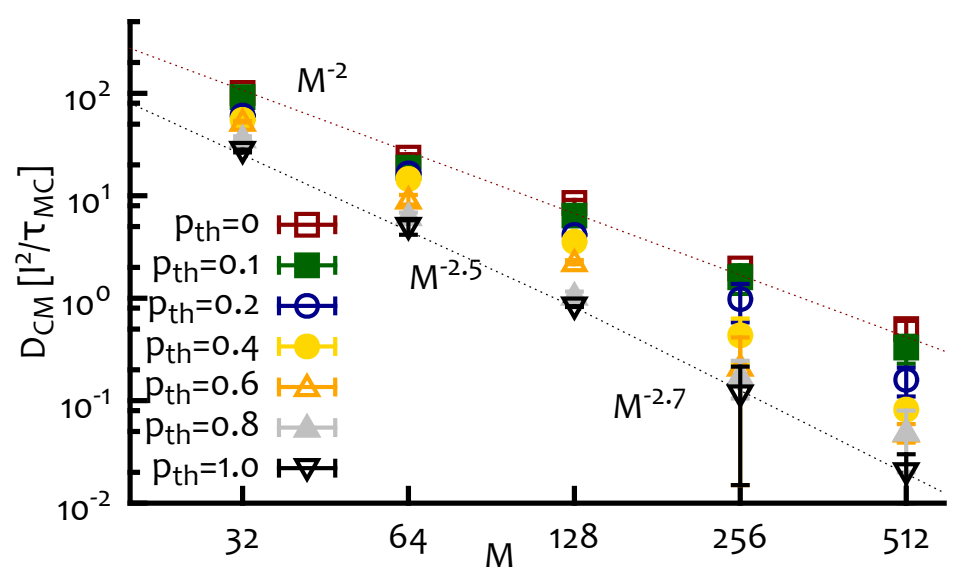

Figure B.5: Plot showing the diffusion coefficient of the centre of mass $D_{C M}$ computed as $\lim _{t \rightarrow \infty} \delta^{2} r_{C M} / 6 t$ as a function of the chains length $M$. One can observe pure amoeba-like (free) diffusion at $p_{t h}=0$, which scales as $D_{C M} \sim M^{-2}$ according to eq. (B.2), and slower diffusion $D_{C M} \sim M^{-\alpha}$ with $\alpha>2$ for increasing $p_{t h}$.

the polymers to partially self-overlap (only a maximum of two beads per site are allowed) and therefore reduce their size. The mean-field prediction for ideal (non self-avoiding) lattice animals Lubensky and Isaacson, 1979 $\left(R_{g} \sim M^{1 / 4}\right)$ breaks down at $d<d_{c}$ with $d_{c}=8$ for lattice animals in good solvent, therefore this is not expected to be valid in the system under study. The scaling behaviour at $p_{t h}=1$ resembles instead a regime in which the lattice animals have screened out two-body excluded volume interactions, i.e. the (repulsive) second virial coefficient is zero, while they retain three-body excluded volume. It has been recently conjectured that the gyration radius of rings in the melt, where two-body interactions are screened by the high density of the solution, assume the minimum value compatible with general excluded volume constraints, i.e. $\left\langle R_{g}\right\rangle \sim M^{1 / 3}$ in $d=3$ Grosberg, 2014, Rosa and Everaers, 2014. This is compatible with the findings presented here (see Fig. B.6(a)), where one can think the two-body repulsion as increasingly screened by tuning $p_{t h}$ to values close to 1 .

The functional dependence of the exponent $\nu$ on the value of the free parameter $p_{t h}$ is also investigated (see Fig. B.6(b)). The function that best fits the data is a sigmoid function which continuously crossovers from a lattice animal value of $\nu=1 / 2$ to a more compact value of $\nu=1 / 3$ as $p_{t h}$ crosses $p_{t h}^{*} \simeq 0.5$. This model captures such crossover by only varying the free parameter $p_{t h}$. Under this perspective, it is clear that $p_{t h}$ plays the role of an effective second virial coefficient, which regulates two body repulsion. It also worth noting that for any polymer whose topology is nonclosed, a variation of the second virial coefficient is not expected to affect its motion as severely as one can observe in the case of rings. At the heart of this feature there is the preservation of the topological state, via the uncrossability condition. In fact, even though the two-body repulsion is indirectly regulated by the parameter $p_{t h}$, and gradually turned off when $p_{t h}$ approaches unity, the uncrossability constraint that forbids any real strand-crossing is always preserved. In light of this, in the next section I will investigate the statistics of self-threadings and their functional dependence on length $M$ and probability of threading $p_{t h}$. 


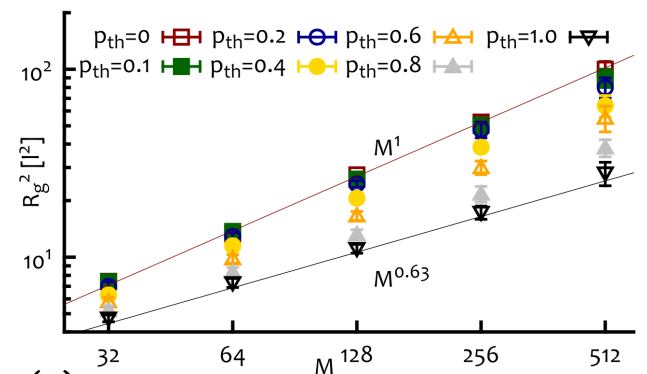

(a)

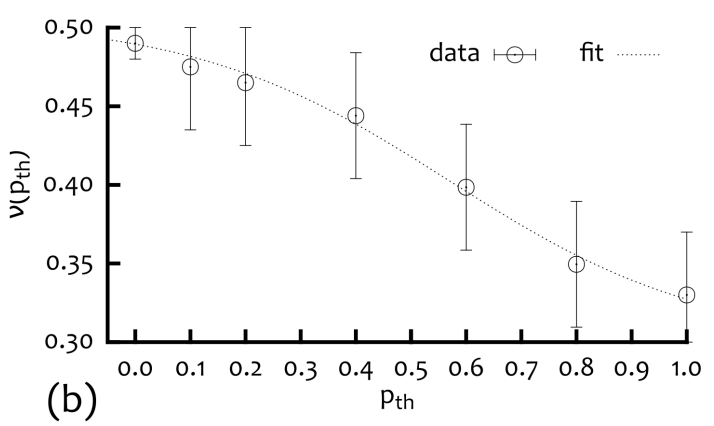

(b)

Figure B.6: (a) Radius of gyration of the chains as a function of the rings length $M$. The prediction $R_{g} \sim M^{1 / 2}$ for ring polymers in gel is obtained at $p_{t h} \rightarrow 0$ Parisi and Sourlas, 1981]. For $p_{t h} \rightarrow 1$ the pairwise repulsion of segments is effectively sent to zero, and one can consequently observe $R_{g} \rightarrow M^{1 / d}$, as predicted in the literature Daoud and Joanny, 1981, Grosberg, 2014, Rosa and Everaers, 2014. (b) Values of the exponent $\nu$ as a function of $p_{t h}$ and $M=256$. The fit has been performed using a sigmoid function $\nu(x) \sim A /\left(1+e^{w\left(x-p_{t h}^{*}\right)}\right)+B$. The result suggests a continuous transition from lattice animal behaviour $(\nu=1 / 2)$ to fractal globule behaviour $(\nu=1 / 3)$ as $p_{t h}$ crosses $p_{t h}^{*} \sim 0.5$.

\section{Threadings and Pinned Ends}

In order to establish a clear correspondence between the uncrossability constraint and the slowing down of the rings dynamics, I keep track of the self-threading events and report their number as a function of the rings length, $M$ (see Fig. B.7). The number of self-threadings per chain is found to scale extensively with $M$ times a constant that in general can depend on $p_{t h}$, i.e. $\langle T h\rangle \sim A\left(p_{t h}\right) M$. As one can see from Fig. B.2, a self-threading looks very similar to a loop in the polymer conformation. Previous works confirmed that the number of loops in lattice animal conformations does not represent a critical quantity Lubensky and Isaacson, 1979, Daoud and Joanny, 1981, i.e. their number remains constant as the length of the animals increases. I would like to point out that, in this model, self-threadings are not fully equivalent to loops as intended in some literature Lubensky and Isaacson, 1979. One can see this by looking at Fig. B.2(e). As one segment threads through another, the functional unit of the interacting beads is preserved, i.e. the two ends threading through each other in Fig. B.2(e) remain distinct ends. This is computationally implemented by stacking the beads on top of each other, rather than merging the two beads together. In other words, self-threading is not equivalent to a polymerisation process, but instead is reversible via purely dynamical moves.

As a consequence, the critical nature of the threadings, which are here shown to grow linearly with $M$ (Fig. B.7), is not in disagreement with the literature Lubensky and Isaacson, 1979]. For every value of $p_{t h}>0$ one can observe that the number of threadings is extensive in $M$, i.e. $\langle T h\rangle \sim M$, in the large $M$ limit. This result clearly states that, independently on the precise value of the parameter $p_{t h}$, an abundant number of self-threading events will emerge as the length of the rings increases, ultimately hindering the motion of the rings by restricting the free retraction of more and more ends.

In Fig. B.8(b) the fraction of free ends, i.e. ends that are not pinned and can contribute to the rings motion, over the total number of ends of a lattice animal is shown. The number of pinned ends is easily obtained as $\left\langle N_{e}\right\rangle=\left\langle N_{f e}\right\rangle+\left\langle N_{p e}\right\rangle$. The 


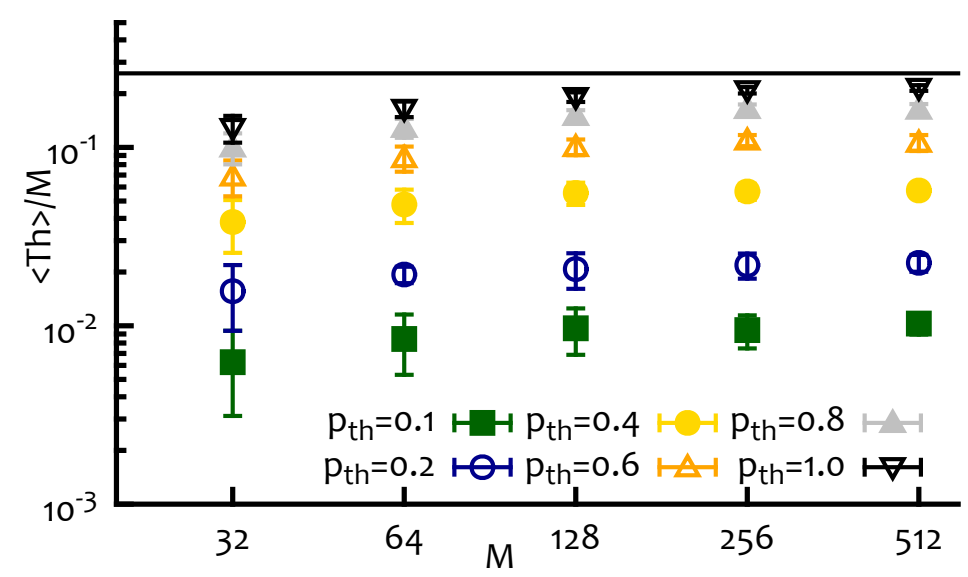

Figure B.7: Plot showing $\langle T h\rangle / M$ as a function of the chains length $M$. For large $M$, the number of threadings per chain is shown to scale extensively in $M$ regardless the precise value of $p_{t h}$, which strongly suggests a universal and broad validity of the findings described in this Chapter.

total number of ends in a lattice animals is observed to be extensive in its length $M$ (not shown). Of these, a fraction $\left\langle N_{f e}\right\rangle /\left\langle N_{e}\right\rangle$ is free to retract, while a fraction $1-\left\langle N_{f e}\right\rangle /\left\langle N_{e}\right\rangle$ is threaded, or pinned, and hence not free to retract. In Fig. B.8 it is shown that as the length of the rings increases, the fraction of free ends decreases and this effect is more evident as $p_{t h}$ gets closer to 1 .

\section{Relaxation Dynamics}

The slowing down due to self-threading of the chain is apparent from the plot of the relaxation time $T_{r}$ of the chain in Fig. B.8(a). This quantity gives a measure of how long it takes for a chain to diffuse a distance comparable to its gyration radius and it is computed as $T_{r}=\left\langle R_{g}^{2}\right\rangle / D_{C M}$, which can in general depend on both, $p_{t h}$ and $M$. One can observe that the relaxation time dramatically increases with chain size, with a power law which appears to depend on $p_{t h}$. At $p_{t h}=0$, the relaxation time is compatible with the theoretical prediction of eq. (B.1), while for higher values of $p_{t h}$ it shows to be more strongly dependent on the chain length. In Fig. B.8.(c) the relaxation time $T_{r}$ as a function of the fraction of pinned ends $\left\langle N_{p e}\right\rangle /\left\langle N_{e}\right\rangle$ is shown. One can observe that, compared to the value at $p_{t h}=0$, the relaxation time at $p_{t h}>0$ can be substantially larger. Even at moderate values of $p_{t h}$ one can observe a significant slowing down. For instance, for $M=512$ (uppermost row of symbols in Fig. B.8(c)), one can notice that $T_{r}\left(p_{t h}=0.4\right)$ (yellow dotted line) is roughly five times larger than the relaxation time at $p_{t h}=0$ (red dotted line), which could easily be observed experimentally.

\section{Relaxation of the Threadings}

In order to further quantify the effect of the threadings on the long-time dynamics of the rings, I investigate the resistance of the threadings by taking fully equilibrated configurations with $p_{t h}>0$, and turning the free parameter $p_{t h}$ to zero. In other words, we forbid the creation of new pinned sites and study the time-scale required for the self-threadings to fully relax. This is quantified by measuring the equivalent of the stress relaxation modulus for threadings $G_{t h}(t)$. This is here computed by 

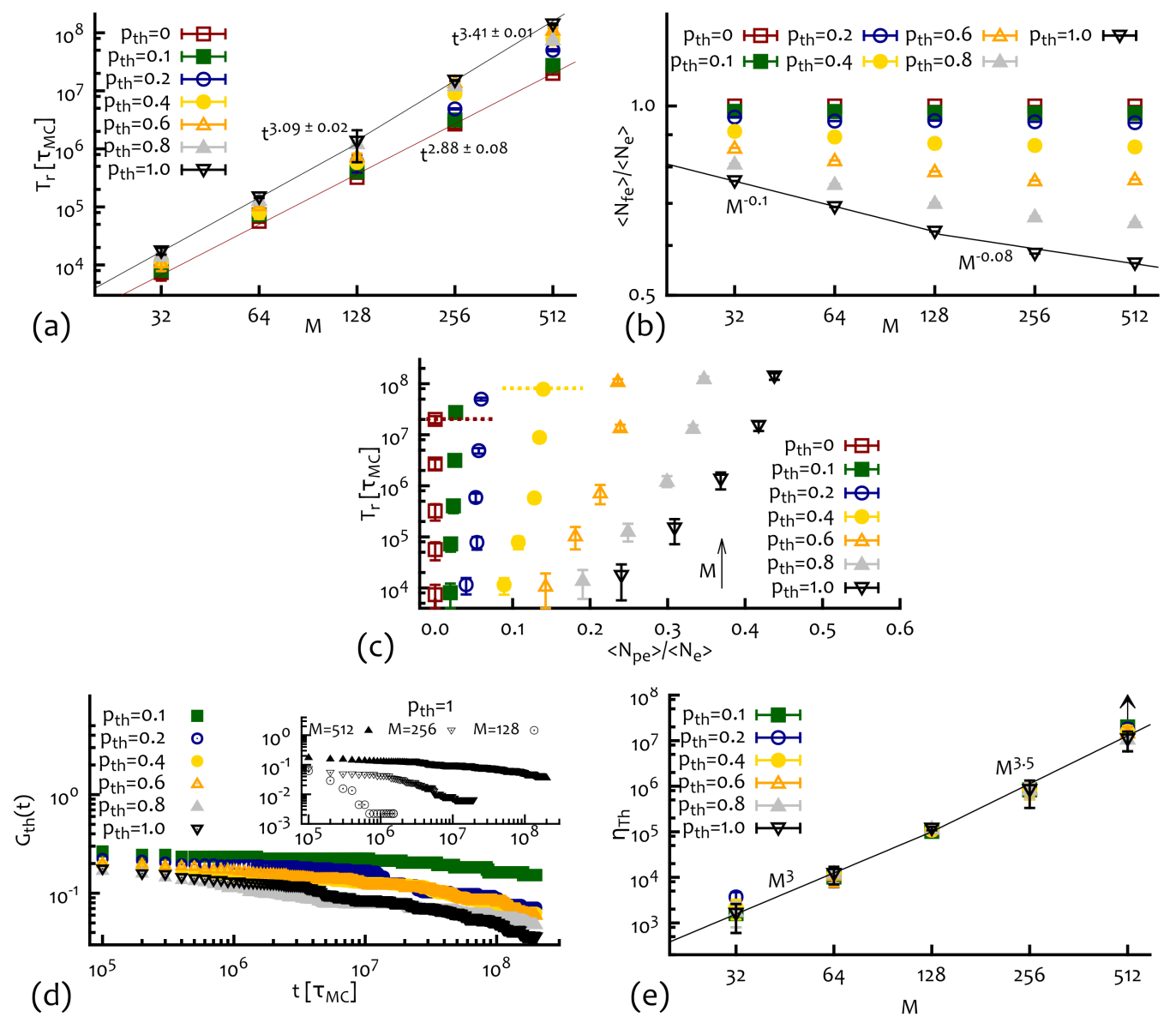

Figure B.8: (a) Plot showing the relaxation time $T_{r}=\left\langle R_{g}^{2}\right\rangle / D_{C M}$ as a function of the rings length $M$. The prediction for amoeba-like motion, i.e. $T_{r} \sim M^{3}$ is compatible with the findings for $p_{t h}=0$. For higher values of $p_{t h}$ one can observe a slowing down which can shed some light onto the motion of entangled rings in gel. (b) Plot showing the ratio $\left\langle N_{f e}\right\rangle /\left\langle N_{e}\right\rangle$ as a function of the chains length. The curve for $p_{t h}=1$ seems to approach the limiting value of $1 / 2$, at which half of the ends would be pinned and cannot contribute to the motion. (c) Log-linear plot showing the relaxation time $T_{r}$ as a function of the fraction of pinned ends. The length of the rings $M$ increases upwards. Notice that even for moderate values of $p_{t h}$ we obtained substantial slowing down. For $M=512$ the relaxation time $T_{r}$ at $p_{t h}=0.4$ (yellow dotted line) is five times larger than $T_{r}$ at $p_{t h}=0$ (red dotted line). (d) The threading relaxation function $G_{t h}(t)$ as a function of time for different $p_{t h}$ and different chain lengths (inset). (e) The zero-shear viscosity $\eta_{t h}$ shows a power law behaviour $\tau_{t h} \sim M^{\alpha}$, with $\alpha=3$ for $M<128$ and $\alpha=3.5$ for $M>128$. The arrow at $M=512$ indicates that the values of $\eta_{t h}$ for the longest rings represent only a lower bound as we could not observe the removal of all the threadings within the simulation runtime.

considering a vector at time $\tau, \boldsymbol{t h}(\tau)$, whose entries are 1 and having a length equal to the total number of self-threadings at time $\tau$. At every successive time-step a new vector $\boldsymbol{t h}(\tau+t)$ is created where the length is kept constant but its $i$-th entry is 1 if threading $i$ is present at that time-step, or 0 otherwise. The relaxation of threadings is then computed by taking the dot product of these vectors at successive time-steps, opportunely normalised and averaged over rings and realisations, i.e.

$$
G_{t h}(t)=\left\langle\frac{\boldsymbol{t h}(\tau+t) \cdot \boldsymbol{t h}(\tau)}{\boldsymbol{t h}(\tau)^{2}}\right\rangle .
$$


In other words, this represents the average fraction of self-threadings present $t$ timesteps after the self-threading parameter $p_{t h}$ is set to zero.

In the case the self-threadings were uncorrelated with each-other, one would expect $G_{t h}(t)$ to follow a Poisson process with a certain rate, which could in general depend on the average length of the threading segment. In other words, one would expect an exponential decay of $G_{t h}(t)$. While this could approximate the behaviour observed for short chains (inset Fig. B.8(d)), a strong deviation from this behaviour is observed when longer chains are considered. After an initial drop that brings $G_{t h}(t)$ from 1 to roughly 0.3 , one can observe a very slow power law decay on the time-scales comparable with the chains longest relaxation times (see Fig. B.8(d)). This suggests that while short and uncorrelated threadings are quickly released at short time-scales, for long chains there exist a structure of correlated threadings which have to be released in a precise manner in order to reach full relaxation. This suggests that self-threadings create a nested network of constraints that lasts for several decades and is significant on the time-scales of the rings motion. For the longest rings, the removal of all the threadings could not be observed even at very long times (see Fig B.8).

The equivalent of the zero-shear viscosity for the threadings is computed as the numerical integral of $G_{t h}(t)$, i.e. $\eta_{t h}=\int_{0}^{\infty} G(t) d t$ (see Fig. B.8(e)). Its value seems to be insensitive on the value of $p_{t h}$ and to scale as $\eta_{t h} \sim M^{\alpha}$, with $\alpha \geq 3$. For the longest rings, we could compute only the lower bound of $\eta_{t h}$, as $G_{t h}(t)$ does not show a decay to a negligible value within the simulation window. This is indicated in Fig. B.8(e) by the arrow coming out the data points at $M=512$. The exponents observed for this quantity are closer to the experimentally reported value of 3.9 Tead and Kramer, 1992 than other models of rings moving in gels found in the literature [Obukhov and Rubinstein, 1994, Rubinstein, 1986]. This strongly encourages the fact that self-threadings represent long-lived correlations on the chains motion which can be responsible for the slowing down of ring polymers diffusion in gels.

\section{Remarks}

In summary, I presented a Kinetic Monte-Carlo algorithm which simulates a dilute solution of ring polymers in a gel. Under certain conditions, i.e. $p_{t h}=0$, this algorithm reproduces the well known theoretical results for un-constrained rings in gel Obukhov and Rubinstein, 1994, Cates and Deutsch, 1986. On the other hand, it also adds the possibility of taking into account self-threadings: the static and dynamic properties of the rings have been studied by tuning the free parameter $p_{t h}$. A drastic change in the polymers behaviour is observed as $p_{t h} \rightarrow 1$ (see Fig. B.4). The severe slowing down observed in the polymers dynamics is caused by the presence of long-ranged and long-lived correlations which take the form of self-threadings (see Figs. B.5 and B.8). Such constraints on the polymers diffusion are intimately related to the fact that the polymers have a closed topology. Even for moderate values of $p_{t h}$ the relaxation time of the rings can be almost one order of magnitude larger than the case without threadings (see Fig. B.8).

More importantly, and possibly the most important results of this Section, is that the number of these self-threadings is found to scale extensively with the size of the chains $M$, regardless on the precise value of the free parameter $p_{t h}$ which strongly encourages that (1) the results presented here have a general character and 
are insensitive on the microscopic details of the system and (2) that the threadings are expected to be be prolific as long as the chains considered are long enough (see Fig. B.7).

It is worth noting that the effects of self-threadings on the rings dynamics are expected to become distinctly measurable experimentally only for gels that do not contain dangling ends, as in the case of micro-lithic arrays Volkmuth and Austin, 1992. Duke et al., 1996. Isolating the effect of the slowing down caused by "impalement" of the rings (Ref. [Viovy, 2000] and Ch. 6) from that caused by self-threadings can be difficult experimentally. It is worth reminding that one can think of $p_{t h}$ as an effective second virial coefficient, i.e. it regulates two-body repulsion. In light of this, $p_{t h}$ might be directly controlled by acting on the temperature, where conditions of $\theta$-temperature or poor solvent are expected to facilitate the occurring of self-threading events. In general, in the case of good solvents, the value of $p_{t h}$ is expected to be small, hence self-threadings are conjectured to represent a real hindering on the dynamics only of very large polymers, i.e. of order of thousands of Kuhn segments. This translates into, for instance, DNA plasmids $100 \mu \mathrm{m}$ or 300 kbp long Thomas and Summers, 2009. 


\section{$\mathrm{C}$ The Replication of KDNA}

In this appendix I will briefly describe a effective 1D model for the replication of the Kinetoplast DNA. One of the most puzzling and interesting aspects of its replication is that it generates the same network structure at each replication cycle with very few, or none, mistakes [Jensen and Englund, 2012]. The cell viability requires a key feature: the genetic information contained in the Kinetoplast has to be passed on to the progeny. This means that either quantity and quality of the genetic material have to be conserved.

In Ch. 5 I have shown that it can be argued that the percolating network structure of the Kinetoplast could have been evolved in order to meet the need to conserve the quantity of genetic material. The percolating nature of the network can in fact be though of as a natural mechanism through which these organisms ensure that most of the mini-circles belong to a unique component and therefore are not free to diffuse away from the Kinetoplast. On the other hand, the linkedness of the network might not be beneficial to the cell during replication as in fact it could hinder the duplication process. How the Kinetoplast is duplicated and divided into the progeny is, in fact, far from being well understood Chen et al., 1995a, Jensen and Englund, 2012 , and this is mainly due to its complexity. In spite of this, from a bio-physical perspective, the duplication of the Kinetoplast can be thought of as made of three major stages which correspond to three topological changes in the network structure. As a consequence, in what follows I will introduce a minimal analytical model that focuses on these three steps and attempt to get some insight into this complicated issue by aggressively simplify the problem, in the same spirit of Ch. 5 .

\section{Biological Details and Assumptions}

As mentioned before, the replication of the Kinetoplast involves three major stages:

( $i$ ) the removal of mini-circles from the network via the action of Topoisomerase II Shlomai, 1994],

(ii) the free diffusion of the un-linked mini-circles away from the Kinetoplast core and toward the anti-podal regions where they are duplicated Jensen and Englund, 2012 


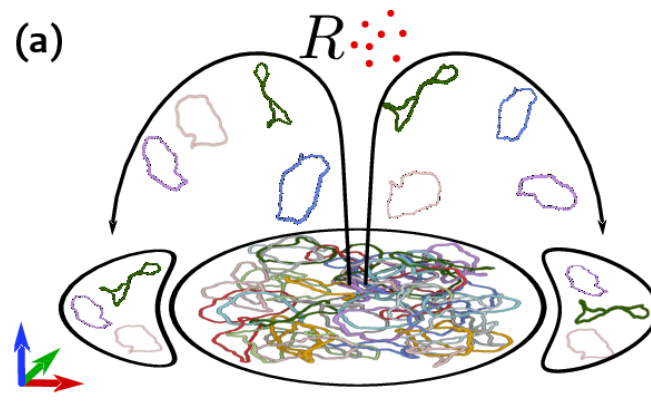

(b)

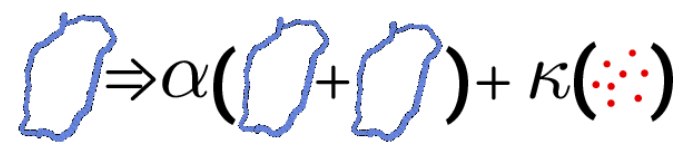

(c)
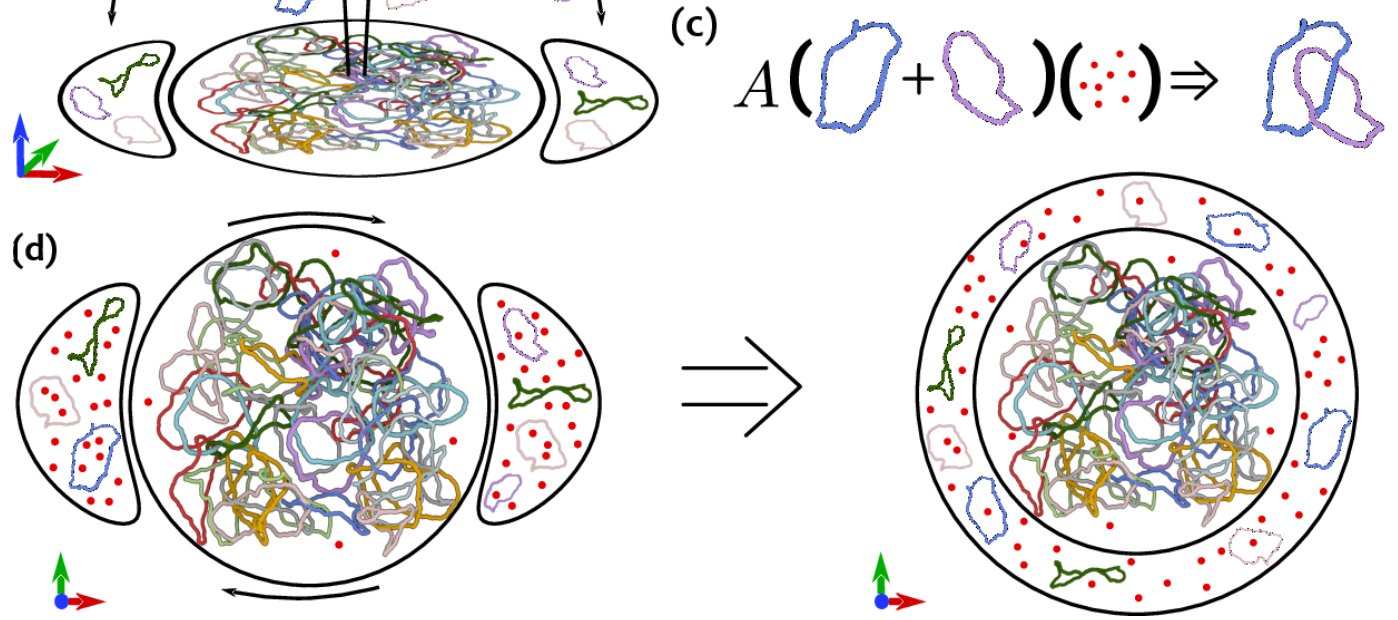

Figure C.1: (a) Graphical representation of the reaction in eq. (C.1). Topoisomerase (red dots) unlinks mini-circles (coloured loops) from the central connected network. (b) Graphical representation of the reaction in eq. (C.2). Each mini-circle duplicates at rate $\alpha$ and indirectly triggers the downstream production of Topoisomerase (red dots) at rate $\kappa$. (c) Graphical representation of the reaction in eq. (C.3). Each mini-circle forms a catenane with another mini-circle (either free or linked) at a rate proportional to the local concentration of Topoisomerase. (d) The model is further simplified by substituting the two anti-podal sites where re-attachment occurs with a shell; this can be thought of as an average over the relative position of the (rotating) Kinetoplast core with respect to the anti-podal sites within the replication time-window.

(iii) the re-attachment of the progeny mini-circles to the periphery of the network [Pérez-Morga and Englund, 1993b].

These three conditions are necessary for the network replication and the organism viability. These stages can be translated in three pseudo-equations as follows:

(i) Each mini-circle is unlinked from its linked neighbours at a rate that is proportional to the local concentration of Topoisomerase II $\left(\phi_{T}\right)$ (see Fig. C.1(a)):

$$
(N) \text { Linked } \stackrel{R \phi_{T}}{\longrightarrow} \text { Unlinked }+(N-1) \text { Linked } .
$$

(ii) While diffusing, each mini-circle begins its duplication thanks to polymerase enzymes present in the kinetoflagellar zone around the Kinetoplast Morris et al., 2001, Drew and Englund, 2001. This process occurs at a rate $\alpha$ which is regulated by the speed at which polymerases duplicate the genetic sequence. Mini-circles can only be transcribed at this stage alongside to their duplication, i.e. while their are unlinked from the network. This is supported by the fact that transcription enzymes cannot easily enter the depths of the compact network structure, and even in the case they would, the entanglement caused by the density of material would strongly suppress any transcriptional activity. In other words, I assume that the genetic information contained in the mini-circles is more easily and readily accessible at the stage when they are free from the network.

Relying on the well established fact that mini-circles contain RNA-editing genes 
Jensen and Englund, 2012, I further assume that mini-circles transcription triggers, among other things, the downstream production of topoisomerase II at a rate $\kappa$ via transcription and RNA-editing factors encoded in the mini-circles genetic material (see Fig. C.1(b)). This assumption is supported by RNA interference (RNAi) experiments [Wang et al., 2000], in which the expression of genes encoding for topoisomerase II and mitochondrial mRNA synthesis are inhibited, causing loss of Kinetoplast and cell death. In pseudo-equations this stage reads:

$$
\begin{aligned}
& \text { Unlinked } \stackrel{\alpha}{\rightarrow} \text { Unlinked }+ \text { Unlinked } \\
& \text { and } \\
& \text { Unlinked } \stackrel{\kappa}{\rightarrow} \text { Topoisomerase. }
\end{aligned}
$$

(iii) Once the progeny mini-circles diffuse to the anti-podal sites, they are religated by gap-filling enzymes Pérez-Morga and Englund, 1993a and re-attached at the periphery of the network. The re-attachment rate can be thought again proportional to the local concentration of topo II $\left(\phi_{T}\right)$ Wang and Englund, 2001 (see Fig. C.1(c)):

$$
\begin{aligned}
& \text { Unlinked }+ \text { Unlinked } \stackrel{A \phi_{T}}{\longrightarrow} \text { Linked } \\
& \text { or } \\
& \text { Unlinked }+ \text { Linked } \stackrel{A \phi_{T}}{\longrightarrow} \text { Linked }
\end{aligned}
$$

where the pseudo-equations represent the cases in which the free mini-circle is relinked to an other un-linked or an already linked mini-circle, respectively. Finally, the rates $R$ and $A$ are key parameters in this model and represent the rates of removal $(R)$ and re-attachment $(A)$ of mini-circles from and to the network.

A final comment on the model is in order: During the replicating phase of $C$. fasciculata, the Kinetoplast core has been observed to rotate relatively to the anti-podal sites where re-attachment occurs Pérez-Morga and Englund, 1993b. In light of this, and of the inherent rotational symmetry of the final replicated network Pérez-Morga and Englund, 1993b, Jensen and Englund, 2012, one could argue that effectively, the two anti-podal complexes can be replaced with a shell that surrounds the Kinetoplast core (see Fig. C.1(d)). This substitution is equivalent to perform a time-average of the relative position of the complexes with respect to the core over the whole replicating phase. The entire system can be visualised as a rotationally symmetric 2-dimensional disk divided into a core $r \leq R_{k}$ filled by the Kinetoplast at the beginning of the replication $(t=0)$ and a shell $R_{k}<r<R_{\max }$ (initially empty of genetic material) which plays the role of region where re-attachment occurs.

\section{Modelling Topological Changes}

The three stages are therefore related to three topological operations: $(i)$ un-linking of mini-circles from the network, (ii) duplication of single mini-circles and (iii) linking of the progeny mini-circles back to the network. These are captured by the pseudo-equations (C.1)-(C.3).

One can now argue that the system could be also described in terms of the relative density of linked $\rho_{l}(\boldsymbol{x}, t)$ and unlinked $\rho_{u}(\boldsymbol{x}, t)$ mini-circles and of topoisomerase II enzyme $\phi_{T}(\boldsymbol{x}, t)$. At the beginning of replication, the (normalised) density of linked mini-circles is 1 within the Kinetoplast core $\left(\rho_{l}(\boldsymbol{x}, 0)=1\right.$ for $|\boldsymbol{x}| \leq R_{k}$ and 0 
otherwise), and there are no unlinked mini-circles $\left(\rho_{u}(\boldsymbol{x}, 0)=0 \forall \boldsymbol{x}\right)$. During the replicating phase, the total number of mini-circles grows and at the end of the replication the total number of mini-circles is twice the initial number, i.e. $\rho_{l}^{*}+\rho_{u}^{*}=2$, where $\rho_{l}^{*}$ and $\rho_{u}^{*}$ are the final (uniform) values of $\rho_{l}$ and $\rho_{u}$, respectively.

By describing the system in terms of these local densities, or "fields", one can translate eqs. C.1 - C.3 into three coupled equations:

$$
\begin{aligned}
\frac{d \rho_{l}}{d t} & =-R \rho_{l} \phi_{T}+A \phi_{T} \rho_{u}\left(\rho_{l}+\rho_{u}\right)+D_{l} \nabla^{2} \rho_{l} \\
\frac{d \rho_{u}}{d t} & =+R \rho_{l} \phi_{T}-A \phi_{T} \rho_{u}\left(\rho_{l}+\rho_{u}\right)+D_{u} \nabla^{2} \rho_{u}+\alpha \rho_{u}\left[2-\left(\rho_{u}+\rho_{l}\right)\right] \\
\frac{d \phi_{T}}{d t} & =+\kappa \rho_{u}-\frac{\phi_{T}}{\tau}+D_{T} \nabla^{2} \phi_{T},
\end{aligned}
$$

where the first and second terms in eqs. (C.4)- (C.6) capture the reactions represented by eqs. (C.1)-C.3 and the last term in eq. C.5 ensures that the un-linked minicircles duplicate at rate $\alpha$ until the sum $\rho_{u}+\rho_{l}$ reaches 2 , at which point the growth is stopped, while the third terms describe the usual diffusion of mini-circles and topo II with their respective diffusion constants.

The value of $\alpha$ at which mini-circles are duplicated can be estimated by noting that polymerase replicates DNA at a speed between $20 \mathrm{bp} / \mathrm{s}$ (in bacteria) and 500 $b p / s$ (in eukaryotes) Dignam et al., 1983. Wickiser et al., 2005, Schwartz and Quake, 2009. Therefore the typical duplication time results to be of about $\alpha^{-1} \simeq 2000$ $\mathrm{bp} /(100 \mathrm{bp} / \mathrm{s})=20 \mathrm{~s}$.

In addition, the rate of (indirect) production of topo II is set as $\kappa=\alpha=0.05 \mathrm{~s}^{-1}$. This choice is justified by the fact that the transcription of mini-circles is expected to take a time comparable to their duplication. Finally, the decay time $\tau$ associated to topoisomerase enzymes can be set to $\tau=600 \mathrm{~s}$, being this its typical half-life in bacteria Taniguchi et al., 2010].

Since unlinked mini-circles are freed from the network, they are expected to diffuse away from the Kinetoplast core. Previous studies have reported that mini-circle duplication begins in the Kinetoflagellar Zone (KFZ), a dense matrix of filaments which attaches the Kinetoplast to the flagellar basal body Ogbadoyi et al., 2003, surrounding the Kinetoplast and filled with Polymerase enzymes, and it is always completed inside the anti-podal regions, where a high concentration of gap-filling enzymes has been found Jensen and Englund, 2012]. The diffusion coefficients, $D_{l}$, $D_{u}$ and $D_{T}$ regulate the diffusive behaviour of, linked and unlinked mini-circles and of topo II enzymes, respectively.

In other words, the values of all the parameters (a part from $A$ and $R$ ) in eqs. (C.4)-(C.6) can be directly informed by biological evidence. The rate of attachment $A$ and of removal $R$ are therefore the only true free parameters of this model and possibly the most difficult to study experimentally.

\section{The Stable Point is a Marginally Linked Network}

Eqs. C.4 - C.6 are most readily studied numerically, also thanks to the rotational symmetry of the problem (see Michieletto et al., 2014). Here I will instead study these equations analytically.

Eqs. (C.4)-C.6 correctly describe an unstable fixed point of the system when 
topo II is depleted from the system. In other words, the state

$$
S(t=0)=\left\{\begin{array}{l}
\rho_{l}(\boldsymbol{x}, 0)=1 \forall \boldsymbol{x} \text { s.t. }|\boldsymbol{x}| \leq R_{k} \\
\rho_{u}(\boldsymbol{x}, 0)=0, \phi_{T}(\boldsymbol{x}, 0)=0 \forall \boldsymbol{x}
\end{array}\right.
$$

satisfies eqs. (C.4)-C.6) and is a fixed point of the system. I would like to stress that this state can be truly thought of as the biological initial state of the Kinetoplast as the replication cannot take place without the presence of a topological enzyme in solution. As soon as some molecules of topo II are added to the system*, eqs. (C.4)(C.6) quickly drive the system away from from this state and toward full replication, i.e. $\rho_{u}+\rho_{l}=2$, which is the true stable fixed point of the system.

This can be easily found by setting the left hand side of eqs. (C.4)-(C.6) to zero and by assuming that, thanks to diffusion, at the end of the replication time $T_{R}$ the densities of linked and unlinked mini-circles and of topo II are uniform across the Kinetoplast, i.e. $\rho_{u, l}\left(\boldsymbol{x}, T_{R}\right)=\rho_{u, l}\left(T_{R}\right)$ and $\phi_{T}\left(\boldsymbol{x}, T_{R}\right)=\phi_{T}\left(T_{R}\right)$, one obtains

$$
\rho_{l}^{*}=\frac{4}{R / A+2}=2-\rho_{u}^{*} .
$$

This shows that the final state of replication $S\left(t=T_{R}\right)$ corresponds to a system in which $\rho_{u}\left(\tau_{R}\right)+\rho_{l}\left(\tau_{R}\right)=\rho_{u}^{*}+\rho_{l}^{*}=2$ and that the relative abundance of linked and un-linked mini-circles

$$
\frac{\rho_{u}^{*}}{\rho_{l}^{*}}=\frac{1}{2} \frac{R}{A}
$$

is directly dependent on the ratio $R / A \equiv \beta$. In the case $\beta \simeq 0$ one can expand the stable fixed point in eq. C.8 and obtain

$$
\rho_{l}^{*} \sim 2-\beta+\mathcal{O}\left(\beta^{2}\right)
$$

and

$$
\rho_{u}^{*} \sim \beta+\mathcal{O}\left(\beta^{2}\right)
$$

In this case the final state corresponds to a system in which a small fraction $\rho_{u}^{*}$ of mini-circles is un-linked from the network and almost all the other mini-circles in the system belong to the Kinetoplast network.

Because the aim is to generate a fully linked network, one can naïvely imagine to simply set $R=0$ so that $\beta=0$; by doing so one instead forbids the network replication, as the initial removal is of paramount importance for the mini-circles to fully duplicate in the anti-podal complexes. The other option to minimise $\beta$ is to have a large attachment rate, i.e. the time taken by topo II to catalyse a singlestrand passage $\tau_{\text {cross }}$ has to be short. On the other hand, $\tau_{\text {cross }}$ should be itself related to the inverse of the activity (or the "turnover number") $k_{\text {cat }}$ of topo II Duplantier et al., 1995, which has been studied in the past by measuring the ATPase activity of DNA gyrase in presence of DNA substrates [Maxwell and Gellert, 1984]. In light of this, it is possible to relate the attachment rate $A$ to the experimentally measured turnover (ATPase activity) number as

$$
A \sim k_{\text {cat }} \sim 1 s^{-1}
$$

${ }^{*}$ This could be argued to be too sensitive to the presence of topo II, and some threshold could be added to regulate the sensitivity of $S(t=0)$ to the value $\phi_{T}$. In order to keep things simple I decided to neglect this correction. 
which provides a bound on the values assumed by this parameter. In other words, the value of the attachment rate $A$ cannot be arbitrarily changed, but depends on the bio-chemical structure of topo II and its efficiency. In addition, it has to be stressed that the removal of a mini-circle from the network requires, at least, three strand-crossing operations in order to free the ring from the average number of neighbours (which is three, see Ref. Chen et al., 1995b and Ch. 5). This implies that $R$ is necessarily smaller $A$, and taking into account the condition $R>0$, one arrives at

$$
0<\beta<1 .
$$

The expression in eq. (C.13) is a strong statement on the topology of the network. In fact, any state in which $R / A \equiv \beta$ is strictly greater than zero corresponds to a marginally linked network, i.e. a network that does not include all the mini-circles in the system. In particular, eqs. (C.8) and (C.13) imply $2 / 3<\rho_{l}^{*} /\left(\rho_{l}^{*}+\rho_{u}^{*}\right)<1$ and $0<\rho_{u}^{*} /\left(\rho_{l}^{*}+\rho_{u}^{*}\right)<1 / 3$ or (using eq. (C.9))

$$
0<\rho_{u}^{*} / \rho_{l}^{*}<1 / 2 .
$$

This means that in any case (even in the very unlikely case that $R=A$ ) the final fraction of mini-circles linked to the Kinetoplast core is the largest majority, i.e. the Kinetoplast will always form a unique spanning component. This is rather encouraging as this implies that the Kinetoplast will never fail to form a percolating core and it is therefore, in this respect, foolproof. Obviously, the best result is achieved at small $\beta$, for which the final state is the closest to a fully linked network. On the other hand, eqs. (C.12) and (C.13) imply that $\beta$ can be tuned only by acting on $R$ in the range $0<R<A$.

At this stage it is also worth pointing out that acting on $R$ is likely to not only affect the final topology of the network but also the time required to replicate the network in full $\left(T_{R}\right)$. By looking at eqs. (C.5) and (C.6) one can in fact notice that unlinked mini-circles are produced at rate $R$ from the linked structure proportionally to the local concentration of topo II, $\phi_{T}$, which is itself (indirectly) produced by the presence of unlinked mini-circles. In other words, decreasing $R$ is likely to have a double negative feedback effect on the network replication. It is therefore likely that $T_{R}$ is strongly dependent on $\beta$, and in particular, small removal rates $R$ might lead to very long replication times $T_{R}$.

Being the evolutionary pressure very high for these organisms, the replication time $T_{R}$ is a crucial parameter for their survival. Having a long replication time is in fact far from being an evolutionary advantage. On the other hand, their survival is also strongly related to their ability to pass on their vital genetic information, i.e. their ability to preserve the genetic material, which is itself strongly dependent on the linkedness of the network (Ch. 5). In light of this it is therefore tempting to speculate that the value of $\beta$ had been tuned by evolution to balance the need of a well-linked network, i.e. $\rho_{l}^{*} \simeq 2$, and that of a reasonably short time to complete replication.

The competition between these two effects can be summarised in one equation that describes the growth of a population of $N$ organisms as a function of two parameters: $\beta$ and $\gamma$. The latter can be interpreted as the critical lethal fraction of mini-circles unlinked from the Kinetoplast at the end of replication, beyond which 
the progeny cells are no longer viable. The equation reads:

$$
\frac{1}{N} \frac{d N}{d t}=T_{R}^{-1}(\beta)\left[1-\gamma\left(2-\rho_{l}^{*}(\beta)\right)\right]=f(\beta) .
$$

Eq. C.15 simply states that a population of $N$ organisms grows at a rate proportional to the speed of replication ${ }^{\dagger}$ i.e. proportionally to $T_{r}^{-1}(\beta)$ and to the likeliness of possessing a number $\rho_{u}^{*}=2-\rho_{l}^{*}$ of un-linked mini-circles smaller the critical lethal fraction $\gamma$. In other words, if $\beta$ and $\gamma$ are such that

$$
\gamma \rho_{u}^{*}(\beta)=\gamma\left(2-\rho_{l}^{*}(\beta)\right) \geq 1,
$$

the population cannot grow, as the Kinetoplasts generated are not retaining enough genetic material to ensure the viability of the progeny.

All this strongly relies on the crucial assumption that mini-circles which are not linked to the Kinetoplast at the end of the replication are most likely lost during cell division and are, therefore, not passed onto the progeny cells. This means that $\gamma$ sets a necessary fraction of mini-circles that need to be linked below which not enough genetic material is retained for the progeny to be viable.

Since $f$ measures the total growth rate of a population of Kinetoplasts, it gives a measure of the species evolutionary fitness. Maximising such fitness means finding the most "fit" pair $\{\beta, \gamma\}$. By setting $d f / d \beta=0$, one finds:

$$
\frac{d f}{d \beta}=-\frac{1}{T_{R}^{2}(\beta)} \frac{d T_{R}(\beta)}{d \beta}\left(1-\gamma \rho_{u}^{*}(\beta)\right)-\frac{1}{T_{R}(\beta)} \gamma \frac{d \rho_{u}^{*}}{d \beta}=0 .
$$

As I showed before, in the limit $\beta \ll 1$ one can approximate $\rho_{u}^{*} \sim \beta+\mathcal{O}\left(\beta^{2}\right)$, which gives:

$$
-\frac{1}{T_{R}^{2}(\beta)} \frac{d T_{R}(\beta)}{d \beta}(1-\gamma \beta)-\frac{1}{T_{R}(\beta)} \gamma\left(1-\frac{\beta}{4}\right)+\mathcal{O}\left(\beta^{2}\right)=0
$$

By assuming that in the range $0<\beta<1$ the replication time $T_{R}(\beta)$ cannot be zero or infinitely large, one can write:

$$
\frac{1}{T_{R}(\beta)} \frac{d T_{R}(\beta)}{d \beta}=-\gamma \frac{1-\beta / 4}{1-\gamma \beta}
$$

which, once again approximating for small $\beta$, leads to

$$
\frac{d}{d \beta} \log T_{R}(\beta) \simeq-\gamma[1+\beta(\gamma-1 / 4)] .
$$

This implies that in the lowest approximation, the functional form of the replication time $T_{R}(\beta)$ can be estimated as

$$
T_{R}(\beta) \sim e^{-\gamma \beta+\mathcal{O}\left(\beta^{2}\right)} .
$$

Although this functional form of the replication time should be, in principle, be recovered from eqs. (C.4)-(C.6), it is rather appealing (or fortunate) that it is obtained from maximising the fitness function in eq. C.15). On the other hand, this

\footnotetext{
${ }^{\dagger}$ In a time $T, N_{0}$ initial cells duplicate $T / T_{R}=m$ times, i.e. $N_{m}=N_{0} \exp (m \log (2))$.
} 


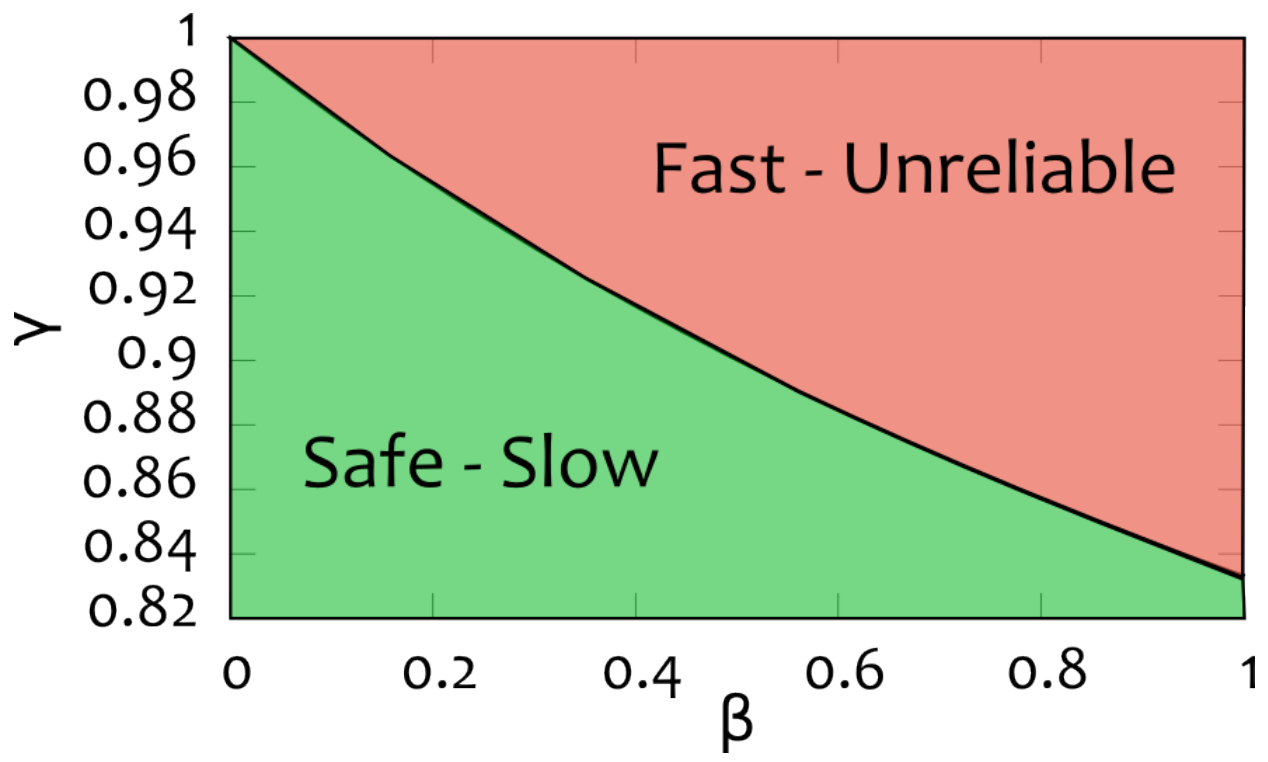

Figure C.2: Relation between the parameters $\gamma$ and $\beta=R / A$ as obtained in eq. C.23. For a given value of $\gamma$, eq. C.23 gives a threshold for $\beta^{*}$ above which the replicated network (although built faster) is too poorly linked to retain the necessary fraction of genetic material $\gamma$, and the progeny is therefore not viable. On the other hand below $\beta^{*}$ the replicated network is safely generated as the linkedness of the network ensures that enough genetic material is passed on, although $T_{R}^{-1}$ is much slower. The balance is given by the thick line for which the replication rate is the fastest given that a fraction $\gamma$ of genetic material needs to be linked to the Kinetoplast and it identifies, therefore, the "maximum fitness" line.

restricts the validity of eq. C.21) to the case in which the fitness is maximum, i.e. a precise combination of $\gamma$ and $\beta$, rather than to the whole space and also to the case in which $\beta$ is small. It is worth noticing that this functional form is also supported by numerical evidence Michieletto et al., 2014. In light of this, one can assume that $T_{R}$ takes the form in eq. (C.21) and go back to eq. (C.17) to write

$$
\gamma e^{\gamma \beta}\left(\frac{\beta+2-2 \gamma \beta}{\beta+2}\right)-\gamma e^{\gamma \beta} \frac{4}{(\beta+2)^{2}}=0
$$

and finally obtain

$$
\gamma=\frac{1}{2} \frac{\beta+4}{\beta+2} .
$$

In Fig. C.2 I show the curve given by eq. C.23 which identifies the maximum fitness line in the space $(\gamma, \beta)$. As mentioned before, $\gamma$ describes the critical lethal fraction of mini-circles unlinked from the Kinetoplast at the end of the replication, i.e. $\rho_{u}^{*}=2-\rho_{l}^{*}$. Because each mini-circle carries genetic material, the parameter $\gamma$ can also be interpreted as "redundancy" in the genetic material carried by the mini-circles. It is worth noting that in the limit $\gamma \rightarrow 1$, the system requires $\beta \rightarrow 0$ in order to satisfy eq. C.23. This means that every mini-circle needs to be linked to the network at the end of the replication, i.e. every mini-circle is crucial for the survival of the organism (see eq. (C.15)). This can be interpreted with the fact that, in this case, each mini-circle carries unique genetic information, and any loss of mini-circles would cause the reduction of evolutionary fitness. In other words, setting the parameter $\gamma=1$, signifies that there is no redundancy in the genetic material carried by the mini-circles, and each one of them has to be passed on to 
the progeny in order for the organism to survive.

On the other hand, the mini-circles in the Kinetoplast have been observed to possess a slightly redundant genetic information, meaning that they encode largely heterogeneous genetic material but display some highly conserved genes Jensen and Englund, 2012. This is in agreement with eq. (C.23) and Fig. C.2 and, in particular, sets a rough estimation of $\gamma$ below unity. This implies that $\beta$ is not so close to zero and therefore the final network is most likely to be marginally linked and the replication process to be faster than it would be if $\gamma$ was set to one.

All this also implies that the initial assumption that $\beta \sim 0$ is not crude at all, in particular because (i) the Kinetoplast is notably well linked, i.e. $\rho_{u}^{*} \simeq 0$ and $\rho_{l}^{*} \simeq 2$ and (ii) the redundancy of the genetic material is strictly greater than zero, although very small, i.e. $\gamma \simeq 1$.

It is important to bear in mind that all this is dependent on the assumption that any mini-circle which is not linked to the Kinetoplast at the end of the replication is lost during the separation into progeny cells. While this assumption might at first sound too crude, it is worth stressing that it is supported by experimental evidence, which assessed the viability of the progeny cells when topo II is inhibited from the Kinetoplast, i.e. $\kappa$ in eq. (C.6) set to zero. This means that the Kinetoplasts were not able to topologically regulate their formation, and as a consequence, the resulting networks appeared fragmented, not forming a spanning component and ultimately led to progeny cells death Wang et al., 2000, Wang and Englund, 2001. All this implies that the formation of a nearly perfect spanning component is crucial for the organism survival and this could have been evolutionarily adapted, by introducing some genetic redundancy, to allow a faster replication.

\section{Conclusions}

In brief, in this Appendix I introduced an analytical model for the replication of the Kinetoplast DNA. Although this model aggressively simplifies this complex biological system, some of its crucial assumptions are supported by experimental evidence and a careful analysis of the results can give some insight into the Kinetoplast unique structure.

In particular, the replication process can be described as a self-regulated process, requiring only the input of a small initial level of topoisomerase to fully replicate the Kinetoplast. The final topological state of the network is found to be crucially controlled by the ratio $\beta=R / A$, with the degree of linkage decreasing with increasing $\beta$. I also showed that taking into account precision and speed of the replication, it is possible to explicitly write a fitness function whose maximisation led to a maximum fitness line in the parameter space $(\gamma, \beta), \gamma$ being a "redundancy factor" or the critical fraction of unlinked mini-circles at the end of the replication

Finally, a qualitative comparison between the findings presented here and the material available in the literature (Ref. [Jensen and Englund, 2012] and therein), encourages the speculation that the organisms of the class Kinetoplastida tuned the ratio $\beta$ in order to find a balance between speed and accuracy of replication, thereby possessing a marginally linked Kinetoplast DNA structure with some redundancy in the genetic material. Based on the findings presented here, it is my hope that further biological experiments on this structure will shed new light onto the complicated and beautiful Kinetoplast. 


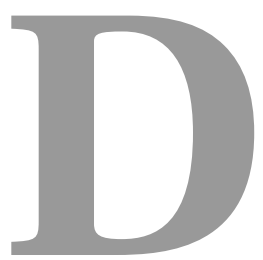

\section{Non-equilibrium form of Differential Mobility}

Here, the formula for the non-equilibrium mobility $\mu_{D}^{A}$ is derived for one degree of freedom $z(t)$. For simplicity, I consider over-damped dynamics, an approximation that applies well to the case studied in Sec. 6.1. The case of underdamped Langevin dynamics was also discussed in a previous work Baiesi et al., 2011]. The overdamped equation of motion of a bead driven by the force $\Psi(z(t))$ and which is perturbed by the addition of a potential $-V(z) h(t)$ (where $h$ is a small parameter) and with inverse friction coefficient $\nu=1 / \zeta$ at temperature $T\left(k_{B}=1\right)$ is

$$
\frac{d z(t)}{d t}=\nu\left[\Psi(z(t))+h(t) \frac{\partial V}{\partial z}\right]+\sqrt{2 \nu T} \tilde{\eta}(t)
$$

where $\tilde{\eta}(t)$ is a standard white noise. Under quite general conditions, the response function for the observable $O(t)$ in overdamped systems Baiesi et al., 2009, Baiesi et al., 2011 is

$$
R_{O V}(t, s)=\left.\frac{\delta\langle O(t)\rangle^{h}}{\delta h(s)}\right|_{h=0}=\frac{1}{2 T}\left[\frac{d}{d s}\langle V(s) O(t)\rangle-\langle L V(s) O(t)\rangle\right],
$$

where $L$ is the backward generator of the Markov dynamics,

$$
L=\nu \Psi(z) \frac{\partial}{\partial z}+\nu T \frac{\partial^{2}}{\partial z^{2}}
$$

in this case. The susceptibility

$$
\chi_{O V}(t)=\int_{0}^{t} R_{O V}(t, s) d s=\frac{1}{2 T}\left[\langle[V(t)-V(0)] O(t)\rangle-\left\langle\int_{0}^{t} L V(s) d s O(t)\right\rangle\right]
$$

represents the linear response to a constant perturbation turned on at time $t=0$. In this case, the mobility is the susceptibility of the average velocity to the addition of a constant force, i.e. the observable is $O(t)=\Delta z(t) / t$ and the perturbation is 
$V(z)=z$, leading to $L V(z)=\nu \Psi(z)$. Hence (D.4) simplifies to

$$
\mu_{D}^{A}(t)=\frac{1}{2 T}\left[\left\langle\Delta z(t) \frac{\Delta z(t)}{t}\right\rangle-\left\langle\int_{0}^{t} \nu \Psi(s) d s \frac{\Delta z(t)}{t}\right\rangle\right] .
$$

Since the system is out of equilibrium, the average displacement $\langle\Delta z(t)\rangle$ is not zero. It is appropriate to remove it from the terms in eq. (D.5) and using $\langle\Delta z(t)\rangle=$ $\left\langle\int_{0}^{t} \nu \Psi(s) d s\right\rangle$ one gets

$$
\mu_{D}^{A}(t)=\frac{1}{T}\left[\frac{\langle\Delta z(t) ; \Delta z(t)\rangle}{2 t}-\frac{1}{2 t}\left\langle\int_{0}^{t} \nu \Psi(s) d s ; \Delta z(t)\right\rangle\right]
$$

where $\langle a ; b\rangle \equiv\langle a b\rangle-\langle a\rangle\langle b\rangle$ denotes the covariance. The first term estimates the spread around the average position and can thus be interpreted as a diffusion constant, while the second represents a novel nonequilibrium term. The formula remains unaltered if the force $\Psi$ depends on many degrees of freedom, as long as their noises are statistically independent. The expression in eq. (D.6) is the one we used to compute the differential nonequilibrium mobility $\mu_{D}^{A}$ in Sec. 6.1.2 eq. 6.5), letting $t$ become sufficiently large. Since beads are equivalent, I have also averaged the mobility of all beads. 


\section{$\mathrm{E}$ \\ Gel Electrophoresis of DNA Knots}

\section{Trajectories of DNA knots}

One can compute the mobility of knotted polymers by measuring the centre of mass displacement along the direction of the applied force In Fig. E.1 I show examples of trajectories of the knots and their relative change of radius of gyration during in silico gel electrophoresis experiments. In Fig. E.1. I also compare trajectories and speed of knots travelling in regular and irregular gels. As one can notice, while in irregular gels (Fig. E.1(a)-(b)) the motion of the knots under weak (a: $50 \mathrm{~V} / \mathrm{cm}$ ) and moderate (b: $150 \mathrm{~V} / \mathrm{cm}$ ) fields displays a rather different behaviour, in regular gels (Fig. E.1(c)-(d)) knots that are driven by the same external fields (c: $50 \mathrm{~V} / \mathrm{cm}$ and d: $150 \mathrm{~V} / \mathrm{cm}$ ) but display similar trajectories. In particular, a field inversion is never observed at any field or gel density (Fig. E.1(e)-(g)).

\section{The role of Hydrodynamics}

The Brownian Dynamics scheme allows us to introduce an implicit solvent, i.e. there is no explicit hydrodynamics taken into account during the simulations. I made this choice assuming that in the case of dense gels, the contribution coming from hydrodynamics would be negligible compared to the interactions of the polymers with the environment. This is confirmed in Fig. E.1 where I show the linearly increasing speed of knots travelling through a regular gel at weak and strong fields. In both cases, even without the presence of hydrodynamics or rescaling, it is possible to recover the expected linear relationship between speed and ACN. In Weber et al., 2006b the authors rescaled the final velocities of the knots by using the Kirkwood-Risenman formula, thereby accounting for hydrodynamics. In this work I observed that such rescaling does not affect the final functional form of the results, consequently I did not introduce such rescaling. I argue that the contribution from hydrodynamics becomes relevant when sparser gels are considered. In this case one can notice (data not shown) that the rescaling operated in Weber et al., 2006b] is necessary to recover the experimental observations as no electrophoretic separation is observed (see Fig. E.1(f)). 

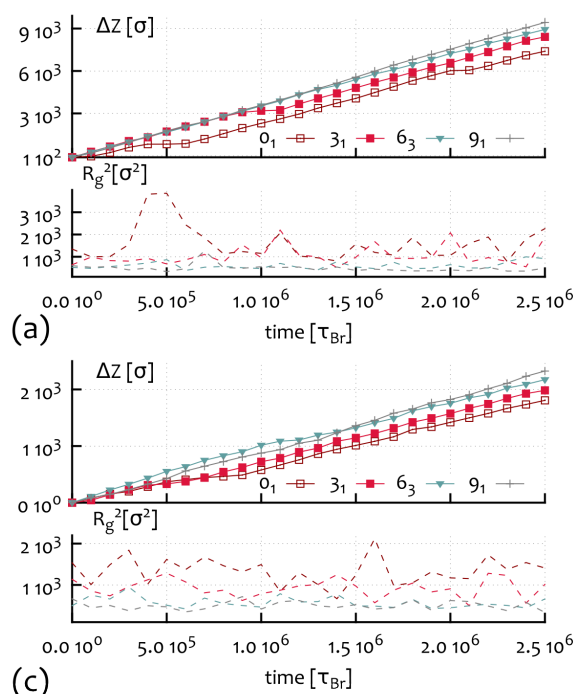

(b)
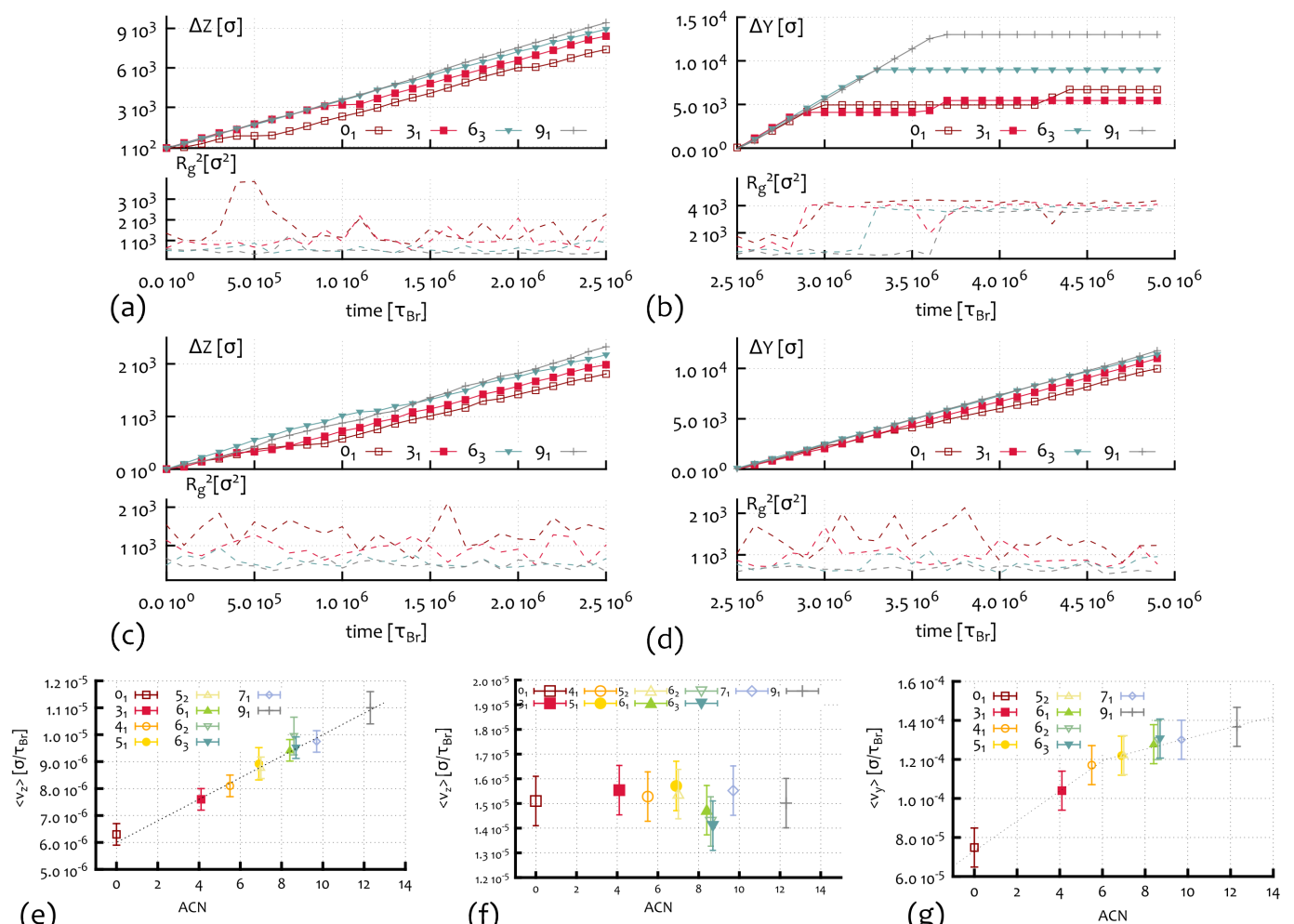

Figure E.1: Panels (a) and (b): trajectory and squared radius of gyration vs time for some knotted DNA loops subject to weak $(50 \mathrm{~V} / \mathrm{cm})$ (a) and moderate $(150 \mathrm{~V} / \mathrm{cm})$ (b) electric fields and moving through an irregular gel, i.e. with dangling ends. Panels (c) and (d) refer to the regular gel case with same lattice spacing. Note that for high fields and in gel with dangling ends the knotted loops are trapped (zero velocity) for long time in states with large radius of gyration, suggesting an entangled or "impalement" state, while in a regular gel the difference between the trajectories at weak and moderate fields is very little. (e) The velocity of knotted polymers in regular gels, i.e. without dangling ends, displays a monotonic behaviour as a function of the average crossing number. For dense enough gels $(l=200 \mathrm{~nm})$, the contribution coming from the interaction with the structure is observed to be sufficient to recover the linear electrophoretic separation as a function of the average crossing number at weak fields $(E=1.25 \mathrm{~V} / \mathrm{cm})$. This is instead not observed in the case of sparser gels $(l=500 \mathrm{~nm})$, where one recovers no electrophoretic separation (f). It is possible to observe a deviation from the linear trend only at stronger fields and denser gels $(E \gtrsim 12.5 \mathrm{~V} / \mathrm{cm}, l=200 \mathrm{~nm})$, although I never observed a field-inversion or non-monotonic behaviour (g).

\section{Waiting Times}

In order to quantify the time taken by the rings to disentangle from the impalements, I analysed the single trajectories (reported for example in Fig. E.1) and computed the amount of time each knot moves slower than it would if it was free, i.e. $v_{\text {free }}=$ $f / \zeta$. From this one can quantify the total amount of time each knot is stuck in an entangled state. This is found to be a non-monotonous function of the ACN (see Fig. E.2(h)) and is in line with the non-monotonic average speed of the knots and the relative separation in gel electrophoresis experiments as a function of their ACN.

Each time a knot is stalled, it takes a certain amount of time to re-establish its motion. We report the distribution of these "waiting times" $\left(\tau_{\text {dis }}\right)$ in Fig. E.2(a) $-(\mathrm{g})$ for several knots. As one can notice, the distribution is bimodal, i.e. shows two 

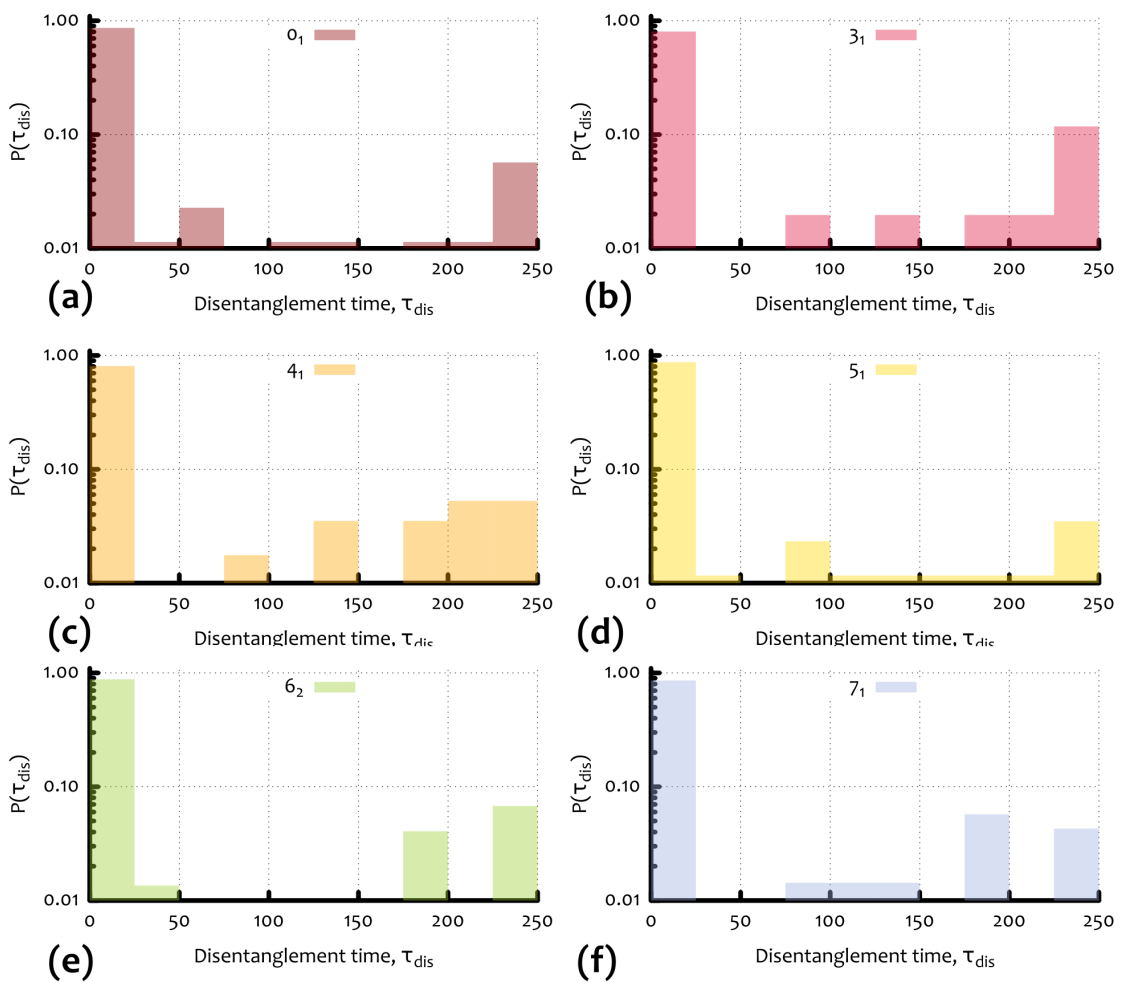

(e)

(f)
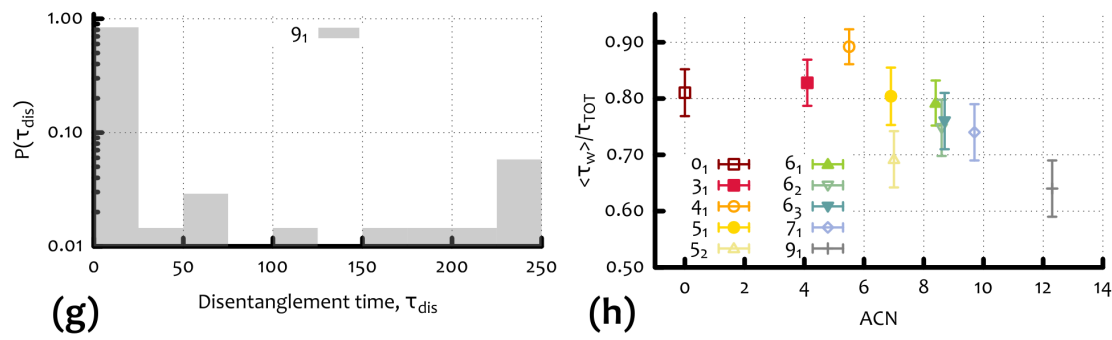

Figure E.2: (a)-(g) Waiting time distribution $P\left(\tau_{\text {dis }}\right)$ for different knot types and in units of simulation Brownian time $\tau_{B r}$. One can notice that the distribution shows to peaks at short and large times which correspond to "transverse" or "head on" interactions, respectively. (h) Total amount of time spent in an entangled state as a function of the $\mathrm{ACN}$. This quantity reflects the fact that slower knots spend more time in entangled states (compare with Fig. 6.7 in Sec. 6.2.

distinct peaks at short and long times. We interpret the peak at short time-scales as transversal impalements or crawling around the gel structure, while the peak at long times as the fact that sometimes knots interact via an "head-on" collision with the gel open strands. This immobilises the knots for long time, as the only way to re-establish the motion is by moving against the field for a length of at least half lattice spacing.

\section{Biased Continuous-Time Random Walk Model}

Here I give the details of the biased continuous time random walk (CTRW) model, whose results are reported in Sec. 6.2 .

One starts by noticing that the simulations suggest the existence of two important time-scales in the dynamics: $\tau_{f}$ and $\tau_{\text {dis }}$, respectively regulating the frequency 
of entangling events and the time required to disentangle from the impalements with the dangling ends. Firstly, one can address the situation in which $\tau_{\text {dis }} \ll 1$. In this case the knots disentangle quickly, and one can think of the whole process as random walks biased in the opposite (the DNA is negatively charge) direction of the field. Due to topological interactions with the gel, the biased random walkers eventually stops with a rate $\lambda_{e} \simeq \tau_{f}^{-1}$. This rate is expected to be described by the probability $P_{e}(r / l)$ of a circle of radius $r$ to overlap onto an edge of a $2 \mathrm{D}$ square mesh with lattice spacing $l$, i.e.

$$
\lambda_{e}(\mathcal{K}) \simeq \frac{P_{e}(x(\mathcal{K}))}{P_{e}\left(x\left(0_{1}\right)\right)} \lambda_{e}\left(0_{1}\right),
$$

where $x(\mathcal{K})=R_{g}(\mathcal{K}) / l$. Note that this rate is normalised with respect to the empirical value observed in the Brownian dynamics simulations for the unknot $\left(0_{1}\right)$ at weak fields.

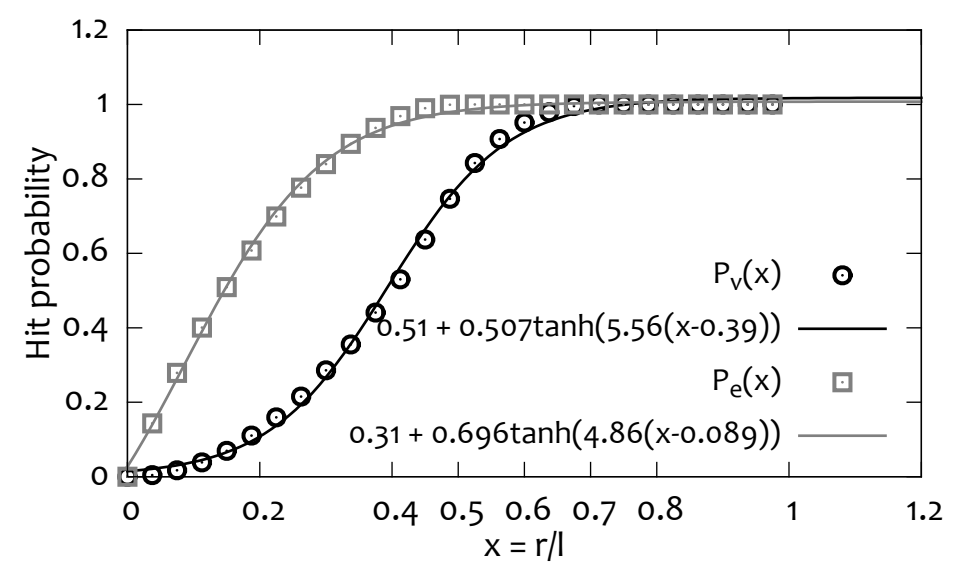

Figure E.3: Probability for a circle of radius $r$ drawn randomly on a square lattice with lattice spacing $l$ to encircle a lattice vertex $\left(P_{v}\right.$ - black circles) and to overlap over a lattice edge ( $P_{e}$ - gray squares). The two quantities reach 1 for $r=l / 2\left(P_{e}\right)$ and for $r=l / \sqrt{2} \simeq 0.7$ $\left(P_{v}\right)$. The functions and parameters used to fit the data are also shown.

One can obtain the precise value of $P_{e}(x)$ as a function of the ratio $R_{g}(\mathcal{K}) / l$, with $l$ the gel lattice spacing from Fig. E.3, where I simulated the deposition of a circle of radius $r$ onto a 2D mesh with lattice spacing $l$ and calculated the probability of overlapping over a strand $\left(P_{e}(x)\right)$ or to encircle a vertex $\left(P_{v}(x)\right)$ of the grid.

The knots moving in the gel are therefore viewed as random walkers on a lattice. They can jump one site to their right (broadly this corresponds to one unit cell of the gel), or are required to stop for one time-step (because $\tau_{\text {dis }} \ll 1$ ) with rate $\lambda_{e}$. This is equivalent to a Poisson process whose probability of getting entangled after $t$ time-steps is given by:

$$
P_{\text {ent }}(x(A C N), t)=1-\left[1-\lambda_{e}\left(R_{g}(\mathcal{K})\right)\right]^{t} \simeq 1-\exp \left[-\lambda_{e}(\mathcal{K}) t\right]
$$

where we stress the dependence of $\lambda_{e}$ on the knot type via its size $R_{g}$. The average time between interactions is given by $\left\langle\tau_{f}\right\rangle \simeq \lambda_{e}^{-1}$ and the average number of stops on a time $t$ is $\left\langle n_{e}\right\rangle=\lambda_{e} t$. The relative separation between two knots can be expressed 
as

$$
\begin{aligned}
\Delta x\left(\mathcal{K}_{1}, \mathcal{K}_{2}\right) & =v_{\text {free }}\left(t_{\text {free }}\left(\mathcal{K}_{1}\right)-t_{\text {free }}\left(\mathcal{K}_{2}\right)\right) \\
& =v_{\text {free }} t\left(\lambda_{e}\left(\mathcal{K}_{2}\right)-\lambda_{e}\left(\mathcal{K}_{1}\right)\right)
\end{aligned}
$$

where $v_{\text {free }}$ is the velocity of the knots when no interactions occur (when hydrodynamics is negligible this is the same for all knots), $t$ is the observation time and $t_{\text {free }}=t-\lambda_{e} t$. From this is clear that the separation between knots $\mathcal{K}_{2}$ and $\mathcal{K}_{1}$ is proportional to the difference of their entanglement rates, as expected. Given that our model relies on the hitting probability between a knot and its environment, it is clear that if the knot size is either much larger or much smaller than the gel lattice spacing, the electrophoretic separation is not as good as in the case the two are comparable. Within our simple model we recover good linear relationship within the range $R_{g} / l \in\left[10^{-4}, 10\right]$ meaning that short samples (or large gel pores compared to the gyration radius of the knots) work better for the electrophoretic separation at weak fields rather than small pores (or very large $R_{g}$ ) Dorfman, 2010 (see Fig. E.4(a)). This is compatible with the fact that is often impractical to perform gel electrophoresis with very large samples. In fact, for other cases outside this range, I observe essentially no spatial separation of the knots (see Fig. E.4(a)).

Now, I will introduce a topology dependent disentanglement time: every time that the Random Walker undergoes a "stop", I require that it has to wait an amount of time that increases proportionally to its average entanglement number (see $\langle\pi\rangle$ in Fig. 6.8). The disentanglement time is picked from an exponential distribution with characteristic time given by the empirical one observed in the MD simulations (see Fig. 6.9), i.e.

$$
t_{w}=-\ln x / \lambda_{\text {dis }}
$$

where $x$ is a random number between 0 and 1 and $\lambda_{\text {dis }}^{-1}=\tau_{\text {dis. }}$. As observed in the main text, the disentanglement time $\tau_{\text {dis }}$ is expected to be a function of $\langle\pi\rangle$, i.e. to increase linearly with the ACN. One can therefore use:

$$
\tau_{\text {dis }}(A C N)=A\langle\pi\rangle+\tau_{\text {dis }}\left(0_{1}\right)
$$

where $A=5 \tau_{B r}$ is an empirical parameter fitted from the simulations and $\tau_{d i s}\left(0_{1}\right)=$ $30 \tau_{B r}$ is the empirical average disentanglement time of the unknot. In addition, long waiting times which are typical of head-on impalements are taken into account by adding a small probability $(q \simeq 0.05$, compatible with the bimodal distribution in Fig. E.2 and conditioned to the fact that an entanglement event happened. For this one can set $\tau_{\text {long }}=A\langle\pi\rangle+250$. So the average disentanglement time from a headon impalement is still knot-dependent but is much longer than any characteristic disentanglement time for other types of entanglements.

Within this model one can recover the electrophoretic arc as shown in Fig. E.4(b). In addition, the shape of the arc can be finely tuned to obtain different depths of the arc and different slowest knots. This is mainly controlled via the ratio $R_{g} / l$ (where it is implicitly assumed that the external field is neither too weak or too strong). In Fig. 6.10 of the main text I show that within this model one can accurately recover the electrophoretic speed of the knots observed in the MD simulations by shifting the curve obtained with the CTRW model setting $R_{g}\left(0_{1}\right) / l=0.5$ (as in the MD simulation) to the observed speed of the unknot and by rescaling it by the free speed $v_{\text {free }}$ at which the knots travel between two planes of the gel, i.e. two lattice 
sites in the CTRW model. It is worth noting also that this model allows us to predict the mobility of knots which were not probed by Brownian dynamics, by making use of the empirical functional forms of $\langle\pi\rangle$ and $R_{g}$ derived from these simulations. It is also worth stressing that the only real free parameter of this model is the lattice spacing $l$, while the value of $R_{g}\left(0_{1}\right), \tau_{\text {dis }}\left(0_{1}\right)$ and $\lambda_{e}\left(0_{1}\right)$ are input parameters needed to calibrate the model to a target case.
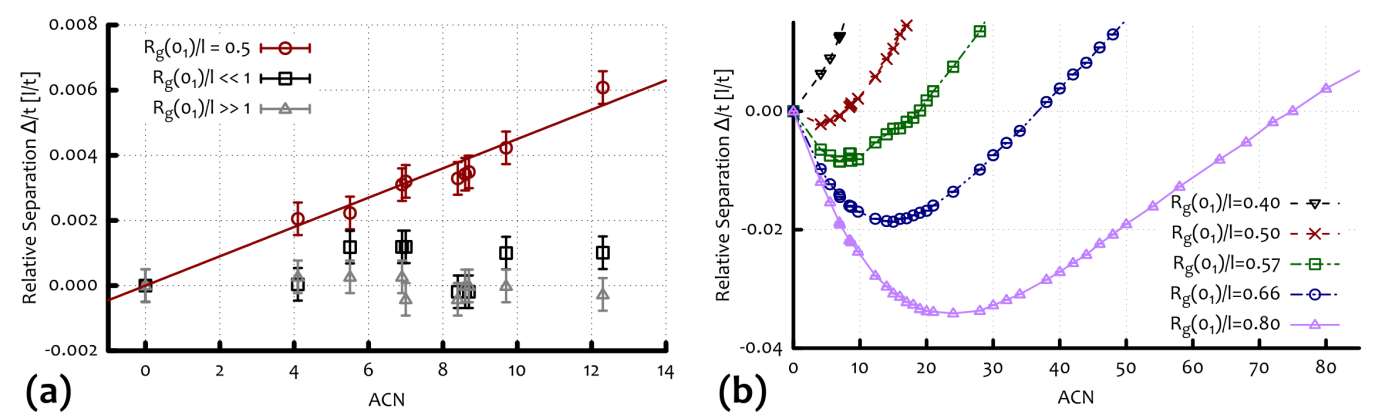

Figure E.4: Average relative separation over time (in units of lattice spacing over time) of the knots as a function of the ACN obtained from the simple CTRW model described in the text. (a) For weak fields (for which the disentanglement time is quick $\tau_{\text {dis }} \ll 1$ ) the linear relationship is re-established for $R_{g} \sim l$, while in the two limiting cases $R_{g} \gg l$ and $R_{g} \ll l$ we do not observe a clear separation, as expected. (b) The non-monotonic behaviour of the separation is re-established when a topology-dependent disentanglement time is introduced in the model. The typical electrophoretic arc crucially depends on the ratio $R_{g}(A C N) / l$. (See text for details). 


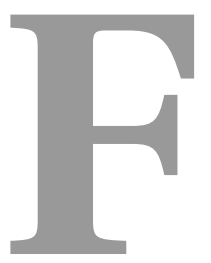

\section{Threading Identification Algorithm}

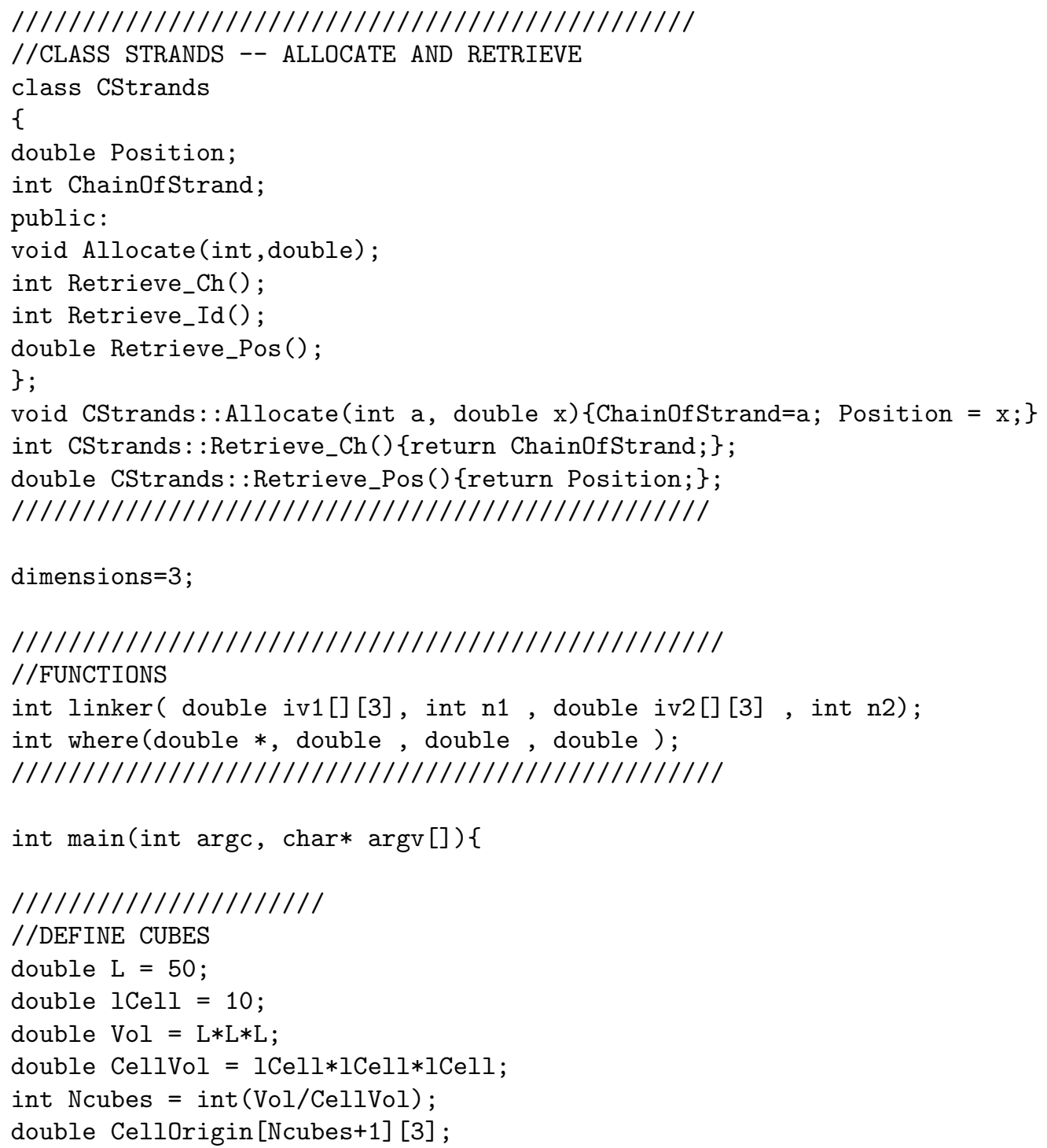




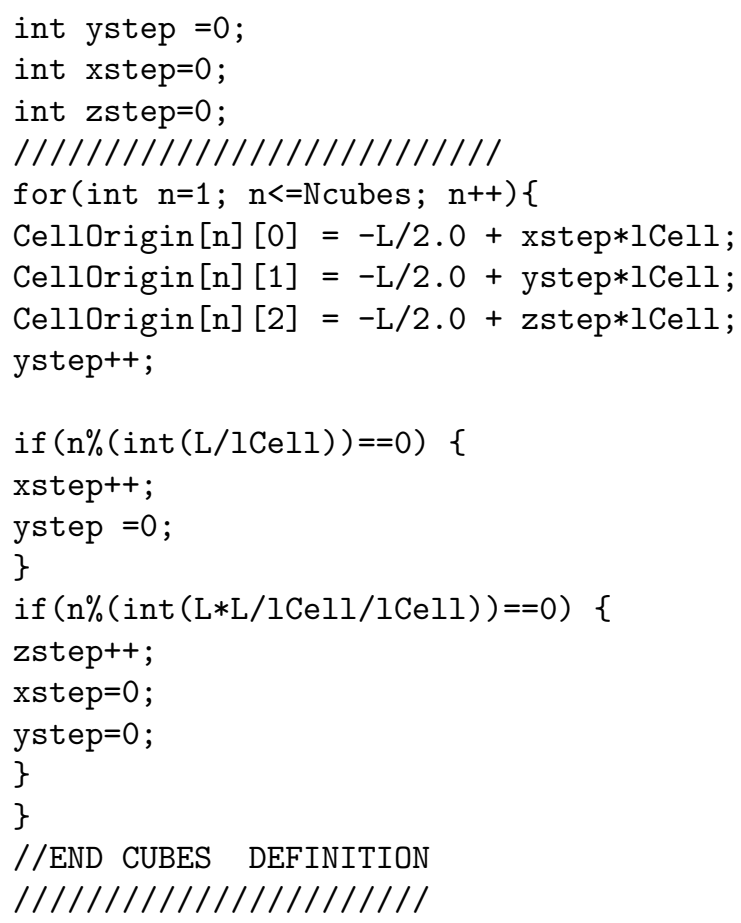




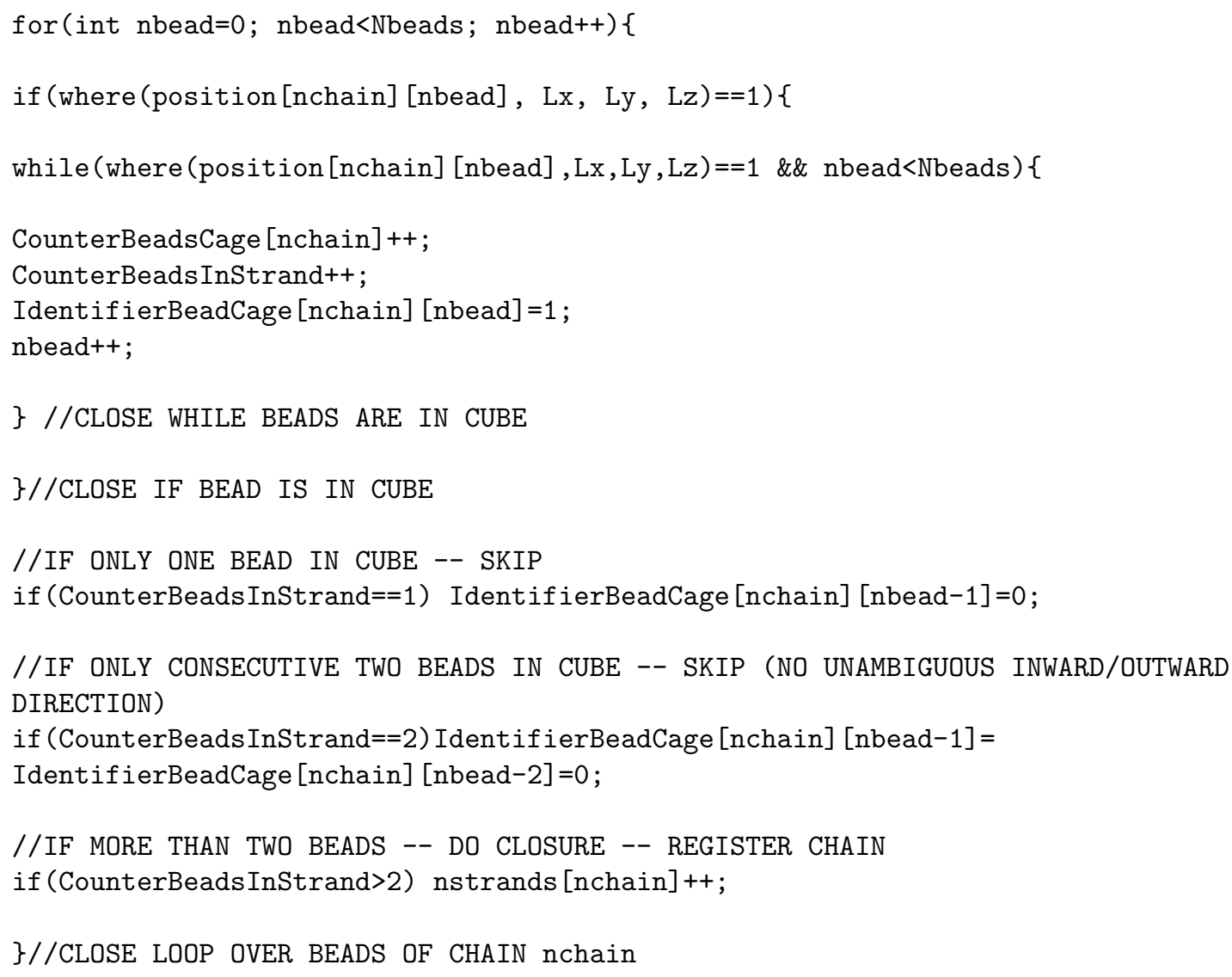

//WHILE THE BEADS OF THE STRANDS ARE INSIDE THE CUBE while (IdentifierBeadCage [nchain] [mp]==1 ) \{

//CREATE VIRTUAL STRANDS MADE OF THESE BEADS

PosStrands [indexstrands] [strandbeads] [0] . Allocate (nchain, position [nchain] [mp] [0]); PosStrands [indexstrands] [strandbeads] [1] . Allocate (nchain, position [nchain] [mp] [1]); PosStrands [indexstrands] [strandbeads] [2] . Allocate (nchain, position [nchain] [mp] [2]); strandbeads++;

$\mathrm{m}++$;

$\mathrm{mp}=\mathrm{m} \%$ Nbeads;

\}//CLOSE WHILE BEADS IN CUBE

//REGISTER SIZE OF VIRTUAL STRAND AND INDEX

SizeOfStrand [indexstrands] = strandbeads;

indexstrands++;

\}//CLOSE LOOP OVER STRANDS

nstrands [nchain] =indexstrands ; 
//CREATE VIRTUAL RINGS JOINING THE STRANDS IN CUBE//

//////////////////////////////////////////////

//LOOP OVER STRANDS VISITING CUBE

for (int ns=0; ns < indexstrands; nst+) \{

//LOOP OVER SIZE OF STRAND

for (int $i=0 ; i<$ SizeOfStrand [ns]; i++) \{

//ADD BEADS TO BIG RING CALLED "MassiveChain"

for $d$ in dimensions

MassiveChain [NumberOfMassive] [CounterBeadsMassive] [d] = PosStrands [ns] [d].Retrieve_Pos();

CounterBeadsMassive++;

\}

$1 / / / / / / / / / / / / / / / / / / / / / / / / / / / / / / / / / / / / / / /$

$/ /$ IF THE STRAND IS NOT THE LAST

//FILL GAP BETWEEN THIS AND SUCCESSIVE STRAND

//GAP IS THE VECTOR JOINING LAST BEAD OF THIS STRAND AND

//FIRST BEAD OF NEXT

if (ns<indexstrands-1) \{

for $d$ in dimensions

Gap [d] =-MassiveChain [NumberOfMassive] [CounterBeadsMassive-1] [d] +

PosStrands [ns+1] [0] [d] .Retrieve_Pos ( );

//FILL

Nfill=10; //use 10 bead to fill the space between consecutive strands

for (int $\mathrm{y}=0 ; \mathrm{y}<\mathrm{Nfill} ; \mathrm{y}^{++}$) \{

for $d$ in dimensions

MassiveChain [NumberOfMassive] [CounterBeadsMassive] [d] =

MassiveChain [NumberOfMassive] [CounterBeadsMassive-1] [d] + Gap [d]/(Nfill*1.0);

CounterBeadsMassive++;

\}//CLOSE LOOP OVER FILLING BEADS

\}//CLOSE IF "NOT LAST STRAND"

//IF LAST STRAND

//JOIN WITH *FIRST* STRAND

//IN ORDER TO FORM A RING

if ( $\mathrm{ns}==$ indexstrands-1) \{

for $d$ in dimensions

Gap [d] =-MassiveChain [NumberOfMassive] [CounterBeadsMassive-1] [d] +

PosStrands [0] [0] [d] .Retrieve_Pos ();

$/ /$ FILL

for (int $\mathrm{y}=0 ; \mathrm{y}<\mathrm{Nfill} ; \mathrm{y}++)\{$

for $d$ in dimensions

MassiveChain [NumberOfMassive] [CounterBeadsMassive] [d] =

MassiveChain [NumberOfMassive] [CounterBeadsMassive-1] [d] + Gap [d]/(Nfill*1.0);

CounterBeadsMassive++;

\}//CLOSE LOOP OVER FILL

\}//CLOSE IF "LAST STRAND"

\}//CLOSE ROUTINE TO GENERATE "MASSIVE"

//RINGS CONTAINED INSIDE THE CUBE ncube 
$1 / / / / / / / / / / / / / / / / / / / / / / / / / / / / / / / / / / / / / /$

$/ / / / / / / / / / / / / / / / / / / / / / / / / / / / / / / / / / / / / / /$

//START GENERATING EXTENDED STRANDS ///

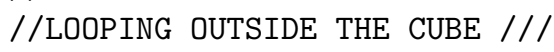

$/ / / / / / / / / / / / / / / / / / / / / / / / / / / / / / / / / / / / / / / / /$

//USE 50 BEADS TO EXTEND STRANDS

int $\mathrm{Fill}=50$;

//USE 100 BEADS TO FILL THE GAP BETWEEN EXTENDED STRANDS

int Ffill=100;

//LOOP OVER ALL THE STRANDS BELONGING TO CHAIN nchain

//VISITING THE CUBE

for (int $\mathrm{ns}=0$; $\mathrm{ns}<$ indexstrands; $\mathrm{ns}^{++}$) \{

CounterBeadsExtStr $=0$;

ChainOfExtStr [ns]=nchain;

//DEFINE TANGENT TO CURVES

//GOING IN THE CUBE

for $d$ in dimensions

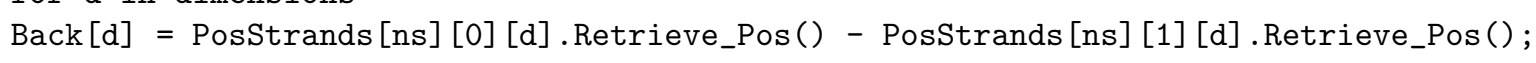

//GOING OUT THE CUBE

for $d$ in dimensions

Forward [d] = PosStrands [ns] [Size0fStrand [ns]-1] [d].Retrieve_Pos ()$-$

PosStrands [ns] [SizeOfStrand [ns]-2] [d] .Retrieve_Pos ();

//DEFINE THE VECTOR JOINING THE EXTENDED STRANDS

for $d$ in dimensions

Final $[d]=$

(-(PosStrands [ns] [SizeOfStrand[ns]-1] [d] .Retrieve_Pos ()+Forward [d]*Fill) +

(PosStrands [ns] [0] [d] .Retrieve_Pos ()$+$ Back [d] *Fill)) $/($ Ffill*1.0 + 1.0);

//GENERATE EXTENDED RINGS

//START FROM THE BEADS INSIDE THE CUBE

for (int $\mathrm{m}=0 ; \mathrm{m}<$ SizeOfStrand $[\mathrm{ns}] ; \mathrm{m}++$ ) \{

for $d$ in dimensions

ExtendedStrands [nom] [ns] [CounterBeadsExtStr] [d] = PosStrands [ns] [m] [d].Retrieve_Pos();

CounterBeadsExtStr++;

\}//CLOSE LOOP OVER BEADS IN STRAND

//EXTEND STRAND IN FORWARD DIRECTION

for (int $\mathrm{y}=0 ; \mathrm{y}<\mathrm{Fill} ; \mathrm{y}++)\{$

for $d$ in dimensions

ExtendedStrands [nom] [ns] [CounterBeadsExtStr] [d] =

ExtendedStrands [nom] [ns] [CounterBeadsExtStr-1] [d] + Forward [d];

CounterBeadsExtStr++;

\}//CLOSE EXTENSION

//FILL GAP TOWARD BACKWARD EXTENDED STRAND

for (int $\left.\mathrm{y}=0 ; \mathrm{y}<\mathrm{Ffill} ; \mathrm{y}^{++}\right)\{$

for $d$ in direction

ExtendedStrands [nom] [ns] [CounterBeadsExtStr] [d] =

ExtendedStrands [nom] [ns] [CounterBeadsExtStr-1] [d] + Final [d] ;

CounterBeadsExtStr++; 
\}//CLOSE GAP FILLING

//EXTEND (INVERTED) STRAND IN BACKWARD DIRECTION

for (int $\left.\mathrm{y}=0 ; \mathrm{y}<\mathrm{Fill} ; \mathrm{y}^{++}\right)\{$

for $d$ in dimensions

ExtendedStrands [nom] [ns] [CounterBeadsExtStr] [d] =

ExtendedStrands [nom] [ns] [CounterBeadsExtStr-1] [d] - Back[d];

CounterBeadsExtStr++;

\}//CLOSE INVERTED EXTENSION

//REGISTER SIZE OF NEW EXTENDED RING -- COMPLETED

SizeOfExtStr [nom] [ns] =CounterBeadsExtStr -1 ;

\}//CLOSE LOOP OVER ALL POSSIBLE STRANDS BELONGING TO CHAIN nchain

//REGISTER PROPERTIES OF NEW VIRTUAL MASSIVE CHAIN

//CONTAINED INSIDE CUBE

NumOfStrandsOfMassive[nom] = indexstrands;

ChainOfMassive[NumberOfMassive] = nchain;

SizeOfMassive[NumberOfMassive] = CounterBeadsMassive-1;

NumberOfMassive++;

////////////////////////////

\}//CLOSE LOOP OVER CHAINS

int NtotMassive $=$ NumberOfMassive;

$/ / / / / / / / / / / / / / / / / / / / / / / / / / / / / / / / / / / / / / / / / /$

//IN ORDER TO ENSURE CORRECTEDNESS OF CONSTRUCTION

//TEST LINKING NUMBER BETWEEN NEW VIRTUAL MASSIVE RINGS

for ( 1 in NtotMassive) \{

for ( $\mathrm{k}$ in NtotMassive) \{

linkM =

linker (MassiveChain [1], SizeOfMassive [1], MassiveChain [k], SizeOfMassive [k]);

//IF THE LINKING NUMBER BETWEEN ANY MASSIVE RINGS

//IS NON ZERO $\rightarrow>$ RETURN WARNING AND STOP COMPUTATION

if (linkM!=0) $\quad$ cout $\ll$ "WARNING LK $!=0 "<<e n d l ; \operatorname{cin} \cdot \operatorname{get}() ; \operatorname{cin} . \operatorname{get}() ;\}$

\}

\}

//OTHERWISE -- IF LK==0

//COMPUTE LINKING BETWEEN MASSIVE RINGS AND EXTENDED STRANDS

//LOOP OVER MASSIVE RINGS

for (nm in NtotMassive) \{

//START LOOP OVER ALL OTHER MASSIVE CHAINS IN SAME CUBE

for (nm1 in NtotMassive) \{

//IF NOT THE SAME MASSIVE CHAIN

if $(\mathrm{nm} 1 !=\mathrm{nm})\{$

//START LOOP OVER STRANDS WHICH MAKE THE MASSIVE RING

for ( $j$ in NumOfStrandsOfMassive[nm1]) \{ 
//COMPUTE LINKING NMBER BETWEEN RING AND EXTENDED STRAND //MADE FROM THAT STRAND

link = linker (MassiveChain $[\mathrm{nm}]$, Size0fMassive $[\mathrm{nm}]$,

ExtendedStrands [nm1] $[j], \operatorname{SizeOfExtStr}[\mathrm{nm} 1][j])$;

//REGISTER LINK BETWEEN MASSIVE AND EXTENDED STRAND ->

//REGISTER "LINK" BETWEEN THE REAL RINGS

//MAKING THE VIRTUAL MASSIVE RINGS nm AND nm1

if (link>0) PmapOfCube [ChainOfMassive [nm] ] [ChainOfMassive [nm1]] [nc] ++;

if (link>0) Pmap [ChainOfMassive [nm] ] [ChainOfMassive [nm1] ]++;

\}//CLOSE LOOP OVER STRANDS IN MASSIVE nm1

\}//CLOSE IF

\}//CLOSE LOOP OVER MASSIVES nm1

\}//CLOSE LOOP OVER MASSIVES $\mathrm{nm}$

\}//CLOSE LOOP OVER CUBES

$/ / / / / / / / / / / / / / / / / / / / / / / / / / /$

//COMPUTE THREADINGS BETWEEN

//I AND J FROM PMAP

for ( $i$ in chains)for ( $j$ in chains)

for (nc in Ncubes) if (Pmap [i] $[j][n c]>2)$

$\operatorname{Th}[i][j]+=$ PmapOfCube $[i][j][\mathrm{nc}] / 2$;

//TOTAL THREADINGS BETWEEN CHAINS

for ( $i$ in chains)for ( $j$ in chains)ThTOT $+=$ Th $[i][j]$;

$/ / / / / / / / / / / / / / / / / / / / / / / / / / / / /$

\}//CLOSE LOOP OVER TIME

return 0 ;

\}//CLOSE INT MAIN

//////////////////////////////////////////////

///////////////////////////////////////////

//FUNCTIONS

////////////////////////////////////////////

$/ / / / / / / / / / / / / / / / / / / / / / / / / / / / / / /$

$/ /$ FIND IF BEAD IS IN CUBE $/ / / /$

$/ / / / / / / / / / / / / / / / / / / / / / / / / / / / / / / /$

int where(double pos[], double LX, double LY, double LZ) \{

double eps $=0.5$;

double $1 \mathrm{Cell}=10.0+2.0 *$ eps;

double Lx = LX - eps;

double Ly = LY - eps;

double Lz = LZ - eps;

if $((\operatorname{pos}[0]<\mathrm{Lx}+1 \mathrm{Cell} \& \&$ pos $[0]>\mathrm{Lx}) \& \&((\operatorname{pos}[1]<\mathrm{Ly}+1 \mathrm{Cell} \& \&$ pos $[1]>\mathrm{Ly}) \& \&$ $(\operatorname{pos}[2]<\mathrm{Lz}+1 \mathrm{Cell} \& \& \operatorname{pos}[2]>\mathrm{Lz})))$ return 1 ; \}

//////////////////////////////////////////////////////// //USE GAUSS FORMULA TO COMPUTE LINKING NUMBER OF CLOSED CURVES// $/ / / / / / / / / / / / / / / / / / / / / / / / / / / / / / / / / / / / / / / / / / / / / / / / / / / / / / / /$ double linker(double c1[][3], int n1, double c2[][3], int n2) \{ 


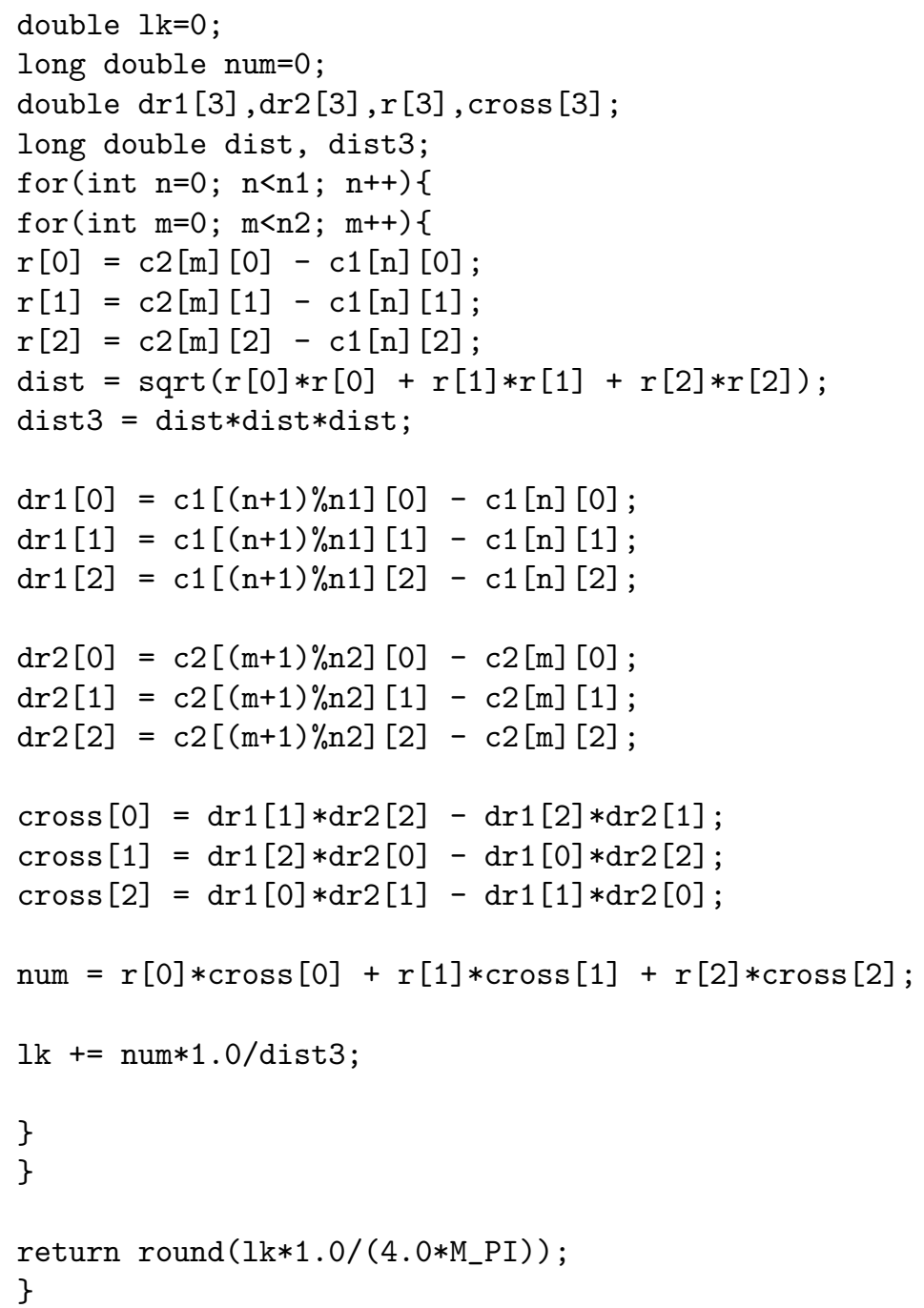




\section{Bibliography}

[Adam and Gibbs, 1965] Adam, G. and Gibbs, J. H., On the Temperature Dependence of Cooperative Relaxation Properties in Glass-Forming Liquids, J. Chem. Phys., 43(1), 139 (1965)

[Adams, 1994] Adams, C. C., The Knot Book: An Elementary Introduction to the Mathematical Theory of Knots, W H Freeman and Company (New York) (1994)

[Aichele and Baschnagel, 2001] Aichele, M. and Baschnagel, J., Glassy dynamics of simulated polymer melts: Coherent scattering and van Hove correlation functions, Eur. Phys. J. E, 5(2), 229 (2001)

[Åkerman, 1998] Åkerman, B., Effects of supercoiling in electrophoretic trapping of circular DNA in polyacrylamide gels., Biophys. J., 74(6), 3140 (1998)

[Akerman and Cole, 2002] Åkerman, B. and Cole, K., Electrophoretic capture of circular DNA in gels, Electrophoresis, pages 2549-2561 (2002)

[Alberts et al., 2014] Alberts, B., Johnson, A., Lewis, J., Morgan, D., and Raff, M., Molecular Biology of the Cell, Taylor \& Francis (2014)

[Alder and Wainwright, 1957] Alder, B. J. and Wainwright, T. E., Phase Transition for a Hard Sphere System, J. Chem. Phys., 27(5), 1208 (1957)

[Alder and Wainwright, 1959] Alder, B. J. and Wainwright, T. E., Studies in Molecular Dynamics. I. General Method, J. Chem. Phys., 31(2), 459 (1959)

[Alon and Mukamel, 1997] Alon, U. and Mukamel, D., Gel electrophoresis and diffusion of ring-shaped DNA, Phys. Rev. E, 55(2), 1783 (1997)

[Arsuaga et al., 2005] Arsuaga, J., Vazquez, M., McGuirk, P., Trigueros, S., Sumners, D. W., and Roca, J., DNA knots reveal a chiral organization of DNA in phage capsids., Proc. Natl. Acad. Sci. USA, 102(26), 9165 (2005)

[Arsuaga et al., 2002] Arsuaga, J., Vázquez, M., Trigueros, S., Sumners, D., and Roca, J., Knotting probability of DNA molecules confined in restricted volumes: DNA knotting in phage capsids., Proc. Natl. Acad. Sci. USA, 99(8), 5373 (2002)

[Avliyakulov et al., 2004] Avliyakulov, N. K., Lukes, J., and Ray, D. S., Mitochondrial Histone-Like DNA-Binding Proteins Are Essential for Normal Cell Growth and Mitochondrial Function in Crithidia fasciculata, Eukaryot. Cell, 3(2), 518 (2004)

[Baerts et al., 2013] Baerts, P., Basu, U., Maes, C., and Safaverdi, S., Frenetic origin of negative differential response, Phys. Rev. E, 88(5), 052109 (2013) 
[Baiesi et al., 2009] Baiesi, M., Maes, C., and Wynants, B., Fluctuations and Response of Nonequilibrium States, Phys. Rev. Lett., 103(1), 010602 (2009)

[Baiesi et al., 2011] Baiesi, M., Maes, C., and Wynants, B., The modified Sutherland-Einstein relation for diffusive non-equilibria, Proc. R. Soc. A, 467(2134), 2792 (2011)

[Barkema et al., 1994] Barkema, G., Marko, J., and Widom, B., Electrophoresis of charged polymers: simulation and scaling in a lattice model of reptation, Phys. Rev. E, 49(6) (1994)

[Bates and Maxwell, 2005] Bates, A. and Maxwell, A., DNA topology, Oxford University Press (2005)

[Berger et al., 1996] Berger, J., Gamblin, S., Harrison, S., and Wang, J., Structure and mechanism of DNA topoisomerase II, Nature, 379, 225 (1996)

[Bernabei et al., 2013] Bernabei, M., Bacova, P., Moreno, A. J., Narros, A., and Likos, C. N., Fluids of semiflexible ring polymers: effective potentials and clustering, Soft Matter, 9(4), 1287 (2013)

[Berthier and Biroli, 2011] Berthier, L. and Biroli, G., Theoretical perspective on the glass transition and amorphous materials, Rev. Mod. Phys., 83(2), 587 (2011)

[Biroli et al., 2008] Biroli, G., Bouchaud, J.-P., Cavagna, A., Grigera, T. S., and Verrocchio, P., Thermodynamic signature of growing amorphous order in glassforming liquids, Nature Physics, 4(10), 771 (2008)

[Bogle et al., 1994] Bogle, M. G. V., Hearst, J. E., Jones, V. F. R., and Stoilov, L., Lissajous Knots, J. Knot Theory Ramifications, 3(2), 121 (1994)

[Bohec et al., 2013] Bohec, P., Gallet, F., Maes, C., Safaverdi, S., Visco, P., and Van Wijland, F., Probing active forces via a fluctuation-dissipation relation: Application to living cells, Europhys. Lett., 102, 50005 (2013)

[Borst, 1991] Borst, P., Why kinetoplast DNA networks?, Trends Genet., 7 (1991)

[Bouchaud and Biroli, 2004] Bouchaud, J.-P. and Biroli, G., On the Adam-GibbsKirkpatrick-Thirumalai-Wolynes scenario for the viscosity increase in glasses., J. Chem. Phys., 121(15), 7347 (2004)

[Brackley et al., 2014] Brackley, C. A., Morozov, A. N., and Marenduzzo, D., Models for twistable elastic polymers in Brownian dynamics, and their implementation for LAMMPS., J. Chem. Phys., 140(13), 135103 (2014)

[Brás et al., 2014] Brás, A., Gooßen, S., and Krutyeva, M., Compact structure and non-Gaussian dynamics of ring polymer melts, Soft Matter, 10, 3649 (2014)

[Broedersz and MacKintosh, 2014] Broedersz, C. P. and MacKintosh, F. C., Modeling semiflexible polymer networks, Rev. Mod. Phys., 86(3), 995 (2014)

[Brown and Cozzarelli, 1981] Brown, P. O. and Cozzarelli, N. R., Catenation and knotting of duplex DNA by type 1 topoisomerases: a mechanistic parallel with type 2 topoisomerases., Proc. Natl. Acad. Sci. USA, 78(2), 843 (1981) 
[Calladine et al., 1991] Calladine, C. R., Collis, C. M., Drew, H. R., and Mott, M. R., A study of electrophoretic mobility of DNA in agarose and polyacrylamide gels., J. Mol. Biol., 221(3), 981 (1991)

[Calladine et al., 1997] Calladine, C. R., Drew, H., Luisi, F. B., and Travers, A. A., Understanding DNA: the molecule and how it works, Elsevier Academic Press (1997)

[Cammarota, 2009] Cammarota, C., Ph.D. thesis, La Sapienza (Roma) (2009)

[Cammarota, 2013] Cammarota, C., A general approach to systems with randomly pinned particles: Unfolding and clarifying the Random Pinning Glass Transition, Europhys. Lett., 101(5), 56001 (2013)

[Cammarota and Biroli, 2012] Cammarota, C. and Biroli, G., Ideal glass transitions by random pinning, Proc. Natl. Acad. Sci. USA, 109(23), 8850 (2012)

[Carmesin and Kremer, 1988] Carmesin, I. and Kremer, K., The bond fluctuation method: a new effective algorithm for the dynamics of polymers in all spatial dimensions, Macromolecules, 21, 2819 (1988)

[Cates and Deutsch, 1986] Cates, M. and Deutsch, J., Conjectures on the statistics of ring polymers, J. Phys. Paris, 47, 2121 (1986)

[Cavalli and Misteli, 2013] Cavalli, G. and Misteli, T., Functional implications of genome topology., Nat. Struct. Mol. Biol., 20(3), 290 (2013)

[Cebrián et al., 2014] Cebrián, J., Kadomatsu-Hermosa, M. J., Castán, A., Martínez, V., Parra, C., Fernández-Nestosa, M. J., Schaerer, C., MartínezRobles, M.-L., Hernández, P., Krimer, D. B., Stasiak, A., and Schvartzman, J. B., Electrophoretic mobility of supercoiled, catenated and knotted DNA molecules., Nucleic Acids Res., 3112(Ext 4232), 1 (2014)

[Chen et al., 1995a] Chen, J., Englund, P. T., and Cozzarelli, N. R., Changes in network topology during the replication of kinetoplast DNA., EMBO J., 14(24), 6339 (1995)

[Chen et al., 1995b] Chen, J., Rauch, C. A., White, J. H., Englund, P. T., and Cozzarelli, N. R., The topology of the kinetoplast DNA network., Cell, 80(1), $61(1995)$

[Cole and Åkerman, 2003] Cole, K. D. and Åkerman, B., The Influence Of Agarose Concentration In Gels On The Electrophoretic Trapping Of Circular Dna, Separ. Sci. Technol., 38(10), 2121 (2003)

[Cremer and Cremer, 2001] Cremer, T. and Cremer, C., Chromosome territories, nuclear architecture and gene regulation in mammalian cells., Nat. Rev. Genet., 2(4), 292 (2001)

[Daoud and Joanny, 1981] Daoud, M. and Joanny, J., Conformation of branched polymers, Journal de physique, 42(10), 1359 (1981)

[de Gennes, 1971] de Gennes, P. G., Reptation of a Polymer Chain in the Presence of Fixed Obstacles, J. Chem. Phys., 55(2), 572 (1971) 
[de Gennes, 1979] de Gennes, P. G., Scaling concepts in polymer physics, Cornell University Press (1979)

[de Gennes, 1981] de Gennes, P. G., Coherent scattering by one reptating chain, J. Phys. (Paris), 42(5), 735 (1981)

[de Souza et al., 2009] de Souza, W., Attias, M., and Rodrigues, J. C. F., Particularities of mitochondrial structure in parasitic protists (Apicomplexa and Kinetoplastida)., Int. J. Biochem. Cell. B., 41(10), 2069 (2009)

[Di Stefano et al., 2014] Di Stefano, M., Tubiana, L., Di Ventra, M., and Micheletti, C., Driving knots on DNA with $A C / D C$ electric fields: topological friction and memory effects, Soft matter, 10, 6491 (2014)

[Diao et al., 2003] Diao, Y., Dobay, A., Kusner, R. B., Millett, K., and Stasiak, A., The average crossing number of equilateral random polygons, J. Phys. A: Math. Gen., 36(46), 11561 (2003)

[Diao et al., 2012] Diao, Y., Hinson, K., Kaplan, R., Vazquez, M., and Arsuaga, J., The effects of density on the topological structure of the mitochondrial DNA from trypanosomes, J. Math. Biol., 64, 1087 (2012)

[Dignam et al., 1983] Dignam, J., Lebovitz, R., and Roeder, R., Accurate transcription initiation by RNA polymerase II in a soluble extract from isolated mammalian nuclei, Nucleic Acids Res., 1(5), 1475 (1983)

[Docampo et al., 2010] Docampo, R., Ulrich, P., and Moreno, S. N. J., Evolution of acidocalcisomes and their role in polyphosphate storage and osmoregulation in eukaryotic microbes, Phil. Trans. R. Soc. B, 365(February), 775 (2010)

[Doi and Edwards, 1988] Doi, M. and Edwards, S., The theory of polymer dynamics, Oxford University Press (1988)

[Doi et al., 2015] Doi, Y., Matsubara, K., Ohta, Y., Nakano, T., Kawaguchi, D., Takahashi, Y., Takano, A., and Matsushita, Y., Melt Rheology of Ring Polystyrenes with Ultrahigh Purity, Macromolecules, 48(9), 3140 (2015)

[Dorfman, 2010] Dorfman, K. D., DNA electrophoresis in microfabricated devices, Rev. Mod. Phys., 82(4), 2903 (2010)

[Drew and Englund, 2001] Drew, M. E. and Englund, P. T., Intramitochondrial location and dynamics of Crithidia fasciculata kinetoplast minicircle replication intermediates., J. Cell Biol., 153(4), 735 (2001)

[Duke, 1989] Duke, T., Tube model of field-inversion electrophoresis, Phys. Rev. Lett., 62(24), 2877 (1989)

[Duke et al., 1996] Duke, T., Austin, R., Cox, E., and Chan, S., Pulsed field electrophoresis in microlithographic arrays, Electrophoresis, 17, 1075 (1996)

[Duplantier et al., 1995] Duplantier, B., Jannink, G., and Sikorav, J. L., Anaphase chromatid motion: involvement of type II DNA topoisomerases, Biophys. J., 69(4), 1596 (1995) 
[Edwards, 1967] Edwards, S., Statistical mechanics with topological constraints: I, Proc. Phys. Soc., 91, 513 (1967)

[Edwards, 1968] Edwards, S., Statistical mechanics with topological constraints: II, J. Phys. A: Math. Gen., 1, 15 (1968)

[Englund, 1978] Englund, P., The replication of kinetoplast DNA networks in Crithidia fasciculata, Cell, 14(May), 157 (1978)

[Englund, 1979] Englund, P., Free minicircles of kinetoplast DNA in Crithidia fasciculata., J. Biol. Chem., pages 4895-4900 (1979)

[Fairlamb et al., 1978] Fairlamb, A. H., Weislogel, P. O., Hoeijmakers, J. H., and Borst, P., Isolation and characterization of kinetoplast DNA from bloodstream form of Trypanosoma brucei., J. Cell Biol., 76(2), 293 (1978)

[Flory, 1953] Flory, P. J., Principles of polymer chemistry, Cornell University Press (Ithaca, New York) (1953)

[Frenkel and Smit, 2001] Frenkel, D. and Smit, B., Understanding molecular simulation: from algorithms to applications, Academic Press (2001)

[Frey et al., 2015] Frey, S., Weysser, F., Meyer, H., Farago, J., Fuchs, M., and Baschnagel, J., Simulated glass-forming polymer melts: Dynamic scattering functions, chain length effects, and mode-coupling theory analysis, Eur. Phys. J. E, 38(11), 1 (2015)

[Ghosh et al., 2014] Ghosh, P. K., Hänggi, P., Marchesoni, F., and Nori, F., Giant negative mobility of Janus particles in a corrugated channel, Phys. Rev. E, 89(6), 062115 (2014)

[Gluenz et al., 2007] Gluenz, E., Shaw, M. K., and Gull, K., Structural asymmetry and discrete nucleic acid subdomains in the Trypanosoma brucei kinetoplast, Mol. Microbiol., 64(May), 1529 (2007)

[Gokhale et al., 2014] Gokhale, S., Nagamanasa, K. H., Ganapathy, R., and Sood, a. K., Growing Dynamical Facilitation on Approaching the Random Pinning Colloidal Glass Transition, Nat. Commun., 5, 1 (2014)

[Gooßen et al., 2014] Gooßen, S., Brás, A. R., Krutyeva, M., Sharp, M., Falus, P., Feoktystov, A., Gasser, U., Wischnewski, A., and Richter, D., Molecular Scale Dynamics of Large Ring Polymers, Phys. Rev. Lett., 113, 169302 (2014)

[Grosberg, 2014] Grosberg, A., Annealed lattice animal model and Flory theory for the melt of non-concatenated rings: towards the physics of crumpling, Soft Matter, 10, 560 (2014)

[Grosberg et al., 1993] Grosberg, A. Y., Rabin, Y., Havlin, S., and Neer, A., Crumpled globule model of the three-dimensional structure of DNA, Europhys. Lett., 23(5), 373 (1993)

[Guenet and Rochas, 2006] Guenet, J. M. and Rochas, C., Agarose sols and gels revisited, Macromol. Symp., 242, 65 (2006) 
[Gutin et al., 1993] Gutin, A., Grosberg, A., and Shakhnovich, E., Polymers with annealed and quenched branchings belong to different universality classes, Macromolecules, 26(5), 1293 (1993)

[Halverson et al., 2012] Halverson, J. D., Grest, G., Grosberg, A. Y., and Kremer, K., Rheology of Ring Polymer Melts: From Linear Contaminants to RingLinear Blends, Phys. Rev. Lett., 108(3), 038301 (2012)

[Halverson et al., 2013] Halverson, J. D., Kremer, K., and Grosberg, A. Y., Comparing the results of lattice and off-lattice simulations for the melt of nonconcatenated rings, J. Phys. A, 46(6), 065002 (2013)

[Halverson et al., 2011a] Halverson, J. D., Lee, W. B., Grest, G. S., Grosberg, A. Y., and Kremer, K., Molecular dynamics simulation study of nonconcatenated ring polymers in a melt. I. Statics., J. Chem. Phys., 134(20), 204904 (2011)

[Halverson et al., 2011b] Halverson, J. D., Lee, W. B., Grest, G. S., Grosberg, A. Y., and Kremer, K., Molecular dynamics simulation study of nonconcatenated ring polymers in a melt. II. Dynamics., J. Chem. Phys., 134(20), 204905 (2011)

[Halverson et al., 2014] Halverson, J. D., Smrek, J., Kremer, K., and Grosberg, A., From a melt of rings to chromosome territories: the role of topological constraints in genome folding, Rep. Prog. Phys., 77, 022601 (2014)

[Hines and Ray, 1998] Hines, J. C. and Ray, D. S., The Crithidia fasciculata KAP 1 gene encodes a highly basic protein associated with kinetoplast DNA 1, Mol. Biochem. Parasit., 94, 41 (1998)

[Hsieh and Brutlag, 1980] Hsieh, T. and Brutlag, D., ATP-dependent DNA topoisomerase from D. melanogaster reversibly catenates duplex DNA rings, Cell, $\mathbf{2 1}$, $115(1980)$

[Isaacson and Lubensky, 1980] Isaacson, J. and Lubensky, T. C., Flory exponents for generalized polymer problems, J. Physique, 41, 469 (1980)

[Iyer and Arya, 2012] Iyer, B. V. S. and Arya, G., Lattice animal model of chromosome organization, Phys. Rev. E, 86(1), 011911 (2012)

[Jensen and Englund, 2012] Jensen, R. E. and Englund, P. T., Network news: the replication of kinetoplast DNA., Annu. Rev. Microbiol., 66, 473 (2012)

[Kane and Lubensky, 2013] Kane, C. L. and Lubensky, T. C., Topological boundary modes in isostatic lattices, Nature Phys., 10(1), 39 (2013)

[Kapnistos et al., 2008] Kapnistos, M., Lang, M., Vlassopoulos, D., PyckhoutHintzen, W., Richter, D., Cho, D., Chang, T., and Rubinstein, M., Unexpected power-law stress relaxation of entangled ring polymers., Nat. Mater., 7(12), 997 (2008)

[Karmakar and Parisi, 2013] Karmakar, S. and Parisi, G., Random pinning glass model, Proc. Natl. Acad. Sci. USA, 110(8), 1 (2013)

[Karplus and Petsko, 1990] Karplus, M. and Petsko, G., Molecular dynamics simulations in biology, Nature, 347 (1990) 
[Katritch et al., 1996a] Katritch, V., Bednar, J., Michoud, D., Scharein, R., Dubochet, J., and Stasiak, A., Geometry and physics of knots, Nature, 384, 142 (1996)

[Katritch et al., 1996b] Katritch, V., Bednar, J., Michoud, D., Scharein, R., Dubochet, J., and Stasiak, A., Geometry and physics of knots, Nature, 384, 142 (1996)

[Kellenberger et al., 1986] Kellenberger, E., Carlemalm, E., Sechaud, J., Ryter, A., and Haller, G., Considerations on the condensation and the degree of compactness in non-eukaryotic dna-containing plasmas, Bacterial Chromatin ed. CO Gualerzi and CL Pon, pages 11-25 (1986)

[Kitchin et al., 1984] Kitchin, P., Klein, V., Fein, B., and Englund, P., Gapped Minicircles. A novel replication intermediate of kinetoplast DNA., J. Biol. Chem., $\mathbf{2 5 9}(24), 15532$ (1984)

[Klein, 1986] Klein, J., Dynamics of entangled linear, branched, and cyclic polymers, Macromolecules, 118(33), 105 (1986)

[Kob et al., 1997] Kob, W., Donati, C., Plimpton, S., Poole, P., and Glotzer, S., Dynamical Heterogeneities in a Supercooled Lennard-Jones Liquid, Phys. Rev. Lett., 79(15), 2827 (1997)

[Kolahi et al., 2012] Kolahi, K. S., Donjacour, A., Liu, X., Lin, W., Simbulan, R. K., Bloise, E., Maltepe, E., and Rinaudo, P., Effect of substrate stiffness on early mouse embryo development., PloS one, 7(7), e41717 (2012)

[Krasnow and Cozzarelli, 1982] Krasnow, M. and Cozzarelli, N., Catenation of DNA rings by topoisomerases, J. Biol. Chem., 257, 2687 (1982)

[Kremer and Grest, 1990] Kremer, K. and Grest, G. S., Dynamics of entangled linear polymer melts: A molecular-dynamics simulation, J. Chem. Phys., 92(8), 5057 (1990)

[Kreuzer and Cozzarelli, 1980] Kreuzer, K. and Cozzarelli, N., Formation and resolution of DNA catenanes by DNA gyrase, Cell, 20(May), 245 (1980)

[Kusner and Sullivan, 1994] Kusner, R. and Sullivan, J., Möbius energies for knots and links, surfaces and submanifolds, Geometric Topology (Proceedings of the 1993 Georgia International Topology Conference) AMS/IP Studies in Adv. Math., pages 570-604 (1994)

[Kusner and Sullivan, 1998] Kusner, R. and Sullivan, J., Möbius-Invariant Knot Energies, in eds. L. Kauffman, V. Katritch, A. Stasiak, editor, Ideal Knots, pages 315-352, World Scientific Press (1998)

[Lai et al., 2008] Lai, D.-h., Hashimi, H., Lun, Z.-r., Ayala, F. J., and Lukes, J., Adaptations of Trypanosoma brucei to gradual loss of kinetoplast DNA : Trypanosoma equiperdum and Trypanosoma evansi are petite mutants of $T$. brucei, Proc. Natl. Acad. Sci. USA, 105(6), 1999 (2008)

[Lee et al., 2015] Lee, E., Kim, S., and Jung, Y., Slowing Down of Ring Polymer Diffusion Caused by Inter-Ring Threading, Macromol. Rapid Commun., pages $\mathrm{n} / \mathrm{a}-\mathrm{n} / \mathrm{a}(2015)$ 
[Levene and Zimm, 1987] Levene, S. D. and Zimm, B. H., Separations of opencircular DNA using pulsed-field electrophoresis., Proc. Natl. Acad. Sci. USA, 84(12), 4054 (1987)

[Lieberman-Aiden et al., 2009] Lieberman-Aiden, E., van Berkum, N. L., Williams, L., Imakaev, M., Ragoczy, T., Telling, A., Amit, I., Lajoie, B. R., Sabo, P. J., Dorschner, M. O., Sandstrom, R., Bernstein, B., Bender, M. A., Groudine, M., Gnirke, A., Stamatoyannopoulos, J., Mirny, L. A., Lander, E. S., and Dekker, J., Comprehensive mapping of long-range interactions reveals folding principles of the human genome., Science, 326(5950), 289 (2009)

[Likos et al., 2014] Likos, C. N., Narros, A., Moreno, A., and Capone, B., Multi-blob coarse graining for ring polymer solutions, Soft Matter (2014)

[Linial and Shlomai, 1988] Linial, M. and Shlomai, J., Bent DNA structures associated with several origins of replication are recognized by a unique enzyme from trypanosomatids, Nucleic Acids Res., 16(14), 6477 (1988)

[Liu et al., 2005] Liu, B., Liu, Y., Motyka, S. A., Agbo, E. E. C., and Englund, P. T., Fellowship of the rings: the replication of kinetoplast DNA., Trends Parasitol., 21(8), 363 (2005)

[Liu et al., 1981] Liu, L., Perkocha, L., Calendar, R., and Wang, J. C., Knotted DNA from bacteriophage capsids, Proc. Natl. Acad. Sci. USA, 78(9), 5498 (1981)

[Liu et al., 1976] Liu, L. F., Depew, R. E., and Wang, J. C., Knotted single-stranded DNA rings: A novel topological isomer of circular single-stranded DNA formed by treatment with Escherichia coli w protein, J. Mol. Biol., 106, 439 (1976)

[Lo and Turner, 2013] Lo, W.-C. and Turner, M. S., The topological glass in ring polymers, Europhys. Lett., 102(5), 58005 (2013)

[Lubensky and Isaacson, 1979] Lubensky, T. and Isaacson, J., Statistics of lattice animals and dilute branched polymers, Physical Review A, 20(5), 2130 (1979)

[Lukes et al., 2005] Lukes, J., Hashimi, H., and Zíková, A., Unexplained complexity of the mitochondrial genome and transcriptome in kinetoplastid flagellates., Curr. Genet., 48(5), 277 (2005)

[Lukeš et al., 2002] Lukeš, J., Guilbride, D., and Votýpka, J., Kinetoplast DNA network: evolution of an improbable structure, Eukaryotic Cell, 1(4), 495 (2002)

[Lukeš et al., 2001] Lukeš, J., Hines, J., Evans, C., Avliyakulov, N. K., Prabhu, V. P., Chen, J., and Ray, D. S., Disruption of the Crithidia fasciculata KAP1 gene results in structural rearrangement of the kinetoplast disc, Mol. Biochem. Parasit., 117, 179 (2001)

[Maaloum et al., 1998] Maaloum, M., Pernodet, N., and Tinland, B., Agarose gel structure using atomic force microscopy: gel concentration and ionic strength effects, Electrophoresis, 19, 1606 (1998)

[MacKerell et al., 1998] MacKerell, A. D., Bashford, D., Bellott, M., Dunbrack, R. L., Evanseck, J. D., Field, M. J., Fischer, S., Gao, J., Guo, H., Ha, S., 
Joseph-McCarthy, D., Kuchnir, L., Kuczera, K., Lau, F. T., Mattos, C., Michnick, S., Ngo, T., Nguyen, D. T., Prodhom, B., Reiher, W. E., Roux, B., Schlenkrich, M., Smith, J. C., Stote, R., Straub, J., Watanabe, M., Wiórkiewicz-Kuczera, J., Yin, D., and Karplus, M., All-atom empirical potential for molecular modeling and dynamics studies of proteins., J. Phys. Chem. B, 102(18), 3586 (1998)

[Maffeo et al., 2010] Maffeo, C., Schöpflin, R., Brutzer, H., Stehr, R., Aksimentiev, A., Wedemann, G., and Seidel, R., DNA-DNA Interactions in Tight Supercoils Are Described by a Small Effective Charge Density, Phys. Rev. Lett., 105(15), 158101 (2010)

[Marini et al., 2015] Marini, B., Kertesz-Farkas, A., Ali, H., Lucic, B., Lisek, K., Manganaro, L., Pongor, S., Luzzati, R., Recchia, A., Mavilio, F., Giacca, M., and Lusic, M., Nuclear architecture dictates HIV-1 integration site selection, Nature, 521(7551), 227 (2015)

[Marini et al., 1982] Marini, J. C., Levene, S. D., Crothers, D. M., and Englund, P. T., Bent helical structure in kinetoplast DNA, Proc. Natl. Acad. Sci. USA, 79 (1982)

[Marko and Cocco, 2003] Marko, J. and Cocco, S., The micromechanics of DNA, Phys. World, pages 37-41 (2003)

[Matthews, 1968] Matthews, B., Solvent content of protein crystals, J. Mol. Biol., 33(August 1967), 491 (1968)

[Maxwell and Gellert, 1984] Maxwell, A. and Gellert, M., The DNA dependence of the ATPase activity of DNA gyrase., J. Biol. Chem., 259(23), 14472 (1984)

[Maxwell, 1864] Maxwell, J. C., L. on the calculation of the equilibrium and stiffness of frames, Phil. Mag., 27, 294 (1864)

[McCammon et al., 1977] McCammon, J., Gelin, B., and Karplus, M., Dynamics of folded proteins, Nature, 267 (1977)

[Melendy et al., 1988] Melendy, T., Sheline, C., and Ray, D. S., Localization of a type II DNA topoisomerase to two sites at the periphery of the kinetoplast DNA of Crithidia fasciculata., Cell, 55, 1083 (1988)

[Mézard and Parisi, 2001] Mézard, M. and Parisi, G., The Bethe lattice spin glass revisited, Eur. Phys. J. B, 20, 217 (2001)

[Micheletti et al., 2015] Micheletti, C., Di Stefano, M., and Orland, H., Absence of knots in known RNA structures., Proc. Natl. Acad. Sci. USA, 112(7), 2052 (2015)

[Micheletti et al., 2011] Micheletti, C., Marenduzzo, D., and Orlandini, E., Polymers with spatial or topological constraints: Theoretical and computational results, Phys. Rep., 504(1), 1 (2011)

[Michieletto et al., 2014] Michieletto, D., Marenduzzo, D., and Turner, M. S., Topology Regulation during Replication of the Kinetoplast DNA, arXiv preprint arXiv:1408.4237 (2014) 
[Mickel et al., 1977] Mickel, S., Arena, V., and Bauer, W., Physical properties and gel electrophoresis behavior of R12-derived plasmid DNAs, Nucleic Acids Res., 4(5), 1465 (1977)

[Milner and Newhall, 2010] Milner, S. and Newhall, J., Stress Relaxation in Entangled Melts of Unlinked Ring Polymers, Phys. Rev. Lett., 105(20), 208302 (2010)

[Mirny, 2011] Mirny, L. A., The fractal globule as a model of chromatin architecture in the cell., Chromosome Res., 19(1), 37 (2011)

[Mogilner and Rubinstein, 2005] Mogilner, A. and Rubinstein, B., The physics of filopodial protrusion., Biophysical J., 89(2), 782 (2005)

[Mohan and Doyle, 2007a] Mohan, A. and Doyle, P., Effect of disorder on DNA electrophoresis in a microfluidic array of obstacles, Phys. Rev. E, 76(4), 040903 (2007)

[Mohan and Doyle, 2007b] Mohan, A. and Doyle, P. S., Stochastic Modeling and Simulation of DNA Electrophoretic Separation in a Microfluidic Obstacle Array, Macromolecules, 40(24), 8794 (2007)

[Morris et al., 2001] Morris, J. C., Drew, M. E., Klingbeil, M. M., Motyka, S. a., Saxowsky, T. T., Wang, Z., and Englund, P. T., Replication of kinetoplast $D N A$ : an update for the new millennium., International journal for parasitology, 31(5-6), 453 (2001)

[Muller et al., 2000] Muller, M., Wittmer, J., and Cates, M., Topological effects in ring polymers. II. Influence Of persistence length, Phys. Rev. E, 61(4), 4078 (2000)

[Müller et al., 1996] Müller, M., Wittmer, J. P., and Cates, M. E., Topological effects in ring polymers: A computer simulation study., Phys. Rev. E, 53(5), 5063 (1996)

[Nagamanasa et al., 2015] Nagamanasa, K. H., Gokhale, S., Sood, a. K., and Ganapathy, R., Direct measurements of growing amorphous order and nonmonotonic dynamic correlations in a colloidal glass-former, Nature Physics, 11(May), 403 (2015)

[Obukhov and Rubinstein, 1994] Obukhov, S. and Rubinstein, M., Dynamics of a ring polymer in a gel, Phys. Rev. Lett., 73(9), 1263 (1994)

[Ogbadoyi et al., 2003] Ogbadoyi, E., Robinson, D., and Gull, K., A high-order trans-membrane structural linkage is responsible for mitochondrial genome positioning and segregation by flagellar basal bodies in trypanosomes, Mol. Biol. Cell, 14(May), 1769 (2003)

[Olavarrieta et al., 2002] Olavarrieta, L., Martínez-Robles, M. L., Sogo, J. M., Stasiak, A., Hernández, P., Krimer, D. B., and Schvartzman, J. B., Supercoiling, knotting and replication fork reversal in partially replicated plasmids., Nucleic Acids Res., 30(3), 656 (2002)

[Orlandini et al., 2000] Orlandini, E., Tesi, M. C., and Whittington, S. G., Polymer entanglement in melts, J. Phys. A, 33, 181 (2000) 
[Orlandini et al., 1994] Orlandini, E., Tesi, M. C., Whittington, S. G., Sumners, D. W., and Rensburg, E. J. J. V., The writhe of a self-avoiding walk, J. Phys. A: Math. Gen., 27, L333 (1994)

[Orlandini and Whittington, 2004] Orlandini, E. and Whittington, S. G., Entangled polymers in condensed phases., J. Chem. Phys., 121(23), 12094 (2004)

[Orlandini and Whittington, 2007] Orlandini, E. and Whittington, S. G., Statistical topology of closed curves: Some applications in polymer physics, Rev. Mod. Phys., 79(2), 611 (2007)

[Ozawa et al., 2015] Ozawa, M., Kob, W., Ikeda, A., and Miyazaki, K., Equilibrium phase diagram of a randomly pinned glass-former, Proc. Natl. Acad. Sci. USA, $\mathbf{1 1 2}(22), 6914$ (2015)

[Palmer et al., 1984] Palmer, R. G., Stein, D. L., Abrahams, E., and Anderson, P. W., Models of hierarchically constrained dynamics for glassy relaxation, Phys. Rev. Lett., 53(10), 958 (1984)

[Parisi and Sourlas, 1981] Parisi, G. and Sourlas, N., Critical behavior of branched polymers and the Lee-Yang edge singularity, Phys. Rev. Lett., 46(14), 871 (1981)

[Pasquino et al., 2013] Pasquino, R., Vasilakopoulos, T., Jeong, C., Lee, H., Rogers, S., Sakellariou, G., Allgaier, J., Takano, A., Bras, A., Chang, T., Goossen, S., Pyckhout-Hintzen, W., Wischnewski, A., Hadjichristidis, N., Richter, D., Rubinstein, M., and Vlassopoulos, D., Viscosity of Ring Polymer Melts, ACS Macro Lett., 2, 874 (2013)

[Pérez-Morga and Englund, 1993a] Pérez-Morga, D. and Englund, P., The structure of replicating kinetoplast DNA networks., J. Cell. Biol., 123(5) (1993)

[Pérez-Morga and Englund, 1993b] Pérez-Morga, D. L. and Englund, P. T., The attachment of minicircles to kinetoplast DNA networks during replication., Cell, 74(4), 703 (1993)

[Pernodet et al., 1997] Pernodet, N., Maaloum, M., and Tinland, B., Pore size of agarose gels by atomic force microscopy, Electrophoresis, 18, 55 (1997)

[Pierański et al., 2001] Pierański, P., Przybył, S., and Stasiak, A., Tight open knots, Europhys. J. E, 6, 123 (2001)

[Piili et al., 2013] Piili, J., Marenduzzo, D., Kaski, K., and Linna, R. P., Sedimentation of knotted polymers, Phys. Rev. E, 87(1), 012728 (2013)

[Rahman, 1964] Rahman, A., Correlations in the motion of atoms in liquid argon, Phys. Rev. A, 136(2), 405 (1964)

[Rahong et al., 2014] Rahong, S., Yasui, T., Yanagida, T., Nagashima, K., Kanai, M., Klamchuen, A., Meng, G., He, Y., Zhuge, F., Kaji, N., Kawai, T., and Baba, Y., Ultrafast and wide range analysis of DNA molecules using rigid network structure of solid nanowires., Sci. Rep., 4, 5252 (2014)

[Raphael et al., 1997] Raphael, E., Gay, C., and de Gennes, P. G., Progressive construction of an Olympic gel, J. Stat. Phys., 89, 111 (1997) 
[Rauch et al., 1993] Rauch, C. A., Pérez-Morga, D., Cozzarelli, N. R., and Englund, P. T., The absence of supercoiling in kinetoplast DNA minicircles., EMBO J., 12(2), 403 (1993)

[Renger and Wolstenholme, 1971] Renger, H. and Wolstenholme, D., Kinetoplast and other satellite DNAs of kinetoplastic and dyskinetoplastic strains of Trypanosoma, J. Cell. Biol., 50, 533 (1971)

[Renger and Wolstenholme, 1972] Renger, H. and Wolstenholme, D., The form and structure of kinetoplast DNA of Crithidia, J. Cell. Biol., 5, 346 (1972)

[Roberts and Murray, 1976] Roberts, R. J. and Murray, K., Restriction endonucleases, Crit. Rev. Biochem. Mol. Biol., 4(2), 123 (1976)

[Rosa and Everaers, 2008] Rosa, A. and Everaers, R., Structure and dynamics of interphase chromosomes, PLoS Comput. Biol., 4(8), 1 (2008)

[Rosa and Everaers, 2014] Rosa, A. and Everaers, R., Ring polymers in the melt state: The physics of crumpling, Phys. Rev. Lett., 112, 118302 (2014)

[Ross, 1964] Ross, P., Electrophoresis of DNA. I. On a relationship between electrophoresis and donnan equilibrium experiments on DNA, Biopolymers, 2, 9 (1964)

[Rubinstein, 1986] Rubinstein, M., Dynamics of ring polymers in the presence of fixed obstacles, Phys. Rev. Lett., 57(24), 3023 (1986)

[Rubinstein, 1987] Rubinstein, M., Discretized model of entangled-polymer dynamics, Phys. Rev. Lett., 59(17), 1946 (1987)

[Rubinstein and Colby, 2003] Rubinstein, M. and Colby, H. R., Polymer Physics, Oxford University Press (2003)

[Rybenkov et al., 1993] Rybenkov, V., Cozzarelli, N., and Vologodskii, A., Probability of DNA knotting and the effective diameter of the DNA double helix, Proc. Natl. Acad. Sci. USA, 90(June), 5307 (1993)

[Rybenkov et al., 1997] Rybenkov, V. V., Vologodskii, A. V., and Cozzarelli, N. R., The effect of ionic conditions on the conformations of supercoiled DNA. II. Equilibrium catenation., J. Mol. Biol., 267(2), 312 (1997)

[Schwartz and Quake, 2009] Schwartz, J. J. and Quake, S. R., Single molecule measurement of the 'speed limit' of DNA polymerase, Proc. Natl. Acad. Sci. USA, 107(3), 1254 (2009)

[Shapiro and Englund, 1995] Shapiro, T. and Englund, P., The structure and replication of kinetoplast DNA, Annu. Rev. Microbiol., 49, 117 (1995)

[Shlomai, 1994] Shlomai, J., The assembly of kinetoplast DNA., Parasitology today, 10(9), 341 (1994)

[Shlomai and Linial, 1986] Shlomai, J. and Linial, M., A Nicking Enzyme from Trypanosomatids Which Specifically Affects the Topological Linking of Duplex DNA Circles, J. Biol. Chem., 261(34), 16219 (1986) 
[Shlomai and Zadok, 1983] Shlomai, J. and Zadok, A., Reversible decatenation of kinetoplast DNA by a DNA topoisomerase from trypanosomatids, Nucleic Acids Res., 11(12), 4019 (1983)

[Silver et al., 1986] Silver, L. E., Torri, a. F., and Hajduk, S. L., Organized packaging of kinetoplast DNA networks., Cell, 47(4), 537 (1986)

[Simpson and Da Silva, 1971] Simpson, L. and Da Silva, A., Isolation and characterization of kinetoplast DNA from Leishmania tarentolae, J. Mol. Biol., 56, $443(1971)$

[Simpson, 1967] Simpson, L. P., Morphogenesis and the function of the kinetoplast in "Leishmania", Atlas de Symposia sobre a Biota Amazonica (Pathologia), 6, 231 (1967)

[Smrek and Grosberg, 2015] Smrek, J. and Grosberg, A. Y., Understanding the dynamics of rings in the melt in terms of annealed tree model, J. Phys.: Condens. Matter, 27, 064117 (2015)

[Soteros and Whittington, 1988] Soteros, C. and Whittington, S. G., Critical exponents for lattice animals with fixed cyclomatic index, J. Phys. A, 21, 2187 (1988)

[Stasiak et al., 1996] Stasiak, A., Katritch, V., Bednar, J., Michoud, D., and Dubochet, J., Electrophoretic mobility of DNA knots, Nature, 384, 122 (1996)

[Stellwagen, 2009] Stellwagen, N. C., Electrophoresis of DNA in agarose gels, polyacrylamide gels and in free solution, Electrophoresis, 30(1), 1 (2009)

[Stellwagen and Stellwagen, 2009] Stellwagen, N. C. and Stellwagen, E., Effect of the matrix on DNA electrophoretic mobility., J. Chromatogr., 1216(10), 1917 (2009)

[Swope et al., 1982] Swope, W. C., Andersen, H., Berens, P., and Wilson, K., A computer simulation method for the calculation of equilibrium constants for the formation of physical clusters of molecules: Application to small water clusters, J. Chem. Phys., 76(1), 637 (1982)

[Taniguchi et al., 2010] Taniguchi, Y., Choi, P. J., Li, G.-W., Chen, H., Babu, M., Hearn, J., Emili, A., and Xie, X. S., Quantifying E. coli proteome and transcriptome with single-molecule sensitivity in single cells., Science, 329(5991), $533(2010)$

[Tead and Kramer, 1992] Tead, S. and Kramer, E., Polymer topology and diffusion: A comparison of diffusion in linear and cyclic macromolecules, Macromolecules, 25, 3942 (1992)

[Thomas and Summers, 2009] Thomas, C. M. and Summers, D., Bacterial Plasmids, in Encyclopedia Life Sciences, John Wiley \& Sons, Inc. (2009)

[Trigueros et al., 2001] Trigueros, S., Arsuaga, J., Vazquez, M. E., Sumners, D., and Roca, J., Novel display of knotted DNA molecules by two-dimensional gel electrophoresis., Nucleic Acids Res., 29(13), E67 (2001) 
[Trigueros and Roca, 2007] Trigueros, S. and Roca, J., Production of highly knotted DNA by means of cosmid circularization inside phage capsids., BMC Biotechnol, 7, 94 (2007)

[Turmel et al., 1990] Turmel, C., Brassard, E., Slater, G. W., and Noolandi, J., Molecular detrapping and band narrowing with high frequency modulation of pulsed field electrophoresis., Nucleic Acids Res., 18(3), 569 (1990)

[Vettorel et al., 2009] Vettorel, T., Grosberg, A. Y., and Kremer, K., Statistics of polymer rings in the melt: a numerical simulation study., Phys. Biol., 6(2), 025013 (2009)

[Viovy, 2000] Viovy, J., Electrophoresis of DNA and other polyelectrolytes: Physical mechanisms, Rev. Mod. Phys., 72(3), 813 (2000)

[Viovy and Duke, 1993] Viovy, J. and Duke, T., DNA electrophoresis in polymer solutions: Ogston sieving, reptation and constraint release, Electrophoresis, 14, $322(1993)$

[Viovy et al., 1992] Viovy, J., Miomandre, F., and Miquel, M., Irreversible trapping of DNA during crossed field gel electrophoresis, Electrophoresis, 13, 1 (1992)

[Volkmuth and Austin, 1992] Volkmuth, W. and Austin, R., DNA electrophoresis in microlithographic arrays, Nature, 358, 600 (1992)

[Wang and Englund, 2001] Wang, Z. and Englund, P., RNA interference of a trypanosome topoisomerase II causes progressive loss of mitochondrial DNA, EMBO J., 20(17) (2001)

[Wang et al., 2000] Wang, Z., Morris, J. C., Drew, M. E., and Englund, P. T., Inhibition of Trypanosoma brucei gene expression by RNA interference using an integratable vector with opposing Tr7 promoters., J. Biol. Chem., 275(51), $40174(2000)$

[Weber et al., 2013] Weber, C., Carlen, M., Dietler, G., Rawdon, E. J., and Stasiak, A., Sedimentation of macroscopic rigid knots and its relation to gel electrophoretic mobility of DNA knots., Sci. Rep., 3, 1091 (2013)

[Weber et al., 2006a] Weber, C., De Los Rios, P., Dietler, G., and Stasiak, A., Simulations of electrophoretic collisions of DNA knots with gel obstacles, J. Phys.: Condens. Matter, 18(14), S161 (2006)

[Weber et al., 2006b] Weber, C., Stasiak, a., De Los Rios, P., and Dietler, G., Numerical simulation of gel electrophoresis of DNA knots in weak and strong electric fields., Biophys. J., 90(9), 3100 (2006)

[Weeks et al., 1971] Weeks, J., Chandler, D., and Andersen, H., Role of repulsive forces in determining the equilibrium structure of simple liquids, J. Chem. Phys., 54(12), 5237 (1971)

[White et al., 1987] White, J. H., Millett, K. C., and Cozzarelli, N. R., Description of the topological entanglement of DNA catenanes and knots by a powerful method involving strand passage and recombination., J. Mol. Biol., 197(3), $585(1987)$ 
[Whittington and Soteros, 1990] Whittington, S. G. and Soteros, C., Lattice animals: rigorous results and wild guesses, Disorder in Physical Systems (1990)

[Whytock and Finch, 1991] Whytock, S. and Finch, J., The substructure of agarose gels as prepared for electrophoresis, Biopolymers, 31(9), 1025 (1991)

[Wickiser et al., 2005] Wickiser, J. K., Winkler, W. C., Breaker, R. R., and Crothers, D. M., The speed of RNA transcription and metabolite binding kinetics operate an FMN riboswitch., Mol. Cell, 18(1), 49 (2005)

[Xu et al., 1996] Xu, C. W., Hines, J. C., Engel, M. L., Russell, D. G., and Ray, D. S., Nucleus-encoded histone H1-like proteins are associated with kinetoplast DNA in the trypanosomatid Crithidia fasciculata., Mol. Cell. Biol., 16(2), 564 (1996)

[Xu and Ray, 1993] Xu, C. W. and Ray, D. S., Isolation of proteins associated with kinetoplast DNA networks in vivo., Proc. Natl. Acad. Sci. USA, 90(March), 1786 (1993)

[Young and Morales, 1987] Young, D. and Morales, A., Isolations of Leishmania braziliensis (Kinetoplastida: Trypanosomatidae) from cryopreserved colombian sand flies (Diptera: Psychodidae), J. Med. Entomol., 24, 587 (1987)

[Zhang et al., 2012] Zhang, Y., McCord, R. P., Ho, Y.-J., Lajoie, B. R., Hildebrand, D. G., Simon, A. C., Becker, M. S., Alt, F. W., and Dekker, J., Spatial organization of the mouse genome and its role in recurrent chromosomal translocations., Cell, 148(5), 908 (2012)

[Zia et al., 2002] Zia, R. K. P., Praestgaard, E. L., and Mouritsen, O. G., Getting more from pushing less: Negative specific heat and conductivity in nonequilibrium steady states, Am. J. Phys., 70(4), 384 (2002) 